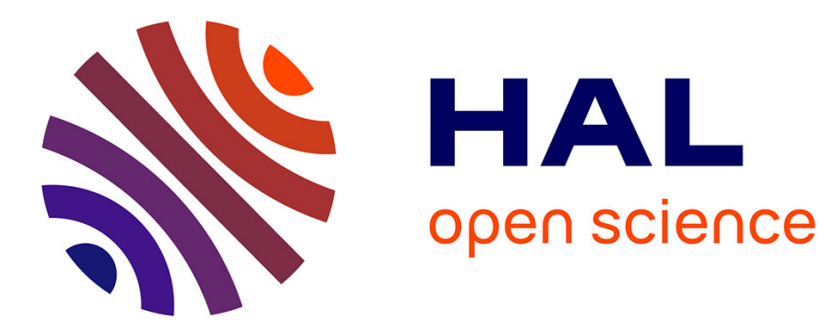

\title{
Batch replenishment planning under capacity reservation contract
}

\author{
Mlouka Farhat
}

\section{To cite this version:}

Mlouka Farhat. Batch replenishment planning under capacity reservation contract. Operations Research [cs.RO]. Université de Lorraine; École nationale d'ingénieurs de Tunis (Tunisie), 2019. English. NNT : 2019LORR0041 . tel-02347798

\section{HAL Id: tel-02347798 https://hal.univ-lorraine.fr/tel-02347798}

Submitted on 19 Jan 2021

HAL is a multi-disciplinary open access archive for the deposit and dissemination of scientific research documents, whether they are published or not. The documents may come from teaching and research institutions in France or abroad, or from public or private research centers.
L'archive ouverte pluridisciplinaire HAL, est destinée au dépôt et à la diffusion de documents scientifiques de niveau recherche, publiés ou non, émanant des établissements d'enseignement et de recherche français ou étrangers, des laboratoires publics ou privés. 


\section{(1) UNIVERSITÉ}

\section{AVERTISSEMENT}

Ce document est le fruit d'un long travail approuvé par le jury de soutenance et mis à disposition de l'ensemble de la communauté universitaire élargie.

II est soumis à la propriété intellectuelle de l'auteur. Ceci implique une obligation de citation et de référencement lors de l'utilisation de ce document.

D'autre part, toute contrefaçon, plagiat, reproduction illicite encourt une poursuite pénale.

Contact : ddoc-theses-contact@univ-lorraine.fr

\section{LIENS}

Code de la Propriété Intellectuelle. articles L 122.4

Code de la Propriété Intellectuelle. articles L 335.2- L 335.10

http://www.cfcopies.com/V2/leg/leg droi.php

http://www.culture.gouv.fr/culture/infos-pratiques/droits/protection.htm 


\section{Batch replenishment planning under capacity reservation contract}

\section{THÈSE EN COTUTELLE}

présentée et soutenue publiquement le 8 février 2019

pour l'obtention du

\section{Doctorat de l'Université de Lorraine et de l'École Nationale d'Ingénieurs de Tunis}

(Automatique, Traitement de signal et des images, Génie informatique)

(Génie industriel)

par

Mlouka Farhat

Composition du jury

Rapporteurs :

Examinateurs :

Directrices de thèse:

Mme Nathatlie SAUER, Université de Lorraine

Mme Atidel B. HADJ-ALOUANE, Ecole Nationale d'ingénieurs de Tunis

Co-Directrice de thèse: Mme Ayse AKBALIK, Université de Lorraine M. Naceur AZAIEZ, Tunis Business School

Mme Najoua DRIDI, Ecole Nationale d'ingénieurs de Tunis M. Stéphane DAUZERE-PERES, Ecole des Mines de Saint-Etienne

M. Wilco VAN DEN HEUVEL, Erasmus School of Economics Rotterdam
Mme Safia KEDAD-SIDHOUM, Conservatoire National des Arts et Métiers Paris

Laboratoire de Génie Informatique, de Production et de Maintenance EA 3096 Unité de recherche d'Optimisation et Analyse des Systèmes Industriels et de Services OASIS-UR13ES42 
Mis en page avec la classe thesul. 
This PhD research Thesis is dedicated to my: Mother, Fedia SOUIHLI; Father, Bechir FARHAT; Husband, Younes MOUMAN; Daughter, Khadija MOUMAN; and Sister Marwa FARHAT for their support, encouragement, sacrifices and being there for me throughout the entire doctorate program. May God bless and keep you always with joy, peace and happiness. 


\section{Contents}

General Introduction

Chapter 1

Literature review and problem positioning

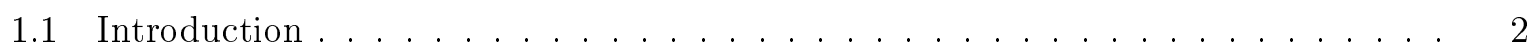

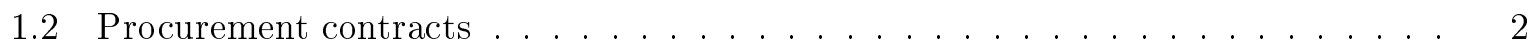

1.2 .1 Definition of procurement contracts . . . . . . . . . . . . . . 3

1.2 .2 Contracting motivations $\ldots \ldots \ldots \ldots \ldots$

1.2 .3 Research papers on procurement contracts . . . . . . . . . . . . 4

1.3 Capacity reservation contract $\ldots \ldots \ldots \ldots \ldots \ldots$

1.3.1 Description and benefits of the capacity reservation contract. . . . . . . 5

$1.3 .2 \quad$ Research papers on capacity reservation contracts . . . . . . . . . . . 6

1.4 Buyback contract . . . . . . . . . . . . . . . . . 7

$1.4 .1 \quad$ Buyback contract applications . . . . . . . . . . . . . . 7

1.4 .2 Description of the buyback contract $\ldots \ldots \ldots \ldots$. . . . . . 8

$1.4 .3 \quad$ Literature review on the buyback contract $\ldots \ldots \ldots \ldots$. . . . . . 8

1.5 Overview of lot sizing problems $\ldots \ldots \ldots \ldots \ldots \ldots \ldots$

1.5.1 Modeling the basic problem . . . . . . . . . . . . . . . . 10

1.5.2 Characteristics of the lot sizing problem . . . . . . . . . . . . . 11

$1.5 .3 \quad$ Surveys of lot sizing problems $\ldots \ldots \ldots \ldots \ldots$

1.5 .4 Resolution methods $\ldots \ldots \ldots \ldots \ldots$. . . . . . . . . . . . 14

1.6 Relevant references related to our study . . . . . . . . . . . . . . . . . 14

1.6 .1 Lot sizing problem with stepwise cost function . . . . . . . . . . . . . 15

1.6 .2 Lot sizing problem with capacity reservation contract. . . . . . . . . . . . 19

1.6 .3 Lot sizing problem with lost sales . . . . . . . . . . . . . . . . . 23

1.6 .4 Lot sizing problem with disposals . . . . . . . . . . . . . . . . . 24

$1.7 \quad$ Our contributions . . . . . . . . . . . . . . . . . . . . . . 26

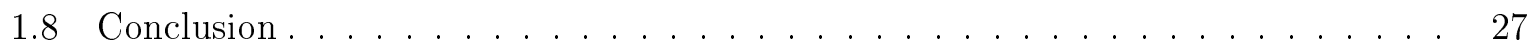




\section{Chapter 2}

Lot sizing problem with batch ordering under periodic buyback contract (LSP$\mathbf{B R}_{1}$ with $w \geq 1$ ) and lost sales

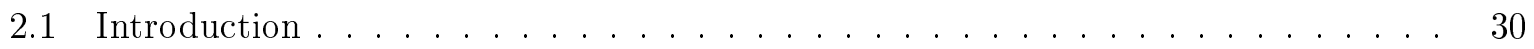

2.2 Description of the LSP-BR 1 with $w \geq 1$, hypotheses and mathematical formulation in MILP . . . . . . . . . . . . . . . . . . . . . . 30

2.3 LSP-BR $_{1}$ without lost sales $\ldots \ldots \ldots \ldots \ldots \ldots \ldots \ldots \ldots$

$2.3 .1 \quad$ Single Period problem-BR ${ }_{1}$ with only full batches $\left(\mathrm{SP}_{-} \mathrm{B}_{O F B} \mathrm{R}_{1}\right) \quad \ldots \quad \ldots 34$

$2.3 .2 \quad$ LSP-BR $_{1}$ with only full batches and $w>1\left(\right.$ LSP-B $_{O F B} \mathrm{R}_{1}$ with $\left.w>1\right)$. . 35

$2.3 .3 \quad{\mathrm{SP}-\mathrm{BR}_{1} \text { with FTL cost structure }\left(\mathrm{SP}_{-} \mathrm{B}_{F T L} \mathrm{R}_{1}\right) \ldots \ldots \ldots \ldots}^{2} \ldots \ldots \ldots$

$2.3 .4 \quad$ LSP-BR $_{1}$ with FTL cost structure and $w>1\left(\right.$ LSP-B $_{F T L} \mathrm{R}_{1}$ with $\left.w>1\right)$. 38

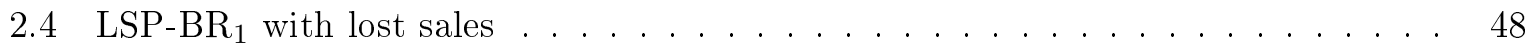

$2.4 .1 \quad$ SP-BR $_{1}$ with lost sales and only full batches $\left(\mathrm{SP}_{\left.-\mathrm{B}_{L S, O F B} \mathrm{R}_{1}\right)} \ldots \ldots . . \quad 49\right.$

$2.4 .2 \quad$ LSP-BR $_{1}$ with lost sales, only full batches and $w>1\left(\right.$ LSP-B $_{L S, O F B} \mathrm{R}_{1}$

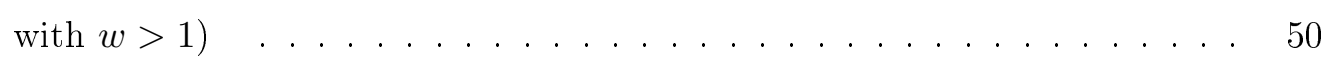

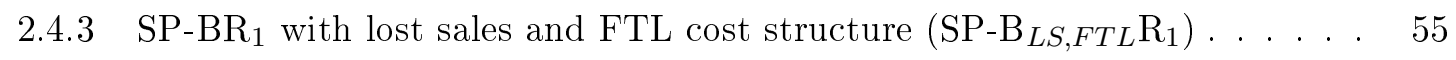

2.4.4 $\quad$ LSP-BR $_{1}$ with lost sales, FTL cost structure and $w>1\left(\right.$ LSP-B $_{L S, F T L} \mathrm{R}_{1}$

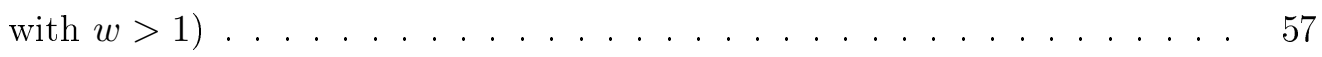

2.5 Computational experiments $\ldots \ldots \ldots \ldots \ldots \ldots \ldots \ldots$

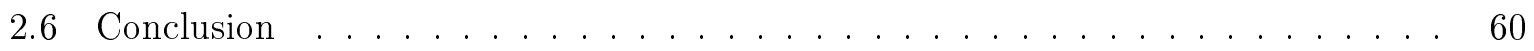

\section{Chapter 3}

Full batch replenishment with buyback and disposal options

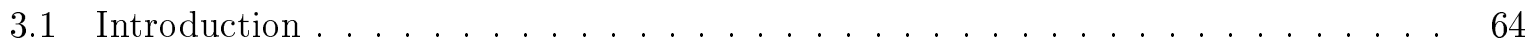

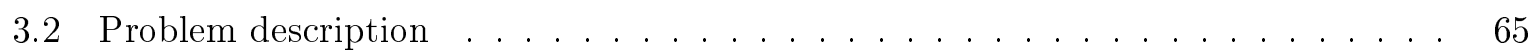

$3.3 \quad$ LSP with batch ordering, disposal and fixed return periods (LSP-BD-R ${ }_{1}$. . . . 67

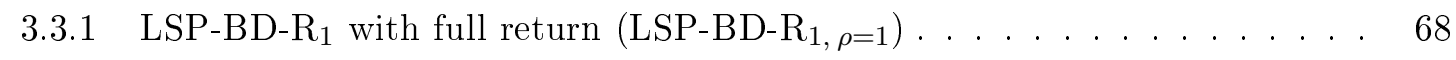

$3.3 .2 \quad$ LSP-BD-R $_{1}$ with partial return policy (LSP-BD-R $\left.1, \rho<1\right) \ldots \ldots$. . . . . 73

3.4 LSP-BD-R with time limit for returns (LSP-BD-R $\left.{ }_{2}\right) \ldots \ldots \ldots \ldots$

$3.4 .1 \quad$ LSP-BD-R $_{2}$ with full return policy $\left(\right.$ LSP-BD-R $\left.{ }_{2, \rho=1}\right) \ldots \ldots \ldots$. . . . . 79

$3.4 .2 \quad$ LSP-BD-R ${ }_{2}$ with partial return policy (LSP-BD-R $\left.\mathrm{L}_{2, \rho<1}\right) \ldots \ldots$. . . . . 83

$3.5 \quad$ LSP-BD-R with return possibility only in procurement periods (LSP-BD-R ${ }_{3}$. . $\quad 90$

$3.5 .1 \quad$ LSP-BD-R $_{3}$ with full return policy (LSP-BD-R $\left.{ }_{3, \rho=1}\right) \ldots \ldots \ldots$. . . . . 90

$3.5 .2 \quad$ LSP-BD-R $_{3}$ with partial return policy $\left(\right.$ LSP-BD-R $\left.\mathrm{R}_{3, \rho<1}\right) \ldots \ldots$. . . . . 92

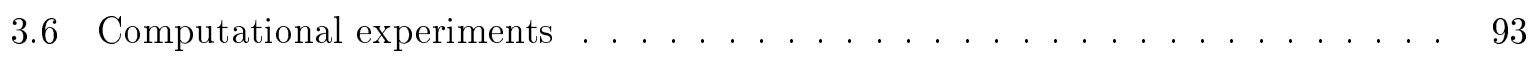

3.7 Conclusion . . . . . . . . . . . . . . . . . . . . . . . 94 


\section{Chapter 4}

Modeling lot sizing problems with batch ordering (LSP-B) under different ca-

pacity reservation contracts $(\mathrm{CRC})$

$4.1 \quad$ Introduction $\ldots \ldots \ldots \ldots \ldots \ldots \ldots$

4.2 Modeling the LSP-B-CRC $\ldots \ldots \ldots \ldots \ldots$

$4.2 .1 \quad$ LSP-B-CRC with fixed reserved capacity . . . . . . . . . . . . . . . . . . . 101

4.2.2 $\quad$ LSP-B-CRC with stationary reserved capacity being a decision variable . 101

$4.2 .3 \quad$ LSP-B-CRC with time-dependent reserved capacity being decision variable 103

4.3 LSP-B with several forms of CRC . . . . . . . . . . . . . . . . . . . . . . . 104

$4.3 .1 \quad$ LSP-B with deductible reservation contract . . . . . . . . . . . . . . . 104

4.3 .2 LSP-B with take-or-pay contract . . . . . . . . . . . . . . . . . . 105

$4.3 .3 \quad$ LSP-B with pay-to-delay contract . . . . . . . . . . . . . . . . . . . . . 106

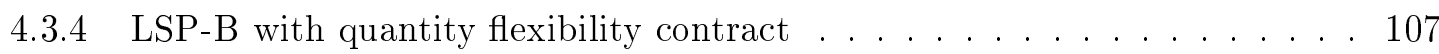

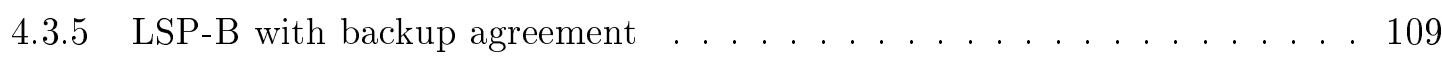

$4.3 .6 \quad$ LSP-B with minimum commitment contract . . . . . . . . . . . . . . . . . 110

4.3 .7 LSP-B with revenue-sharing contract . . . . . . . . . . . . . . . . . . 113

4.4 Conclusion . . . . . . . . . . . . . . . . . . . . . . . . . . . . . . . . 113

Conclusion and perspectives

French abstract of the dissertation

Appendix

Bibliography 


\section{List of Figures}

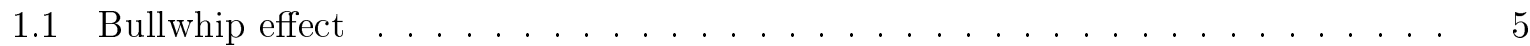

1.2 Some examples of stepwise concave functions $\ldots \ldots \ldots \ldots \ldots \ldots$

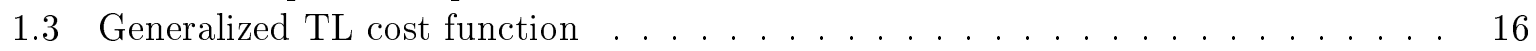

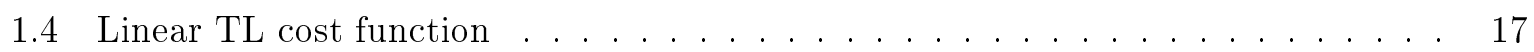

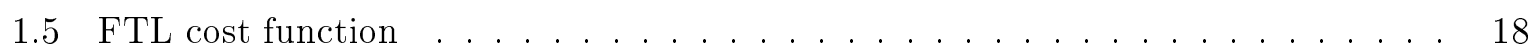

1.6 Total procurement cost function $\ldots \ldots \ldots \ldots \ldots \ldots$

1.7 Capacity reservation cost structure $\ldots \ldots \ldots \ldots \ldots \ldots$

1.8 Capacity reservation contract cost function $\ldots \ldots \ldots \ldots \ldots \ldots$

1.9 Optimal solution of an example of LSP-LS $\ldots \ldots \ldots \ldots \ldots$. . . . . . . . . . . . . . . . . . . . . . . . .

1.10 The buyback contract contributions in this study $\ldots \ldots \ldots \ldots$

$2.1 \quad$ The $N$ intervals of our problem (illustration for $w=5) \ldots \ldots \ldots$. . . . . . 34

$2.2 \quad$ Recurrence relation of $\mathrm{LSP}-\mathrm{B}_{O F B} \mathrm{R}_{1}$ with $w>1 \ldots \ldots \ldots \ldots$. . . . . . . . 37

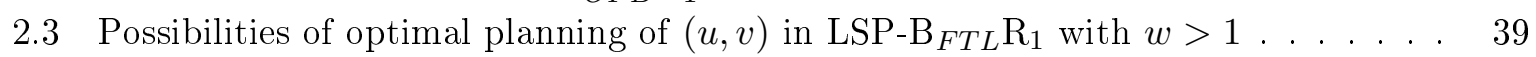

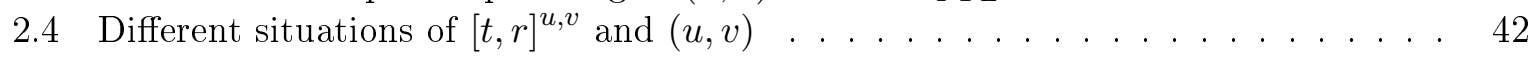

$2.5 \quad$ Possibilities of optimal planning of $(u, v)$ in LSP-B $\mathrm{B}_{L S, O F B} \mathrm{R}_{1}$ with $w>1 \ldots$. . . 53

$3.1 \quad N$ independent subproblems of the general LSP-BD-R $\ldots \ldots \ldots \ldots \ldots$. . . . . 67

3.2 How to compute the optimal $\operatorname{costs} C^{\rho}(v, \Delta) \ldots \ldots \ldots \ldots \ldots \ldots$

$3.3 \quad$ A subplan $(u, v)$ having one return in period $t<t_{\max } \ldots \ldots \ldots \ldots \ldots$

$3.4 \quad$ A subplan $(u, v)$ having one return in period $t>t_{\max } \ldots \ldots \ldots \ldots \ldots$

$3.5 \quad$ A subplan $(u, v)$ having one return in period $t_{\max _{1}}$ in policy $\pi^{(1)} \ldots \ldots$. . . 82

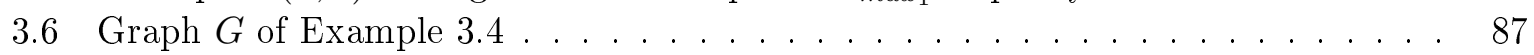

3.7 Different cases studied in Chapter $3\left(^{*}\right.$ : additional assumption on the trade-off between return revenue and holding cost) (**: Resource Constrained Shortest

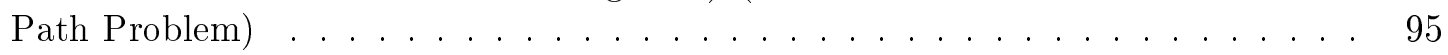

4.1 Illustration of the principle of CRC at a given period $t$ at the 1st and 2 nd decision

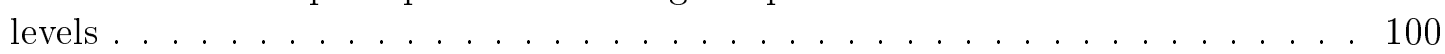

4.2 Possible values of $A_{t}$ and $B_{t}$ in LSP-B-CRC with fixed reserved capacity, a stationary batch size $V$ and $T=7 \ldots \ldots \ldots \ldots \ldots 10 \ldots \ldots \ldots$

4.3 Possible values of $A_{t}$ and $R_{t}$ in LSP-B-CRC with time-dependent reserved capacity being a decision variable, $V=4$ and $T=7 \ldots \ldots \ldots \ldots$. . . . . . . . . . . 103

4.4 Illustration of the principle of DRC at a given period $t$ at the 2nd decision level 104

4.5 Illustration of the principle of TOPC at a given period $t$ at the 2nd decision level 105

4.6 Illustration of the principle of PTDC at a given period $t$ at the 2nd decision level 107

4.7 Illustration of the principle of QFC at a given period $t$ at the 2nd decision level 108

$4.8 \quad$ QFC cost structure at the 2 nd decision level $\ldots \ldots \ldots \ldots \ldots$. . . . . . . . 108 
4.9 Illustration of the principle of $\mathrm{BA}$ at a given period $t$ at the 2nd decision level . . 109

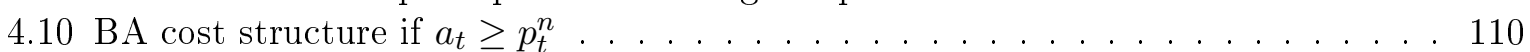

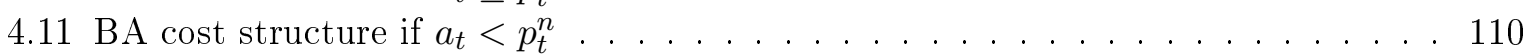

4.12 Cost of batches at a given period $t \ldots \ldots \ldots \ldots \ldots \ldots 11$

4.13 MCC cost structure at the 1st decision level . . . . . . . . . . . . . . . . . . . 112

6.1 Nos contributions pour le contrat buyback . . . . . . . . . . . . . . . . . . 125

$7.1 \quad$ Graph $G^{\prime}$ of Example $3.4 \quad \ldots \ldots \ldots$ 


\section{List of Tables}

2.1 Notations for the general model of the LSP-BR 1 with $w \geq 1 \ldots \ldots \ldots$. . . . . 31

2.2 Costs $\mathscr{L}[t, r]^{u, v}$ in different situations $\ldots \ldots \ldots \ldots \ldots \ldots$. . . . . . . . . 42

2.3 Costs $\mathscr{C}(u, v)$ in different situations $\ldots \ldots \ldots \ldots \ldots \ldots$

2.4 Demand, different costs and revenue data $\ldots \ldots \ldots \ldots \ldots$

$2.5 \quad$ The optimal quantities of $\mathrm{LSP}_{-\mathrm{B}_{F T L} \mathrm{R}_{1} \text { with } w>1} \ldots \ldots \ldots \ldots \ldots$. . . . . . 48

2.6 The optimal quantities of $\mathrm{LSP}_{-\mathrm{B}_{L S, O F B} \mathrm{R}_{1} \text { with } w>1 \ldots \ldots \ldots \ldots} \ldots \ldots \ldots$

$2.7 \quad$ Computational results for DP and MILP of LSP-BR 1 with $w=T(w>1)$. . . . $\quad 59$

2.8 Complexities of the algorithms for different cases studied in Chapter $2 \ldots$. . . . 60

3.1 The optimal quantities of LSP-BD-R ${ }_{1, \rho=1}$ and LSP-BD-R ${ }_{1, \rho=\frac{1}{6}}$ for $V=2 \ldots$. . . 74

3.2 The optimal quantities of LSP-BD-R $R_{1, \rho=1}$ and LSP-BD-R $R_{1, \rho=\frac{1}{9}}$ for $V=4 \ldots$. . . 74

3.3 The optimal quantities of LSP-BD-R $R_{2, \rho=1}$ for $j=1,2,3$ and LSP-BD-R $1, \rho=1: V=479$

3.4 The optimal quantities of LSP-BD-R $\mathrm{R}_{2, \rho=\frac{1}{5}}$ and LSP-BD-R $\mathrm{R}_{2, \rho=\frac{1}{10}}$ for $j=3 . \ldots$. 85

3.5 The optimal quantities of LSP-BD-R $R_{2, \rho=1}$ for $j=3$ and LSP-BD-R $R_{3, \rho=1}, V=4.91$

3.6 Computational results for DP and MILP of the problems with disposal concept . 94

4.1 Notations for the general model of the LSP-B-CRC . . . . . . . . . . . . . . . 99

5.1 Summary table of proposed methods for each problem under buyback contract . 118

6.1 Tableau récapitulatif des méthodes proposées pour chaque problème étudié sous contrat buyback . . . . . . . . . . . . . . . . . 126 


\section{List of abbreviations}

\section{LSP}

LSP-B

$\mathrm{LSP}_{-\mathrm{BR}}$

LSP-BR $_{1}$ with $w \geq 1$

SP-BR 1

LSP-BR $_{1}$ with $w>1$

SP-B ${ }_{O F B} \mathrm{R}_{1}$

SP-B ${ }_{F T L} \mathbf{R}_{1}$

LSP-B ${ }_{O F B} \mathrm{R}_{1}$ with $w>1$

LSP-B ${ }_{F T L} \mathbf{R}_{1}$ with $w>1$

SP-B ${ }_{L S, O F B} \mathrm{R}_{1}$

SP-B ${ }_{L S, F T L} \mathbf{R}_{1}$

LSP-B $_{L S, O F B} \mathrm{R}_{1}$ with $w>1$

LSP-B $_{L S, F T L} \mathrm{R}_{1}$ with $w>1$

LSP-BD-R

LSP-BD-R ${ }_{2}$

LSP-BD-R ${ }_{3}$

LSP-BD-R $_{1, \rho=1}$

LSP-BD-R $_{1, \rho<1}$

LSP-BD-R ${ }_{2, \rho}=1$

LSP-BD-R $2, \rho<1$

LSP-BD-R $_{3, \rho=1}$
Lot Sizing Problem

LSP with batch ordering

LSP-B under the buyback contract with fixed return periods

LSP-BR ${ }_{1}$ with cyclic return periods (at the end of every $w$ periods)

LSP-BR ${ }_{1}$ with returns at the end of each period $(w=1)$

LSP-BR ${ }_{1}$ with returns at the end of every $w$ periods

SP-BR ${ }_{1}$ with only full batch ordering

SP-BR ${ }_{1}$ with full-truck-load cost structure

LSP-BR 1 with $w>1$ and only full batch ordering

LSP-BR 1 with $w>1$ and full-truck-load cost structure

SP-B ${ }_{O F B} \mathrm{R}_{1}$ with lost sales

SP-B ${ }_{F T L} \mathrm{R}_{1}$ with lost sales

LSP-B $\mathrm{B}_{O F B} \mathrm{R}_{1}$ with $w>1$ and lost sales

LSP-B $\mathrm{B}_{F T L} \mathrm{R}_{1}$ with $w>1$ and lost sales

LSP-BR $_{1}$ with disposal

LSP-B with disposal under the buyback contract with a time limit on returns

LSP-B with disposal under the buyback contract with returns only allowed in replenishment periods

LSP-BD-R ${ }_{1}$ with full return

LSP-BD-R $_{1}$ with partial return

LSP-BD-R ${ }_{2}$ with full return

LSP-BD-R $\mathrm{R}_{2}$ with partial return

LSP-BD-R ${ }_{3}$ with full return 
LSP-BD-R $_{3, \rho<1} \quad$ LSP-BD-R R $_{3}$ with partial return

WCSPP Weight Constrained Shortest Path Problem

RCSPP Resource Constrained Shortest Path problem

LSP-B-CRC LSP-B with capacity reservation contract

LSP-B-DRC LSP-B with deductible reservation contract

LSP-B-TOPC LSP-B with take-or-pay contract

LSP-B-PTDC LSP-B with pay-to-delay contract

LSP-B-QFC LSP-B with quantity flexibility contract

LSP-B-BA LSP-B with backup agreement

LSP-B-MCC LSP-B with minimum commitment contract

LSP-B-RSC LSP-B with revenue-sharing contract 


\section{General Introduction}

Since globalization of economies, new technologies and rapid market development lead to increased competition between industries, companies find themselves under pressure to establish strategies for continuously improving their productivity and cost management by responding to ever faster changes in demand. Employing flexible procurement contracts emerges as a recent powerful strategy to attain these goals. Since 1990, there has been a rapid growth in the use of capacity reservation contracts. As a consequence, the supplier allows the buyer, at the contract time, to reserve a certain capacity in advance. At the time of delivery, the buyer decides the number of products to purchase up to the number reserved for an advantageous cost. However, if he requires an amount exceeding the reserved capacity to meet its additional demand, he can get it at a higher cost. Thus, this guarantees flexibility and efficient risk sharing between the supply chain members, in different industries. There are various types of contractual agreements with capacity reservation in the real world: buyback, deductible reservation, take-or-pay, pay-to-delay, quantity flexibility, backup agreement, minimum commitment, and revenue-sharing contracts. In this thesis, we address the capacity reservation contracts, particularly, the buyback contract under several forms and return policies, coupled with batch ordering and inventory management.

The inventory management is a quite old and important area that includes optimal inventory control strategies practiced by several industrial sectors to enhance the product availability and delivery speed, as well as to lead to significant cost reductions, which are crucial elements for the competitiveness. Since decades, the industries have sought to plan either simultaneously or independently the procurement, production, transportation activities over a planning horizon by balancing the costs arising from the storage of products with those of these different activities. According to the US Census Bureau (2013), the inventory value represents huge amounts of money, of about $\$ 1.6$ trillion in the US. Therefore, managing inventories offers an enormous potential to individual companies and to the entire economy. The aim of lot sizing problem is to avoid the excessive inventory build-up in companies and to consist in determining the optimal quantities to order from the supplier (and/or to produce, and/or to transport to the customer) and to store, per product type and per period in order to satisfy the demand with a lowest cost. In our study, we are interested in optimizing the deterministic single-item procurement decision of a retailer over a finite horizon. Traditionally, the ordering policies in inventory management mainly focus on a continuous order size. However, in practical operations, purchased products are delivered in batches, pallets, containers, etc., to the retailer. Another feature of our lot sizing problem is that we consider more general procurement cost patterns: the Full-Truck-Load (FTL) in which the products are replenished in full and fractional batches and the Only-FullBatch (OFB) in which the supplier only delivers in full batches.

In this thesis, there are four chapters. In Chapter 1, we present the literature of procurement contracts and the lot sizing problems with a detailed state of the art by positioning our research within the previous closest studies. In Chapters 2 and 3 , different forms of buyback contract are studied with additional concepts. 
In Chapter 2, we study the lot sizing problem with batch replenishment integrating a periodic buyback contract in which all the unused units are returned to the supplier at the end of every $w$ periods with a unit buyback revenue. The parameter $w$ is fixed in this contract $(w, 2 w, \ldots, N w$ are the cyclic return periods with $N$ being the number of return periods). We consider two return policies: $w=1$ by assuming time-dependent batch sizes, and $w>1$ by assuming a constant batch size over the planning horizon. This problem is investigated with FTL and OFB procurement costs in addition to the classical lot sizing costs. Besides, the concept of lost sales can be allowed in some cases with a cost incurred for each unit of demand not satisfied. The general aim is to determine the procurement, storage and buyback decisions for each extension of this problem. If the lost sales are authorized, we add the lost sales decision. We propose a mixed integer linear program (MILP) for the general case and polynomial time algorithms for the eight extensions considered in this chapter, and we compare the performance of the following resolution methods: MILP and dynamic programming algorithms.

Chapter 3 deals with the procurement planning problem in which the quantity to purchase by a specific form of buyback contract is restricted to a multiple of a constant batch size (OFB). In this chapter, we study three forms of the buyback contract. The first form is the one considered in Chapter 2 but with more general return policies that take into account different periodicity of return periods determined by the two parties $\left(w_{1}, w_{2}, \ldots, w_{N}\right.$ are acyclic return periods with $N$ being the number of returns) and a maximum return percentage fixed by the supplier. The second form only allows returns of units in the first $j$ periods. In the third form, the return periods have to coincide with the ordering periods. For each form, we associate a maximum return percentage $\rho$. For the case $\rho=1$, all the unsold units can be returned. For the case $\rho<1$, only a limited number of unsold units can be returned to the supplier. The values of $w_{i}, \forall i=1, \ldots, N$ (form $\mathrm{n}^{\mathrm{o}} 1$ ), $\rho$ (all the forms) and $j$ (form $\mathrm{n}^{0} 2$ ) are contract parameters. All these lot sizing problems are studied with the disposal concept for two reasons: the first one is that the retailer can have the case $\rho<1$, so he gets rid of the units which cannot be returned, and the second one is that he can have high inventory costs, so he disposes of the units that are not profitable to store and return. Three MILP are proposed for each buyback form. For the three forms with $\rho=1$, and the first one with $\rho<1$, four polynomial time algorithms are developed and are compared with MILP in terms of their execution time. For the second and third forms with $\rho<1$, we can apply the algorithm proposed for the resource constrained shortest path problem with double sided inequality constraints (Beasley and Christofides, 1989).

In Chapter 4, we model the other types of the capacity reservation contract integrated into the lot sizing problem with batch ordering by proposing pseudo-polynomial time algorithms for some problems and leaving the other ones open. We finalize this manuscript with a conclusion and some perspectives in which we give a summary table of all the studied problems under different forms of buyback contract with the hypothesis assumed, complexity results and resolution methods, and, we discuss some possible directions for future research. 


\section{Chapter 1}

\section{Literature review and problem positioning}

The problem studied in this thesis concerns the replenishment planning with batch ordering under a capacity reservation contract in a system with one retailer and one supplier. Indeed, there are several types of capacity reservation contract. We study one of them: the buyback contract, integrated into the single-item lot sizing problem under various hypotheses : lost sales option, possibility of disposal, variable or fixed return periods, etc. Therefore, we can position our research within two existing axes in the literature: supply chain contracts and the lot sizing problem (LSP).

In this chapter, we begin by giving general concepts on different types of procurement contracts, followed by a state of the art of the capacity reservation contract, detailing the buyback contract. Making an exhaustive review of all the research work on the lot sizing problem is far too hard. We give an overview of the LSP to show the huge amount of research work done on the basic lot sizing problem as well as its multiple extensions. After that, we focus on the relevant studies related to the different concepts considered in our problem: LSP with stepwise cost function, LSP with capacity reservation contract, LSP with lost sales and LSP with disposal concept. Finally, we compare our work with the closest studies in the literature to better state our contributions. 


\subsection{Introduction}

A recent trend for many production and distribution systems has been the increase of purchasing volume as a percentage of the company's total sales, which generates a lot of interest in its associated decisions. The purchasing problem is not new. In fact, the National Bureau of Standards in USA recorded the first work addressing this issue dating back to the 1950s before supply chain management became a buzzword and when applications of linear programming were in their beginnings (Stanley et al., 1954). In this context, the buyer-seller relationship receives more importance in order to make products available by following six major purchasing decision processes: make or buy, supplier selection without order quantity allocation, contract negotiation, design collaboration, procurement, and sourcing analysis. In this study, we focus on the procurement process of raw materials and products to be used in a manufacturing environment. Procurement function ensures the purchase of goods, services or works, which are necessary for the activity of the company, from various sources. The role of this function is to optimize the following three components: customer satisfaction (demand, quality, delivery lead time, etc.), the level of inventory and logistics costs. This process requires on the one hand a procurement contract between at least two supply chain members, and on the other hand a replenishment planning model.

We are interested in solving the procurement process at the retailer level considering a special type of capacity reservation contract signed with a supplier to provide quantities in batches. This subject is particularly important to the retail company, as the idea of retail is often associated with the purchase of products which may be transported in containers from suppliers and/or typically arrive at retailing stores in casepacks. The goal of our study is to find optimal planning strategies for this procurement problem under various assumptions by asking various questions. The first one is: What is the type of capacity reservation contract chosen? This question prompted us to give a brief overview of supply chain contracts in Section 1.2, and a detailed literature of the capacity reservation contract and the buyback contract in Section 1.3 and Section 1.4, respectively. The questions related to the procurement planning are: Under which assumptions does the planning problem become harder to solve? If a problem is solved in polynomial time, what is the influence of the different parameters on its complexity? Which methods of resolution can be proposed? What are the most efficient algorithms? These questions allowed us to present in Section 1.5 a general literature review related to the lot sizing problem and in Section 1.6 relevant studies on LSP with different extensions considered in this thesis (piecewise cost function, capacity reservation contract, lost sales and disposal concept). The last question is: What is the difference between our work and the other studies from the literature? This issue incited us to highlight our contributions in Section 1.7.

\subsection{Procurement contracts}

Retailer-supplier collaboration can be defined as a form of cooperation between two non-competing companies. This relationship is nevertheless tense, because each member of the supply chain seeks to optimize its own objective, which can negatively affect the chain and can result in poor performances. However, to obtain a global performance, such a collaboration requires behavioral changes in order to ensure the transparency of relationships, the breakdown of the barriers between company functions, the implementation of methods of management or of control and anticipation of conflicts. The procurement contract plays this role of an anticipatory regulator of the retailer-supplier collaboration in a spirit of sharing risks, resources and gains, and increasing 
the competitiveness of the partners. In this section, we define the procurement contract, present the contracting motivations and finally give a quick overview of the literature on supply chain contracts.

\subsubsection{Definition of procurement contracts}

A contract aims at protecting both contracting parties against any abusive behavior by establishing the obligations of charge and benefit for each one. Thus, a customer engages on demand plans, and cannot derogate from it without paying an additional price to the supplier for each extra unit purchased. Conversely, a supplier may apply rate variations only if these ones are specified in the contract, and agrees on delivery times (Amrani, 2009).

An effective procurement strategy must reduce both the risk of inventory by which we refer to stock shortage or excess, and the risk of price which can be uncertain. The traditional procurement strategy was to use only the long-term contracts which eliminates price risk. In these contracts, the manufacturer and the supplier specify a fixed supply quantity delivered at some time in the future, and, agree on the price. Therefore, this strategy does not present a price risk while it takes a huge risk of inventory because of the impossibility of adjusting the order quantities. Then, the manufacturer takes all the risk. The most recent strategy used in practice to reduce inventory risk is through other types of contracts, for example option contracts or quantity flexibility contracts, which provide the manufacturer with the possibility to adjust the quantities ordered according to the realized demand. Obviously, the unit purchase price paid by the manufacturer in these contracts is typically higher than the price of a long-term contract. The supplier and the manufacturer share the risks.

\subsubsection{Contracting motivations}

There are three large families of reasons that lead partners to engage in a collaborative relationship under contract that we call contracting motivations (Amrani, 2009):

- Uncertainty reduction: The negotiation process initiated during the contractualization aims to conclude an agreement where each of the actors will find a consensual solution to reduce the potential risks: the supplier has a more precise knowledge of the volume of activity associated with his customers and can better adjust its production capacity to exactly their needs; the customer reduces the risk of exposure to an unstable market by negotiating a capacity adaptation with the supplier, and avoids the risk of late delivery.

- Cost control: Often the purchase commitment over a given horizon provides an advantage to both the customer and the supplier. The customer benefits from discounts offered for the purchase of large volumes. He also benefits from the reduction of the renegotiation costs inherent to each punctual purchase and thus ensures a certain stability of purchasing costs over the duration of the contract. The supplier, on his side, has a purchase commitment on a horizon guaranteeing a stable revenue by ensuring the sale of a large volume of products.

- Allocation of responsibility: In a partnership where each party is held to its own obligations, it is important to divide the tasks to be shared and allocate appropriately the responsibility for each party. Each type of responsibility is assigned to the party being the best placed to manage it. The conflicts and litigation situations in the event of non-compliance are thus addressed by pre-determining penalties and financial compensations. 
These motivations shed the light on the success of the partnership relations and justify the supply commitments within a contract. The interview of the head of the Rhodia Group's Purchasing Department, in charge of a portfolio of some thirty suppliers in the field of automation instrumentation and electrical equipment, reveals that the use of procurement procedures under a framework of contract simplifies the workflow. The renegotiations of cost on each order are avoided and this simplification frees up time for buyers to work on specific high value-added actions. On the other hand, considerable economies are realized on the purchase price of grouped volumes. For pressure sensors, for example, a negotiation allows a drop of almost $20 \%$ compared to non-contract prices (Moussay, 2002).

\subsubsection{Research papers on procurement contracts}

The academic literature on supply chain contracts is recent and it can be classified into two categories. The first determines the procurement policies and parameters of a particular contract. The objective of this first category is to optimize the buyer's procurement strategy without analyzing the impact of the decision on the seller. Examples that fall within this class include Brown and Lee (1997), Anupindi and Bassok (1999), Kleinknecht and Akella (2002), and Yazlali and Erhun (2006). The second category focuses on adjustment of the terms of the contract in order to improve the supply chain coordination and the system-wide efficiency. The channel coordination may be achieved by modifying contractually the structure of the relationships between the supply chain members. Unlike the first category, the objective of this second category is to characterize contracts in such a way as to optimize the profit of each party by leading to an optimized supply chain. For instance, see Pasternack (1985), Tsay (1999), and Cachon and Lariviere (2005).

Various forms of contracts between customers and suppliers have been developed to cope with supply chain risks. The best known contracts include long-term contracts (Goldberg and Erickson, 1987), quantity flexibility contracts (Tsay and Lovejoy, 1999), sales rebate contracts (Krishnan et al., 2004), quantity discount contracts (Corbett and Croote, 2000), option contracts (Burnetas and Ritchken, 2002), revenue sharing contracts (Cachon and Lariviere, 2005) and capacity reservation contracts (Serel et al., 2001). For an excellent review on supply chain coordination with contracts see Cachon (2002) and Lariviere (1999) considering several contract types in the supply chain by identifying their benefits and drawbacks, and focusing on single period problems due to their variety and complexity.

\subsection{Capacity reservation contract}

In this thesis, we focus on a capacity reservation contract, between two members of the supply chain, which provides flexibility for a buyer during the replenishment season in order to get additional products from his seller in response to changing market needs. Such contracts are frequently used in toy, apparel, electric power, and high-tech industries (Kleindorfer and $\mathrm{Wu}, 2003)$. These industries are characterized by increased product variety which causes short product lifecycles, forcing companies to shorten lead times in procurement, manufacturing and distribution. Besides, they are confronted to highly unpredictable demands especially in hightech industries such as semiconductors, telecommunications, electronics, and pharmaceuticals. Jin and Wu (2001) reported that the demand volatility can reach $80 \%$ of the average sales during a particular quarter in the case of a telecommunications component manufacturer. Hence, these industries seek to control the risks associated with their characteristics by securing future demands through capacity reservation contracts. For example, Apple's major chip suppliers had reserved a great capacity for the second and third quarters of 2016 for iPhone 7 production 
(Appleinsider, 2017). In the bio-tech sector, many pharmaceutical and biotechnology customers have begun to work with their drug manufacturers to guarantee the needed capacity (Wu et al., 2005). In the following, the capacity reservation contract will be defined by presenting its benefits for the buyer and the supplier. Then, we will provide a considerable literature on this form of supply chain contracting.

\subsubsection{Description and benefits of the capacity reservation contract}

In the general form of the capacity reservation contract, a member X (buyer) of the supply chain first pays a reservation fee to have the right to receive the delivery of the goods from the member $\mathrm{Y}$ (supplier) at a specified date and at a predetermined procurement cost. He receives from the member $\mathrm{Y}$ his ordered quantity, in period $t$, at a price lower than the spot market price, up to a certain capacity. When the ordered quantity exceeds the reserved capacity, the member $\mathrm{X}$ has to replenish the excess amount, but, at a higher cost. Suppose that the fixed cost of the order in period $t$ is $f_{t}$, the capacity is $R_{t}$, the cost to pay for the quantity being less than $R_{t}$ is $a_{t}$, the cost to pay for the quantity being greater than $R_{t}$ is $b_{t}$ and the quantity to order is $x_{t}$. So, to fulfill all the demand of member $\mathrm{X}$, the ordering cost to pay by the member $\mathrm{X}$ is the following one:

$$
\begin{array}{ll}
\mathrm{f}_{t}+a_{t} x_{t} & \text { if } 0<\mathrm{x}_{t} \leq R_{t} \\
\mathrm{f}_{t}+a_{t} R_{t}+b_{t}\left(x_{t}-R_{t}\right) & \text { if } \mathrm{R}_{t} \leq x_{t}
\end{array}
$$

Both parties have benefits. This type of contract provides member X a flexibility to manage uncertain demand and improve capacity planning in the long run, and deliveries without interruption with very advantageous prices for the desired quantity of the reserved capacity (Serel $e t$ al., 2001). In addition, it contributes to mitigate the bullwhip effect of the supply chain (Lee et al., 1997) which can be explained as follows: the final customer places an order, thereafter, the point of sale orders a larger quantity than the final customer demand, to have security stock or to offer discounts on the purchase volume, this creates an increase of quantity to produce at the manufacturer, thus exaggerated fluctuations of orders. Figure 1.1 illustrates this phenomenon in which the orders in the downstream of the supply chain develop to be higher in the upstream of the supply chain. Thus, a small fluctuation of consumer demand can cause greater variations throughout the supply chain.

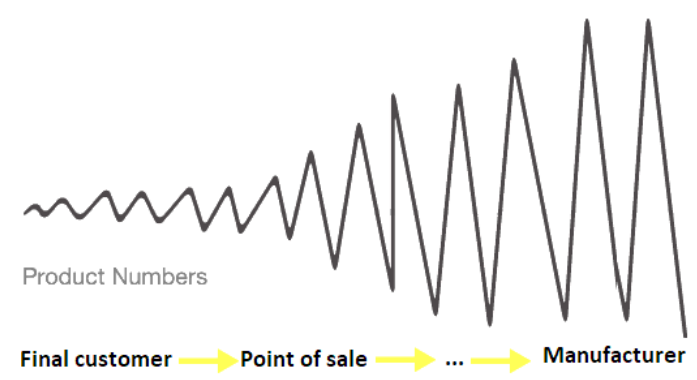

Figure 1.1: Bullwhip effect

For example, the toy maker Mattel reports a $\$ 500$ million sales shortfall in the last weeks of 1998, because it had expected to ship a lot of products to its retailer Toys'R'Us after the 1998 Thanksgiving weekend, but this latter stopped ordering from Mattel because it feared excess inventory based on consumer demands (Kravetz, 1999). The member Y derives benefits from this contractual agreement by establishing a long-term business relationship, reducing transaction costs and offering several profitable prices for the quantities exceeding the capacity. 
According to Park and Kim (2014), the capacity reservation contracts can be classified into two groups: on the one hand, the general contracts which are frequently used in retail and manufacturing industries, and on the other hand, the specialized contracts, which are employed in capital intensive industries. The general contracts include quantity flexibility, backup agreement, buyback, minimum commitment, and revenue-sharing contracts. And the specialized contracts include take-or-pay, pay-to-delay, and deductible reservation contracts. All these contracts will be explained in details in the next chapters.

\subsubsection{Research papers on capacity reservation contracts}

There is a rich body of literature dealing with different procurement problems under capacity reservation contracts. First, there are several research papers which focus on deriving the optimal contract parameters in order to minimize the total supply chain cost. Silver and Jain (1994) and later Jain and Sliver (1995) study the problem in which the buyer pays a capacity reservation cost to the supplier by considering uncertainties in the requirements and supplier capacities. They present conceptual approaches in order to determine the capacities to reserve, period by period, and the periodic replenishment quantities. Costa and Silver (1996) extend these two above mentioned papers to the case where the demand and the supplier capacity are discrete random variables with known probability distributions. In the semiconductor industry, Brown and Lee (1997) study the pay-to-delay capacity reservation (the buyer purchases a minimum quantity at a given price and pays a fee to reserve additional units up to a desired level) with known demand and make the reservation decision for wafer capacity. Kaiser and Tumma (2004) evaluate the take-or-pay contract (the buyer does not pay the reservation fee but he is penalized if he buys a smaller quantity than the reserved capacity) in chemical manufacturing. A synthesis of earlier literature to explain the structure of contracting according to capacity options in capitalintensive industries like chemicals and steel is provided by Kleindorfer and Wu (2003). Wu et al. (2005) claim that with the capacity reservation contracts, the electric power generation company, Gencos, and the distribution company, Discos, will share the risks and also the benefits by appropriately choosing the good contract parameters.

Second, there are various works addressing the design of capacity reservation contracts to achieve channel coordination. Eppen and Iyer (1997) analyze the backup agreements (the buyer commits to purchase a number of units, and the seller agrees to reserve a certain percentage of these units and delivers the remaining) for fashion merchandise between a catalog company (Catco) and manufacturers (Anne Klein, Finity, DKNY, and Liz Claiborne) by proposing a stochastic dynamic programming model. They show that the use of these agreements can lead to profit increase for both parties. Tsay and Lovejoy (1999) model the performance of quantity flexibility contract in supply chains, and show that various contract settings could coordinate the channel when the whole-sale price is adjusted according to flexibility percentages with different profit splits. In the context of high-tech manufacturing, Erkoc and Wu (2005) propose two channel coordination contracts with capacity reservation in a single-period setting: partial payment deduction (a portion of the reservation fee is deductible from the final order payment when the buyer uses the reserved capacity) and reservation with cost sharing (the buyer pays for a part of the capacity ordering cost associated with his reservation and he either receives a refund, or makes additional payment for the capacity utilized according to demand realization). In a similar model setting, Jin and Wu (2007) compare the deductible reservation contract with another capacity reservation contract called take-or-pay to reach channel coordination.

Third, the research on production planning optimization with capacity reservation arised in recent years. Serel et al. (2001) investigate the multi-period sourcing decisions with a stationary 
demand of a company in the presence of a capacity reservation contract that this company signs with its supplier in addition to the spot market alternative. They propose analytic models to study the buyer-supplier relations from the points of view of both parties. Serel (2007) extends the work of Serel et al. (2001) to the case of uncertainties about the quantity of an input item available in the spot market and demand. The author examines the impact of changes in various model parameters on optimal capacity reservation and price decisions made by both buyer and supplier. Inderfurth and Kelle (2013) consider spot price uncertainty without capacity restriction instead of random capacity of spot market at a given price considered by Serel (2007). They develop a stochastic dynamic programming approach for analyzing and solving the structure of the optimal combined purchasing policy. Park and Kim (2014) propose a linear programming model for a capacity reservation contract with multi-period and single/multi-supplier settings. The authors suggest a rolling-horizon implementation strategy in order to handle the real life contracts. These papers mainly assume a linear reserved capacity cost. Very limited literature focuses on the production planning problem with nonlinear capacity costs; we cite for example the work of Huang et al. (2018). In these research papers which address the multi-period production planning problem, the authors characterize the optimal production strategies for a given capacity reservation level, and then, determine the buyer's optimal reserved capacity. In this work, we study a lot-sizing problem with a capacity reservation contract. After the explanation of the lot-sizing problem in Section 1.5, we will give the related references explored in the literature.

\subsection{Buyback contract}

This work is concerned with a special type of capacity reservation contract: buyback contract in which the buyer has the possibility to return a certain percentage of the unused items to the supplier for a revenue that is less than or equal to the procurement cost. In such a contract, the buyer procures $Q$ units for a fee $p$, and receives a revenue, $p^{b}$, for each unit not utilized. This is analogous to a capacity reservation contract with a reservation and procurement of $Q$ units at a cost $p-p^{b}$, and a procurement of $Q-N$ units of excess quantity for a fee, $p^{b}$ ( $N$ is the unused capacity). In the following section, we will present some applications of the buyback contracts and relevant research from the literature.

\subsubsection{Buyback contract applications}

The buyback contract arises in many industrial settings: any product with a limited life time due to physical decay (dairy products, baked goods, pharmaceuticals, cosmetics), products with a risk of obsolescence (fashion apparel, computer hardware and software, greeting cards, magazines, newspapers), and products with high carrying costs or products with rapidly saturated demands (books and recorded music). The form of buyback contract varies in and across industries (Pasternack, 1985; Hou et al., 2010). The following are some real life examples of buyback contracts, found in the literature:

- IBM implements the buyback contract to help distributors better manage their personal computer inventories (Shi and Xiao, 2008).

- McKesson, a major distributor of health and beauty products, offers retailers a return program trading off more generous return policies at higher purchase prices (Padmanabhan and Png, 1995). 
- Wahmpreneur Books which sells books to retailers and wholesalers which may return them for full credit within a 30 day timeline (Bose and Anand, 2007).

- The booksellers return between 30 and $35 \%$ of new hardcover books to the publisher (Cachon and Terwiesch, 2006). This form of buyback contract comes with partial return.

- In book distribution, Prentice Hall gives retailers a refund that can be applied to subsequent purchases (Padmanabhan and Png, 1997).

- Kids Wholesale Wearhouse sells children's clothes to retail outlets and allows for returns with a refund which is worth $25 \%$ of restocking fee (Bose and Anand, 2007). This form presents a partial refund.

- Procter \& Gamble pays back its retailers the unsold inventory value of the product "Bainde-soleil" at the end of summer (Padmanabhan and Png, 1997).

- Big State, an independent distributor of recorded music, allows retailers to return up to $15 \%$ of the quantity purchased within 40 days following the purchase month, thereafter, no returns are accepted (Padmanabhan and Png, 1995).

\subsubsection{Description of the buyback contract}

A buyback contract involves three parameters $\left(p, p^{b}, \rho\right)$, with $p$ being the procurement cost per unit, $p^{b}$ being a pay back revenue $\left(0<p^{b} \leq p\right)$, and $\rho$ being the maximum return percentage $(0<\rho \leq 1)$. Under such a mechanism, the supplier sells $x$ units to a buyer at a cost $p$ per unit and allows the customer to return a maximum of $\rho x$ at the end of the selling season with a revenue $p^{b}$ per unit. The manufacturer encourages retailers to order more to satisfy uncertain demand. If $p^{b}=p$, the contract is said to be full refund, otherwise it is called partial refund. If $\rho=1$, the retailer can return all the unsold units so the contract is said to be full return. Otherwise $(\rho<1)$, the retailer can only return a limited number of unsold units to the supplier, so the contract is said to be partial return.

\subsubsection{Literature review on the buyback contract}

In the literature, the buyback contract is mostly used in the context of single period planning. According to Hou et al. (2010), there are three factors that lead to use this type of contract: properties of the product (perishability, single or multi-item patterns, risk attitude of the supply chain members, etc.), demand pattern (uncertainty, price dependence, etc.), and supply chain structure (single or multi-level, type of channel, etc.).

The representative articles of the first factor are numerous. Pasternack (1985) is the seminal work regarding buyback contracts for a coordination perspective between a manufacturer and retailers. The author considers a setting in which a manufacturer produces a perishable commodity in a single period. He shows that the supply chain can be coordinated either by partial returns with full buyback revenue or by full returns with partial buyback revenue. For multi-item buyback policy, we find the work of Brown et al. (2008) in which the distributor can return any combination of the products up to a certain proportion of the total procurement of all products. The authors analyze the distributor's optimal profit, and compare it to the case of a single-item returns policy. Choi et al. (2008) address the issues of channel coordination and risk aversion of single-supplier and single-retailer. The authors formulate a mean-variance model for a supply chain with a single product in a single time period, and they show that a buyback contract can 
help channel coordination and control risk. Xiong et al. (2011) study a composite contract based on risk aversion for a two-stage supply chain by combining two contracts: a buyback contract and a quantity flexibility contract. They show that this contract has advantages over supply chain coordination, profit allocation, and risk allocation. See also Luo et al. (2018) who address the operational decisions and coordination of the supply chain in the presence of risk aversion.

The problem under returns policy with demand uncertainty has been investigated by Marvel and Peck (1995) who affirm that the manufacturer's decision to accept returns depends on the nature of the demand uncertainty. Emmons and Gilbert (1998) show that the supplier can improve channel profits if demand variability follows a uniform distribution. Hou et al. (2010) study a buyback contract between a buyer and a backup supplier under supply disruption. They investigate the differences of the contract parameters under backup supply uncertainty and demand uncertainty. Yao et al. (2005) study the impact of the factor of demand information sharing on a return policy and the profits of both parties under different scenarios, and analyze the effects of key parameters on their profits. The price-sensitive demand factor in a return policy contract is analyzed by Yao et al. (2008) in a single-period supply chain. The authors present an analytical model to identify the contract properties. A group of scholars have also examined return policies under this factor (Marvel and Peck, 1995; Emmons and Gilbert, 1998; Lee, 2001; Arcelus et al., 2008).

The third category of the buyback literature focuses on the supply chain structure. Song et al. (2008) analyze a buyback contract, in a two-echelon Stackelberg framework, between a manufacturer and a price setting newsvendor retailer. Cachon and Lariviere (2005) study the supply chain coordination with revenue-sharing contracts by comparing them to the buyback contract. Bose and Anand (2007) study properties of return policies in the framework of a single period stochastic model either with an exogenously fixed wholesale price or unilaterally declared price by one dominant party. Ding and Chen (2008) investigate the coordination of a three level supply chain with flexible return policies selling short life cycle products in a single period model. Yao et al. (2005) examine the design of the returns in a manufacture-retail supply chain consisting of a mix of a traditional retail channel and a direct channel.

In a large number of papers (above papers; Krishnan et al., 2004; Wang and Zipkin, 2009; $\mathrm{Wu}, 2013$; etc), the buyback contract has been studied for its design and implementation in different supply chain configurations. To the best of our knowledge, there has been no research that studies a production planning problem under a buyback contract. In this work, we will integrate the buyback contract with different return policies between a retailer and a supplier in a multi-period LSP.

\subsection{Overview of lot sizing problems}

The lot sizing problem (LSP) is encountered in procurement, production, and transportation planning. Its purpose is to determine the quantities to produce (to order or to transport) for each item over a given finite horizon in order to satisfy the demand at a lowest cost. Apart from a fixed setup cost (or fixed ordering cost), there are also unit production (replenishment) and unit storage costs incurred. The amounts of product are called "lot" and calculated to regroup the demands of different periods in order to be produced (procured or transported), in advance, if this reduces the total production costs, especially the fixed setup cost. However, early production generates inventories and, if inventory costs are high, it may be more interesting to allocate production over several periods which induces more setups but less storage. The LSP is therefore often equivalent to finding the best compromise between the setup costs and inventory 
costs.

One of the ancestors of the LSP models is the economic order quantity (EOQ) model proposed by Harris in 1913. Its objective is to minimize the total replenishment and inventory carrying costs for an infinite planning horizon. The model considers a single item, deterministic static demand, continuous time, and uncapacitated case. Unlike this continuous problem, Manne (1958) and Wagner and Whitin (1958) are the first works on the discrete LSP which assumes that the planning horizon is finite and divided into discrete periods for which the demand is given and may be dynamic. Following these first studies, the number of LSP papers published in the academic literature grew very strongly under different extensions.

In this section, we give, first, the basic model of the lot sizing problem along with its classical formulation as a mixed integer linear program (MILP). Second, we present the characteristics of the lot sizing problem such as: stochastic demand, bounded inventories, production capacity, lost sales, batch production, etc. Third, we identify review papers, and finally, we cite the used solution methods.

\subsubsection{Modeling the basic problem}

The problem we present is the single item, single level uncapacitated lot sizing problem, known as Wagner and Whitin (WW) problem which aims to determine the production planning over a horizon that is discretized into $T$ periods. The goal is to decide how much to produce in each period $t$ and how many units to store from period $t$ to period $t+1$ in order to satisfy the known demand $d_{t}$ by minimizing the total production and holding costs. The production cost is composed of two terms: a fixed setup cost $f_{t}$ independent of the quantity produced, and a unit cost $p_{t}$ incurred for each produced unit. The inventory holding costs are classically modeled by unit costs $h_{t}$ for each unit stored at the end of each period $t$. All these costs can be timedependent, stationary, or can have arbitrary patterns under various assumptions. The demand for each period must be entirely satisfied without backlogging through the production or the stocks. The decision variables are: $x_{t}$, the quantity to produce in period $t, y_{t}=1$ if a setup occurs in period $t\left(x_{t}>0\right)$ and zero otherwise, and $s_{t}$, the inventory level at the end of period $t$ $(t=1, \ldots, T)$. This problem can be used to plan the procurement or transportation activity.

The WW problem can be formulated as follow:

$$
\begin{aligned}
& \min \sum_{t=1}^{T}\left(f_{t} y_{t}+p_{t} x_{t}+h_{t} s_{t}\right) \\
& \text { subject to } x_{t}+s_{t-1}=d_{t}+s_{t} \quad \forall t=1, \ldots, T \\
& x_{t} \leq\left(\sum_{j=t}^{T} d_{j}\right) y_{t} \quad \forall t=1, \ldots, T \\
& x_{t}, s_{t} \in \mathbb{R}_{+}, y_{t} \in\{0,1\} \quad \forall t=1, \ldots, T
\end{aligned}
$$

The objective function (1.1) is to minimize the total production and inventory costs over the horizon of $T$ periods. Constraints (1.2) represent the flow conservation, that is, the entering stock of the period $t\left(s_{t-1}\right)$ added to the amount produced in $t\left(x_{t}\right)$ are used to satisfy the demand $\left(d_{t}\right)$, and what remains is kept in stock at the end of the same period $\left(s_{t}\right)$. Constraints (1.3) indicate a relation between the production quantity and the setup variable in which a production at period $t$ incurs a setup cost. Constraints (1.4) define the feasibility domain of the continuous variables $x_{t}$ and $s_{t}$ and the binary variables $y_{t}$.

This formulation is called aggregate because the production is only defined by a period of production, in contrast with disaggregated formulations such as facility location-based formulation in which the production is defined by a period of production and a period of consumption.

The well known ZIO (Zero-Inventory Ordering) policy is developed by Wagner and Whitin (1958) for non-speculative cost structure $\left(p_{t}+h_{t} \geq p_{t+1}\right.$ for any period $t \in\{1, \ldots, T-1\}$, 
which means that producing one unit in $t$ and storing it till the following period costs more than producing it in $t+1$ ), and based on the principle that it is not optimal to produce during a period $t$ if the stock at the end of period $t-1$ is non-zero $\left(s_{t-1} x_{t}=0, \forall t=1, \ldots, T\right)$. This property allows to solve the WW problem by a dynamic programming algorithm in $O\left(T^{2}\right)$ time for linear variable ordering and holding costs. This algorithm has been improved by Wagelmans et al.(1992), Federgruen and Tzur (1991), and Aggarwal and Park (1993), independently to an $O(T \log (T))$ time algorithm in the general case and to an $O(T)$ time algorithm under nonspeculative costs. In these models, several criteria are not inserted, which means that these solutions are not, in many cases, feasible at the operational level. To model several real-life situations, different extensions of the WW problem have been studied in the literature.

\subsubsection{Characteristics of the lot sizing problem}

Various extensions of the basic LSP have been developed over the years according to different parameters. We present the most used characteristics in the literature, by relying on the classifications of LSP proposed by Haase (1994) and Brahimi (2004).

\section{Planning horizon}

This attribute represents the time interval for planning the production. It may be either infinite or finite. It considers changes in model parameters over time: an infinite horizon is usually accompanied by stationary parameters (e.g. Papachristos and Ganas, 1998). However, a finite horizon is coupled by dynamic parameters. In our models, almost all the parameters are assumed to be dynamic which means that they vary over time.

\section{Information degree}

This attribute describes whether uncertainty is considered or not in the model, then we have: the deterministic case (all parameters are known in advance) vs. the stochastic case (some or all parameters are not exactly known and are based on some probabilities). See Tarim and Kingsman (2004) and Guan et al. (2006) for more details on stochastic problems. In our models, we assume that all the parameters are deterministic.

\section{Time scale}

The time scale is another important characteristic that affects the lot sizing decisions. It can be either discrete or continuous. For the discrete-type system, the LSP falls into the categories of either small time bucket (Drexl and Kimms, 1997) or big time bucket problems (Van Hoesel et al., 1994). The small time bucket problems are characterized by the fact that the time period is so short and that only one item can be produced in each time period (hour, day). While, for the big time bucket problems, the time period is long enough, so that multiple items can be produced in the same time period. The continuous time models are addressed by Hanssmann (1962), Lopez and Kingsmans (1991), and, Yao and Elmaghraby (2001).

\section{Number of items}

There are two types of LSP in terms of number of items: single item or multi-item LSP. For example, van Hoesel and Wagelmans (2001) develop theoretical results for fully polynomial approximation schemes for single item capacitated LSP, and Hindi et al. (2001) propose Lagrangian 
relaxation with subgradient optimization combined with a smoothing heuristic and local search in order to solve multi-item capacitated LSP with setup times.

\section{Number of levels}

The LSP may be single level or multi-level. In the single level LSP (Stadtler, 2000), the final product is directly processed from raw materials by a single operation (e.g. forging or casting). The demand of this product is named independent demand. In multi-level systems (Lee et al., 2003; Zhang et al., 2012), there is a parent-component relationship defined in the bill of material of products. Raw materials go through several operations to become final products. The demand at one level is known as dependent demand. The complexity of multi-level LSP is higher than the one of single level LSP.

\section{Relevant costs}

In addition to the unit production cost, there are other costs to take into account in the LSP model. First, there is the startup cost which is incurred when the producer switches from one item to another and prepares the resource for the new one. They differ from the setup cost which is generated whenever the resource is enabled for production. Wolsey (1989) and van Hoesel et al. (1994) study the uncapacitated single item LSP with startup costs by proposing different classes of valid inequalities. Second, there are costs of reservation which are incurred in the case where the resource is kept in a given state even if it remains vacant during several periods. Toy and Berk (2006) develop an $O\left(T^{3}\right)$ algorithm for the capacitated single item LSP in a warm/cold process in which the process can be kept warm from period $t$ to period $t+1$ by paying a reservation cost. Third, there are the inventory holding costs which correspond to the costs of blocked capital, risk of loss or obsolescence, taxes, insurance and warehouse maintenance operations. Fourth, there are the backorder (stockout) costs. The backlogging aims to satisfy the demand, but later than the initially required period. This extension was first studied by Zangwill (1969) to solve the uncapacitated single item LSP by proposing an $O\left(T^{2}\right)$ algorithm reduced to $O(T \log T)$ algorithm. Fifth, there are lost sales costs, which will be detailed in the next section since some of the LSP models that we address in the present work are based on this concept. Sixth, there are costs related to capacity: costs of regular capacity (regular hours) or costs of additional capacity (additional hours, subcontracting). See Özdamar and Bozyel (2000) for LSP overtime decisions. There are also transportation costs related to batches, trucks, etc., which are stepwise. For more details on these costs, refer to Section 1.6. Into lot sizing problems, we can integrate many other costs to model many other extensions.

\section{Resource constraints and their nature}

In the literature there are many papers on LSP with an infinite amount of available resources (manpower, machines, inventory, lot sizes, budget, etc.) to simplify its resolution, while this assumption is not realistic in many practical situations. Hence, the extension to the resource constraint case needs to be integrated into LSP in which the resource can be single or multiple, and constant or variable. The resource constraints can be: production capacity constraint (Van Hoesel and Wagelmans, 1996), bounded storage constraint (Akbalik et al., 2015), perishable inventory constraint (Hsu, 2000), minimum order quantities constraint (Okhrin and Richter, 2011), and, constant batch sizes constraint (Akbalik and Pochet, 2009). The complete and/or incomplete batch ordering assumptions are the resource constraints of this study. These extensions will be discussed in Section 1.6. 


\section{Service Policy}

In order to avoid problems affecting the quality of customer service, a safety stock can be used as protection against uncertainties of demand, lead time, and procurement. Subcontracting (buying some goods from outside), backlogging (satisfy late demands in relation to delivery time), time windows, and lost sales are possible solutions added to the LSP in the event of out of stock.

\section{Time consuming activities}

These include for instance, the setup time, processing time (zero, constant, variable), lead time, and transportation time.

\section{Objectives}

The goal of the LSP is either to minimize the total cost, or to maximize the service level, or to smooth the production load, or to maximize the profit.

The literature contains a plethora of classification of the LSP extensions proposed by several review papers cited below.

\subsubsection{Surveys of lot sizing problems}

Reviewing the literature of the LSP has become increasingly popular over the last 15 years. Readers can refer to Glock et al. (2014) who show the increase in the number of review papers published per year between 1974 and 2012. The work of Glock et al. (2014) is a tertiary study which identifies and analyzes literature reviews of LSP till 2012. The authors categorize the reviews of works on LSP along two aspects: aspect of technical structures and content-related aspect (see Table B1 in Glock et al., 2014). In relation to technical structure, we find stationary models vs. dynamic models, and deterministic models vs. stochastic models. For the contentrelated aspect, there are classical models whose objective is to find the optimal order, production and shipment quantities and extended models which consider other types of decisions, in addition to the ones of LSP (such as scheduling, worker learning, perishable inventory, etc.).

Since different cases we study in this manuscript are dynamic deterministic single level LSP, we cite some reviews addressing this field. Karimi et al. (2003) consider single and multiple item and production capacity constraint, and study all the solution approaches. Brahimi et al. (2006) consider the single item, uncapacitated version without and with extensions (backlogging, perishable inventory, remanufacturing, time windows, etc.) and the capacitated version, present their different mathematical programming formulations and review the solution techniques for the capacitated version. Jans and Degraeve (2008) focus on the modeling of various LSP industrial extensions organized around two aspects: operational aspects (setups, production, inventory, and demand) and, tactical and strategic aspects (for example, integrated production-distribution planning, supplier selection, etc.).

Although the literature is abundant in reviews on LSP, more surveys are continuously appearing in the literature, to bring more updates in this field and to address new extensions. Pahl and Voß (2014) consider deterioration and lifetime constraints in production and supply chain planning models. Biel and Glock (2016) discuss the articles studying the energy-efficient LSP in order to respect the new environmental standards and energy consumption. Copil et al. (2017) present the literature on dynamic simultaneous lot sizing and scheduling problems. Brahimi et al. (2017) focus on publications of single item dynamic LSP with all the possible extensions from 2004 to 2016 by surveying their complexity and solution procedures. 


\subsubsection{Resolution methods}

Based on the literature, there are two types of the LSP solution approaches for the deterministic case. On the one hand, we find exact methods which aim at determining the optimal solution of the problem. On the other hand, there are heuristic or approximation methods which aim at obtaining the best possible solution, without guarantee of optimality.

The exact methods include dynamic programming, valid inequalities and Branch and Cut approaches, Branch and Bound algorithms, and strong formulations (formulation without inventory variables, facility location-based formulation, shortest path formulation). For the uncapacitated single item LSP with demand time windows and backlogging, Hwang (2007) proposes an $O\left(T^{3}\right)$ dynamic programming algorithm by considering a non-speculative cost structure. The effectiveness of this method is based on the optimality principle of Bellman (1957): any optimal policy is composed of optimal sub-policies. Leung et al. (1989) use Branch and Cut algorithm in which they study the polyhedral structure of the single item and constant capacity LSP by developing several valid inequalities. They obtain a good description of the convex hull of feasible solutions to this problem. Chung et al. (1994) propose an exact algorithm that combines dynamic programming with Branch and Bound to solve the capacitated single item LSP with linear production and holding costs and non-speculative costs. This combined method consists in determining an optimal solution through a tree of enumeration by progressive separation and evaluation. Fatemi Ghomi and Hashemin (2002) consider the single item resource constrained production problem with constant setup cost and develop an analytical method based on reformulating it as a shortest path problem. Under certain assumptions, their algorithm presents optimal solutions. Wagelmans et al. (1992) use the facility location-based formulation for the WW problem to derive their dynamic programming algorithm whose idea is to identify the optimal non-production periods. It is noted that dynamic programming is the most used method to solve the LSP.

For more complex lot sizing problems (NP-hard problems) in which extensions like multiitem, multi-level, production capacity, etc., are included, heuristic methods have been developed either because researchers did not find exact methods, or to obtain a reduced calculation effort than that of the exact approaches. These approaches can be classified into Period-by-period heuristics, improvement heuristics, meta-heuristics (tabu search, simulated annealing, genetic algorithms), mathematical programming-based heuristics and Lagrangian heuristics.

\subsection{Relevant references related to our study}

In our study, we focus on the single item LSP under a capacity reservation contract with batch ordering by proposing exact methods such as dynamic programming. We consider a piecewise cost structure for the batch cost. We decide to integrate the buyback contract into an LSP. In some problems, we add the possibility of lost sales where demand is not satisfied and in others, the option of disposals of unsold and unreturned units. In the following, we give the important articles being the closest to our topic along four axes, which position this work within the LSP literature. The first is constituted by the studies on the LSP with a stepwise cost function. The second axis consists of the LSP with capacity reservation contract. The third examines the works on LSP with lost sales. Finally, we present the references dealing with the LSP with disposals. 


\subsubsection{Lot sizing problem with stepwise cost function}

A stepwise or piecewise concave function is composed of a sequence of concave functions in the $m$ subintervals $\left[V^{0}, V^{1}\right],\left[V^{1}, V^{2}\right], \ldots,\left[V^{m-1}, V^{m}\right]$ with $V^{j}$ for $j=0, \ldots, m$ being the price breakpoints of the global function and $V^{0}<V^{1}<\cdots<V^{m}$. It can be continuous or discontinuous. The piecewise procurement (or production or transportation) cost functions can be used to represent quantity discounts, capacity acquisition, subcontracting, overloading, minimum quantity requirements and capacities, batch ordering/production/delivery as well as a combination of these latters (Koca et al., 2014). We cite some examples of stepwise costs considered in the literature (see Figure 1.2).

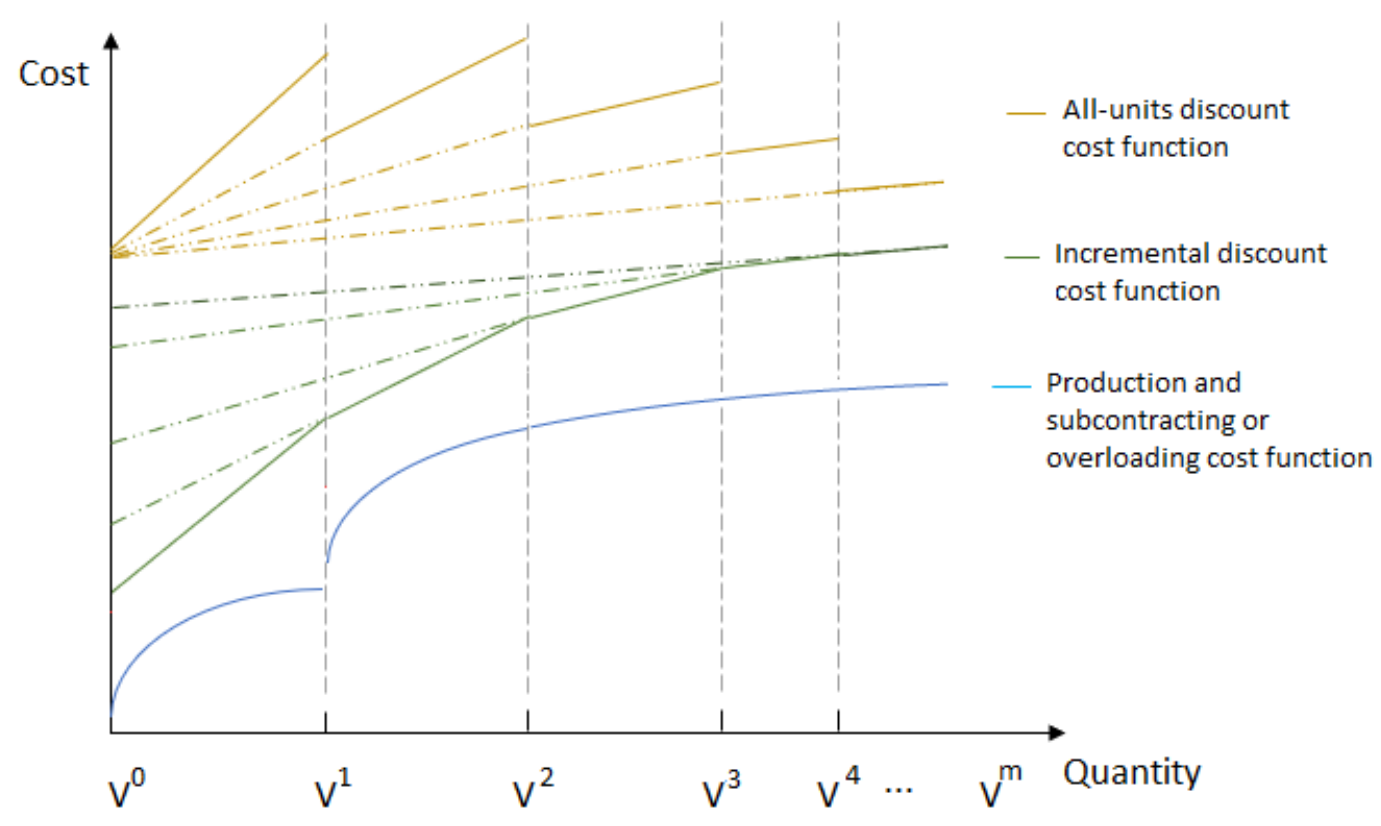

Figure 1.2: Some examples of stepwise concave functions

In the LSP with quantity discounts, we find two popular types of piecewise cost functions used to present discount pricing schemes. The first one is the all-units discount cost function (see Figure 1.2, brown curve) under which different discount rates are applied to all units of different purchased quantities. Li et al. (2012) study the uncapacitated single item LSP with all-units discount and resales over a planning horizon of $T$ periods under the assumption that the breakpoints of the cost function are stationary. The authors show that this problem, with the number of discount price breakpoints being arbitrary, is NP-hard. For the case where the number of breakpoints, $m$, is fixed, they develop a polynomial algorithm with an $O\left(T^{m+3}\right)$ running time. The second type is the incremental discount cost function (see Figure 1.2 green curve) under which different discount rates are applied to incremental ranges of the ordered quantity. Archetti et al. (2014) consider the single item LSP with incremental discount (an increasing piecewise linear function with no flat sections) without capacity constraints. The authors propose an $O\left(T^{2}\right)$ time algorithm knowing that the number of breakpoints is bounded.

In the LSP with subcontracting, the overall production \& subcontracting cost (presented in Figure 1.2 blue curve) follows a discontinuous piecewise concave function composed of two pieces: the first piece of the function corresponds to regular production with unitary low cost and a capacity $V^{1}$, and the second piece corresponds to subcontracting with unitary high cost 
and without capacity. Atamturk and Hochbaum (2001) study the LSP with subcontracting and capacity acquisition in order to satisfy deterministic non-stationary demand over a finite horizon knowing that the inventory holding cost is a linear function. The authors suppose that the capacity is stationary whether being deterministic or being a decision variable. The capacity cost only depends on the capacity. For the case with concave production and subcontracting costs, they develop $O\left(T^{5}\right)$ and $O\left(T^{8}\right)$ time algorithms for the case with known capacity and for the case with the capacity being a decision variable with a concave capacity cost, respectively.

In the LSP with batch ordering (or production or delivery), the products must be ordered in batches (pallets, containers, vehicle or trucks). Then, in addition to fixed and variable procurement costs, there is a piecewise replenishment cost related to batches. This cost has the Truck-Load (TL) cost structure (see Figure 1.3. Figure 1.4 and Figure 1.5 under which a Less-Truck-Load (LTL) rate (positive slope) is paid until the buyer pays for the cost of a FullTruck-Load (FTL) of $V$ units (flat section), which is less than the cost of delivering $V$ units at an LTL rate (dotted positive slope). Once the first truck is full, the LTL cost is paid until the second truck is full, and so on.

The generalized TL cost function in which the LTL cost follows a non-linear concave function is shown in Figure 1.3. It is also called Lippman piecewise cost function being discontinuous. Indeed, Lippman (1969) is the first researcher to consider a single item uncapacitated LSP with batch ordering without setup cost nor backlogging where the transportation, procurement and holding costs depend on time and the batch size is constant. The author proposes an $O\left(T^{5}\right)$ time algorithm.

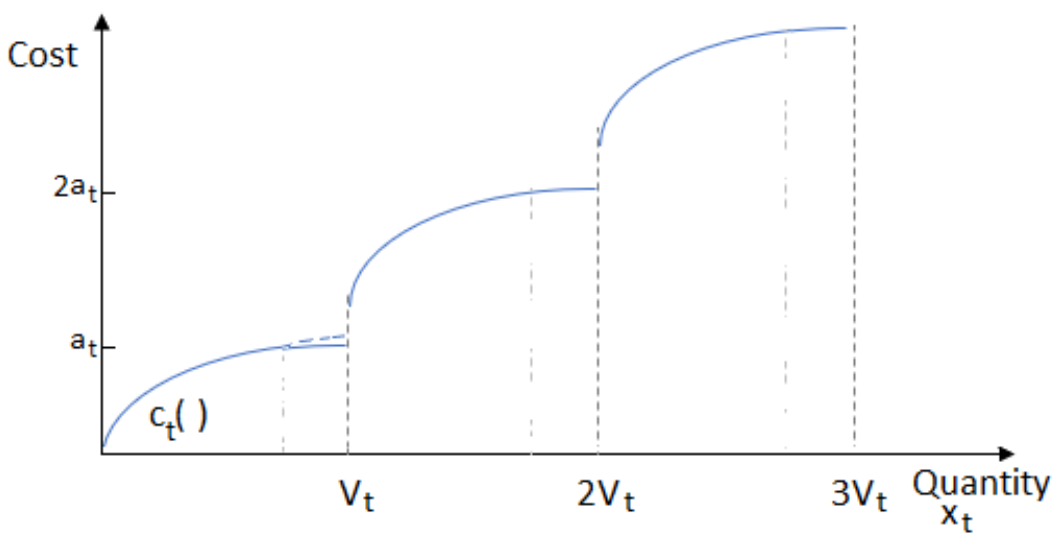

Figure 1.3: Generalized TL cost function

Let $\alpha$ be a real number, so $\lceil\alpha\rceil$ denotes the smallest integer that is greater than or equal to $\alpha$ and $\lfloor\alpha\rfloor$ indicate the largest integer that is smaller than or equal to $\alpha$.

Under this structure, products must be ordered at period $t$ in batches of $V_{t}$ units and the buyer pays the LTL cost $c_{t}$ for the units being in an incomplete batch and the FTL cost $a_{t}$ for a full batch. The overall procurement cost $q_{t}\left(x_{t}\right)$ for a positive amount $x_{t}$ of products ordered in period $t$ which is composed of a fixed setup cost $f_{t}$, a purchase cost $p_{t}$, an LTL cost $c_{t}$, and an FTL cost $a_{t}$, is given as follows:

$$
q_{t}\left(x_{t}\right)=f_{t}+p_{t}\left(x_{t}\right)+a_{t}\left\lfloor\frac{x_{t}}{V_{t}}\right\rfloor+\min \left\{c_{t}\left(x_{t}-\left\lfloor\frac{x_{t}}{V_{t}}\right\rfloor V_{t}\right), a_{t}\right\}, q(0)=0
$$

In the literature, there are two special cases of the generalized TL cost structure according 
to the nature of LTL and FTL costs. The first one is the linear TL cost function in which the LTL cost follows a non-decreasing linear function as shown in Figure 1.4. Li et al. (2004) address two uncapacitated LSP with batch ordering, setup cost, time varying cost parameters and backlogging. In the first problem, the authors assume that the procurement quantities are limited to multiples of a constant batch size and they develop an algorithm in $\mathrm{O}\left(T^{2}\right)$ time improved to $O(T \log (T))$ time through the use of Monge matrices. In the second problem which is more general than the first one, they include the transportation costs which have the linear TL cost structure which means that the replenishment quantities may not be a multiple of the constant batch size. An $\mathrm{O}\left(T^{3} \log (T)\right)$ time algorithm is developed to solve this problem. Besides, they extend these two problems in order to take into account non-decreasing concave holding, backordering, and LTL freight cost functions (generalized TL cost function) and they prove that the first two proposed algorithms can also be applied to solve these extended problems. Hence, the authors improve the Lippman's algorithm.

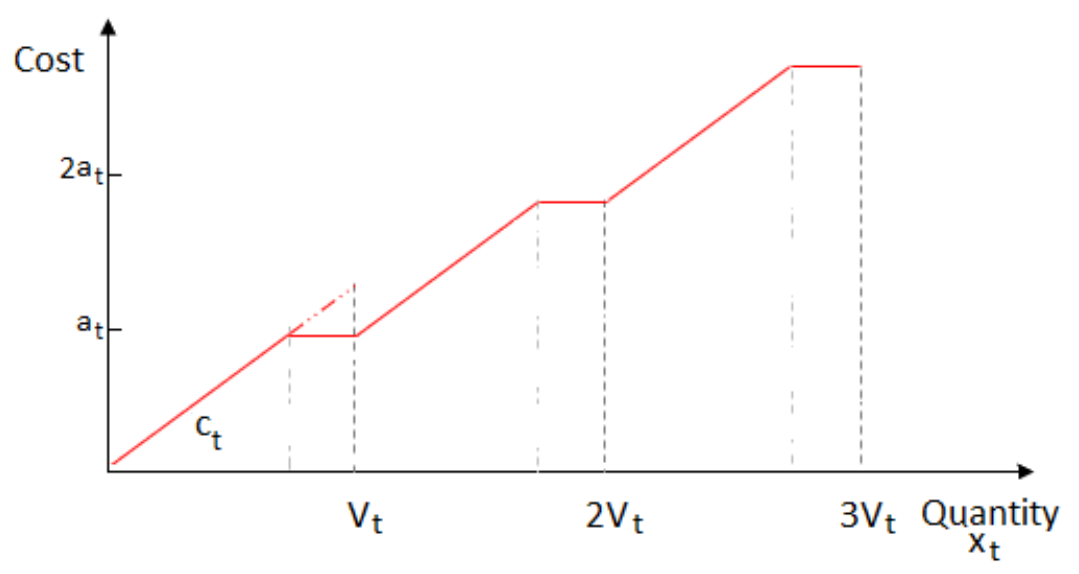

Figure 1.4: Linear TL cost function

The second one is the TL cost structure with infinite LTL cost (Figure 1.5), called FTL cost structure which is considered in this study. Under the FTL cost structure, the buyer pays a fixed cost per batch replenished (or produced or transported) even if the batch is not complete. The total procurement cost for a positive quantity $x_{t}$ is equal to:

$$
q_{t}\left(x_{t}\right)=f_{t}+p_{t}\left(x_{t}\right)+a_{t}\left\lceil\frac{x_{t}}{V_{t}}\right\rceil, q(0)=0
$$

Figure 1.5 illustrates the stepwise cost structure for null unit cost and Figure 1.6 shows the total procurement cost function considered in this study.

In order to mention the works on LSP with FTL cost structure, we need to use the notation $\left(f_{t}, p_{t}, h_{t}, a_{t}, V_{t}\right)$ which designates the assumptions adopted in this problem knowing that $f_{t}$ : setup cost in period $t, p_{t}$ : unit procurement cost in period $t, h_{t}$ : unit holding cost in period $t$, $a_{t}$ : fixed cost per batch replenished in period $t$ and $V_{t}$ : batch size in period $t$. If a parameter $\beta$ along these is null, then we assign to it the value '-', if it is assumed constant, then we write it without subscript $t, \beta$, and if it is time-dependent, we keep the period $t$ in its notation and in this case, if it is allowed to be linear, we write $\beta_{t}$ and if it is concave, then we write $\beta_{t}()$. For example, the notation $\left(-, p_{t}(), h_{t}, a_{t}, V\right)$ corresponds to an LSP without setup cost, with concave unit procurement cost, linear holding cost, linear fixed cost per batch and stationary batch size. 


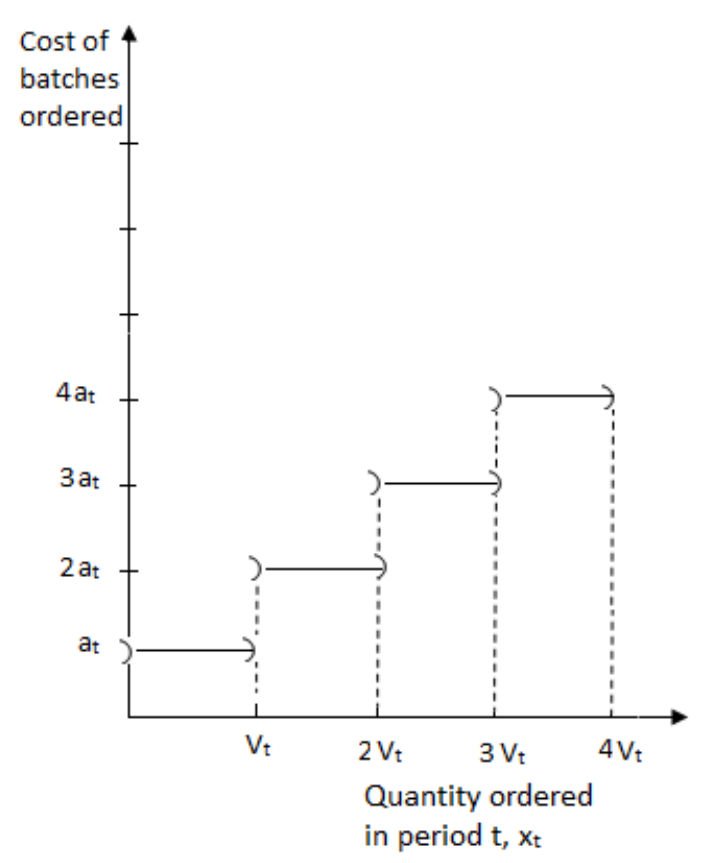

Figure 1.5: FTL cost function

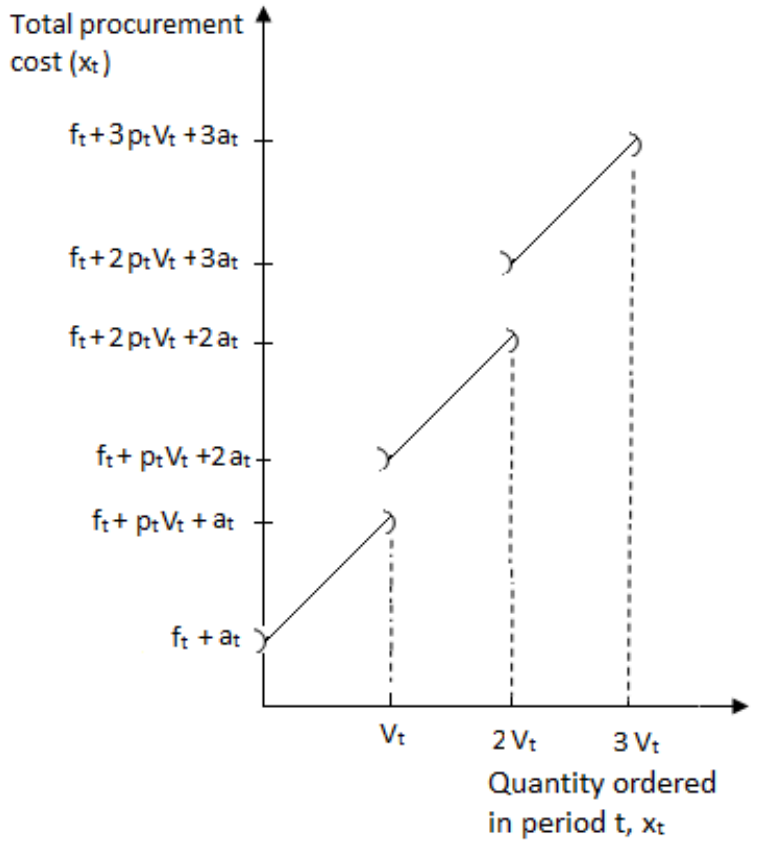

Figure 1.6: Total procurement cost function

The $\mathrm{O}\left(T^{5}\right)$ time algorithm developed by Lippman (1969) can solve the LSP with FTL cost structure in which the author assumes $\left(-, p_{t}, h_{t}, a_{t}, V\right)$. After that, Lee (1989) proposes an $O\left(T^{4}\right)$ time algorithm for the case $(f, p, h, a, V)$. Pochet and Wolsey (1993) study the LSP of Lippman with FTL cost structure by improving the algorithm complexity to $\mathrm{O}\left(T^{2} \min (T, V)\right)$ time. For more general assumptions $\left(f_{t}, p_{t}, h_{t}(), a_{t}, V\right)$, the $\mathrm{O}\left(T^{3} \log (T)\right)$ time algorithm proposed by Li et al. (2004) can be used to solve the LSP with FTL cost structure being also a special case of the linear TL cost function, knowing that the authors add concave backlogging costs. Hwang (2010) provides for the problem with $\left(f_{t}, p_{t}(), h_{t}(), a_{t}, V\right)$ an $\mathrm{O}\left(T^{5}\right)$ time algorithm and with backlogging an $\mathrm{O}\left(T^{6}\right)$ time algorithm. Hwang and Kang (2016) develop a two phase algorithm running in $\mathrm{O}\left(T^{2} \log (T)\right)$ time for the case $\left(f_{t}, p_{t}, h_{t}, a_{t}, V\right)$ with no-speculative-motives in production and backlogging. Akbalik and Rapine (2018) study the LSP with multi-mode replenishment (multiple suppliers) and a stationary batch size. The same fixed cost is paid for whatever the number of units replenished in a batch. The authors prove that the general problem is NP-hard and they propose an $O\left(T^{4}\right)$ time algorithm for the case $\left(f_{t}, p_{t}(), h_{t}, a_{t}, V\right)$ with a single mode.

Notice that all of those studies assume a stationary batch size. Akbalik and Rapine (2013) address the uncapacitated LSP with time-dependent batch sizes $V_{t}$. The authors study the complexity of this problem according to the behavior in time of the cost parameters (setup cost, unit procurement cost, unit holding cost, and fixed cost per batch). Therefore, they prove that the LSP with FTL cost structure for the case of time-varying batch sizes is NP-hard if one of the cost parameters is time-dependent. Under the assumption $\left(f,-,-, a, V_{t}\right)$, they show that the problem is polynomially solvable in time $O\left(T^{3}\right)$.

Researchers have extended the LSP with FTL cost structure in various settings by making some simplifications to propose polynomial time algorithms. Anily and Tzur (2006) consider the problem of shipping multi-items by identical capacitated vehicles $\left(V_{t}=V\right)$ with a stationary setup cost per vehicle dispatched. The unit shipping and inventory holding costs are itemdependent but they are constant over time. The authors develop two algorithms for solving 
the problem optimally, based on a dynamic programming approach and an exponential search method. Jaruphongsa et al. (2007) consider a two echelon dynamic LSP with two outbound delivery modes where the first mode has a fixed setup cost structure while the second has an FTL cost structure. The aim of this problem is to minimize the system-wide cost of replenishment, inventory holding, and transportation with alternative shipment modes between the upper (warehouse) and lower (distribution center) echelon. All the cost parameters are stationary except the unit holding cost at the warehouse and at the distribution center. The authors provide an $O\left(T^{5}\right)$ time algorithm based on a dynamic programming approach. Van Vyve $(2007)$ addresses the capacitated LSP with batch delivery and develops an $O\left(T^{3}\right)$ time algorithm for the case $\left(-, p_{t}, h_{t}, a_{t}, V\right)$ with non-speculative costs, time-dependent production capacity and allowing backlog. Without backlogging, the algorithm time complexity reduces to $\mathrm{O}\left(T^{2} \log (T)\right)$. Akbalik and Rapine (2012) study a similar problem, but with a non null setup cost, namely $\left(f_{t}, p_{t}, h_{t}, a_{t}, V\right)$, and with stationary production capacity and without backlogging. The authors propose two polynomial time algorithms: an $O\left(T^{4}\right)$ time algorithm for the case with production capacity being a multiple of the batch size and an $O\left(T^{6}\right)$ time algorithm for the case of an arbitrary capacity. Akbalik and Penz (2011) study the single item capacitated LSP with time window deliveries, two echelon and FTL cost of transportation. The authors show that this problem is NP-hard even by assuming the same time window value for all time periods. Ou and Feng (2019) consider an LSP with backlogging and capacity adjustment. The authors assume that, in each period, there are $m$ different production capacity levels available to be acquired. Each production capacity level is assumed to be a multiple of a base capacity unit $V$ (FTL cost structure with $m$ breakpoints, where $m$ represents the maximum number of batches to replenish). In this problem, the production costs are dependent on the capacity acquired in each period, and there is a capacity adjustment cost if the capacity acquired for period $t$ differs from the one of period $t-1$. They develop an $O\left(m^{4} T^{4}\right)$ time algorithm for the general case $\left(f_{t}, p_{t}(), h_{t}(), a_{t}, V\right)$. In addition to the extensions of production capacity, time window, etc., there are works which address the problem with FTL cost structure, where the buyer signs a capacity reservation contract with the supplier that allows him to reserve a certain capacity in advance and to buy it for an advantageous price.

\subsubsection{Lot sizing problem with capacity reservation contract}

Consider a lot sizing problem where the retailer places orders to satisfy a deterministic demand over a planning horizon of $T$ periods following a capacity reservation contract. In the general setting of this contract, the retailer reserves a capacity $R_{t}$ in each period $t$ from the supplier with a reservation fee $r_{t}, t=1, \ldots, T$. In return, the supplier offers the retailer in period $t$ quantities up to the reserved capacity $R_{t}$ at a unit replenishment cost $a_{t} . x_{1, t}$ is the number of regular units ordered in period $t$ being less than $R_{t}$. In the same period $t$, if the retailer desires to procure a quantity which exceeds the reservation capacity $R_{t}$, then he gets the excess quantity $x_{2, t}$ at a unit price $b_{t}$ from the same supplier. The cost $b_{t}$ can also be considered as a price of the spot market. It is assumed to be higher than the reservation fee $r_{t}$ plus the regular procurement cost $a_{t}$, then we have $r_{t}+a_{t}<b_{t}$. If $r_{t}+a_{t} \geq b_{t}$, then the reserved capacity $R_{t}$ is zero and the procurement of period $t$ is insured with the cost $b_{t}$. This situation does not coincide with the practical applications of capacity reservation contracts.

For any positive amount ordered $x_{1, t}+x_{2, t}$, a setup cost $f_{t}$ is paid. There is an inventory holding $\operatorname{cost} h_{t}$ incurred for each unit of the inventory level $s_{t}$ at the end of time period $t$. Hence, the demand in period $t, d_{t}$, can be satisfied from procurement with $a_{t}$ cost, with/without procurement with $b_{t}$ cost in that period and/or from inventory. The LSP with capacity reservation contract 
(LSP-CRC) can be either a tactical problem or an operational problem. In the tactical model, the reserved capacity is a decision variable (general model), while in the operational model, it is given. We present the general formulation of LSP-CRC whose objective is to minimize the capacity reservation, purchasing and inventory costs by finding in each period $t$ the optimal values of $R_{t}, x_{1, t}, x_{2, t}, y_{t}$ and $s_{t}$.

$$
\begin{aligned}
& \min \sum_{t=1}^{T}\left(r_{t} R_{t}+f_{t} y_{t}+a_{t} x_{1, t}+b_{t} x_{2, t}+h_{t} s_{t}\right) \\
& \text { subject to } x_{1, t}+x_{2, t}+s_{t-1}=d_{t}+s_{t} \quad \forall t=1, \ldots, T \\
& x_{1, t} \leq R_{t} \\
& x_{1, t}+x_{2, t} \leq\left(\sum_{j=t}^{T} d_{j}\right) y_{t} \\
& \forall t=1, \ldots, T \\
& \forall t=1, \ldots, T \\
& x_{1, t}, x_{2, t}, s_{t} \in \mathbb{R}_{+}, R_{t} \in \mathbb{N}, y_{t} \in\{0,1\} \quad \forall t=1, \ldots, T
\end{aligned}
$$

Constraints (1.5) represent the balance of inventory. Constraints (1.6) ensure that the total quantity replenished at a cost $a_{t}$ is limited by the reserved capacity. Constraints (1.7) force the variables $y_{t}$ to take the value of 1 if the retailer procures a positive quantity from the supplier. This capacity reservation model can also be adapted to the production planning model with capacity acquisition and subcontracting or overtime options. The cost $r_{t}$ is equivalent to the capacity acquisition cost. The cost $a_{t}$ corresponds to the regular production cost. The extra cost $b_{t}$ is incurred due to an overtime production or a quantity outsourced without any fixed cost. Therefore, the capacity reservation contract procurement cost structure corresponds to an overall production \& subcontracting linear cost structure (see Figure 1.7 in which $a_{t}<b_{t}$ because $r_{t}+a_{t}<b_{t}$ ), which means that it is a piecewise function. Then, the LSP-CRC belongs to the lot sizing problems with piecewise production cost functions.

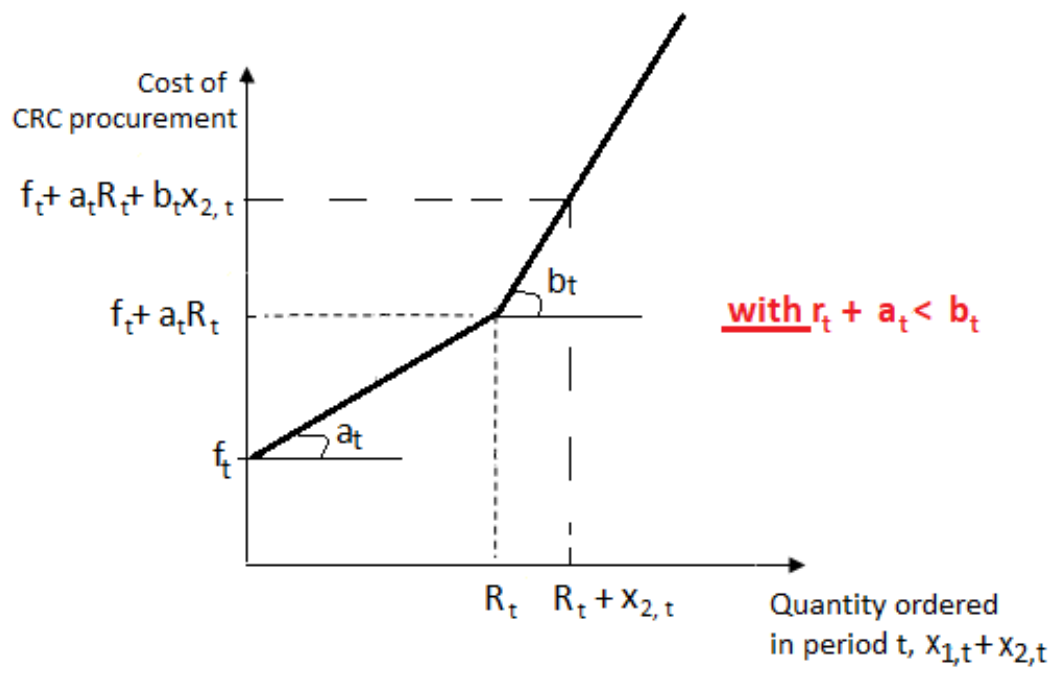

Figure 1.7: Capacity reservation cost structure

Atamturk and Hochbaum (2001) consider a single item LSP to study the stationary capacity expansion, uncapacitated subcontracting, production and holding inventory decisions for a finitehorizon demand satisfaction problem. The production capacity $R_{t}=R$ needs to be determined in some problems and it is fixed (given) in others. The authors assume different cost structures for $a_{t}$ and $b_{t}$ (non-speculative linear, non-speculative fixed-charge, and concave cost structures) and without any relationship between them. The capacity cost is stationary, $r_{t}=r$, but it 
can follow a concave function $(r(R))$ which depends on $R$. For non-speculative production and subcontracting costs, they propose an $O\left(T^{2}\right)$ time algorithm for the operational LSP-CRC $(R$ is given, $f_{t}$ is positive, production $a_{t}$ and subcontracting $b_{t}$ costs are linear) and an $O\left(T^{5}\right)$ time algorithm for the tactical LSP-CRC ( $R$ is a decision variable, $f_{t}$ is positive, capacity, production and subcontracting costs are linear).

In the previous formulation of LSP-CRC with given reserved capacities, we consider the case where, in each period $t$, the unit cost of extra procurement $b_{t}$ is relatively higher than the one of the regular procurement $a_{t}$. Thus, in this contract, extra procurement happens only if the regular procurement is already at full reserved capacity.

Lee and $\mathrm{Li}$ (2013) consider the single-item dynamic LSP-CRC where the reserved capacity $R_{t}$ is a given parameter for all the periods $t$ from 1 till $T$, the reservation cost is stationary $\left(r_{t}=r\right)$, and with $a_{t}<b_{t}, \forall t \in\{1, \ldots, T\}$. The authors affirm that the LSP-CRC is a more general problem than the classical capacitated LSP (CLSP) which is obtained by setting, in LSP-CRC, the extra costs $b_{t}=+\infty, \forall t \in\{1, \ldots, T\}$ and by ensuring that $\sum_{j=1}^{T} d_{j} \leq \sum_{j=1}^{T} R_{j}$ to have a feasible solution.

In a CLSP, the quantity to be produced (procured, distributed or sold) of single or multiple items at each period of the horizon is limited by a certain capacity. It seems that the first work on these problems started with the article of Manne (1958) which studies the production scheduling problem faced by a machine shop required to produce many different items. The papers of Florian et al. (1980), and Bitran and Yanasse (1982) prove that the complexity of the single item CLSP depends primarily on the structure of setup, inventory and production costs and capacities. These problems are generally NP-hard, thus many heuristics and relaxations are designed in the literature. However, some cases of the CLSP can be solved in polynomial time such as Chung and Lin (1988) developing an $\mathrm{O}\left(T^{2}\right)$ time algorithm for the CLSP with nonincreasing $f_{t}$ and $a_{t}$ costs, general $h_{t}$ costs and non-decreasing capacities $R_{t}$, over the horizon. Besides, van Hoesel and Wagelmans (1996) propose an $\mathrm{O}\left(T^{3}\right)$ time algorithm, for the case where $\operatorname{costs}\left(f_{t}, h_{t}, a_{t}\right)$ are arbitrary and the production capacity is stationary $(R)$. Literature reviews of CLSP can be found in Karimi et al. (2003) and Buschkühl et al. (2010) for multiple items and in Brahimi et al. (2006) for the single item case.

Despite the NP-hardness of the LSP-CRC, Lee and Li (2013) focus on the two previous models which are known to have polynomial time algorithms in the classic CLSP, by adding the spot price $b_{t}$ for all periods of the horizon. They propose $\mathrm{O}\left(T^{3}\right)$ and $\mathrm{O}\left(T^{4}\right)$ time algorithms for the LSP-CRC with Chung and Lin (1988)'s assumptions and non-increasing $b_{t}$ costs and for the LSP-CRC with Van Hoesel and Wagelmans (1996)'s assumptions and general $b_{t}$ costs, respectively.

Zhang (2015) studies the same LSP-CRC under a constant reservation capacity $R$ considered in Lee and $\mathrm{Li}(2013)$ but with positive fixed extra procurement (or outsourcing) costs $f_{t}^{\prime}$, for all periods $t=1, \ldots, T$. The author proposes an $O\left(T^{5}\right)$ time dynamic programming algorithm. The same problem under non-speculative costs is solved in Atamturk and Hochbaum (2001) by an $O\left(T^{3}\right)$ time algorithm but no relationship between unit regular and extra procurement costs is assumed. Phouratsamay (2017) addresses the two-level LSP-CRC with inventory bounds in a supply chain composed of one supplier and one retailer. The author proposes a complexity analysis and dynamic programming algorithms for different assumptions considered.

All the above studies assume unitary costs in the capacity reservation contract instead of fixed costs per batch (FTL cost structure). In the LSP with batch production under capacity reservation contract, denoted by LSP-B-CRC, the retailer reserves, in each period $t, R_{t}$ batches from the supplier with a cost of $r_{t}$ per batch. In each period $t$, the number of regular batches ordered, denoted by $A_{t}$, at a cost of $a_{t}$ per batch is less than the reservation capacity $R_{t}$. 
The number of extra batches ordered, denoted by $B_{t}$, is at a cost of $b_{t}$ for each excess batch replenished. The total number of batches replenished contains $x_{t}$ units, knowing that the batch size is $V_{t}$. For any positive quantity ordered, $x_{t}>0$, the retailer has to pay a setup cost $f_{t}$ in addition to a unit replenishment cost $p_{t}$ for each unit ordered. In period $t$, the overall ordering cost $q_{t}\left(x_{t}\right)$ (see Figure 1.8) for $x_{t}$ units ordered in $A_{t}+B_{t}$ batches with a reservation of $R_{t}$ batches is presented as follows:

$$
q_{t}\left(x_{t}\right)=f_{t}+p_{t} x_{t}+a_{t} A_{t}+b_{t} B_{t}, \text { with } x_{t} \leq V_{t}\left(A_{t}+B_{t}\right) \text { and } A_{t} \leq R_{t}, q(0)=0
$$

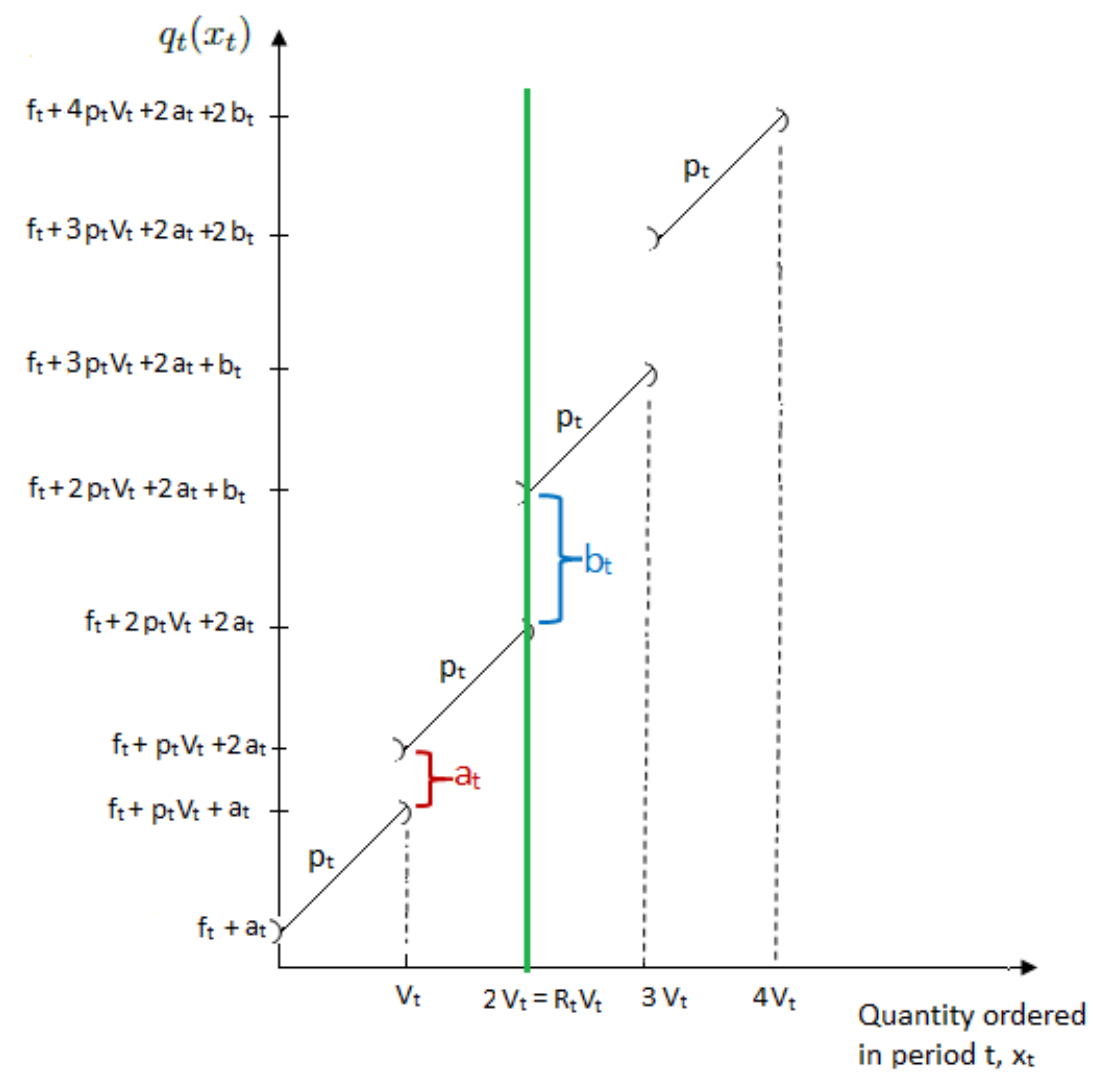

Figure 1.8: Capacity reservation contract cost function

The different formulations of the LSP-B-CRC are detailed in the last chapter. A few papers study this problem.

Van Norden and van de Velde (2005) are the pioneer who investigate the LSP-B-CRC. They study the multi-item transportation problem with dynamic setup costs $f_{i, t}$ which are itemdependent, dynamic holding costs $h_{t}$, null unit procurement costs $p_{t}=0, \forall t=1, \ldots, T$, stationary transportation costs per batch composed of the low freight cost $a$ and the high shipping cost $b$, a constant batch size $V$ and a defined reservation capacity $R$. A batch can contain different items. They propose a Lagrangian heuristic to solve this strongly NP-hard problem.

Molina et al. (2009) develop a method consisting of a Lagrangian/surrogate heuristic to solve the problem presented in van Norden and van de Velde (2005) with backlogging and production time capacity constraints. Refer to Molina et al. (2016) for extensions of the model originally proposed in van Norden and van de Velde (2005). 
Akbalik et al. (2017) study different classes of LSP-B-CRC by generalizing the total procurement cost in which the authors do not assume a relationship between the exceeding batch costs and the regular batch costs. According to the assumptions adopted on the given parameters $f_{t}, p_{t}, h_{t}, a_{t}, b_{t}, R_{t}$ and $V_{t}$, they identify NP-hard cases and propose a pseudo-polynomial time dynamic programming algorithm and several polynomial time algorithms for other cases.

\subsubsection{Lot sizing problem with lost sales}

Lost sales mean unsatisfied demands where the retailer has to decide whether or not to satisfy the entire demand or only a portion within a period. It is possible to serve only one part of the demand, if this is more beneficial. Companies having low demand for certain products with low profit in a certain period can find it more profitable to lose this demand. Besides, in many practical situations, firms allow the backlogging of demand (postponed demand) for a given number of periods, but if the delivery date is too late, the order can be canceled. The lost sales incur a direct cost to the firm, as well as an indirect cost. The direct cost corresponds to the lost revenue of the canceled demand. The indirect cost is a penalty related to the loss of customer goodwill at a later time, which may be explained by a decline in future demand. It represents the unknown impact of poor service. Hence, the cost of lost sales is greater than the selling price.

In lot sizing, the classical decision of how much and when to order is extended to the decision of when and which fraction of demand to lose over the horizon. To obtain the formulation of LSP with lost sales (LSP-LS), we have to add in the basic model of WW, a non-negative decision variable of the unmet demand at the end of each period $t, L_{t}$, and a unitary lost sales cost $l_{t}$ at period $t$, knowing that we have $l_{t} \geq p_{t}, \forall t=1, \ldots, T$. The uncapacitated LSP-LS problem can be formulated as follow:

$$
\begin{aligned}
& \min \sum_{t=1}^{T}\left(f_{t} y_{t}+p_{t} x_{t}+l_{t} L_{t}+h_{t} s_{t}\right) \\
& \text { subject to } x_{t}+L_{t}+s_{t-1}=d_{t}+s_{t} \quad \forall t=1, \ldots, T \\
& L_{t} \leq d_{t} \quad \forall t=1, \ldots, T \\
& x_{t} \leq \sum_{j=t}^{T} d_{j} y_{t} \quad \forall t=1, \ldots, T \\
& x_{t}, L_{t}, s_{t} \in \mathbb{R}_{+}, y_{t} \in\{0,1\} \quad \forall t=1, \ldots, T
\end{aligned}
$$

In the objective function (1.9), we have to minimize the total lost sales costs over the $T$ periods in addition to the costs depicted in the objective function (1.1). Constraints (1.10) take into account the lost sales quantity in the inventory balance. Constraints (1.11) ensure that any canceled demand $L_{t}$ in period $t$ cannot exceed the demand $d_{t}$ of that period.

The model of LSP-LS is equivalent to the one of production LSP with bounded outsourcing. In each period, the level of outsourcing is limited by the demand. In these models, the overall in-house production \& outsourcing cost is not piecewise. Its cost function is neither concave nor convex. The retailer can outsource with the $\operatorname{cost} l_{t}$ in such a period without producing with the regular procurement costs, $f_{t}$ and $p_{t}$ (see periods 1 and 3 in Figure 1.9). If regular production and holding inventory for a number of time periods are cheaper than outsourcing, then it is reasonable to produce (see period 2 in Figure 1.9). In period 4, the demand is satisfied by the inventory of the previous period 3. Thus, this problem differs from the operational LSP-CRC detailed in Subsection 1.6.2.

A few papers on lot sizing problems with lost sales or with outsourcing modeled as lost sales are published in the literature. Sandbothe and Thompson (1990) study a problem that is related to the case of lost demand in LSP: a capacitated LSP-LS without limited lost sales quantity. All cost parameters and production capacity are assumed to be constant. They propose an $\mathrm{O}\left(T^{3}\right)$ 


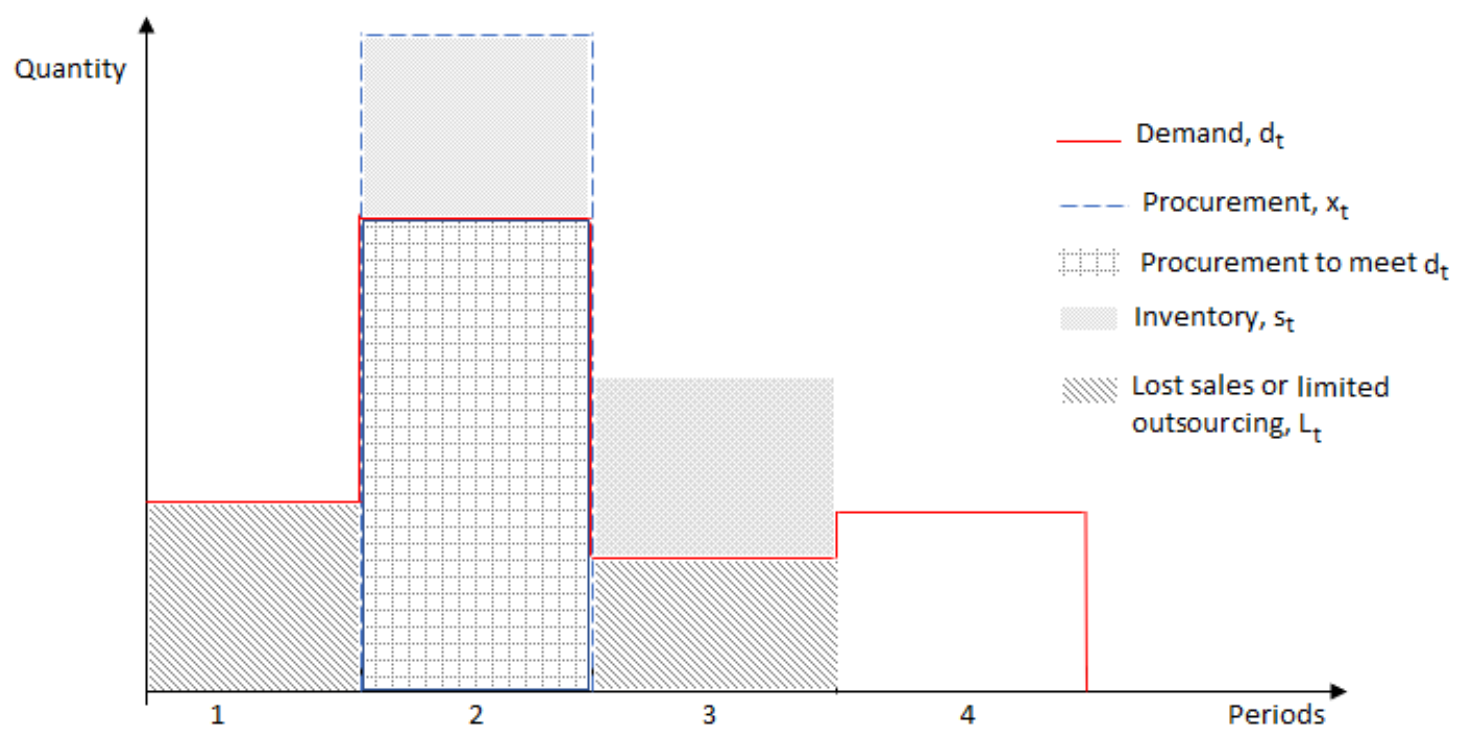

Figure 1.9: Optimal solution of an example of LSP-LS

time forward dynamic programming algorithm to solve it optimally. Aksen et al. (2003) consider an uncapacitated single item LSP-LS and propose an $\mathrm{O}\left(T^{2}\right)$ time dynamic programming algorithm for the time-varying costs. Aksen (2007) considers an LSP-LS in which the unsatisfied demand in a given period causes the demand in the next period to shrink due to the loss of customer goodwill. The author develops a neighborhood search and restoration heuristic. Chu and Chu (2007) develop an $\mathrm{O}\left(T^{2}\right)$ algorithm to solve the LSP with entirely or partially outsourcing of the demand with linear holding and outsourcing cost functions, and linear production cost functions with fixed charges.

In Absi et al. (2011), an uncapacitated LSP-LS with production time windows and early production is studied. The early production means that the demand is satisfied from a production that is processed before the release period of the demand. They propose an algorithm running in $\mathrm{O}\left(T^{2}\right)$ time. The LSP-LS has also been studied by Absi et al. (2013) for the multi-item capacitated case with setup times and lost sales. The authors propose a Lagrangian heuristic based on a probing strategy and a refining procedure to find feasible solutions. And they also develop a metaheuristic based on the adaptive large neighborhood search to improve solutions. Hwang et al. (2013) provide an $O\left(T^{4}\right)$ dynamic programming algorithm for the uncapacitated LSP-LS with upper bounds on stocks for time-varying storage capacities, and production, holding and lost sales costs. Chu et al. (2013) consider the single item uncapacitated LSP with bounded outsourcing, backlogging and limited inventory capacity. The backlogging level at each period cannot exceed a certain upper limit. The authors propose an $O\left(T^{4}\right)$ for the case with linear production cost functions, a fixed cost, and linear holding, backlogging and outsourcing cost functions.

\subsubsection{Lot sizing problem with disposals}

In several application areas, the retailer can dispose of the unsold quantity in a variety of ways, such as discounts, outlet stores, donation to charities, throw in the trash, returning to the supplier through a buyback contract, etc. In this case, the disposal represents a profit for a retailer. The case with non-negative disposal revenue per unit is considered as the resale in the literature. 
However, in some situations, disposal can represent a cost rather than a revenue because it may incur costs associated with transportation, disassembly and separation of hazardous materials, etc.

In the literature, there are two major categories of lot sizing problems into which the disposal option is integrated. The first category includes the LSP with manufacturing and remanufacturing planning. Since 1990, there is a growing body of literature addressing this issue. Remanufacturing can be defined as the recovery of returned items (a given parameter) from the customer to the retailer. These products can be reused, either directly or after this reverse logistics operation. The main difference between this problem and the classical LSP is that the demand of each period at the retailer can be satisfied either by newly produced items and/or by used items being remanufactured. The disposal concept considered in these problems concerns the excess inventory of returned products.

To the best of our knowledge, Richter and Weber (2001) were the first to extend the WagnerWhitin problem to consider the remanufacturing with disposal option of some of the returned products. The same problem with less restrictive assumptions was addressed by Golany et al. (2001). The authors prove that the general problem with concave cost functions is NP-hard and they also provide an exact algorithm of $O\left(T^{3}\right)$ time complexity for the case of linear costs. For more details on the LSP with remanufacturing and disposal, refer to Piñeyro and Viera (2009, 2010).

The second category consists in LSP with quantity discount in which the retailer may face a situation where it is economical to purchase a large quantity in excess of the demand and then dispose of any leftover inventory at a salvage revenue or even at a cost. The discounted unit costs of the product (slopes in subintervals $\left[V^{j-1}, V^{j}\right], j=1, \ldots, m$ with $m$ being the number of price breakpoints, as shown on Figure 1.2 brown and green curves) decrease as a function of the price breakpoints $V^{j}$. In this model, the retailer has to make a relationship between the procurement cost and the non-negative disposal revenue because he can purchase an unlimited quantity in some period $t$, carry it, and resell it after this period to obtain an unlimited profit, and the problem has an unbounded optimum.

The LSP with quantity discount and disposal is obtained from the WW problem in which the non-negative quantity $x_{t}$ is purchased in period $t$ with a product cost that has an all-units discount or incremental discount structure $p_{t}\left(x_{t}\right)$, in addition to the fixed ordering cost $f_{t}$. The retailer may choose to dispose of part of the inventory, denoted by $e_{t}$ and receive a revenue of $p_{t}^{e}$ per unit which can be positive, zero, or negative, $\forall t=1, \ldots, T$. The uncapacitated LSP with quantity discount and disposal can be formulated as the following mathematical program, in which we add the component of disposal revenues over $T$ periods and introduce the amount of disposed products $e_{t}$ in the constraints of inventory balance:

$$
\begin{aligned}
& \min \sum_{t=1}^{T}\left(f_{t} y_{t}+p_{t}\left(x_{t}\right)+h_{t} s_{t}-p_{t}^{e} e_{t}\right) \\
& \text { subject to } x_{t}+s_{t-1}=d_{t}+s_{t}+e_{t} \quad \forall t=1, \ldots, T \\
& x_{t} \leq \sum_{j=t}^{T} d_{j} y_{t} \quad \forall t=1, \ldots, T \\
& x_{t}, s_{t}, e_{t} \in \mathbb{R}_{+}, y_{t} \in\{0,1\} \quad \forall t=1, \ldots, T
\end{aligned}
$$

This problem has received very little attention in the academic literature. Sethi (1984) considers the LSP with all-units discounts with a single price breakpoint $\left(V^{0}=0\right.$ and $\left.V^{1}>0\right)$ and allows the possibility of disposal at a finite unit cost with a constant demand rate. The case with negative disposal cost (resale revenue) per unit is modeled by Sohn and Hwang (1987). These authors study a similar problem with time-varying setup, regular unit cost (first slope), holding costs, resale revenues and stationary discount rate (second slope). They develop a 
pseudo-polynomial algorithm and show that their algorithm can be generalized to solve the multiple breakpoint problem. Mirmohammadi and Eshghi (2012) reduce the time complexity of the algorithm proposed by Sohn and Hwang (1987) by developing an algorithm with an $O\left(T^{4}\right)$ running time. Li et al. (2012) study the LSP with all-units discount and resales with a fairly general setting. The authors assume dynamic unit discount cost rates with stationary price breakpoints. The disposal revenue can be positive, zero, or negative. They propose an $O\left(T^{2}\right)$ algorithm for the case of a single price breakpoint and an $O\left(T^{m+3}\right)$ algorithm for the case of $m$ fixed price breakpoints.

\subsection{Our contributions}

The contributions of this thesis to the literature are shown in Figure 1.10 . Our first contribution is to solve a deterministic LSP under a buyback contract, which is, to the best of our knowledge, not yet explored in the literature. However, there are a few papers on the LSP under the capacity reservation contract $(\mathrm{CRC})$, which consists in a general form of the buyback contract (Atamturk and Hochbaum, 2001; van Norden and van de Velde, 2005; Lee and Li, 2013; Akbalik et al., 2017; Phouratsamay, 2017). We consider a Full-Truck-Load procurement cost structure which is studied by many papers in the literature (e.g. Li et al., 2004; Akbalik and Rapine, 2013, 2018). These papers consider neither the buyback contract nor the lost sales.

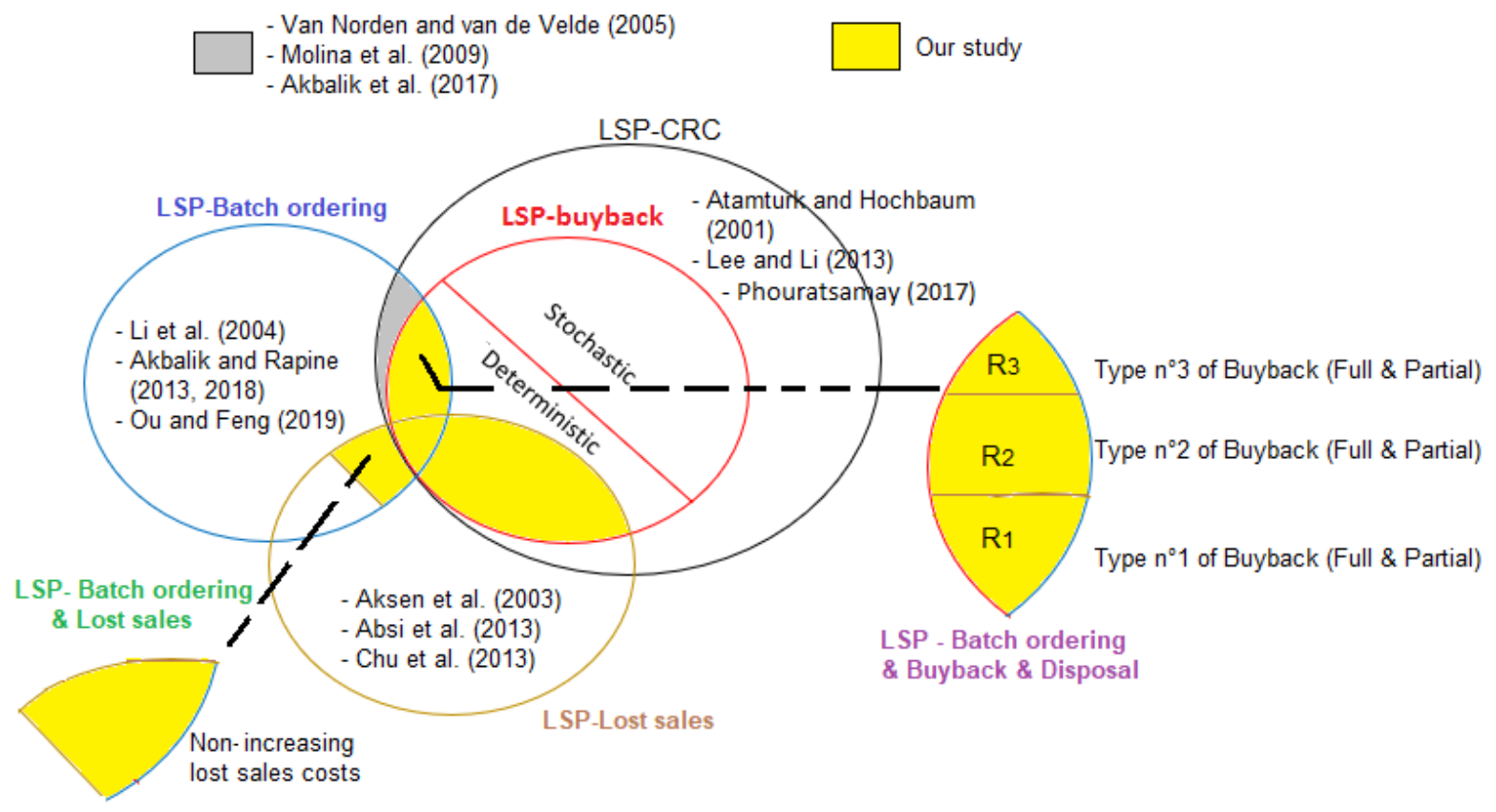

Figure 1.10: The buyback contract contributions in this study

In addition, we consider three forms of the buyback contract taken from different real-life applications. The first one is with fixed return periods $\left(R_{1}\right)$. In the form $R_{1}$, we have cyclic or acyclic return periods. The second is with a time limit on returns $\left(R_{2}\right)$, and the third form is with return possibility permitted only in procurement periods $\left(R_{3}\right)$. For each type, we study two return policies depending on the value of the maximum return percentage $\rho$ : a full return policy $(\rho=1)$ in which the retailer has the possibility to return all the unsold items, and, a partial return policy $(\rho<1)$ in which the retailer can only return a limited quantity of the 
unsold items. Hence, we add the disposal concept (the second contribution) to get rid of the units which cannot be stored nor returned to the supplier. Our problem is different from the LSP with remanufacturing, because there are no returned products from the customer to the retailer. We are interested in a buyback contract in which the retailer returns unsold goods to the supplier (disposal 1) and the remaining ones are discarded (disposal 2). Thus, at the retailer level, in a period $t$, the returned quantity is an outgoing flow and not an incoming flow as in the reverse LSP with remanufacturing. The disposal option is integrated because the products are ordered in batches. Our work looks like the LSP with all-units discount and resales of Li et al. (2012) but we cannot apply their algorithm whose running time is $O\left(T^{m+3}\right)$ because we can have a big number of breakpoints $m=+\infty$, and buyback contract constraints.

To the best of our knowledge, the batch ordering problem that is integrated with lost sales has never been addressed in the literature. There are a few papers making lost sales assumption for LSP (Aksen et al., 2003; Absi et al., 2011; Hwang et al., 2013) but without batch ordering. Thus, the third particularity of our problem is to solve this LSP both with batch ordering and non-increasing lost sales costs.

We also consider the LSP under a capacity reservation contract. So, the fourth contribution is to study this problem with time-dependent batch sizes considering three cases of reserved capacity: fixed, and (constant or time-dependent) decision variable. We propose pseudo-polynomial algorithms for some cases. The fifth contribution is to model the LSP-B under the specialized and general contracts of CRC. We prove that the LSP-B (B for Batch) under deductible reservation contract, and the LSP-B under take-or-pay contract are similar to the LSP-B under a general CRC.

\subsection{Conclusion}

In this chapter we have positioned our study in the literature by presenting the existing works in both areas: the supply chain contracts and the lot sizing problem. These two axes constitute the state of the art of our problematic. In the chapters that follow, we describe various LSP with batch ordering under the buyback contract that we have studied, modeled and solved using different approaches. We begin, in Chapter 2, by integrating the first type of buyback contract $R_{1}$ with full returns and cyclic return periods $(w, 2 w, \ldots, N w)$ into the procurement planning with the lost sales option. 


\section{Chapter 2}

\section{Lot sizing problem with batch ordering under periodic buyback contract (LSP-BR 1 with $w \geq 1$ ) and lost sales}

In this chapter, we study the deterministic single-item lot sizing problem with batch ordering under the buyback contract $R_{1}$ with cyclic returns in a system with one retailer and one supplier. We consider a fixed cost per batch replenished in addition to the classical lot sizing costs, making the procurement cost structure stepwise. We also consider lost sales option with a lost sales cost incurred for each unit of demand not satisfied. The buyback contract considered here consists in returning unused units at the end of every $w$ periods (fixed and cyclic returns), with a buyback revenue for each unit returned back. This problem is denoted by $L S P-B R_{1}$ with $w \geq 1$. We study this problem under both FTL (full truck load) cost structure and only full batch (OFB) replenishment assumption.

We first describe the general problem studied by introducing a mixed integer linear program (MILP). After that, we give dominance properties and we propose efficient and exact polynomial time algorithm for each simplified case of $L S P-B R_{1}$ with $w \geq 1$ which is known to be NP-hard in the general case. We present the test results carried out to compare the effectiveness of these resolution methods proposed for $L S P-B R_{1}$ with $w>1$. By concluding this chapter, we summarize in Table 2.8 all the algorithms that we proposed for the $L S P-B R_{1}$ with cyclic returns, with the assumptions made and the associated complexities.

The work described in this chapter is published in different conferences: ROADEF Metz (2017), see Farhat et al. (2017a), and IFAC Toulouse (2017), see Farhat et al. (2017b), for the case where we have $w=1$ with OFB/FTL cost functions and with/without lost sales, and, INCOM Bergamo (2018), see Farhat et al. (2018a), for the case where we have $w>1$ with OFB cost function. The results of this chapter are submitted to the International Journal of Production Economics and the paper is accepted, see Farhat et al. (2018b). 


\subsection{Introduction}

We are interested in a procurement problem where a retailer signs a buyback contract with a supplier who provides him the quantities in batches (e.g. pallets, containers or trucks). The buyback contract is a commitment in which the buyer has the possibility to return a certain percentage of the unused items at the end of the selling season to the supplier for a revenue less than or equal to the procurement cost. In the literature, the buyback contract is mostly used in single period planning problems occurring in the supply chains under stochastic demand. In our problem, we consider the multi-period deterministic setting and we suppose that the retailer has to return all the unused products (also called full return) to the supplier at the end of every $w$ periods. For instance, a period can represent a day, and returns can be operated only at the end of each week $(w=7)$.

This problem concerns especially the obsolete or perishable products. The shorter the product lifetime and the higher the inventory costs, the more the LSP-BR 1 with a fixed $w$ becomes interesting to solve. The determination of the parameter $w$ depends on the life of the product and the cost of carrying inventory, so it can vary over the planning horizon.

This chapter also presents two important variants of the batch replenishment: the first one is that the ordered quantity in each period $t$ is restricted to a multiple of a certain batch size $V_{t}$ (called only full batch - OFB) and the other is that the replenished batch can be incomplete (stepwise cost structure, FTL cost). We consider a general form of procurement cost structure that includes a fixed cost for each order (classical setup cost), a variable unit purchase cost, and a fixed cost per batch replenished that reflects a stepwise cost function composed of flat sections of size $V_{t}$ (see Figures 1.5 and 1.6 .

Furthermore, our study adds an important dimension where the retailer allows for lost sales on some periods over the horizon. Demand of such periods can be entirely or partially lost. According to Bijvank and Vis (2011), in case of stock-out of a specific product, the majority of the customers will not wait and will rather buy a different product or visit another store. Thus, in practical settings, the original demand can be considered to be lost, even if it is quite common to consider the backlogging of the excess demand in the classical inventory literature. Hence, the present work establishes efficient algorithms to solve an LSP with both batch ordering and lost sales, under the buyback contract, not yet addressed in the literature.

The rest of the chapter is organized as follows. Section 2.2 is dedicated to the description of the LSP-BR 1 with $w \geq 1$, and proposes a mathematical formulation by a mixed integer linear program. Section 2.3 presents LSP-BR 1 with $w \geq 1$ without lost sales, under OFB or FTL patterns. Exact polynomial time algorithms are proposed for the case with $w=1$, and followed by the general case with $w>1$. Section 2.4 deals with the $\mathrm{LSP}_{-\mathrm{BR}}$ with $w \geq 1$ under lost sales, and presents respective dynamic programming (DP) algorithms for different extensions. In Section 2.5, the computational performance of each dynamic programming algorithm is compared to that of MILP. Section 2.6 summarizes the chapter.

\subsection{Description of the $\mathrm{LSP}_{-\mathrm{BR}}$ with $w \geq 1$, hypotheses and mathematical formulation in MILP}

In the system we consider, a retailer is replenished by batches of a single-item from an external supplier, where both parties sign a buyback contract. At the beginning of each period $t$, the retailer can purchase a certain amount denoted by $x_{t}$ with a known demand $d_{t}$ over a planning horizon of $T$ periods. In real life applications, the batches can correspond to pallets, containers 
or trucks. Hence, we introduce the variable $A_{t}$ which represents the number of batches ordered in period $t$. A batch can hold up to $V_{t}$ units in period $t$. The retailer accepts to pay a cost of $a_{t}$ for each batch replenished from the supplier in addition to a unit procurement cost $p_{t}$. If there is an order in period $t$, a fixed ordering cost $f_{t}$ is incurred. An inventory holding cost $h_{t}$ is paid for each unit remaining in stock at the end of period $t$. We denote by $s_{t}$ the stock level at the end of period $t$. Without loss of generality, we assume that the initial inventory at the beginning of period 1 is zero. Otherwise, the positive quantity in stock can be retrieved from the demands of the first periods, till obtaining null entering inventory.

In the buyback contract we consider, the return of unused products is made at the end of every $w$ periods with $w \geq 1$. In classical settings of the literature, the quantity to be returned to the supplier is at the end of the selling season in a stochastic single-period problem. In general, under such contracts, the supplier allows the retailer to return a maximum of $\rho x$ units, with $x$ being the quantity purchased at a price $p$ per unit and $\rho$ being the maximum return percentage $(0 \leq \rho \leq 1)$, at a certain return revenue $p^{b}$ with $0 \leq p^{b} \leq p$. We assume a full return $(\rho=1)$ buyback contract with partial refund $\left(p^{b}<p\right)$. Our buyback contract involves $q_{t}$ and $p_{t}^{b}$, with $q_{t}$ being the returned quantity at the end of period $t$ which is a decision variable and $p_{t}^{b}$ being the unit return revenue in period $t$ which is a given parameter.

In addition to the quantities and costs mentioned above, we introduce two notations of lost sales $\left(L_{t}, l_{t}\right)$, with $L_{t}$ being the lost sales quantity in period $t$ and $l_{t}$ being the unit lost sales cost in period $t$. We consider the assumption of Aksen et al. (2003) which asserts that the gross marginal profit $\left(l_{t}-p_{t}\right)$ is nonnegative for each $t=1, \ldots, T$, but at the same time we have to take into account the batch ordering cost. Hence, we can make the following assumption without loss of generality: $l_{t} V_{t} \geq p_{t} V_{t}+a_{t}, \forall t=1, \ldots, T$, which means that losing an entire batch incurs a cost greater than or equal to procuring it. If we have $l_{t} V_{t}<p_{t} V_{t}+a_{t}$ in a period $t$, the retailer never orders in that period. Table 2.1 summarizes the notations that are used.

Table 2.1: Notations for the general model of the LSP-BR 1 with $w \geq 1$

\begin{tabular}{ll} 
Parameters & \\
\hline$T$ & length of the horizon \\
$w$ & periodicity of the return periods with $1 \leq w \leq T$ \\
$p_{t}$ & unit procurement cost (variable cost) in period $t$ \\
$f_{t}$ & fixed ordering cost (setup cost) in period $t$ \\
$a_{t}$ & fixed cost per batch replenished in period $t$ \\
$h_{t}$ & inventory holding cost per unit at the end of period $t$ \\
$p_{t}^{b}$ & unit return revenue in period $t$ \\
$l_{t}$ & unit lost sales cost in period $t$ \\
$d_{t}$ & demand in period $t$ \\
$V_{t}$ & batch size in period $t$ \\
& \\
Decision variables & \\
\hline$x_{t}$ & amount of procurement in period $t$ \\
$y_{t}$ & 1 if a procurement takes place in period $t$ \\
$A_{t}$ & 0 otherwise \\
$s_{t}$ & number of batches ordered in period $t$ \\
$q_{t}$ & stock level (quantity remaining in stock) at the end of period $t$ \\
$L_{t}$ & returned quantity of unused products at the end of period $t$
\end{tabular}

In this chapter, we study two main cases: $\mathrm{LSP} \mathrm{BR}_{1}$ with $w \geq 1$ without lost sales, which means that demands are to be entirely satisfied, and LSP-BR 1 with $w \geq 1$ and lost sales. 
Thereafter, for each of these two problems, we study the following sub-cases:

- Sub-case 1 noted by LSP-B ${ }_{O F B} \mathrm{R}_{1}$ : The supplier delivers the items only in full batches, which means that the ordered quantity in every period of the planning horizon should be expressed as a multiple of the batch sizes.

- Sub-case 2 noted by $L S P-B_{F T L} R_{1}$ : Without any restriction on the batch status, which means an FTL cost structure. In a period $t$, the batches replenished can be fractional.

For each case and sub-case, we consider two return policies. The first one is the LSP-BR with $w=1$ which means that the product return is made at the end of each period $t$. For instance, in the Hungarian market, the retailers return the unsold books $(\rho=1)$ at the end of each month without any or with a small charge (Dobos and Wimmer, 2010). The second one is the LSP-BR with $_{1}>1$, which means that the return is at the end of every $w$ periods with $w>1$. For example, at the end of summer, Procter \& Gamble, an American manufacturer of hygiene and beauty products, refunds its retailers the unsold inventory value of Bain-de-soleil $(\rho=1)$ which is a sun care product line (Padmanabhan and Png, 1997). In total, we study 8 problems, as shown in Table 2.8

\section{Assumptions}

The LSP-BR 1 with $w \geq 1$, with and without lost sales have one general assumption as follows:

- $\quad \rho=1$ (full return).

In addition, the LSP-BR 1 with $w \geq 1$ and lost sales has the following assumption, without loss of generality:

- $l_{t} V_{t} \geq p_{t} V_{t}+a_{t}, \forall \mathrm{t}=1, \ldots, T$.

Moreover, for the LSP-BR 1 with $w=1$, we consider the following assumptions:

- Batches with time-dependent sizes $\left(V_{t}\right)$.

- $p_{t}^{b}<p_{t}, \forall \mathrm{t}=1, \ldots, T$, this is a criterion of the buyback contract.

In contrast, for $\mathrm{LSP}_{-} \mathrm{BR}_{1}$ with $w>1$, we assume:

- Constant batch size $(V)$.

- $p_{i w}^{b}<p_{t}, \forall t \in\{(i-1) w+1, \ldots, i w\}$ and $\forall i \in\left\{1, \ldots, \frac{T}{w}\right\}$ with $i \in \mathbb{N}^{*}$.

- Non-increasing (NI) lost sales costs are considered to fit the realistic conditions of perishable or obsolete products.

Indeed, in general, the lost sales incur a cost corresponding to the lost revenue (selling price) and another related to the loss of customer goodwill at a later time. In our work, we assume that the lost sales cost is equal to the selling price, as the case of most research papers because estimating the future impact on customers is typically hard. Usually, solving the LSP with the goodwill loss concept in addition to revenue loss is achieved using heuristic methods, whereas in the present work, our objective is to propose exact methods. The reader can refer to Chen and Zhang (2017) to see that the lost demand due to the cost of customer goodwill has not received much attention in the academic literature. 
It is well known that the obsolete product loses its value through time because of rapid changes of technology, and this usually results in a decrease in their selling price. For example, the mobile phone loses a part of its primary selling price when there is an introduction of a new product. For a perishable product, the retailer can offer selling price discount over time (Goyal and Giri, 2001). All those observations allow us to consider NI lost sales costs in our assumptions.

We model LSP-BR 1 with $w \geq 1$ and lost sales, FTL cost structure (LSP-B ${ }_{L S, F T L} \mathrm{R}_{1}$ with $w \geq 1$ ) as a mixed integer linear program (MILP) taking into account all the features of the problem. The latter is the most general case of our problem. The number of $w$-time intervals in the planning horizon $1 . . T$ is denoted by $N$, with $N=\left\lfloor\frac{T}{w}\right\rfloor$ and $1 \leq N \leq \frac{T}{2}$. The problem with the fractional $\frac{T}{w}$ is solved as the one with the integer $\frac{T}{w}$. The only difference with the fractional $\frac{T}{w}$ is the last interval being smaller than the others. We consider that at the end of each period $i w$ with $1 \leq i \leq N$, the retailer returns the quantity $q_{i w}$ to the supplier. The total cost of the related problem depends on the decision of when and how much quantities to order, to return to the supplier, to lose and to store. The MILP for the $\operatorname{LSP}_{-B_{L S, F T L}} \mathrm{R}_{1}$ with $w \geq 1$, defined by (2.1)-(2.10) and denoted as $P_{B R_{1}}$ is given below, whose objective is to minimize the total cost $\mathscr{C}$ of setup, procurement, inventory and shortage taking into account return revenues.

$$
\mathrm{P}_{B R_{1}}\left\{\begin{array}{lll}
\min \mathscr{C}=\sum_{t=1}^{T}\left(f_{t} y_{t}+\left(p_{t} x_{t}+a_{t} A_{t}\right)+h_{t} s_{t}+l_{t} L_{t}-p_{t}^{b} q_{t}\right) \\
\text { s.t. } \quad x_{t}+s_{t-1}=d_{t}-L_{t}+s_{t}+q_{t} & \forall t=1, \ldots, T \\
& q_{t}=0 & \\
& & t=1, \ldots, T \\
& & i=1, \ldots, N \\
& s_{i w}=0 & \forall i=1, \ldots, N \\
s_{0}=0 & \\
L_{t} \leq d_{t} & \forall t=1, \ldots, T \\
& x_{t} \leq\left\lceil\frac{\sum_{j=t}^{T} d_{j}}{V_{t}}\right\rceil V_{t} y_{t} & \forall t=1, \ldots, T \\
& x_{t} \leq A_{t} V_{t} & \forall t=1, \ldots, T \\
& x_{t}, s_{t}, q_{t}, L_{t} \in \mathbb{R}_{+} & \forall t=1, \ldots, T \\
& A_{t} \in \mathbb{N}, y_{t} \in\{0,1\} & \forall t=1, \ldots, T
\end{array}\right.
$$

Constraints (2.2) are the balancing constraints linking inventory, ordering quantity, returned quantity, demand and lost sales quantities for all periods. In Constraints (2.3), no returns of unsold goods are permitted for the periods different from $i w$. In every $w$ periods, we do not talk about stored goods but rather about returned goods, that's why Constraints (2.4) ensure that the stock levels are equal to zero for those periods. Therefore, our problem is decomposed into $N$ independent problems with these constraints. Constraint (2.5) provides the initial inventory value as zero owing to problem simplification. Constraints (2.6) make sure that any amount of unmet demand $L_{t}$ in period $t$ cannot exceed the demand $d_{t}$ of that period. Constraints (2.7) ensure the setup variable generation in which the procurement variable cannot exceed the maximum quantity purchased from that period till $T$. Constraints (2.8) are for the batch replenishment which means that there are sufficient pallets containing the product quantity in each period. The remaining constraints (2.9) and (2.10) are nonnegativity, integrality and binary constraints on the decision variables. 


\section{$2.3 \quad$ LSP-BR $_{1}$ without lost sales}

The unused items are returned to the supplier, without lost sales, at the end of each period $i w$ with $i=1, \ldots, N, N=\frac{T}{w}$, and $1 \leq w \leq T$. We suppose, without loss of generality that $N$ is integer, because if it is not, this problem is solved in the same way as the one with $\frac{T}{w} \in \mathbb{N}$. Therefore, there are $N$ independent problems to solve related to each subproblem $\llbracket(i-1) w+1, i w \rrbracket$ $\forall i=1, \ldots, N$ (see Figure 2.1). We optimize each subproblem as $\llbracket 1, w \rrbracket$. The total cost of the initial problem is computed as the sum of these $N$ independent problems' costs.

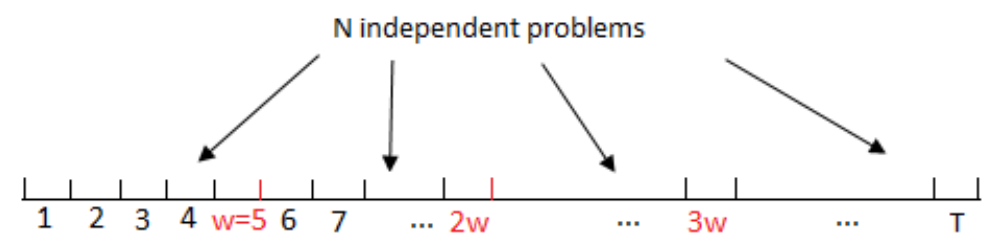

Figure 2.1: The $N$ intervals of our problem (illustration for $w=5$ )

For the return policy $w=1$, the left-over items are sold to the supplier at the end of each period $t$, thus, there is no remaining stock at retailer level over the whole horizon $\left(s_{t}=0, t=\right.$ $1, \ldots, T)$. Note that without any stock linking the periods, we can no more call this problem as "lot sizing". Indeed, there are $T$ independent problems to solve, one separate problem for each period $t, t=1, \ldots, T$ to optimize. Thus, in the following, we denote this latter as single period problem, $S P-B R_{1}$, instead of LSP-BR ${ }_{1}$ with $w=1$. Furthermore, we consider in this return policy that we have a full return with time-dependent batch sizes, and a unit return revenue lower than a unit procurement cost in every period.

For the return policy $w>1$, we have $N$ independent problems to solve with $1 \leq N \leq \frac{T}{2}$. Besides, we restrict ourselves to the case with stationary batch size in order to propose exact polynomial time algorithms. Note that Akbalik and Rapine (2013) show that the uncapacitated batch lot sizing problem with time-dependent batch sizes is NP-hard if any one of the cost parameters (setup, fixed cost per batch, unit procurement cost or unit inventory holding cost) is allowed to be time-dependent. Thus, our problem is also NP-hard for the case with timedependent batch sizes. Besides, in practical cases, the batch sizes do not really vary from one period to another. In the subproblem $\llbracket 1, w \rrbracket$, we also consider that the return revenue $p_{w}^{b}$ in period $w$ is strictly less than all the procurement costs $p_{t} \forall t \in\{1, \ldots, w\}$ to protect the supplier. We also consider the full return.

We study the four sub-cases, namely: $S P-B R_{1}$ with only full batches, LSP-BR $_{1}$ with only

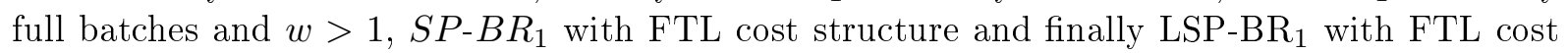
structure and $w>1$.

\subsubsection{Single Period problem-BR $\mathbf{R}_{1}$ with only full batches (SP-B $\mathrm{B}_{O F B} \mathbf{R}_{1}$ )}

In $P_{B R_{1}}$, we set $w=1, l_{t}=+\infty, \forall t=1, \ldots, T$. Thus, we have to return unused products at the end of each ordering period. Besides, we replace Constraints (2.3) with $q_{t} \geq 0, \forall t=1, \ldots, T$, Constraints (2.4) with $s_{t}=0, \forall t=1, \ldots, T$ and Constraints (2.8) with $x_{t}=A_{t} V_{t}, \forall t=1, \ldots, T$. Consequently, we obtain the $S P-B_{O F B} R_{1}$. We state an important dominance property of this problem. 
Property 2.1. An optimal plan $\pi^{*}$ orders the positive quantity $x_{t}^{*}=\left\lceil\frac{d_{t}}{V_{t}}\right\rceil V_{t}$ in every period $t \in\{1, \ldots, T\}$.

Proof. The procurement quantity in period $t$ is provided in only full batches in a feasible plan to satisfy the demand $d_{t}$. By our model assumption, the gross marginal procurement cost after returning the unused products $\left(p_{t}-p_{t}^{b}\right)$ is positive for each period. Hence, all these features give an optimal procurement quantity of $\left\lceil\frac{d_{t}}{V_{t}}\right\rceil V_{t}$.

In the $S P-B_{O F B} R_{1}$, the retailer returns the surplus $q_{t}=\left\lceil\frac{d_{t}}{V_{t}}\right\rceil V_{t}-d_{t}$ to the supplier at the end of $t$. It is then easy to compute the total ordering cost without any optimization process as follows:

$$
\mathscr{C}^{*}{ }_{B R_{1}, O F B, w=1}=\sum_{t=1}^{T}\left(f_{t}\left\lceil\frac{d_{t}}{d_{t}+1}\right\rceil+p_{t}\left\lceil\frac{d_{t}}{V_{t}}\right\rceil V_{t}+a_{t}\left\lceil\frac{d_{t}}{V_{t}}\right\rceil-p_{t}^{b}\left(\left\lceil\frac{d_{t}}{V_{t}}\right\rceil V_{t}-d_{t}\right)\right)
$$

This cost is constant since it depends only on known cost parameters and $d_{t}, V_{t}$ values. It is easy to see that a linear time algorithm in $O(T)$ solves this planning problem to optimality. This consists in determining $x_{t}^{*}$ by scanning each $t$ in $\{1, \ldots, T\}$ to have $x_{t}^{*}=\left\lceil\frac{d_{t}}{V_{t}}\right\rceil V_{t}$.

\subsubsection{LSP-BR $_{1}$ with only full batches and $w>1\left(\mathbf{L S P}-\mathbf{B}_{O F B} \mathbf{R}_{1}\right.$ with $\left.w>1\right)$}

In $P_{B R_{1}}$, we assume $l_{t}=\infty, V_{t}=V, \forall t=1, \ldots, T$, and we modify Constraints (2.8) by $x_{t}=A_{t} V, \forall t=1, \ldots, T$ to obtain the LSP-B ${ }_{O F B} \mathrm{R}_{1}$ with $w>1$. Recall that the $N$ independent problems are separately solved in order to compute the total optimal cost of LSP-B ${ }_{O F B} \mathrm{R}_{1}$ with $w>1$ by summing up individual costs of subproblems $\llbracket(i-1) w+1, i w \rrbracket, i=1, \ldots, N$. In what follows, we only consider how to solve the subproblem $\llbracket 1, w \rrbracket$ which can be applied to all subproblems. Li et al. (2004) study the LSP with batch ordering, time varying cost parameters and backlogging where the production quantity in each period is limited to a multiple of constant batch size $V$. The authors propose an algorithm that runs in $\mathrm{O}\left(T^{2}\right)$ time which is then lowered to $O(T \log (T))$ time through Monge matrices. In $\mathrm{LSP}-\mathrm{B}_{O F B} \mathrm{R}_{1}$ with $w>1$, the backlog is not allowed and the buyback contract is considered. Notice that we use a solution algorithm very close to that proposed by Li et al. (2004) with additional return quantities.

The idea of the algorithm is to detect the replenishment periods for determining the optimal total cost of $\llbracket 1, w \rrbracket$. Between two replenishment periods $i$ and $j$, we have to satisfy the demands without backlogging nor lost sales taking into consideration the stock levels at the beginning of period $i$ and at the end of period $j$. We define the following notations, similar to the ones in $\mathrm{Li}$ et al. (2004) which will be used in our approach:

$D_{i, j}$ : Cumulative demand from period $i$ to period $j$ if $i \leq j$.

$$
D_{i, j}=\left\{\begin{array}{ll}
\sum_{k=i}^{j} d_{k} & i \leq j \\
0 & i>j
\end{array} \quad \forall i, \forall j \in\{1, \ldots, w\}\right.
$$

$R_{j}$ : Minimum ending inventory level of period $j$ if we order in period 1 the minimal number of batches $\left\lceil\frac{D_{1, j}}{V}\right\rceil$ to cover the demand from period 1 to period $j$. For every $j$, we have $0 \leq R_{j} \leq V$.

$$
\begin{aligned}
& R_{0}=0 \\
& R_{j}=\left\lceil\frac{D_{1, j}}{V}\right\rceil V-D_{1, j} \quad \forall j \in\{1, \ldots, w\}
\end{aligned}
$$


For $j=1,2, \ldots, w-1$, the ending inventory level of period $j$ has the following form: $s_{j}=R_{j}+\alpha_{j} V$ with $\alpha_{j} \in \mathbb{N}$.

We now cite two suitable dominance properties for this problem.

Property 2.2. There exists an optimal solution such that, for any replenishment period $j$, the ending inventory value of period $j-1$ is equal to $R_{j-1}$ with $s_{j-1}=R_{j-1}$ and $\alpha_{j-1}=0$, $\forall j=1, \ldots, T$.

Property 2.2. is a special case of Property 2 stated in Li et al. (2004).

Proof. If $j$ is a replenishment period, then the procurement cost of one batch at $j\left(p_{j} V+a_{j}\right)$ is less than the one at a previous ordering period $t\left(p_{t} V+a_{t}\right)$ plus the inventory cost of one batch from the beginning of period $t$ to the beginning of period $j\left(\sum_{k=t}^{j-1} h_{k} V\right)$. Hence, the retailer should not procure extra batches before period $j$ (for example period $t$ ) in order to satisfy the demands after $j$ (j included). The quantity $\sum_{t=1}^{j-1} x_{t}$ is ordered in only full batches to satisfy only the demands $d_{1}, d_{2}, \ldots, d_{j-1}$ without backlogging nor lost sales. Thus, we obtain $\sum_{t=1}^{j-1} x_{t}=\left\lceil\frac{D_{1, j-1}}{V}\right\rceil V$ and $s_{j-1}=\left\lceil\frac{D_{1, j-1}}{V}\right\rceil V-D_{1, j-1}=R_{j-1}$.

Property 2.3. In any optimal policy, the returned quantity of unused products at the end of period $w$ to the supplier is $q_{w}=R_{w}$.

Proof. The objective of LSP-B $\mathrm{B}_{O F} \mathrm{R}_{1}$ with $w>1$ is the minimization of the total cost of setup, procurement, inventory and shortage taking into account return revenues such as $p_{w}^{b}<p_{t}$, $\forall t \in\{1, \ldots, w\}$. Therefore, we must acquire a minimum number of batches to satisfy the demand from period 1 to the last period $w$. It means that $\sum_{j=1}^{w} x_{j}=\left\lceil\frac{D_{1 w}}{V}\right\rceil V$. The returned quantity is obtained as follows: $q_{w}=\sum_{j=1}^{w} x_{j}-D_{1 w}$, so we have $q_{w}=R_{w}$.

We will propose an algorithm which solves the LSP-B ${ }_{O F B} \mathrm{R}_{1}$ with $w>1$ by using Properties 2.2 and 2.3. The idea is to compute the optimal cost $C_{j}$ satisfying the demand of periods $1,2, \ldots, j$, given that period $j+1$ is a replenishment period with $1 \leq j \leq w-1\left(s_{j}=R_{j}\right)$, and $j$ is a return period with $j=w\left(s_{w}=0\right.$ and $\left.q_{w}=R_{w}\right)$. An $O\left(w^{2}\right)$ time dynamic programming algorithm (see Figure 2.2 is presented as follows in order to compute the optimal total $\operatorname{cost} C_{w}$ of the subproblem $\llbracket 1, w \rrbracket$ :

Recurrence relation. For $j=1,2, \ldots, w$,

$$
C_{j}=\min _{0 \leq i<j}\left\{C_{i}+f_{i+1}+\left(p_{i+1}+\frac{a_{i+1}}{V}\right)\left(R_{j}+D_{i+1, j}-R_{i}\right)+H_{i+1, j+1}\right\}
$$

Boundary condition. $C_{0}=0$

Objective. $C_{w}$

In this relation, the periods $i+1$ and $j+1$ are consecutive ordering periods. The number of batches acquired between periods $i+1$ and $j$ can be easily computed because we know the ending inventory level of period $i$ which is equal to $R_{i}$, and the one of period $j$ which is maintained at $R_{j}$. We add to the ordering cost at period $i+1$, the total holding cost $H_{i+1, j+1}$ from the end of period $i+1$ up to the beginning of period $j+1$ knowing that there is no replenishment between period $i+2$ and period $j$, and for $0 \leq i<j \leq w-1$ we have $s_{j}=R_{j}$ and $s_{w}=0$, and for $j=w$ 


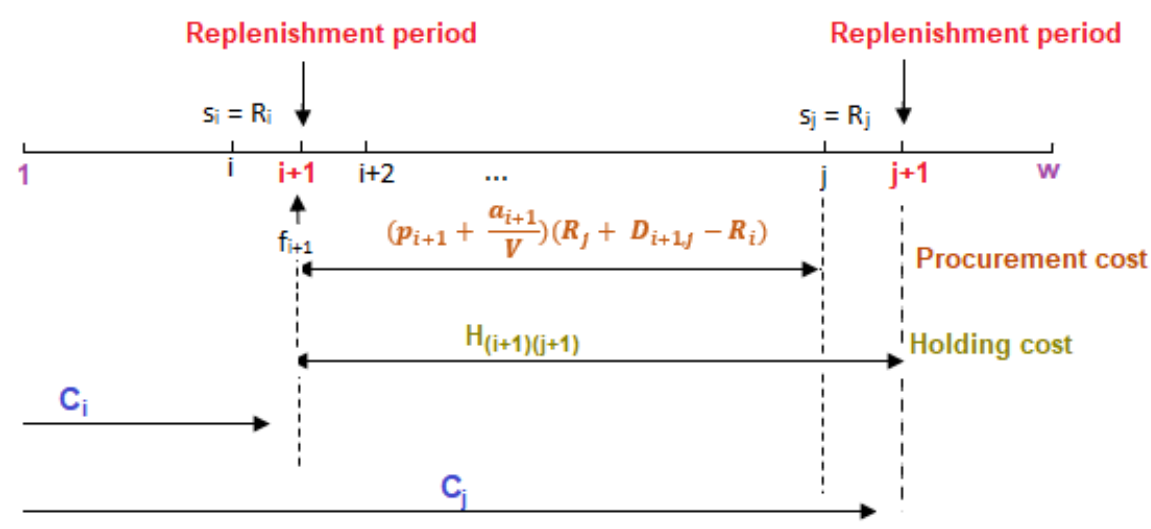

Figure 2.2: Recurrence relation of LSP-B $\mathrm{B}_{O F B} \mathrm{R}_{1}$ with $w>1$

we have $q_{w}=R_{w}$. All $H_{i+1, j+1}$ values can be obtained recursively in $O\left(w^{2}\right)$ time as follows with $\left|H_{w+1, w+1}\right|$ representing the return revenues in period $w$ :

$$
\begin{cases}H_{i+1, j+1}=H_{i+2, j+1}+h_{i+1}\left(D_{i+2, j}+R_{j}\right) & \text { if } 0 \leq i<j \leq w \\ H_{j+1, j+1}=0 & \text { if } 1 \leq j \leq w-1 \\ H_{w+1, w+1}=-p_{w}^{b} R_{w} & \text { if } j=w\end{cases}
$$

Since there are $\frac{T}{w}$ independent problems, the complexity of the whole LSP-B ${ }_{O F B} \mathrm{R}_{1}$ with $w>1$ is computed in $O(T w)$ time with $2 \leq w \leq T$ which is less than the $O\left(T^{2}\right)$ time algorithm of $\mathrm{Li}$ et al. (2004) because of the integration of buyback contract into LSP. Now, if we use the Monge matrices proposed by $\mathrm{Li}$ et al. (2004), which is very hard to apply, we can solve LSP-B ${ }_{O F B} \mathrm{R}_{1}$ with $w>1$ in $O(T \log w)$ time.

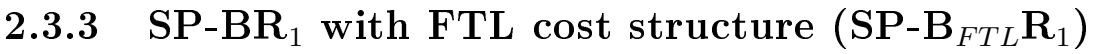

Compared to the $S P-B_{O F B} R_{1}$, we only omit the OFB assumption. We present an important optimality property of this problem.

Property 2.4. The optimal ordered quantity $x_{t}^{*}$ is exactly the demand $d_{t}$ in every period $t$.

Proof. The demand $d_{t}$ should be entirely satisfied and we have the buyback assumption $p_{t}>p_{t}^{b}$ which means that, in an optimal policy one will not order a unit uselessly to turn it back again to the supplier. Then, the solution $x_{t}^{*}$ can only be the demand $d_{t}$.

In the $S P-B_{F T L} R_{1}$, there is, thus, never a surplus at the end of return periods, which means that $q_{t}=0, \forall t=1, \ldots, T$. The total ordering cost of the $S P-B_{F T L} R_{1}\left(\mathscr{C}^{*}{ }_{B R_{1}, F T L, w=1}\right)$ is a constant, which is given as follows:

$$
\mathscr{C}^{*}{ }_{B R_{1}, F T L, w=1}=\sum_{t=1}^{T}\left(f_{t}\left\lceil\frac{d_{t}}{d_{t}+1}\right\rceil+p_{t} d_{t}+a_{t}\left\lceil\frac{d_{t}}{V_{t}}\right\rceil\right)
$$

One can obtain the optimal plan using a linear time algorithm by assigning to $x_{t}^{*}$ for each $t$ in $\{1, \ldots, T\}$ the demand $d_{t}$. 


\subsubsection{LSP-BR $_{1}$ with FTL cost structure and $w>1\left(\right.$ LSP-B $_{F T L} \mathbf{R}_{1}$ with $\left.w>1\right)$}

The LSP-B $\mathrm{B}_{F T L} \mathrm{R}_{1}$ with $w>1$ is the general model of $P_{B R_{1}}$ with constant batch size and without lost sales. We again solve each subproblem $\llbracket(i-1) w+1, i w \rrbracket$, for all $i \in\{1, \ldots, N\}$ as previously. We assume a linear full-truck-load (FTL) cost function with linear variable procurement costs. Li et al. (2004) develop an $\mathrm{O}\left(T^{3} \log T\right)$ time algorithm for the dynamic lot sizing with fractional batch ordering (LSP-B) and backlogging. The authors assume non-decreasing concave holding, backlogging, and Truck-Load (TL) freight cost functions and linear variable procurement costs. Akbalik and Rapine (2018) study a similar LSP-B but without backlogging. They consider linear holding costs, but a TL cost function with concave variable costs. Thereafter, the authors propose an $\mathrm{O}\left(T^{4}\right)$ time algorithm for LSP-B without backlogging, but with concave procurement costs being more general than that considered that of Li et al. (2004).

We decided to adapt the idea of the Akbalik and Rapine's (2018) algorithm to our subproblem $\llbracket 1, w \rrbracket$ with buyback contract, because it is much simpler to implement and to understand. It will also be used in Section 2.4.4 as one of the solutions of our problem with lost sales and batch ordering. As defined in the literature, a period $i$ is a regeneration period if it has a null entering stock level $\left(s_{i-1}=0\right)$. Furthermore, $(u, v)$ is called a subplan if periods $u$ and $v$ are consecutive regeneration periods such that $u<v$ and for each period $t=u, \ldots, v-2$, we have $s_{t}>0$. The idea of the algorithm is to compute the optimal cost $\mathscr{C}(u, v)$ of each possible subplan $(u, v)$ to satisfy the demand in periods $u, u+1, \ldots, v-1$, such that $1 \leq u<v \leq w+1$ and finally to compute the total optimal cost using a Shortest Path (SP) algorithm. Define $C_{v-1}$ as the optimal total cost to satisfy the demand in periods $1,2, \ldots, v-1$ given that period $v$ is a regeneration period $(1 \leq v \leq w+1)$. The following $\mathrm{SP}$ algorithm computes the total cost $C_{w}$ :

$$
\text { For } v=2,3, \ldots, w+1, \quad C_{v-1}=\min _{1 \leq u<v}\left\{C_{u-1}+\mathscr{C}(u, v)\right\} \quad \text { such that } C_{0}=0
$$

The running time of the above $\mathrm{SP}$ algorithm is $O\left(w^{2}\right)$ if all the $\mathscr{C}(u, v)$ values have been predetermined. Akbalik and Rapine (2018) show that finding the minimal cost value of a subplan $(u, v)$ is performed in time complexity $O\left(w^{2}\right)$. This implies that the subproblem $\llbracket 1, w \rrbracket$ is solvable in $O\left(w^{4}\right)$ time and LSP-B ${ }_{F T L} \mathrm{R}_{1}$ with $w>1$ in $O\left(T w^{3}\right)$ time.

In each subplan $(u, v), t$ and $r$ are two ordering periods such that $u \leq t<r \leq v$. If there is at most one ordering period between $t$ and $r$, then the set $\{t, t+1, \ldots, r-1\}$ is called a replenishment cycle. We denote by $[t, r]^{u, v}$ such a cycle. The calculation of $\mathscr{C}(u, v)$ requires to decompose the subplan $(u, v)$ into different forms of replenishment cycles $[t, r]^{u, v}$. Akbalik and Rapine (2018) (pages 9-11, Appendix A) describe how the optimal cost $\mathscr{L}[t, r]^{u, v}$ of each replenishment cycle $[t, r]^{u, v}$ such that $t$ and $r$ are full batch ordering periods can be computed in constant time. In our study, we explain how to compute the following costs by presenting a numerical example:

- The optimal cost $\mathscr{L}[u, t]^{u, v}$ such that $u$ is a fractional batch ordering period with $0<$ $x_{u}<V, t$ is a full batch ordering period and $u$ and $t$ are consecutive replenishment periods which means that no other ordering period exists between $u$ and $t$.

- The optimal cost $\mathscr{L}[t, r]^{u, v}$ such that $t$ is a fractional batch ordering period with $x_{t} \geq V$, $r$ is a full batch ordering period and $t$ and $r$ are consecutive replenishment periods.

- The optimal cost $\mathscr{C}(u, v)$. 


\section{Definitions and properties}

For the LSP-B ${ }_{F T L} \mathrm{R}_{1}$ with $w>1$, before defining the dominance properties related to batch ordering, we present the major property related to the buyback contract.

Property 2.5. The quantity returned to the supplier at the end of period $w$ is null $\left(q_{w}^{*}=0\right)$.

Proof. Suppose we have an optimal policy $\pi^{\prime}$ in which we return a positive quantity $q_{w}^{\prime}>0$ in period $w$. In the subproblem $\llbracket 1, w \rrbracket$, we consider that there are $n$ replenishment periods $t_{1}, t_{2}, \ldots, t_{n-1}, t_{n}$ with $1 \leq t_{1} \leq t_{2} \leq \cdots \leq t_{n-1} \leq t_{n} \leq w$.

We define $x_{t_{n}}^{\prime}$ any positive quantity purchased in the last ordering period $t_{n}$. Let $\chi^{\left(t_{n}\right)}=$ $\min \left\{x_{t_{n}}^{\prime}, q_{w}^{\prime}\right\}$.

In order to obtain another feasible policy, we will make some modifications from period $t_{n}$ till period $w$. We decrease $x_{t_{n}}^{\prime}$ to $x_{t_{n}}^{\prime}-\chi^{\left(t_{n}\right)}$ and $A_{t_{n}}^{\prime}$ to $A_{t_{n}}^{\prime}-m^{\left(t_{n}\right)}$ with $m^{\left(t_{n}\right)} \in \mathbb{N}$ and $m^{\left(t_{n}\right)}=\left\lceil\frac{x_{t_{n}}^{\prime}}{V}\right\rceil-\left\lceil\frac{x_{t_{n}}^{\prime}-\chi^{\left(t_{n}\right)}}{V}\right\rceil$. We also decrease the inventory levels $s_{g}^{\prime}$ to $s_{g}^{\prime}-\chi^{\left(t_{n}\right)}$ of the periods $g=t_{n}, \ldots, w-1$, and the returned quantity $q_{w}^{\prime}$ to $q_{w}^{\prime}-\chi^{\left(t_{n}\right)}$. This modified solution remains feasible, because, in $\pi^{\prime}$, the initial inventory levels of the periods $g$ between $t_{n}$ and $w-1$ are all greater than or equal to $\chi^{\left(t_{n}\right)}$. After the modification, they all remain positive or null.

This change decreases the total cost of the policy $\pi^{\prime}$ by an amount of $\left(p_{t_{n}}-p_{w}^{b}\right) \chi^{\left(t_{n}\right)}+m^{\left(t_{n}\right)} a_{t_{n}}+$ $\sum_{g=t_{n}}^{w-1} h_{g} \chi^{\left(t_{n}\right)}$, because of the buyback assumption $\left(p_{t}>p_{w}^{b} \forall t \in\{1, \ldots, w\}\right)$. We obtain a modified solution with a return quantity $q_{w}^{\prime}-\chi^{\left(t_{n}\right)}$. If $\chi^{\left(t_{n}\right)}=q_{w}^{\prime}$, then the new (modified) policy has a null return verifying Property 2.5. Otherwise $\left(\chi^{\left(t_{n}\right)}=x_{t_{n}}^{\prime}\right)$, in the new policy, the return quantity is positive but less than $q_{w}^{\prime}$ and the period $t_{n-1}$ becomes the last ordering period. In this case, we repeat the same procedure above in order to obtain a new policy with a zero return quantity and having a lower cost.

Property 2.6. (Lee et al., 2003) There is an optimal policy $\pi^{*}$, in which, there exists at most one fractional batch ordered in any of its subplans. It means that, for a subplan $(u, v)$, there is at most one fractional batch replenished among periods $u, u+1, \ldots, v-1$. In Figure 2.3. we suppose that $D_{u, v-1}-\left\lfloor\frac{D_{u, v-1}}{V}\right\rfloor V>0$. The fractional batch is procured either with other full batches or alone.
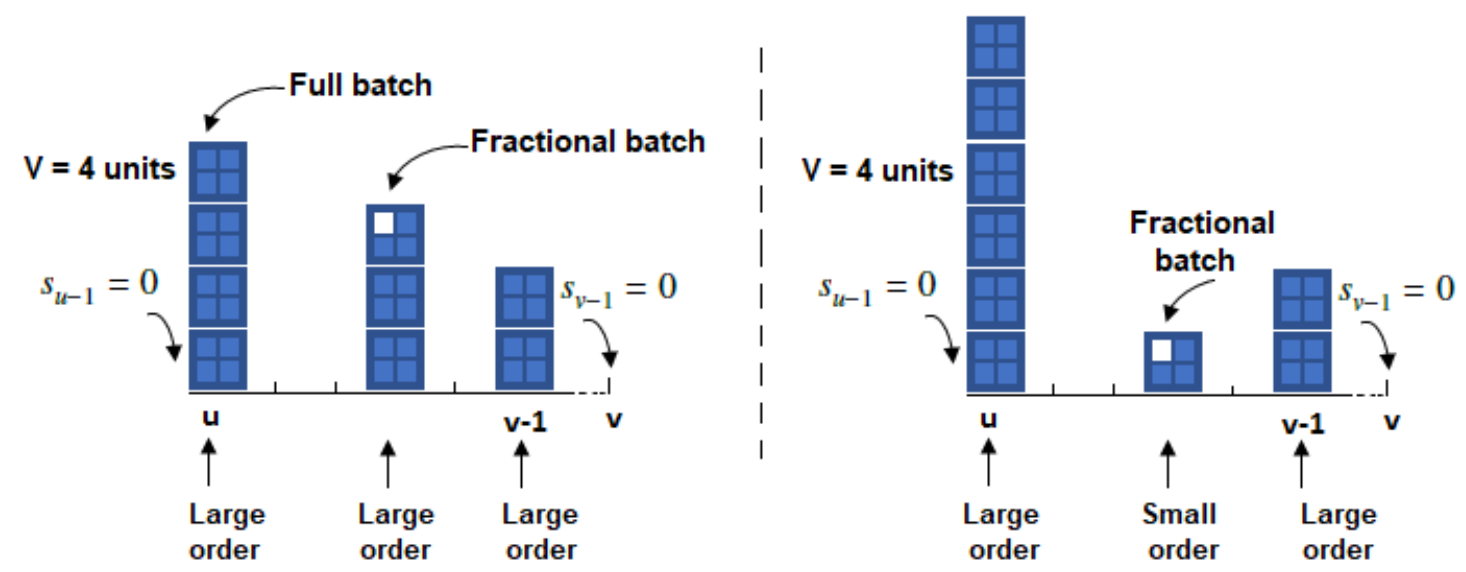

Figure 2.3: Possibilities of optimal planning of $(u, v)$ in $\operatorname{LSP}_{-} \mathrm{B}_{F T L} \mathrm{R}_{1}$ with $w>1$

Note that Lee et al. (2003) are the pioneer having stated the same property for their model. 
Property 2.6 can be proven by using the proof of Property 4, in Li et al. (2004). In the following, we introduce the definition of a large ordering period which is proposed by Akbalik and Rapine (2018) that will be used in Property 2.7.

Definition 2.1. (Akbalik and Rapine, 2018, page 9) An ordering period $t$ is called a large period if the retailer procures at least one full batch (i.e. $x_{t} \geq V$ ), and is called small $\left(x_{t}<V\right)$ otherwise (see Figure 2.3).

By Property 2.6 and Definition 2.1, there is at most one small ordering period over the subplan $(u, v)$, which corresponds to the ordering of a fractional batch. Nevertheless, the period of fractional batch ordering can be also large if this fractional batch is ordered in addition to full batches. We have the following property which is used to solve the LSP-B first stated in Akbalik and Rapine (2018).

Property 2.7. (Akbalik and Rapine, 2018, page 10) Consider that the periods $t$ and $r$ are two consecutive ordering periods. In an optimal policy $\pi^{*}$, if both periods are large, then $s_{r-1}<V$. In other words, if $s_{r-1} \geq V$ then either the period $t$ is large and $r$ is small, or vice versa.

We present a different way to prove Property 2.7 than the one proposed by Akbalik and Rapine (2018) knowing that we consider unit procurement costs.

Proof. Consider an optimal policy $\pi^{\prime}$ in which for two consecutive and large ordering periods $t$ and $r$, we have $s_{r-1}^{\prime} \geq V$. The policy $\pi^{\prime}$ involves, for any period $k$ between $t$ and $r-1$, a large stock level which means that $s_{k}^{\prime} \geq V$.

If we increase $x_{t}^{\prime}$ by $V$ and decrease $x_{r}^{\prime}$ by $V$ without changing the procurement quantities of the other periods, the modified solution remains feasible because the period $r$ in policy $\pi^{\prime}$ is large. The first new total cost will be increased by an amount of

$$
\Delta^{(\prime \prime)}=\left(p_{t}+\sum_{k=t}^{r-1} h_{k}+\frac{a_{t}}{V}-p_{r}-\frac{a_{r}}{V}\right) V
$$

If we decrease $x_{t}^{\prime}$ by $\mathrm{V}$ and increase $x_{r}^{\prime}$ by $\mathrm{V}$, the modified solution keeps its feasibility because we only anticipate the production of $V$ units from the large period $t$ to $r$ with inventory levels $s_{k}^{\prime}$ being greater than or equal to $V$ for $k=t, \ldots, r-1$. However, the second new total cost will be increased by an amount of

$$
\Delta^{(\prime \prime \prime)}=\left(p_{r}+\frac{a_{r}}{V}-p_{t}-\sum_{k=t}^{r-1} h_{k}-\frac{a_{t}}{V}\right) V
$$

By optimality of the policy $\pi^{\prime}$, we have $\Delta^{(\prime \prime)} \geq 0$ and $\Delta^{\left({ }^{\prime \prime \prime}\right)} \geq 0$. Note that $\Delta^{\left({ }^{\prime \prime}\right)}=-\Delta^{\left({ }^{\prime \prime \prime}\right)}$, therefore, $\Delta^{\left({ }^{\prime \prime}\right)}=\Delta^{\left({ }^{\prime \prime \prime}\right)}=p_{t} V+\sum_{k=t}^{r-1} h_{k} V+a_{t}-p_{r} V-a_{r}=0$ which means that buying a batch in period $t$ and storing it until the period $r$ have the same cost as purchasing it in period $r$. Now, we distinguish two cases based on the quantity ordered in period $r$ :

Case 1. $x_{r}^{\prime}=\left\lfloor\frac{x_{r}^{\prime}}{V}\right\rfloor V=\left\lceil\frac{x_{r}^{\prime}}{V}\right\rceil V$, then increasing $x_{t}^{\prime}$ to $x_{t}^{\prime}+x_{r}^{\prime}$ and decreasing $x_{r}^{\prime}$ to 0 , maintain the feasibility of the solution and lower the total cost $\mathscr{C}\left(\pi^{\prime}\right)$ by $f_{r}$. Hence, the solution of the policy $\pi^{\prime}$ cannot be optimal - a contradiction.

Case 2. $x_{r}^{\prime}=\left\lfloor\frac{x_{r}^{\prime}}{V}\right\rfloor V+\epsilon$, with $\epsilon<V$ then $x_{t}^{\prime}=\left\lfloor\frac{x_{t}^{\prime}}{V}\right\rfloor V=\left\lceil\frac{x_{t}^{\prime}}{V}\right\rceil V$ according to Property 2.6. At first, we consider the alternate policy $\pi^{(1)}$ ordering $\left\lfloor\frac{x_{r}^{\prime}}{V}\right\rfloor V+\epsilon$ additional units in period $t$, and 
nothing in period $r$, that is, $x_{t}^{(1)}=x_{t}^{\prime}+\left\lfloor\frac{x_{r}^{\prime}}{V}\right\rfloor V+\epsilon$ and $x_{r}^{(1)}=0$. Policy $\pi^{(1)}$ is clearly feasible. The total cost $\mathscr{C}\left(\pi^{(1)}\right)$ is computed as a function of the total cost $\mathscr{C}\left(\pi^{\prime}\right)$, as follows:

$$
\mathscr{C}\left(\pi^{(1)}\right)=\mathscr{C}\left(\pi^{\prime}\right)-\left(f_{r}-\left(1-\frac{\epsilon}{V}\right)\left(a_{t}-a_{r}\right)\right)
$$

If $f_{r}>\left(1-\frac{\epsilon}{V}\right)\left(a_{t}-a_{r}\right)$, then $\mathscr{C}\left(\pi^{(1)}\right)<\mathscr{C}\left(\pi^{\prime}\right)$ and the solutions $x_{t}^{\prime}$ and $x_{r}^{\prime}$ of the policy $\pi^{\prime}$ cannot be optimal - a contradiction.

If $f_{r}=\left(1-\frac{\epsilon}{V}\right)\left(a_{t}-a_{r}\right)$, then $a_{t} \geq a_{r}$ and $\mathscr{C}\left(\pi^{(1)}\right)=\mathscr{C}\left(\pi^{\prime}\right)$, thus there is an another optimal policy $\left(\pi^{(1)}\right)$ that satisfies the property.

If $f_{r}<\left(1-\frac{\epsilon}{V}\right)\left(a_{t}-a_{r}\right)$, then $a_{t}>a_{r}$, and the policy $\pi^{(1)}$ can not be optimal. Secondly, we consider the alternate policy $\pi^{(2)}$, in which $\left\lfloor\frac{x_{r}^{\prime}}{V}\right\rfloor V$ additional units are ordered in period $t$, and $\left\lfloor\frac{x_{r}^{\prime}}{V}\right\rfloor$ batches less in period $\mathrm{r}$, that is, $x_{t}^{(2)}=x_{t}^{\prime}+\left\lfloor\frac{x_{r}^{\prime}}{V}\right\rfloor V$ and $x_{r}^{(2)}=\epsilon$. Policy $\pi^{(2)}$ remains feasible. The total cost $\mathscr{C}\left(\pi^{(2)}\right)$ is equal to the total cost $\mathscr{C}\left(\pi^{\prime}\right), \mathscr{C}\left(\pi^{(2)}\right)=\mathscr{C}\left(\pi^{\prime}\right)$. The optimal solution of $\pi^{\prime}$ can be modified in such a way that the period $t$ remains large but the period $r$ becomes small.

Property 2.8. If there is a small ordering period $\bar{t}$ between two large consecutive ordering periods $t$ and $r(t<\bar{t}<r)$, then $s_{r-1}<2 V$.

Proof. The same analysis of the proof of (Property 2.7, case 1) is carried out, knowing that by considering $s_{r-1}^{\prime} \geq 2 V$, we obtain $s_{i}^{\prime}>V$ for $i=t, \ldots, \bar{t}-1$ and $s_{j}^{\prime} \geq 2 V$ for $j=\bar{t}, \ldots, r-1$, and that the quantities ordered in periods $t$ and $r$ are multiples of $V$ according to Property 2.6.

\section{How to compute $\mathscr{L}[t, r]^{u, v}$ in the subplan $(u, v)$}

In a given subplan $(u, v)$, we need to compute the cost $\mathscr{L}[t, r]^{u, v}$ to satisfy the demands $d_{t}, d_{t+1}, \ldots, d_{r-1}$ of the replenishment cycle $[t, r]^{u, v}$ in different situations. Recall that, there is at most one fractional batch occurring over the time horizon $u, \ldots, v-1$, so we denote by $\bar{x}_{u, v}$ the fractional quantity ordered in the incomplete batch such that $\bar{x}_{u, v}=D_{u, v-1}-\left\lfloor\frac{D_{u, v-1}}{V}\right\rfloor V$ and by $\bar{t}_{u, v}$ the fractional ordering period such that $u \leq \bar{t}_{u, v}<v$. The period $\bar{t}_{u, v}$ can be a large or a small ordering period. Thereafter, we distinguish 3 cases according to the criterion and the position of $\bar{t}_{u, v}$ in $(u, v)$. In the first case, the subplan $(u, v)$ has a small period $\bar{t}_{u, v}$ such that $\bar{t}_{u, v}>u$. In the second case, $\bar{t}_{u, v}$ remains small but we have $\bar{t}_{u, v}=u$. In the last case, $\bar{t}_{u, v}$ is large. In each case, there are different situations. Finally, we can have 8 possible values of the cost $\mathscr{L}[t, r]^{u, v(i)}$ with $i=1, \ldots, 8$ according to different cases (see Table 2.2). Refer to Figure 2.4 in order to view the different situations of $[t, r]^{u, v}$ and $(u, v)$.

All the ending inventory levels must be calculated for the periods $u, u+1, \ldots, v-2$ because we have $s_{u-1}^{u, v}=s_{v-1}^{u, v}=0$. In order to calculate all the values of $\mathscr{L}[t, r]^{u, v(i)}$, for $1 \leq u \leq t<$ $r \leq v \leq w+1$ and $i=1, \ldots, 8$, we discern three cases composed of sub-cases depending on the nature and the place of the fractional ordering period $\bar{t}_{u, v}$. All these values can be computed in $O\left(w^{4}\right)$ time.

Case 1: $\bar{t}_{u, v}$ is small and $\bar{t}_{u, v}>u($ Akbalik and Rapine, 2018)

In this case, the periods $t$ and $r$ are large consecutive ordering periods with $t<r$. We describe how the optimal cost $\mathscr{L}[t, r]^{u, v}$ can be determined by positioning $\bar{t}_{u, v}$ before, inside or after the cycle $[t, r]^{u, v}$. We do not rewrite the explicit equations presented in Akbalik and Rapine (2018). 
Table 2.2: Costs $\mathscr{L}[t, r]^{u, v}$ in different situations

\begin{tabular}{|c|c|c|c|}
\hline $\begin{array}{l}\text { Cases } \\
(u, v)\end{array}$ & \multicolumn{2}{|l|}{ Different situations of $[t, r]^{u, v}$} & $\begin{array}{l}\text { Possible } \\
\text { costs } \\
{[t, r]^{u, v}}\end{array}$ \\
\hline \multirow{5}{*}{$\begin{array}{l}\bar{t}_{u, v} \text { is small } \\
\bar{t}_{u, v}>u\end{array}$} & \multirow{2}{*}{$\begin{array}{l}\bar{t}_{u, v}<t<r \text { with } t \text { and } r \text { are large and } \\
\text { consecutive ordering periods }\end{array}$} & $s_{t-1}^{u, v}<V$ & $\mathscr{L}[t, r]^{u, v(1)}$ \\
\hline & & $V \leq s_{t-1}^{u, v}<2 V$ & $\mathscr{L}[t, r]^{u, v(2)}$ \\
\hline & \multirow{2}{*}{$\begin{array}{l}t<\bar{t}_{u, v}<r \text { with } t \text { and } r \text { are large } \\
\text { consecutive ordering periods }\end{array}$} & $s_{r-1}^{u, v}<V$ & $\mathscr{L}[t, r]^{u, v(3)}$ \\
\hline & & $V \leq s_{r-1}^{u, v}<2 V$ & $\mathscr{L}[t, r]^{u, v(4)}$ \\
\hline & \multicolumn{2}{|c|}{$t<r<\bar{t}_{u, v}$ with $t$ and $r$ are large and consecutive ordering periods } & $\mathscr{L}[t, r]^{u, v(5)}$ \\
\hline \multirow{2}{*}{$\begin{array}{l}\bar{t}_{u, v} \text { is small } \\
\bar{t}_{u, v}=u\end{array}$} & \multicolumn{2}{|c|}{$u<t$ with 2 consecutive ordering periods $u$ and $t$, and $t$ is large } & $\mathscr{L}[u, t]^{u, v(6)}$ \\
\hline & \multicolumn{2}{|c|}{$t$ and $r$ are large and consecutive ordering periods with $u<t<r$} & $\mathscr{L}[t, r]^{u, v(7)}$ \\
\hline$\overline{\bar{t}_{u, v} \text { is large }}$ & \multicolumn{2}{|c|}{$t=\bar{t}_{u, v}$ and, $t$ and $r$ are large and consecutive ordering periods with $t<r$. } & $\mathscr{L}[t, r]^{u, v(8)}$ \\
\hline
\end{tabular}

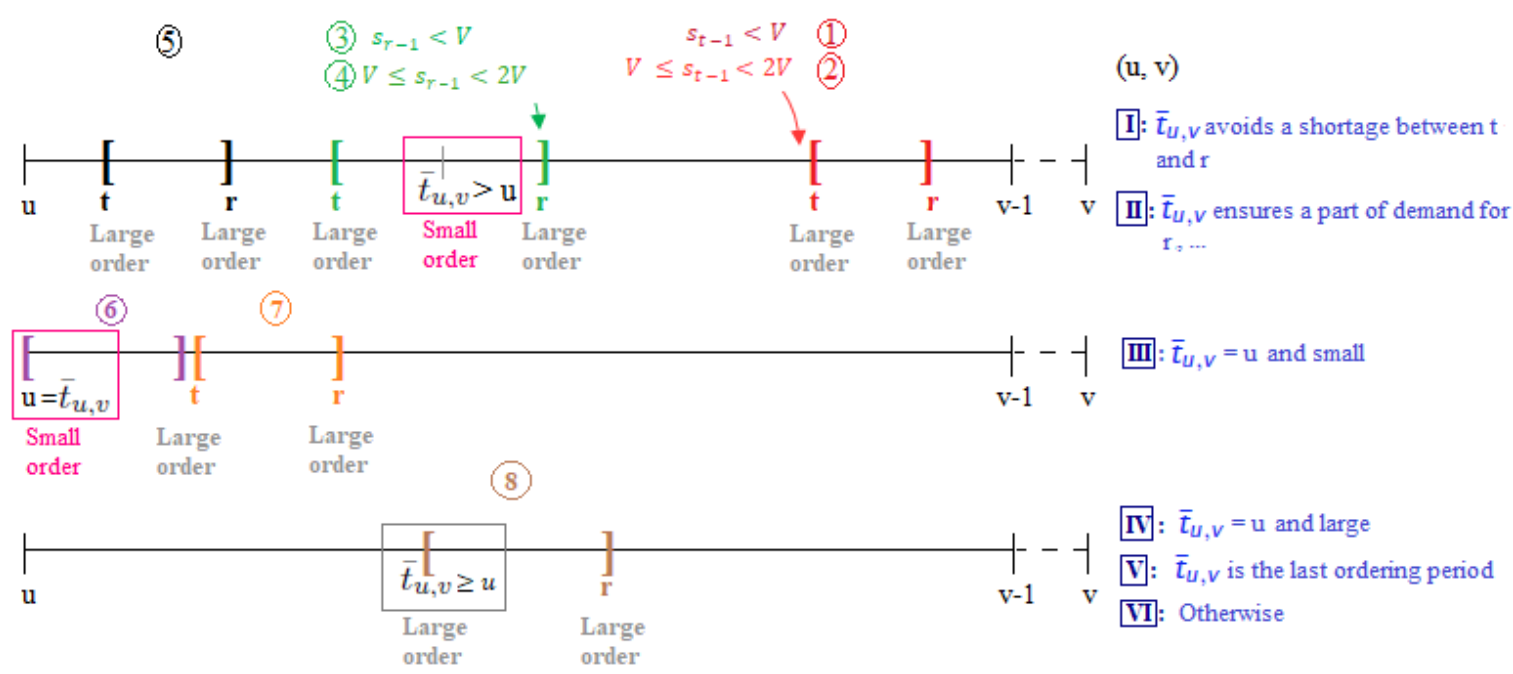

Figure 2.4: Different situations of $[t, r]^{u, v}$ and $(u, v)$

Sub-case 1.1: $\bar{t}_{u, v}<t$

In this sub-case, the large ordering periods $t$ and $r$ are consecutive. Using Property 2.7 and the fact that the quantity $\bar{x}_{u, v}$ is ordered before $t$, the ending inventory level $s_{r-1}^{u, v}$ can be written as:

$$
s_{r-1}^{u, v}= \begin{cases}\left\lceil\frac{D_{u, r-1}-\bar{x}_{u, v}}{V}\right\rceil V-\left(D_{u, r-1}-\bar{x}_{u, v}\right) & \text { if } D_{u, r-1}-\bar{x}_{u, v}>0 \\ 0 & \text { otherwise }\end{cases}
$$

The stock level $s_{t-1}^{u, v}$ depends on the nature of the preceding ordering period $k$ just before $t$.

If $k$ is large, then the Equation (2.12) can be used for $s_{t-1}^{u, v(1)}$ after replacing $r$ with $t$.

If $k$ is small $\left(k=\bar{t}_{u, v}\right)$, then there is at least a large ordering period between $u$ and $k-1$ and we obtain $s_{t-1}^{u, v(2)}<2 V$ by using Property 2.8. In this case, the stock level $s_{t-1}^{u, v(2)}$ takes two possible values. If the quantity $\bar{x}_{u, v}$ ordered in period $k$ is not used to satisfy a part of demands $d_{k}, d_{k+1}, \ldots, d_{t-1}$, then we obtain the first value $s_{t-1}^{u, v\left(2^{\prime}\right)} \geq V$ :

$$
s_{t-1}^{u, v\left(2^{\prime}\right)}= \begin{cases}V+\left\lceil\frac{D_{u, t-1}-\bar{x}_{u, v}}{V}\right\rceil V-\left(D_{u, t-1}-\bar{x}_{u, v}\right) & \text { if } D_{u, t-1}-\bar{x}_{u, v}>0 \\ +\infty & \text { otherwise }\end{cases}
$$

Otherwise, if the quantity $\bar{x}_{u, v}$ is ordered to avoid the shortage between the periods $k, k+1, \ldots, t-$ 
1 and subsequently to satisfy a demand before $t$, then we obtain the second value $s_{t-1}^{u, v}{ }^{\left(2^{\prime \prime}\right)}<V$ and $s_{t-1}^{u, v\left(2^{\prime \prime}\right)}=s_{t-1}^{u, v(1)}$. We suppose that $s_{t-1}^{u, v(2)}=s_{t-1}^{u, v\left(2^{\prime}\right)}$.

If $\bar{t}_{u, v}$ is placed before the replenishment cycle $[t, r]^{u, v}$ such that $t$ and $r$ are large and consecutive ordering periods, then we have two possible values for the cost $\mathscr{L}[t, r]^{u, v}$ according to the two values of $s_{t-1}^{u, v}$. We define $H[t, r]^{u, v}$ as the total holding cost from the end of $t$ until the beginning of $r$ if the entering stock level in period $r$ is $s_{r-1}^{u, v}$, and assuming that there is no acquisition taking place in periods $t+1, \ldots, r-1$, with $t<r$. Hence, all $H[t, r]^{u, v}$ values can be obtained recursively in $O\left(w^{4}\right)$ time by the following equations:

$$
\left\{\begin{array}{l}
H[t, r]^{u, v}=H[t+1, r]^{u, v}+h_{t}\left(D_{t+1, r-1}+s_{r-1}^{u, v}\right) \quad \text { if } t<r \\
H[t, t]^{u, v}=0
\end{array}\right.
$$

Thereafter, we give the formula of $\mathscr{L}[t, r]^{u, v(i)}$ which is defined as the cost of the replenishment cycle $[t, r]^{u, v(i)}$ with two large and consecutive ordering periods $t$ and $r$ and $\bar{t}_{u, v}<t$, for $i \in\{1,2\}$, $u \leq t<r \leq v$ and evaluated in $O\left(w^{4}\right)$ time as follows:

$$
\mathscr{L}[t, r]^{u, v(i)}= \begin{cases}f_{t}+p_{t}\left(D_{t, r-1}+s_{r, v}^{u, v}-s_{t-1}^{u, v(i)}\right) & \text { if } D_{t, r-1}+s_{r-1}^{u, v}-s_{t-1}^{u, v(i)} \geq V \\ & +a_{t}\left\lceil\frac{D_{t, r-1}+s_{r-1}^{u, v}-s_{t-1}^{u, v}(i)}{V}\right\rceil+H[t, r]^{u, v} \\ & \\ +\infty & \text { otherwise }\end{cases}
$$

Sub-case 1.2: $t<\bar{t}_{u, v}<r$

There is a small ordering period between $\mathrm{t}$ and $\mathrm{r}$, so $t<r-1$. In this sub-case, all the ordering periods before $t$ are large that is why there is only one value of $s_{t-1}^{u, v}$ obtained by the flow conservation of Constraints (2.2) as follows:

$$
s_{t-1}^{u, v}=\left\lceil\frac{D_{u, t-1}}{V}\right\rceil V-D_{u, t-1}
$$

However, the stock level at the beginning of period $r, s_{r-1}^{u, v}$ can take two values because the small period $\bar{t}_{u, v}$ is just before $r$. Thus, we obtain either $s_{r-1}^{u, v}<V$ or $V \leq s_{r-1}^{u, v}<2 V$, as discussed before.

If $s_{r-1}^{u, v}<V$, then the number of full batches ordered in period $t, x_{t}^{u, v(1)}$ and the stock $s_{t-1}^{u, v}$ cannot satisfy the sum of the demands $d_{t}, d_{t+1}, \ldots, d_{r-1}$ (with $x_{t}^{u, v(1)}+s_{t-1}^{u, v}<D_{t, r-1}$ ). Therefore, the fractional batch ordered in period $\bar{t}_{u, v}$ is used to complete the demand satisfaction in the replenishment cycle $[t, r]^{u, v}$. Therefore, we obtain:

$$
x_{t}^{u, v(1)}=\left\lfloor\frac{D_{t, r-1}-s_{t-1}^{u, v}}{V}\right\rfloor V
$$

Hence, we must, on the one hand, search for the possible positions of $\bar{t}_{u, v}$ in the cycle $[t, r]^{u, v}$ by computing the first period $\lambda[t, r]^{u, v}$ where a shortage occurs if the fractional quantity is not ordered, and, on the other hand, determine the position of $\bar{t}_{u, v}$ among its possible positions $\left\{t+1, \ldots, \lambda[t, r]^{u, v}\right\}$ by seeking the minimal ordering cost $W\left(t+1, \lambda[t, r]^{u, v}\right)$ to order $\bar{x}_{u, v}$. For the expressions of $\lambda[t, r]^{u, v}$ and $W\left(t+1, \lambda[t, r]^{u, v}\right)$, refer to Akbalik and Rapine (2018), page 10. 
Let $H^{\prime}[t, r]^{u, v}$ denote the total holding cost from the end of period $t$ until the beginning of period $r$ if the total acquisition quantity in $[t, r]^{u, v}$ is ordered in $t$ and equal to $x_{t}^{u, v(1)}$ expressed above. Then,

$$
\left\{\begin{array}{l}
H^{\prime}[t, r]^{u, v}=H^{\prime}[t+1, r]^{u, v}+h_{t}\left(x_{t}^{u, v(1)}-\left(d_{t}-s_{t-1}^{u, v}\right)\right) \text { if } t<r-1 \\
H^{\prime}[t, t]^{u, v}=0
\end{array}\right.
$$

The values of $H^{\prime}[t, r]^{u, v}$ can be obtained in $O\left(w^{4}\right)$ time. This implies that the cost $\mathscr{L}[t, r]^{u, v(3)}$ of the replenishment cycle $[t, r]^{u, v(3)}$ with two large consecutive ordering periods $t$ and $r, t<\bar{t}_{u, v}<r$ and $s_{r-1}^{u, v}<V$, for $u \leq t<r-1 \leq v-1$ is as follows:

$$
\mathscr{L}[t, r]^{u, v(3)}= \begin{cases}\left(f_{t}+\left(p_{t}+\frac{a_{t}}{V}\right) x_{t}^{u, v(1)}+H^{\prime}[t, r]^{u, v}\right. & \text { if } D_{t, r-1}-s_{t-1}^{u, v} \geq V \\ \left.+W\left(t+1, \lambda[t, r]^{u, v}\right)\right) & \& x_{t}^{u, v(1)}+s_{t-1}^{u, v}+\bar{x}_{u, v} \geq D_{t, r-1} \\ +\infty & \text { otherwise }\end{cases}
$$

The time required to precompute those costs is bounded by $O\left(w^{4}\right)$ for the subproblem $\llbracket 1, w \rrbracket$.

If $V \leq s_{r-1}^{u, v}<2 V$, then the number of full batches ordered in period $t$ satisfies all the demands $d_{t}, d_{t+1}, \ldots, d_{r-1}\left(x_{t}^{u, v(2)}+s_{t-1}^{u, v} \geq D_{t, r-1}\right)$, so we have:

$$
x_{t}^{u, v(2)}=\left\lceil\frac{D_{t, r-1}-s_{t-1}^{u, v}}{V}\right\rceil V
$$

The fractional batch ordered in period $\bar{t}_{u, v}$ is used to satisfy a demand after period $r$. Besides, it is among the set $\{t+1, \ldots, r-1\}$. Its position is determined by computing the minimum ordering cost $W(t+1, r-1)$ to order the fractional batch $\bar{x}_{u, v}$ over the set cited before.

In Equation (2.15) of $H^{\prime}[t, r]^{u, v}$, we change the quantity $x_{t}^{u, v(1)}$ to $x_{t}^{u, v(2)}$ and we obtain another total holding cost $H^{\prime \prime}[t, r]^{u, v}$ which can be predetermined in $O\left(w^{4}\right)$.

We denote by $\mathscr{L}[t, r]^{u, v(4)}$ the minimum cost of the replenishment cycle $[t, r]^{u, v(4)}$ in the case where $t$ and $r$ are two large consecutive ordering periods, $t<\bar{t}_{u, v}<r$ and $V \leq s_{r-1}^{u, v}<2 V$, for $u \leq t<r-1 \leq v-1$ :

$$
\mathscr{L}[t, r]^{u, v(4)}= \begin{cases}f_{t}+\left(p_{t}+\frac{a_{t}}{V}\right) x_{t}^{u, v(2)}+H^{\prime \prime}[t, r]^{u, v}+W(t+1, r-1) & \text { if } D_{t, r-1}-s_{t-1}^{u, v} \geq V \\ +\infty & \text { otherwise }\end{cases}
$$

We can compute all $\mathscr{L}[t, r]^{u, v(4)}$ in $O\left(w^{4}\right)$ time.

Sub-case 1.3: $r<\bar{t}_{u, v}$

The small ordering period is placed after period $r$, thus the ordering periods before $r$ are all large in which the ordered batches are all full. That is why we can easily compute the values of the entering stock levels $s_{t-1}^{u, v}$ and $s_{r-1}^{u, v}$ using Equation (2.14).

Let $\mathscr{L}[t, r]^{u, v(5)}$ be the minimum total cost to satisfy the demands $d_{t}, d_{t+1}, \ldots, d_{r-1}$ knowing that the periods $t$ and $r$ are large, and consecutive ordering periods and $r<\bar{t}_{u, v}$. Then, $\mathscr{L}[t, r]^{u, v(5)}$ is obtained in the same way as $\mathscr{L}[t, r]^{u, v(1)}$ using Equation (2.13) and switching the values of $s_{t-1}^{u, v}$ and $s_{r-1}^{u, v}$.

Case 2: $\bar{t}_{u, v}$ is small and $u=\bar{t}_{u, v}$

The subplan $(u, v)$ is composed, at the beginning of one replenishment cycle $[u, t]^{u, v(6)}$, of two 
consecutive ordering periods $u$ and $t$ such that $u$ is small and $t$ is large. The quantity $\bar{x}_{u, v}$ ordered in period $u$ is used to satisfy the demands $d_{u}, d_{u+1}, \ldots, d_{t-1}$, if we have $\bar{x}_{u, v} \geq D_{u, t-1}$.

Hence, we must firstly compute the minimum cost $\mathscr{L}[u, t]^{u, v(6)}$ of replenishment cycle $[u, t]^{u, v(6)}$ for $u<t \leq v$ :

$$
\mathscr{L}[u, t]^{u, v(6)}= \begin{cases}f_{u}+p_{u} \bar{x}_{u, v}+a_{u}+\sum_{k=u}^{t-1} h_{k}\left(\bar{x}_{u, v}-D_{u, k}\right) & \text { if } \bar{x}_{u, v} \geq D_{u, t-1} \\ +\infty & \text { otherwise }\end{cases}
$$

Secondly, we have the cost $\mathscr{L}[t, r]^{u, v(7)}$ of replenishment cycle $[t, r]^{u, v(7)}$ situated just after $[u, t]^{u, v(6)}$ such that $t$ and $r$ are large, and also are consecutive ordering periods, and the entering stock level in period $t$ is as follows:

$$
s_{t-1}^{u, v}= \begin{cases}\bar{x}_{u, v}-D_{u, t-1} & \text { if } \bar{x}_{u, v}-D_{u, t-1} \geq 0 \\ +\infty & \text { otherwise }\end{cases}
$$

Besides, the entering stock level in period $r$ is determined by Equation (2.12). The equation of $\mathscr{L}[t, r]^{u, v(7)}$ is the same as Equation (2.13) by replacing the value of $s_{t-1}^{u, v}$ with $u+1 \leq t<r \leq v$. Thirdly, we have several costs of type $\mathscr{L}[k, i]^{u, v(1)}$ of replenishment cycles $[k, i]^{u, v(1)}$ that are computed directly by Equation (2.13) with $r \leq k<i \leq v$.

\section{Case 3: $\bar{t}_{u, v}$ is large}

If $\bar{t}_{u, v}=u$, then the subplan $(u, v)$ is composed of large ordering periods and the fractional quantity is ordered at the beginning. For this reason, we just use the optimal costs $\mathscr{L}[t, r]^{u, v(1)}$ determined by Equation (2.13) with $t=u, \ldots, v-1$ and $r=t+1, \ldots, v$.

If $\bar{t}_{u, v}$ is the last ordering period in the subplan $(u, v)$, then the fractional quantity is ordered at the end of this subplan. Thus, we only need the optimal costs $\mathscr{L}[t, r]^{u, v(5)}$ (see Sub-case 1.3) with $t=u, \ldots, v-1$ and $r=t+1, \ldots, v$.

If $u<\bar{t}_{u, v}<b$, with $b$ being the last ordering period in $(u, v)$, then the latter is composed of three types of replenishment cycles. Firstly, we find several replenishment cycles of type $[k, i]^{u, v(5)}$ explained in Sub-case 1.3 in which the fractional quantity is ordered after all the possible values of $i$. We use the $\operatorname{costs} \mathscr{L}[k, i]^{u, v(5)}$ with $u \leq k<i \leq t \leq v-2$. Secondly, we have one replenishment cycle of type $[t, r]^{u, v(8)}$ such that $t$ is a large fractional ordering period and $r$ is a large full batch ordering period with $t<r \leq v-1$. We need to compute the cost $\mathscr{L}[t, r]^{u, v(8)}$ expressed as Equation (2.13) such that the value of $s_{t-1}^{u, v}$ is stated in Equation (2.14) and the one of $s_{r-1}^{u, v}$ is computed in Equation (2.12). Thirdly, there are many replenishment cycles of type $[l, j]^{u, v(1)}$ cited in Sub-case 1.1 knowing that $\bar{x}_{u, v}$ is ordered before $r$, where $r \leq l$. We use the costs $\mathscr{L}[l, j]^{u, v(1)}$ with $r \leq l \leq b<j \leq v$.

\section{How to compute $\mathscr{C}(u, v)$}

We show the computation of $\mathscr{C}(u, v)$ with $1 \leq u<v \leq w+1$. After the calculation of all the possible costs $\mathscr{L}[t, r]^{u, v(i)}$ in the subplan $(u, v)$ with $i=1, \ldots, 8$, we can now compute 6 possible values of the cost of subplan $(u, v)$ according to different situations (see Table 2.3 and Figure 2.4).

We have 6 situations of the subplan $(u, v)$ : 
Table 2.3: Costs $\mathscr{C}(u, v)$ in different situations

\begin{tabular}{|c|c|c|c|}
\hline \multicolumn{3}{|c|}{ Different situations of $(u, v)$} & $\begin{array}{l}\text { Possible } \\
\text { costs of } \\
(u, v)\end{array}$ \\
\hline \multirow{3}{*}{$\bar{t}_{u, v}$ is small } & \multirow{2}{*}{$\begin{array}{l}u \leq t<\bar{t}_{u, v}<r, t \text { and } r \text { are } 2 \\
\text { large consecutive ordering } \\
\text { periods }\end{array}$} & $\begin{array}{l}\bar{t}_{u, v} \text { avoids a shortage between } t \text { and } r \\
\text { with } r<v\end{array}$ & $\mathscr{C}_{1}(u, v)$ \\
\hline & & $\begin{array}{l}t_{u, v} \text { ensures a part of demand for peri- } \\
\text { ods } r \text { and thereafter with } r<v\end{array}$ & $\mathscr{C}_{2}(u, v)$ \\
\hline & \multicolumn{2}{|l|}{$\bar{t}_{u, v}=u$} & $\mathscr{C}_{3}(u, v)$ \\
\hline \multirow{3}{*}{$\bar{t}_{u, v}$ is large } & \multicolumn{2}{|l|}{$\bar{t}_{u, v}=u$} & $\mathscr{C}_{4}(u, v)$ \\
\hline & \multicolumn{2}{|c|}{$\bar{t}_{u, v}$ is the last ordering period in $(u, v)$} & $\mathscr{C}_{5}(u, v)$ \\
\hline & \multicolumn{2}{|c|}{$u<\bar{t}_{u, v}<$ last ordering period in $(u, v)$} & $\mathscr{C}_{6}(u, v)$ \\
\hline
\end{tabular}

Situation 1: We begin to define $\mathscr{C}_{1}(u, v)$ as the optimal total cost to satisfy the demand in periods $u, u+1, \ldots, v-1$, given that there is a small ordering period $\bar{t}_{u, v}$ between two large consecutive ordering periods $t$ and $r$, and the quantity ordered in $\bar{t}_{u, v}$ is consumed in the replenishment cycle of type $[t, r]^{u, v(3)}$ with $u \leq t<r \leq v$. The subplan $(u, v)$ in this situation is composed of different replenishment cycles. At the beginning, there are either several replenishment cycles of type $[t, r]^{u, v(5)}$ or nothing. Afterwards, there is one replenishment cycle of type $[t, r]^{u, v(3)}$. And next, there are either many replenishment cycles of type $[t, r]^{u, v(1)}$ or nothing.

To determine the total cost, we define $B_{j}^{u, v}$ as the optimal cost to satisfy the demands $d_{u}$, $d_{u+1}, \ldots, d_{j}$, such that $u, j+1$ and the ordering periods between them are large. In addition, the fractional period $\bar{t}_{u, v}$ is situated after $j+1$. The following dynamic programming algorithm determines the cost $B_{j}^{u, v}$ :

Recurrence relation. For $j=u, u+1, \ldots, v-1$,

$$
B_{j}^{u, v}=\min _{u-1 \leq i<j}\left\{B_{i}^{u, v}+\mathscr{L}[i+1, j+1]^{u, v(5)}\right\}
$$

Boundary condition. $B_{u-1}^{u, v}=0$

Objective. $B_{j}^{u, v}$

We also indicate $A_{t, k}^{u, v}$ as the optimal cost to satisfy the demands $d_{t}, d_{t+1}, \ldots, d_{k-1}$, such that $t$ and $k$ are large ordering periods, $\bar{t}_{u, v}$ (small) $<t$, the ordering period just before $t$ is either large or small with $s_{t-1}^{u, v}<V$, and the ones after $t$ are large. The objective is to compute the $\operatorname{cost} A_{t, v}^{u, v}$ which is obtained by the following dynamic program:

Recurrence relation. For $t=u, \ldots, v-1$ and for $k=t+1, \ldots, v$,

$$
A_{t, k}^{u, v}=\min _{t \leq i<k}\left\{A_{t, i}^{u, v}+\mathscr{L}[i, k]^{u, v(1)}\right\}
$$

Boundary condition. $A_{t, t}^{u, v}=0$

Objective. $A_{t, v}^{u, v}$

Hence,

$$
\mathscr{C}_{1}(u, v)=\min _{u-1 \leq i<v-2}\left\{B_{i}^{u, v}+\min \left\{\mathscr{L}[i+1, v]^{u, v(3)} ; \min _{i+3 \leq l \leq v-1}\left\{\mathscr{L}[i+1, l]^{u, v(3)}+A_{l, v}^{u, v}\right\}\right\}\right\}
$$


Situation 2: Define $\mathscr{C}_{2}(u, v)$ as the optimal total cost to satisfy the cumulative demand $D_{u, v-1}$, knowing that $\bar{t}_{u, v}$ is between two large consecutive ordering periods $t$ and $r$, and $\bar{x}_{u, v}$ is ordered to meet a part of demand situated after the replenishment cycle of type $[t, r]^{u, v(4)}$ $\left(V \leq s_{r-1}^{u, v}<2 V\right)$ with $u \leq t<r<v$.

The subplan $(u, v)$ in Situation 2 is composed of many replenishment cycles. At first, there are either replenishment cycles of type $[t, r]^{u, v(5)}$ or nothing. After that, there is one replenishment cycle of type $[t, r]^{u, v(4)}$. Then, there is one replenishment cycle of type $[t, r]^{u, v(2)}$, and eventually, there are either replenishment cycles of type $[t, r]^{u, v(1)}$ or nothing. Thus,

$$
\mathscr{C}_{2}(u, v)=\min _{u-1 \leq i<v-2}\left\{B_{i}^{u, v}+\min _{i+3 \leq l \leq v-1}\left\{\mathscr{L}[i+1, l]^{u, v(4)}+\min _{l+1 \leq t \leq v}\left\{\mathscr{L}[l, t]^{u, v(2)}+A_{t, v}^{u, v}\right\}\right\}\right\}
$$

Situation 3: For a given subplan $(u, v)$ such that $\bar{t}_{u, v}=u$ is small, we define $\mathscr{C}_{3}(u, v)$ as the optimal total cost of subplan $(u, v)$. One replenishment cycle of type $[u, t]^{u, v(6)}$ is the beginning of $(u, v)$ with $t \leq v$. After that we can find one replenishment cycle of type $[t, r]^{u, v(7)}$. Finally, there are either replenishment cycles of type $[t, r]^{u, v(1)}$ or nothing. The total cost of Situation 3 is presented as follows:

$$
\mathscr{C}_{3}(u, v)=\min \left\{\mathscr{L}[u, v]^{u, v(6)} ; \min _{u+1 \leq k \leq v-1}\left\{\mathscr{L}[u, k]^{u, v(6)}+\min _{k+1 \leq t \leq v}\left\{\mathscr{L}[k, t]^{u, v(7)}+A_{t, v}^{u, v}\right\}\right\}\right\}
$$

Situation 4: Define $\mathscr{C}_{4}(u, v)$ as the total optimal cost of $(u, v)$ given that $\bar{t}_{u, v}=u$ is large. In this subplan, we find only the replenishment cycles of type $[t, r]^{u, v(1)}$. Therefore,

$$
\mathscr{C}_{4}(u, v)=A_{u, v}^{u, v}
$$

Situation 5: In a subplan $(u, v)$ with the last and large ordering period $\bar{t}_{u, v}$, there are just replenishment cycles of type $[t, r]^{u, v(5)}$. Hence, the cost $\mathscr{C}_{5}(u, v)$ is obtained as follows:

$$
\mathscr{C}_{5}(u, v)=B_{v-1}^{u, v}
$$

Situation 6: Define $\mathscr{C}_{6}(u, v)$ as the optimal total cost of $(u, v)$ with a large fractional ordering period, $u<\bar{t}_{u, v}$ and $\bar{t}_{u, v}$ is prior to the last ordering period. There are replenishment cycles of type $[t, r]^{u, v(5)}$, one replenishment cycle of type $[t, r]^{u, v(8)}$ and replenishment cycles of type $[t, r]^{u, v(1)}$. Then,

$$
\mathscr{C}_{6}(u, v)=\min _{u \leq i<v-2}\left\{B_{i}^{u, v}+\min _{i+2 \leq l \leq v-1}\left\{\mathscr{L}[i+1, l]^{u, v(8)}+A_{l, v}^{u, v}\right\}\right\}
$$

After the calculation of all possible values of $\mathscr{C}(u, v)$ which can be computed in $O\left(w^{4}\right)$, the latter is obtained as follows:

$$
\mathscr{C}(u, v)=\min _{1 \leq i \leq 6}\left\{\mathscr{C}_{i}(u, v)\right\}
$$

The aim is to compute the optimal cost $C_{w}$ using the SP algorithm as mentioned in Equation (2.11). Thus, it solves the subproblem $\llbracket 1, w \rrbracket$ in $O\left(w^{4}\right)$ time and the LSP-B ${ }_{F T L} \mathrm{R}_{1}$ with $w>1$ in $O\left(T w^{3}\right)$.

\section{Numerical example for LSP-B $\mathbf{B}_{F T L} \mathbf{R}_{1}$ with $w>1$}

We present an illustrative example of the algorithm of $\mathrm{LSP}_{-} \mathrm{B}_{F T L} \mathrm{R}_{1}$ with $w>1$. We consider a time horizon of 4 periods in which the demands have to be satisfied in full and/or fractional batches of size $V=12$. Table 2.4 contains the data on the demand, cost and revenue. In the 
Table 2.4: Demand, different costs and revenue data

\begin{tabular}{lllllll}
\hline $\begin{array}{l}\text { Period } \\
t\end{array}$ & $d_{t}$ & $f_{t}$ & $p_{t}$ & $a_{t}$ & $h_{t}$ & $p_{t}^{b}$ \\
\hline 1 & 75 & 100 & 13 & 23 & 1 & - \\
2 & 9 & 250 & 10 & 23 & 1 & - \\
3 & 50 & 0 & 21 & 4 & 1 & - \\
4 & 23 & 0 & 13 & 3 & 1 & 9 \\
\hline
\end{tabular}

buyback contract considered in this example, we suppose that the unused items can be returned to the supplier only at the end of period 4.

Although return is allowed for the unused products at the end of period 4, using Property 2.5, we know that there is no return throughout the horizon under FTL cost structure. Table 3.1 shows the optimal plan of our example indicating that there is one independent subproblem $\llbracket 1,4 \rrbracket$ to optimize. It is interesting to note that there are two subplans $(1,4)$ and $(4,5)$. In $(1,4)$, there are a large ordering period (full) placed in period 1 and a small ordering period (fractional) situated in period 3. Therefore, this subplan belongs to the Case 1 (especially the Sub-case 1.2) by considering that the period 4 is a dummy large ordering period. We have $s_{3}^{1,4}=0<V$, thus the optimal cost of the subplan $(1,4)$ is $\mathscr{L}[1,4]^{1,4(3)}$. Concerning the subplan $(4,5)$, there is one ordering period placed in period 4 which is large and fractional. Hence, we take into account the Case 3 by considering that the periods 4 and 5 are large and consecutive ordering periods. The optimal cost of the subplan $(4,5)$ is $\mathscr{L}[4,5]^{4,5(1)}$.

Table 2.5: The optimal quantities of $\mathrm{LSP}_{-} \mathrm{B}_{F T L} \mathrm{R}_{1}$ with $w>1$

\begin{tabular}{lllll}
\hline $\begin{array}{l}\text { Period } \\
t\end{array}$ & $x_{t}$ & $s_{t}$ & $q_{t}$ & $A_{t}$ \\
\hline 1 & 132 & 57 & 0 & 11 \\
2 & 0 & 48 & 0 & 0 \\
3 & 2 & 0 & 0 & 1 \\
4 & 23 & 0 & 0 & 2 \\
\hline
\end{tabular}

\section{$2.4 \quad$ LSP-BR $_{1}$ with lost sales}

In LSP-BR 1 with $w \geq 1$ and lost sales, demands can be partially or totally lost. More accurately, lost sales involve allowing some orders not to be delivered if the total cost of ordering this demand is prohibitively large. We also study the four sub-cases listed in Section 2.3 under the concept of lost sales: $S P-B R_{1}$ with lost sales and only full batches; LSP-BR 1 with lost sales, only full batches and $w>1 ; S P-B R_{1}$ with lost sales and FTL cost structure; and finally LSP-BR 1 with lost sales, FTL cost structure and $w>1$. For these four sub-cases, we consider the same assumptions mentioned in Section 2.3, and we add the lost sales assumption wlog: $l_{t} V_{t} \geq p_{t} V_{t}+a_{t}$, $\forall \mathrm{t}=1, \ldots, T$. For the return policy $w>1$, we consider non-increasing lost sales costs over time. We recall that for $w=1$, we solve $T$ independent problems, each problem corresponding to one period. For $w>1$, there are $N$ independent problems to address. Each problem is expressed as $\llbracket(i-1) w+1, i w \rrbracket, \forall i=1, \ldots, N$. 


\subsubsection{SP-BR $\mathbf{R}_{1}$ with lost sales and only full batches (SP-B $\left.{ }_{L S, O F B} \mathbf{R}_{1}\right)$}

The $S P-B_{L S, O F B} R_{1}$ is a special case of $P_{B R_{1}}$. We fix $w$ to 1 and we modify Constraints $(2.3)$ by $q_{t} \geq 0, \forall t=1, \ldots, T$, Constraints (2.4) by $s_{t}=0, \forall t=1, \ldots, T$ and Constraints (2.8) by $x_{t}=A_{t} V_{t}, \forall t=1, \ldots, T$. We demonstrate two important dominance properties for this problem.

Property 2.9. There is an optimal solution such that a non-negative quantity is returned to the supplier in period $t \in\{1 . . T\}$ if and only if the lost sales amount drops to zero in that period. In our model, it is prohibited to have both $\left(L_{t}^{*}=0\right.$ and $q_{t}^{*}=0$ if $\left.d_{t} \bmod V_{t} \neq 0\right)$ or both $\left(L_{t}^{*}>0\right.$ and $\left.q_{t}^{*}>0\right)$.

Proof. Consider an optimal plan $\pi^{\prime}$ with either $\left(L_{t}^{\prime}>0\right.$ and $\left.q_{t}^{\prime}>0\right)$ or $\left(L_{t}^{\prime}=0\right.$ and $\left.q_{t}^{\prime}=0\right)$ in a period $t$.

Case 1. If $\left(L_{t}^{\prime}>0\right.$ and $\left.q_{t}^{\prime}>0\right)$, then, one can reduce $L_{t}^{\prime}$ to $L_{t}^{\prime}-\min \left\{L_{t}^{\prime}, q_{t}^{\prime}\right\}$ and $q_{t}^{\prime}$ to $q_{t}^{\prime}-\min \left\{L_{t}^{\prime}, q_{t}^{\prime}\right\}$, by retaining the solution's feasibility. This marks a decrease of the total cost $\mathscr{C}^{\prime}$ of the plan $\pi^{\prime}$ by $\left(l_{t}-p_{t}^{b}\right) \min \left\{L_{t}^{\prime}, q_{t}^{\prime}\right\}$. By the assumptions of buyback contract and lost sales, we have $l_{t}>p_{t}^{b}$. Therefore, the solution with $L_{t}^{\prime}>0$ and $q_{t}^{\prime}>0$ cannot be optimal - a contradiction.

Case 2. If $L_{t}^{\prime}=0$ and $q_{t}^{\prime}=0$, then, $x_{t}^{\prime}=d_{t}$ according to Constraints (2.2). However, we have a batch ordering problem, and hence this case is impossible when $d_{t} \bmod V_{t} \neq 0$.

Property 2.10. The optimal policy $\pi^{*}$ orders a quantity $x_{t}^{*}$ which can take, in every period $t$, one of the three following values: $x_{t}^{*(1)}=0$, or $x_{t}^{*(2)}=\left\lfloor\frac{d_{t}}{V_{t}}\right\rfloor V_{t}$, or $x_{t}^{*(3)}=\left\lceil\frac{d_{t}}{V_{t}}\right\rceil V_{t}$.

Proof. Suppose that there is an optimal policy $\pi^{\prime}$ with the optimal ordering quantity $x_{t}^{\prime}$ such that $0<x_{t}^{\prime}<\left\lfloor\frac{d_{t}}{V_{t}}\right\rfloor V_{t}$, or $\left\lfloor\frac{d_{t}}{V_{t}}\right\rfloor V_{t}<x_{t}^{\prime}<\left\lceil\frac{d_{t}}{V_{t}}\right\rceil V_{t}$ or $x_{t}^{\prime}>\left\lceil\frac{d_{t}}{V_{t}}\right\rceil V_{t}$ in a period $t$. In each interval, we demonstrate the non optimality compared to $x_{t}^{*(1)}, x_{t}^{*(2)}$ and $x_{t}^{*(3)}$.

Case 1. If $0<x_{t}^{\prime}<\left\lfloor\frac{d_{t}}{V_{t}}\right\rfloor V_{t}$, then $x_{t}^{\prime}=\left(\left\lfloor\frac{d_{t}}{V_{t}}\right\rfloor-\lambda\right) V_{t}, A_{t}^{\prime}=\left\lfloor\frac{d_{t}}{V_{t}}\right\rfloor-\lambda, q_{t}^{\prime}=0$ by using Property 2.9 and Constraints (2.2), and $L_{t}^{\prime}=d_{t}-\left\lfloor\frac{d_{t}}{V_{t}}\right\rfloor V_{t}+\lambda V_{t}$ with $\lambda \in \mathbb{N}^{*}$ and $0<\lambda<\left\lfloor\frac{d_{t}}{V_{t}}\right\rfloor$. Hence, the total cost $\mathscr{C}^{\prime}$ in which $\left.x_{t}^{\prime} \in\right] 0 ;\left\lfloor\frac{d_{t}}{V_{t}}\right\rfloor V_{t}\left[\right.$ is ordered in period $t$ depends on the total cost $\mathscr{C}^{*(1)}$ where 0 is ordered in period $t$, and is computed as follows:

$$
\mathscr{C}^{\prime}=\mathscr{C}^{*(1)}+f_{t}-\left(\left\lfloor\frac{d_{t}}{V_{t}}\right\rfloor-\lambda\right)\left(V_{t}\left(l_{t}-p_{t}\right)-a_{t}\right)
$$

If $f_{t}>\left(\left\lfloor\frac{d_{t}}{V_{t}}\right\rfloor-\lambda\right)\left(V_{t}\left(l_{t}-p_{t}\right)-a_{t}\right)$, then the solution $x_{t}^{\prime}$ cannot be optimal - a contradiction.

If $f_{t}=\left(\left\lfloor\frac{d_{t}}{V_{t}}\right\rfloor-\lambda\right)\left(V_{t}\left(l_{t}-p_{t}\right)-a_{t}\right)$ for a value of $\lambda$ such as $1 \leq \lambda \leq\left\lfloor\frac{d_{t}}{V_{t}}\right\rfloor-1$, then we still have an alternative optimal solution that can satisfy the property.

If $f_{t}<\left(\left\lfloor\frac{d_{t}}{V_{t}}\right\rfloor-\lambda\right)\left(V_{t}\left(l_{t}-p_{t}\right)-a_{t}\right)$, then we proceed to compare the cost $\mathscr{C}^{\prime}$ versus the total cost $\mathscr{C}^{*(2)}$ where $\left\lfloor\frac{d_{t}}{V_{t}}\right\rfloor V_{t}$ is ordered in period $t$ as follows:

$$
\mathscr{C}^{\prime}=\mathscr{C}^{*(2)}+\lambda\left(V_{t}\left(l_{t}-p_{t}\right)-a_{t}\right)
$$

With the assumption on the lost sales cost $l_{t} \geq p_{t}+\frac{a_{t}}{V_{t}}$, the current solution $0<x_{t}^{\prime}<\left\lfloor\frac{d t}{V_{t}}\right\rfloor V_{t}$ 
cannot be optimal when $l_{t}>p_{t}+\frac{a_{t}}{V_{t}}$. For $l_{t}=p_{t}+\frac{a_{t}}{V_{t}}$, there is a new optimal solution $\left(x_{t}^{*(2)}=\right.$ $\left.\left\lfloor\frac{d_{t}}{V_{t}}\right\rfloor V_{t}\right)$ that satisfies the property.

Case 2. If $\left\lfloor\frac{d_{t}}{V_{t}}\right\rfloor V_{t}<x_{t}^{\prime}<\left\lceil\frac{d_{t}}{V_{t}}\right\rceil V_{t}$, then $x_{t}^{\prime}$ cannot be expressed as a multiple of the batch size $V_{t}$ which gives an infeasible solution - contradiction.

Case 3. If $x_{t}^{\prime}>\left\lceil\frac{d_{t}}{V_{t}}\right\rceil V_{t}$, then $L_{t}^{\prime}=0$. However, we showed that in $S P-B_{O F B} R_{1}$ without lost sales, the optimal procurement quantity in a period $t$ can only be $\left\lceil\frac{d_{t}}{V_{t}}\right\rceil V_{t}$. Hence, we cannot obtain the optimality with $x_{t}^{\prime}>\left\lceil\frac{d_{t}}{V_{t}}\right\rceil V_{t}$.

Properties 2.9 and 2.10 allow us to develop an efficient algorithm in order to find the optimal plan for the $S P-B_{L S, O F B} R_{1}$. The key idea is to divide the time horizon into $T$ independent stages. Each stage corresponds to a period $t$, in which, by Property 2.10, the retailer procures either 0 or $\left\lfloor\frac{d_{t}}{V_{t}}\right\rfloor V_{t}$ or $\left\lceil\frac{d_{t}}{V_{t}}\right\rceil V_{t}$. Then, by Property 2.9 and following the constraints of our problem, we can compute $y_{t}^{*}, A_{t}^{*}, L_{t}^{*}$ and $q_{t}^{*}$ related to each possible optimal ordered quantity $x_{t}^{*}$ in every period $t$. For example, if $x_{t}^{*}=0$, then by using Constraints (2.2), we obtain $d_{t}-L_{t}^{*}+q_{t}^{*}=0$. Using Property 2.9, if $L_{t}^{*}=0$ then $q_{t}^{*}=-d_{t}$ which contradicts the feasibility constraint of $q_{t}$, and if $q_{t}^{*}=0$ then $L_{t}^{*}=d_{t}$ which is trivial. After that, we calculate the total cost of each possible optimal ordered quantity $x_{t}^{*}$ in every period $t$. Hence, in each period $t$, we have three total costs $C_{t}^{(1)}, C_{t}^{(2)}$ and $C_{t}^{(3)}$ which correspond to the three possible optimal procurement quantities $x_{t}^{*(1)}$, $x_{t}^{*(2)}$ and $x_{t}^{*(3)}$ respectively.

$$
\begin{aligned}
& C_{t}^{(1)}:=l_{t} d_{t} \\
& C_{t}^{(2)}:=f_{t}+p_{t}\left\lfloor\frac{d_{t}}{V_{t}}\right\rfloor V_{t}+a_{t}\left\lfloor\frac{d_{t}}{V_{t}}\right\rfloor+l_{t}\left(d_{t}-\left\lfloor\frac{d_{t}}{V_{t}}\right\rfloor V_{t}\right) \\
& C_{t}^{(3)}:=f_{t}+p_{t}\left\lceil\frac{d_{t}}{V_{t}}\right\rceil V_{t}+a_{t}\left\lceil\frac{d_{t}}{V_{t}}\right\rceil-p_{t}^{b}\left(\left\lceil\frac{d_{t}}{V_{t}}\right\rceil V_{t}-d_{t}\right)
\end{aligned}
$$

Besides, we must choose the minimum cost. We define $C^{\text {min }}(t)$ as the minimum cost among $C_{t}^{(1)}, C_{t}^{(2)}$ and $C_{t}^{(3)}$ in every period $t$.

$$
\begin{aligned}
& C^{\min }(t):=\min \left\{C_{t}^{(1)}, C_{t}^{(2)}, C_{t}^{(3)}\right\} \\
& \mathscr{C}^{*}=\sum_{t=1}^{T} C^{\min }(t)
\end{aligned}
$$

We present $\mathscr{C}^{*}$ as the sum of $C^{\text {min }}(t)$ from period 1 to period $T$. Consequently, $\mathscr{C}^{*}$ represents the optimal objective value of the $S P-B_{L S, O F B} R_{1} . \mathscr{C}^{*}$ is computed by an $O(T)$ time algorithm.

\subsubsection{LSP-BR $_{1}$ with lost sales, only full batches and $w>1\left(\mathbf{L S P}-\mathbf{B}_{L S, O F B} \mathbf{R}_{1}\right.$ with $w>1$ )}

To obtain LSP-B ${ }_{L S, O F B} \mathrm{R}_{1}$ with $w>1$, we replace in $P_{B R_{1}}$ Constraints (2.8) with $x_{t}=A_{t} V$, where $V_{t}=V, \forall t=1, \ldots, T$. In this problem, we take into account the aspect of lost sales and we assume non-increasing lost sales costs over time. We note that the single-item LSP with immediate lost sales is studied by Aksen et al. (2003), which constitutes a special case of our problem with $w=T$ and $V_{t}=1$. They provide a dynamic programming algorithm with a complexity in $\mathrm{O}\left(T^{2}\right)$ time.

In our algorithm for LSP-B $\mathrm{B}_{L S, O F B} \mathrm{R}_{1}$ with $w>1$, we use the same decomposition scheme into subplans $(u, v)$ as previously. Therefore, we must determine the minimum costs $\mathscr{C}(u, v)$ of all subplans $(u, v)$ to decide how much of demand to satisfy and to lose for periods $u, u+1, \ldots, v-1$, with $1 \leq u<v \leq w+1$. Then, we can obtain the total optimal cost $C_{w}$ of the subproblem $\llbracket 1, w \rrbracket$, in which each demand can be either totally satisfied, or partially lost, or totally lost with $w+1$ 
being a regeneration period, by using the same $\mathrm{SP}$ algorithm proposed for $\mathrm{LSP}-\mathrm{B}_{F T L} \mathrm{R}_{1}$ with $w>1$ in Section 2.3.4. In the following, we will prove that all the $\mathscr{C}(u, v)$ values can be obtained recursively in $O\left(w^{4}\right)$ time for the subproblem $\llbracket 1, w \rrbracket$. Therefore, the overall complexity of the LSP-B ${ }_{L S, O F B} \mathrm{R}_{1}$ with $w>1$ algorithm is $O\left(T w^{3}\right)$ time. At the end, we present an illustrative example for this algorithm.

The key idea behind computing the cost $\mathscr{C}(u, v)$ is to decompose the subplan $(u, v)$ into different cycles. At the beginning, we can find the replenishment cycles of type $[t, r]^{u, v(5)}$ such that $t$ and $r$ are two consecutive ordering periods without lost sales for $u \leq t<r \leq v$ (see Sub-case 1.3 of Section 2.3.4). After that, if $v<w+1$, then there is what we call a loss and replenishment cycle denoted by $\langle k, v\rangle^{u, v}$ such that $k$ is the last ordering period in $(u, v)$ and $v-1$ is the loss period in which the amount of unmet demand is strictly greater than 0 , for $u \leq k<v<w+1$. If $v=w+1$, then there is either one loss and replenishment cycle $\langle k, w+1\rangle^{u, w+1}$ for $u \leq k<w+1$, or one return and replenishment cycle denoted by $\|k, w+1\|^{u, w+1}$ such that $k$ is the last ordering period in $(u, w+1)$ and $w$ is the return period for $u \leq k<w+1$.

\section{Properties and corollaries}

We will first establish some optimality properties for our subproblem $\llbracket 1, w \rrbracket$ in order to propose an $O\left(w^{4}\right)$ time algorithm.

We use Property 2.9 which affirms that $L_{w} q_{w}=0$. In other words, the retailer cannot return and lose at the same time. We give some other properties in the following:

Property 2.11. In any optimal solution, the starting inventory level $s_{r-1}$ of an ordering period $r$ is less than $V$ units $\left(s_{r-1}<V\right)$, for $r=1, \ldots, w+1$.

Proof. We suppose for the sake of contradiction that $s_{r-1} \geq V$. This latter implies that we have an ordering period $t$, which is just before $r$, with at least $V$ units in stock between the end of period $t$ and the beginning of period $r$. Since the retailer only orders in full batches, we have $x_{t} \geq V$ and $x_{r} \geq V$. The proof of this contradiction is the same given in the proof of Property 2.7 , case 1 . In the calculation of the optimal and non-optimal cost difference, we keep the same lost sales quantity in each period and we just change the ordered quantities in period $t$ and $r$ and the ending inventory levels between periods $t$ and $r-1$.

Property 2.12. In any optimal solution, for a null ordering period $i\left(x_{i}=0\right)$, if $s_{i-1} \geq V$ then $L_{i}<V$.

Proof. Let us consider an optimal policy $\pi^{\prime}$ with a null ordering period $i\left(x_{i}^{\prime}=0\right)$ having an entering stock level $s_{i-1}^{\prime} \geq V$ and a lost sales quantity of $L_{i}^{\prime} \geq V$. Let us consider $L_{i}^{\prime}=n V+\epsilon$ with $\epsilon \geq 0$ and $n \in \mathbb{N}^{*}$. Since $i$ is a null ordering period and $s_{i-1}^{\prime} \geq V$, then, on the one hand there is an ordering period $t$ before $i(t \leq i-1)$, and on the other hand $s_{k}^{\prime} \geq V$ such that $k$ represents any period between $t$ and $i-1$. We can consider two other policies:

- Policy $\pi^{(1)}$ orders $V$ additional units in period $t$, and loses one batch less in period $i$, that is, $x_{t}^{(1)}=x_{t}^{\prime}+V$ and $L_{i}^{(1)}=L_{i}^{\prime}-V$. This first new policy is feasible because $L_{i}^{\prime} \geq V$ in the optimal policy $\pi^{\prime}$. 
- Policy $\pi^{(2)}$ orders one fewer batch in period $t$, and loses $V$ additional units in period $i$, that is, $x_{t}^{(2)}=x_{t}^{\prime}-V$ and $L_{i}^{(2)}=L_{i}^{\prime}+V$. This policy remains feasible because $x_{t}^{(2)} \geq 0$ and $s_{k}^{(2)} \geq 0$ for any period $k$ between $t$ and $i-1$.

Now, we compute the cost of each policy based on the cost of the policy $\pi^{\prime}$. We have:

$$
\begin{aligned}
& \mathscr{C}\left(\pi^{(1)}\right)=\mathscr{C}\left(\pi^{\prime}\right)+V\left(p_{t}+\frac{a_{t}}{V}+\sum_{k=t}^{i-1} h_{k}-l_{i}\right) \\
& \mathscr{C}\left(\pi^{(2)}\right)=\mathscr{C}\left(\pi^{\prime}\right)-V\left(p_{t}+\frac{a_{t}}{V}+\sum_{k=t}^{i-1} h_{k}-l_{i}\right)
\end{aligned}
$$

Since $\pi^{\prime}$ is an optimal policy, we must have $l_{i}=p_{t}+\frac{a_{t}}{V}+\sum_{k=t}^{i-1} h_{k}$. Thereafter, we propose a policy $\pi^{(3)}$ which orders $n V$ additional units in period $t$, and loses $n$ batches in period $i$, that is, $x_{t}^{(3)}=x_{t}^{\prime}+n V$ and $L_{i}^{(3)}=L_{i}^{\prime}-n V$ and we obtain $\mathscr{C}\left(\pi^{(3)}\right)=\mathscr{C}\left(\pi^{\prime}\right)$. Therefore, the optimal policy $\pi^{\prime}$ can be modified in such a way that the lost sales quantity in period $i$ is less than $V$ units.

Property 2.13. There exists an optimal policy such that $L_{t} s_{t}=0$ for each $t=1,2, \ldots, w$. If we decide not to satisfy a positive amount of demand in period $t$, the stock level will drop to zero at the end of that period. This also means that if we have a positive stock at the end of a period, the latter cannot be a lost sales period.

This property implies that in each subplan $(u, v)$, there is at most one loss period which is $v-1$, because this is the only period with a null outgoing stock level. Any possible lost sales quantity in the subplan $(u, v)$ must be of the form: $L_{v-1}^{u, v}=D_{u, v-1}-\left\lfloor\frac{D_{u, v-1}}{V}\right\rfloor V+n V$ such that $n \in \mathbb{N}$ and $n \leq\left\lfloor\frac{D_{u, v-1}}{V}\right\rfloor$.

Proof. Let us consider an optimal policy $\pi^{\prime}$ with a subplan $(u, v)$ having at the same time $s_{i}^{\prime}>0$ and $L_{i}^{\prime}>0$, for $u \leq i<v-1$. Let $\tau=\min \left\{L_{i}^{\prime}, \min _{t=i, \ldots, v-2} s_{t}^{\prime}\right\}$.

If we decrease $L_{i}^{\prime}$ and $s_{t}^{\prime}$ for $t=i, \ldots, v-2$ by $\tau$ and increase $L_{v-1}^{\prime}$ by $\tau$, we obtain another policy $\pi^{*}$ in which the modified solution remains feasible because we always have $L_{v-1}^{\prime}+s_{v-2}^{\prime} \leq d_{v-1}$ obtained by the balancing constraints. Furthermore, the total cost of $\pi^{*}$ can be computed as follows:

$$
\mathscr{C}\left(\pi^{*}\right)=\mathscr{C}\left(\pi^{\prime}\right)+\tau\left(l_{v-1}-l_{i}-\sum_{t=i}^{v-2} h_{t}\right)
$$

Since, we have $l_{v-1} \leq \ldots \leq l_{i} \leq \ldots \leq l_{u}$ (due to the assumption of non-increasing lost sales costs), then it is easy to see that $\mathscr{C}\left(\pi^{*}\right) \leq \mathscr{C}\left(\pi^{\prime}\right)$. If $\tau=L_{i}^{\prime}$, then the policy $\pi^{*}$ can be optimal in which there is one loss period situated in $v-1$. Now, if $\tau=\min _{t=i, \ldots, v-2}\left\{s_{t}^{\prime}\right\}=s_{b}^{\prime}$, with $b \in\{i, \ldots, v-2\}$, then the subplan $(u, v)$ is divided into at least two subplans $(u, b+1)$ and $(b+1, v)$. The case where there are several periods $b$ is easy to discuss in the same way.

In the subplan $(u, b+1)$, there is one loss period situated in $i$ with $u \leq i \leq b$. In this case we have $L_{i}^{*} s_{i}^{*}>0$. Whereas, in the subplan $(b+1, v)$ there is one loss period placed in $v-1$. Now, we will prove that if we have a loss period in the subplan $(u, b+1)$, then it must be $b$ which has a null ending stock level. To get this, define $L_{i}^{*}=L_{i}^{\prime}-s_{b}^{\prime}$, for $k=i, \ldots, b, s_{k}^{*}=s_{k}^{\prime}-s_{b}^{\prime}$ and $L_{b}^{*}=0$ obtained by $\pi^{*}$. Let $\tau_{1}=\min \left\{L_{i}^{*}, \min _{k=i, \ldots, b-1} s_{k}^{*}\right\}$. 
If we decrease $L_{i}^{*}$ and $s_{k}^{*}$ by $\tau_{1}$ such that $k=i, \ldots, b-1$, and increase $L_{b}^{*}$ by $\tau_{1}$, we obtain the policy $\pi^{* *}$ by maintaining the feasibility of the solution because we always have $L_{b}^{*}+s_{b-1}^{*} \leq d_{b}$. Hence, the total cost of $\pi^{* *}$ is as follows:

$$
\mathscr{C}\left(\pi^{* *}\right)=\mathscr{C}\left(\pi^{\prime}\right)+s_{b}^{*}\left(l_{v-1}-l_{i}-\sum_{t=i}^{v-2} h_{t}\right)+\tau_{1}\left(l_{b}-l_{i}-\sum_{k=i}^{b-1} h_{k}\right)
$$

Under non-increasing lost sales costs, we have $\mathscr{C}\left(\pi^{* *}\right) \leq \mathscr{C}\left(\pi^{\prime}\right)$.

If $\tau_{1}=L_{i}^{*}$, then $b$ is the only loss period in the subplan $(u, b+1)$. And if $\tau_{1}=\min _{k=i, \ldots, b-1}\left\{s_{k}^{\prime}\right\}=$ $s_{j}^{\prime}$, then we repeat the same procedure above until having the optimality.

By definition of $\tau$, the above optimal solution can be modified in such a way that either the number of loss periods among periods $u, u+1, \ldots, v-1$ is reduced to 1 and the latter is placed in $v-1$, or $(u, v)$ is divided into several subplans because there are new regeneration periods among periods $i+1, \ldots, v-1$. The result follows.

We have the following corollary which will be used to compute the total optimal cost of the subplan $(u, v)$ :

Corollary 2.1. If $v-1$ is an ordering period and there is a positive lost sales quantity, then $L_{v-1}^{u, v}=D_{u, v-1}-\left\lfloor\frac{D_{u, v-1}}{V}\right\rfloor V$ due to the lost sales assumption $l_{v-1} V \geq p_{v-1} V+a_{v-1}$ (Section 2.2). In addition, if $v-1$ is a non ordering period and $L_{v-1}>0$, then $L_{v-1}^{u, v}=d_{v-1}-s_{v-2}$.

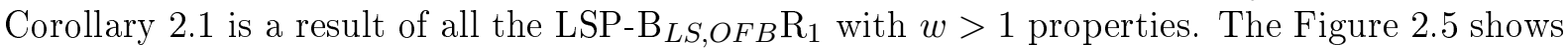

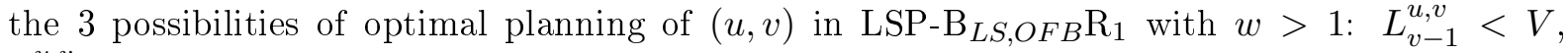
$L_{v-1}^{u, v} \geq V$, and if $v-1=w, q_{w}>0$.

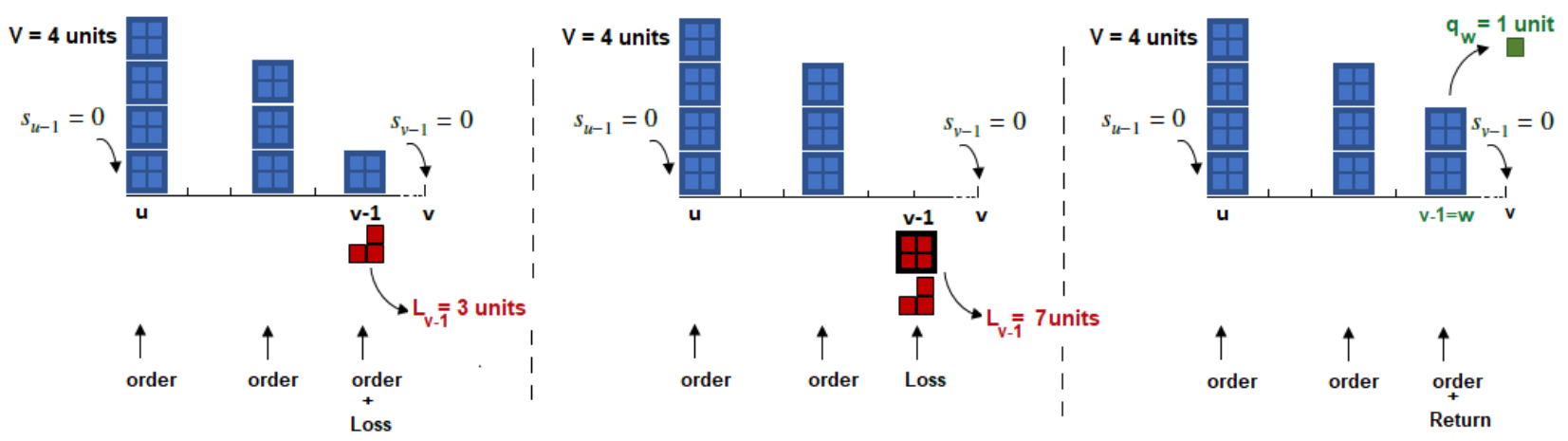

Figure 2.5: Possibilities of optimal planning of $(u, v)$ in $\operatorname{LSP}_{-\mathrm{B}_{L S, O F B}} \mathrm{R}_{1}$ with $w>1$

\section{How to compute $\mathscr{C}(u, v)$}

Recall that inside the $\operatorname{subplan}(u, v)$ such that $1 \leq u<v \leq w+1$, there is at most one loss period which is the period $v-1$ and for any ordering period $t, s_{t-1}^{u, v}=\left\lceil\frac{D_{u, t-1}}{V}\right\rceil V-D_{u, t-1}$, based on the fact that there is no lost sales between the periods $u$ and $v-2$, and on Property 2.11. Furthermore, in each subplan $(u, w+1)$, the demand in period $w$ will be partially or fully lost if and only if the return is not made in that period, and if $D_{u, w} \bmod V \neq 0$. Otherwise, we have $L_{w}^{u, w+1} \geq 0$ if and only if $q_{w}=0$. 
To find the optimal cost of the subplan $(u, v)$, we need to compute, first, the minimum total cost $G_{r}^{u, v}$ to satisfy the demands $d_{u}, d_{u+1}, \ldots, d_{r-1}$, for $1 \leq u<r \leq v \leq w+1$, such that $r$ must be the last ordering period and/or the loss or return period of $(u, v)$. Then, the period $r$ can be either the last ordering period if $r<v-1 \leq w$, or a loss period which can also be the last ordering period if $r=v-1<w$, or a loss or a return period in which the retailer can order some quantity if $r=v-1=w$, or the last ordering period if $r=v=w+1$.

To find the values of $G_{r}^{u, v}$, we need to compute the cost of each possible replenishment cycle of type $[t, r]^{u, v(5)}$ for the values of the entering stocks in periods $t$ and $r$. In LSP-B $\mathrm{B}_{L S, O F B} \mathrm{R}_{1}$ with $w>1$, all ordering periods are large with only full batches. Thus, the optimal total cost to satisfy the demands $d_{t}, d_{t+1}, \ldots, d_{r-1}$ with $t$ and $r$ being 2 consecutive ordering periods is $\mathscr{L}[t, r]^{u, v(5)}$, which is detailed in Section 2.3.4. Hence, the dynamic programming formulation of $G_{r}^{u, v}$ is given as follows:

Recurrence relation. For $r=u+1, \ldots, v$,

$$
G_{r}^{u, v}=\min _{u \leq t<r}\left\{G_{t}^{u, v}+\mathscr{L}[t, r]^{u, v(5)}\right\}
$$

Boundary condition. $G_{u}^{u, v}=0$

Objective. $G_{r}^{u, v}$

All $G_{r}^{u, v}$ values can be computed in time $O\left(w^{4}\right)$. Define the indicator functions $\mathbb{1}_{a}$ and $\mathbb{1}_{a_{i}}^{i}$, as follows:

$$
\mathbb{1}_{a}=\left\{\begin{array}{ll}
1 & \text { if } a \text { is true, } \\
+\infty & \text { otherwise }
\end{array} \quad \mathbb{1}_{a_{i}}^{i}= \begin{cases}1 & \text { if } a_{i} \text { is true } \\
+\infty & \text { otherwise }\end{cases}\right.
$$

In the case where $r$ is the last ordering period in the subplan $(u, v)$ and $v-1$ is the loss period with $L_{v-1}^{u, v}<V$, we have to compute, second, the total holding cost $H_{r}^{u, v}$ from the end of period $r$ until the beginning of $v-1$ to satisfy the demand of periods $r, r+1, \ldots, v-2$, for $u \leq r<v-1$. Hence, the quantity ordered in period $r$ in $(u, v)$ is equal to $\left\lfloor\frac{D_{r, v-1}-s_{r-1}^{u, v}}{V}\right\rfloor V$, and the quantity stored in each period $i$ between $r$ and $v-2$ is denoted by $a_{i}$ such that $a_{i}=$ $\left\lfloor\frac{D_{r, v-1}-s_{r-1}^{u, v}}{V}\right\rfloor V-\left(D_{r, i}-s_{r-1}^{u, v}\right)$. Then,

$$
\left\{\begin{array}{l}
H_{r}^{u, v}=H_{r+1}^{u, v}+\mathbb{1}_{a_{r}>0}^{r} h_{r} a_{r} \quad \text { if } u \leq r<v-1 \\
H_{v-1}^{u, v}=0
\end{array}\right.
$$

The time required to precompute all $H_{r}^{u, v}$ values is bounded by $O\left(w^{3}\right)$.

After that, we can compute the possible values of $\mathscr{C}(u, v)$ in $O\left(w^{4}\right)$ time. Thus, for $1 \leq u<$ $v \leq w+1$ :

$$
\begin{aligned}
& \mathscr{C}(u, v)=\min \left\{\operatorname { m i n } _ { u \leq r \leq v - 1 \leq w } \left\{G_{r}^{u, v}+\min \left\{\mathbb{1}_{D_{r, v-1}-s_{r-1}^{u, v} \geq L_{v-1}^{u, v}} f_{r}+\left(p_{r}+\frac{a_{r}}{V}\right)\left\lfloor\frac{D_{r, v-1}-s_{r-1}^{u, v}}{V}\right\rfloor V\right.\right.\right. \\
& +H_{r}^{u, v}+l_{v-1} L_{v-1}^{u, v} ; \\
& \left.\left.\mathbb{1}_{r=v-1} \mathbb{1}_{d_{v-1}-s_{v-2}^{u, v} \geq L_{v-1}^{u, v}} l_{v-1}\left|d_{v-1}-s_{v-2}^{u, v}\right|\right\}\right\} ; \\
& \left.\mathbb{1}_{v=w+1} G_{w+1}^{u, v}-p_{w}^{b}\left(\left\lceil\frac{D_{u, w}}{V}\right\rceil V-D_{u, w}\right)\right\}
\end{aligned}
$$

To see how the above relation is constructed, we consider in each subplan $(u, v)$ with $u<v$, that $u$ is the first ordering period and all the demands $d_{u}, d_{u+1}, \ldots, d_{r-1}$ must be satisfied with the minimum cost $G_{r}^{u, v}$ in which the period $r$ can be the last ordering period and/or the loss or 
return period, for $1 \leq u \leq r \leq v \leq w+1$. We have 3 situations of $(u, v)$. In the first situation, the subplan has a lost sales quantity less than the batch size $V$. Then, the retailer orders in period $r$ a quantity of $\left\lfloor\frac{D_{r, v-1}-s_{r-1}^{u, v}}{V}\right\rfloor V$ to satisfy the demands $d_{r}, d_{r+1}, \ldots, d_{v-2}$ and loses in $v-1$ a quantity of $L_{v-1}^{u, v}=D_{u, v-1}-\left\lfloor\frac{D_{u, v-1}}{V}\right\rfloor V$. In this case, the position of the period $r$ belongs to $\{u, \ldots, v-1\}$ with $v-1 \leq w$. In the second situation, the lost sales quantity in $v-1$ is greater than or equal to $V$. Then, $v-1$ is a null ordering period and there is a loss of $d_{v-1}-s_{v-2}^{u, v}$. In this case, we have $r=v-1 \leq w$. In the third situation, the subplan $(u, v)$ possesses a procurement surplus and a return of $\left[\frac{D_{u, w}}{V}\right] V-D_{u, w}$. Then, we have $r=v=w+1$.

After predetermining all the possible $\mathscr{C}(u, v)$ values, we can compute the optimal total cost $C_{w}$ to satisfy or to lose each demand in the subproblem $\llbracket 1, w \rrbracket$ with only full batch, expressed in Formula (2.11), in $O\left(w^{4}\right)$ time. Therefore, the running time of the dynamic programming algorithm proposed for LSP-B $\mathrm{B}_{L S, O F B} \mathrm{R}_{1}$ with $w>1$ is $O\left(T w^{3}\right)$.

\section{Numerical example for LSP-B LS,OFB $_{1} \mathbf{R}_{1}$ with $w>1$}

We use the same example presented for the algorithm of LSP-B ${ }_{F T L} \mathrm{R}_{1}$ with $w>1$ (see Section 2.3.4) by adding four lost sales costs, $l_{1}=23.5, l_{2}=22.3, l_{3}=21.8$ and $l_{4}=21.5$. In this problem, the demands can be partially or fully satisfied in full batches, or can be fully lost.

Table 2.6 shows the optimal solutions of this problem. We have the same decomposition of subplans $(1,4)$ and $(4,5)$. In $(1,4)$, the period 1 is the only ordering period which is large and full, and the period 3 is the only loss period in which the quantity of unmet demand is lower than the batch size 12. The optimal cost of the subplan $(1,4)$ is the cost of the loss and replenishment cycle $\langle 1,4\rangle^{1,4}$. In $(4,5)$, the period 4 is at the same time a large and full ordering period and a return period. The optimal cost of this subplan is the cost of the return and replenishment cycle $\|4,5\|^{4,5}$.

Table 2.6: The optimal quantities of LSP-B $\mathrm{B}_{L S, O F B} \mathrm{R}_{1}$ with $w>1$

\begin{tabular}{llllll}
\hline $\begin{array}{l}\text { Period } \\
t\end{array}$ & $x_{t}$ & $s_{t}$ & $L_{t}$ & $q_{t}$ & $A_{t}$ \\
\hline 1 & 132 & 57 & 0 & 0 & 11 \\
2 & 0 & 48 & 0 & 0 & 0 \\
3 & 0 & 0 & 2 & 0 & 0 \\
4 & 24 & 0 & 0 & 1 & 2 \\
\hline
\end{tabular}

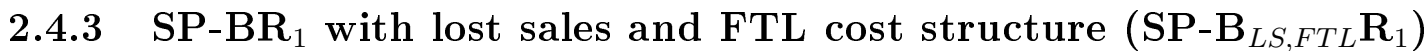

Compared to the case $S P-B_{L S, O F B} R_{1}$, we do not consider any specific assumption on the batches. The ordered quantities can now be in fractional batches. Two important dominance properties are given as follows:

Property 2.14. In any optimal solution, there is no returned quantity, i.e. $q_{t}^{*}=0, \forall t=1, \ldots, T$.

Proof. With the assumption of $p_{t}>p_{t}^{b}$, the solution with $q_{t}=0$ is optimal.

Property 2.15. The optimal plan $\pi^{*}$ orders either 0 or $\left\lfloor\frac{d_{t}}{V_{t}}\right\rfloor V_{t}$ or $d_{t}$ in every period $t$. We 
indicate the three possible optimal ordered quantities as follows: $x_{t}^{*(1)}=0, x_{t}^{*(2)}=\left\lfloor\frac{d_{t}}{V_{t}}\right\rfloor V_{t}$ and $x_{t}^{*(3)}=d_{t}$.

Proof. Suppose that there is an optimal plan $\pi^{\prime}$ ordering a quantity $x_{t}^{\prime}$ in a period $\mathrm{t}$ such that $0<x_{t}^{\prime}<\left\lfloor\frac{d_{t}}{V_{t}}\right\rfloor V_{t}$, or $\left\lfloor\frac{d_{t}}{V_{t}}\right\rfloor V_{t}<x_{t}^{\prime}<d_{t}$ or $x_{t}^{\prime}>d_{t}$.

Case 1. If $0<x_{t}^{\prime}<\left\lfloor\frac{d_{t}}{V_{t}}\right\rfloor V_{t}$, then $x_{t}^{\prime}=\left\lfloor\frac{d_{t}}{V_{t}}\right\rfloor V_{t}-\varphi, A_{t}^{\prime}=\left\lfloor\frac{d_{t}}{V_{t}}\right\rfloor-k, q_{t}^{\prime}=0$ by using Property 2.14 and $L_{t}^{\prime}=d_{t}-\left\lfloor\frac{d_{t}}{V_{t}}\right\rfloor V_{t}+\varphi$ with $0<\varphi<\left\lfloor\frac{d_{t}}{V_{t}}\right\rfloor V_{t}, k \in \mathbb{N}$ and $k=\left\lfloor\frac{d_{t}}{V_{t}}\right\rfloor-\left\lceil\left\lfloor\frac{d_{t}}{V_{t}}\right\rfloor-\frac{\varphi}{V_{t}}\right\rceil$. The total cost $\mathscr{C}^{\prime}$ where $\left.x_{t}^{\prime} \in\right] 0 ;\left\lfloor\frac{d_{t}}{V_{t}}\right\rfloor V_{t}[$ is ordered in period $\mathrm{t}$ can be calculated as a function of the total cost $\mathscr{C}^{*(1)}$ where 0 is ordered in period $\mathrm{t}$ and is shown as follows:

$$
\mathscr{C}^{\prime}=\mathscr{C}^{*(1)}+f_{t}-\left(\left\lfloor\frac{d_{t}}{V_{t}}\right\rfloor V_{t}-\varphi\right)\left(l_{t}-p_{t}\right)+a_{t}\left(\left\lfloor\frac{d_{t}}{V_{t}}\right\rfloor-k\right)
$$

If $f_{t}>\left(\left\lfloor\frac{d_{t}}{V_{t}}\right\rfloor V_{t}-\varphi\right)\left(l_{t}-p_{t}\right)-a_{t}\left(\left\lfloor\frac{d_{t}}{V_{t}}\right\rfloor-k\right)$, knowing that $1 \leq \varphi \leq\left\lfloor\frac{d_{t}}{V_{t}}\right\rfloor V_{t}-1$, the solution $x_{t}^{\prime}$ cannot be optimal - a contradiction.

If $f_{t}=\left(\left\lfloor\frac{d_{t}}{V_{t}}\right\rfloor V_{t}-\varphi\right)\left(l_{t}-p_{t}\right)-a_{t}\left(\left\lfloor\frac{d_{t}}{V_{t}}\right\rfloor-k\right)$, then there is another optimal solution that can meet the property.

If $f_{t}<\left(\left\lfloor\frac{d_{t}}{V_{t}}\right\rfloor V_{t}-\varphi\right)\left(l_{t}-p_{t}\right)-a_{t}\left(\left\lfloor\frac{d_{t}}{V_{t}}\right\rfloor-k\right)$, then the total cost $\mathscr{C}^{\prime}$ can be computed as a function of the total cost $\mathscr{C}^{*(2)}$ in which $\left\lfloor\frac{d_{t}}{V_{t}}\right\rfloor V_{t}$ is ordered in period $t$, as follows:

$$
\mathscr{C}^{\prime}=\mathscr{C}^{*(2)}+\varphi\left(l_{t}-p_{t}\right)-k a_{t}
$$

We can detect that $\varphi \geq k V_{t}$. Thus, we have $\varphi\left(l_{t}-p_{t}\right)-k a_{t} \geq 0$ because our problem has to respect the assumption of lost sales. As a result, if $\varphi\left(l_{t}-p_{t}\right)>k a_{t}$ then the solution $x_{t}^{\prime}$ is not optimal - a contradiction. And if $\varphi\left(l_{t}-p_{t}\right)=k a_{t}$ then there is an alternate optimal solution that satisfies the property.

Case 2. If $\left\lfloor\frac{d_{t}}{V_{t}}\right\rfloor V_{t}<x_{t}^{\prime}<d_{t}$, then $x_{t}^{\prime}=\left\lfloor\frac{d_{t}}{V_{t}}\right\rfloor V_{t}+\gamma, A_{t}^{\prime}=\left\lfloor\frac{d_{t}}{V_{t}}\right\rfloor+1, q_{t}^{\prime}=0$ and $L_{t}^{\prime}=d_{t}-\left\lfloor\frac{d_{t}}{V_{t}}\right\rfloor V_{t}-\gamma$ with $0<\gamma<d_{t}-\left\lfloor\frac{d_{t}}{V_{t}}\right\rfloor V_{t}$. One can evaluate the total cost $\mathscr{C}^{\prime}$ where $\left.x_{t}^{\prime} \in\right]\left\lfloor\frac{d_{t}}{V_{t}}\right\rfloor V_{t} ; d_{t}[$ is ordered in period $\mathrm{t}$ as a function of the total cost $\mathscr{C}^{*(2)}$ where $\left\lfloor\frac{d_{t}}{V_{t}}\right\rfloor V_{t}$ is ordered in period $t$, as follows:

$$
\mathscr{C}^{\prime}=\mathscr{C}^{*(2)}+a_{t}-\gamma\left(l_{t}-p_{t}\right)
$$

The cost $\mathscr{C}^{\prime}$ is less than the cost $\mathscr{C}^{*(2)}$ if $a_{t}<\gamma\left(l_{t}-p_{t}\right)$. Otherwise, we can express the total cost $\mathscr{C}^{\prime}$ as a function of the total cost $\mathscr{C}^{*(3)}$ where $d_{t}$ is ordered in period t as follows:

$$
\mathscr{C}^{\prime}=\mathscr{C}^{*(3)}+\left(d_{t}-\left\lfloor\frac{d_{t}}{V_{t}}\right\rfloor V_{t}-\gamma\right)\left(l_{t}-p_{t}\right)
$$

By the assumption of lost sales, if $l_{t}>p_{t}$, then the solution with $\left\lfloor\frac{d_{t}}{V_{t}}\right\rfloor V_{t}<x_{t}^{\prime}<d_{t}$ cannot be optimal - a contradiction, and if $l_{t}=p_{t}$, then, another optimal solution satisfying the property can still be built.

Case 3. If $x_{t}^{\prime}>d_{t}$, then $L_{t}^{\prime}<0$ which contradicts Constraints (2.9) $\left(L_{t} \in \mathbb{R}_{+}\right)$. Thus, $x_{t}^{* 3}=d_{t}$ can be an optimal ordered quantity in period $t$.

We develop an efficient algorithm for the $S P-B_{L S, F T L} R_{1}$ that is based on Properties 2.14 and 2.15. The key idea of this problem is the same for the $S P-B_{L S, O F B} R_{1}$. We have the following costs: $C_{t}^{(1)}, C_{t}^{(2)}, C_{t}^{(3)}, C^{\text {min }}(t)$ and $\mathscr{C}^{*}$ already defined in Section 2.4.1. We refer to Formulas (2.16)-(2.20) by just changing the quantity $x_{t}^{*(3)}$ from $\left\lceil\frac{d_{t}}{V_{t}}\right\rceil V_{t}$ to $d_{t}$ and subsequently the cost $C_{t}^{(3)}$ from $f_{t}+p_{t}\left\lceil\frac{d_{t}}{V_{t}}\right\rceil V_{t}+a_{t}\left\lceil\frac{d_{t}}{V_{t}}\right\rceil-p_{t}^{b}\left(\left\lceil\frac{d_{t}}{V_{t}}\right\rceil V_{t}-d_{t}\right)$ to $f_{t}+p_{t} d_{t}+a_{t}\left\lceil\frac{d_{t}}{V_{t}}\right\rceil$ 


\subsection{4 $\quad$ LSP-BR 1 with lost sales, FTL cost structure and $w>1\left(\right.$ LSP-B $_{L S, F T L} \mathbf{R}_{1}$ with $w>1$ )}

The LSP-B $\mathrm{B}_{L S, F T L} \mathrm{R}_{1}$ with $w>1$ is the most general problem with non-increasing lost sales costs and a constant batch size. We will solve independently, in the same way as before, the

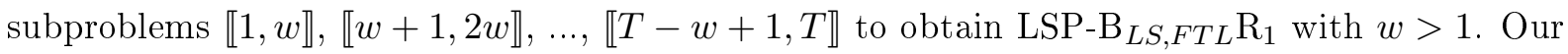
problem will be decomposed into subplans which start and end with zero stock. The optimal cost $C_{w}$ of the subproblem $\llbracket 1, w \rrbracket$ with ordering of full/fractional batches and lost sales can be computed in $O\left(w^{4}\right)$ by a shortest-path like dynamic programming algorithm used in Section 2.3.4, given that all the possible subplan costs can be obtained in $O\left(w^{4}\right)$ time. Thus, we can compute the total optimal cost of LSP-B ${ }_{L S, F T L} \mathrm{R}_{1}$ with $w>1$ with a complexity in $O\left(T w^{3}\right)$ time. The computation of the optimum cost of a subplan relies on the decomposition of $(u, v)$ into different types of cycles. We can find the different forms of replenishment cycles $[t, r]^{u, v(i)}$ for $i=1, \ldots, 8$ and $1 \leq u \leq t<r \leq v \leq w+1$ (see Section 2.3.4) and/or the loss and replenishment cycle $\langle k, v\rangle^{u, v}$ for $1 \leq u \leq k<v \leq w+1$ (see Section 2.4.2).

\section{Properties}

The subproblem $\llbracket 1, w \rrbracket$ possesses several optimality properties presented below. Properties 2.5, 2.6, 2.7, 2.8 (see Section 2.3.4), and 2.13 (see Section 2.4.2) remain valid for the general problem LSP-B ${ }_{L S, F T L} \mathrm{R}_{1}$ with $w>1$.

Property 2.16. There exists an optimal solution, in which, for any subplan $(u, v)$ with $1 \leq u<v \leq w+1$, there is at most either one fractional batch ordered in one of its periods, or one loss period being the period $v-1$.

Proof. Suppose there exists an optimal policy $\pi^{\prime}$ such that in a subplan $(u, v)$, there is both a fractional batch ordered in period i with $u \leq i \leq v-1$ and a loss period located at $v-1\left(L_{v-1}^{\prime}>0\right)$. Define $\xi_{i}$ as the fractional order quantity in period $i\left(0<\xi_{i}<V\right)$ with $\xi_{i}=x_{i}^{\prime}-\left\lfloor\frac{x_{i}}{V}\right\rfloor V$. Let $\epsilon_{1}=\min \left\{V-\xi_{i}, L_{v-1}^{\prime}\right\}$ and $\epsilon_{2}=\min \left\{\xi_{i}, \min _{k=i, \ldots, v-2} s_{k}^{\prime}\right\}$.

We have $\epsilon_{1}>0$ and $\epsilon_{2}>0$, then we can consider 2 other policies:

- policy $\pi^{\prime \prime}$ orders $x_{i}^{\prime}+\epsilon_{1}$ in period $i$, and loses $L_{v-1}^{\prime}-\epsilon_{1}$ in period $v-1$. Policy $\pi^{\prime \prime}$ is feasible.

- policy $\pi^{\prime \prime \prime}$ orders $x_{i}^{\prime}-\epsilon_{2}$ in period $i$, and loses $L_{v-1}^{\prime}+\epsilon_{2}$ in period $v-1$. Policy $\pi^{\prime \prime \prime}$ remains feasible because $\epsilon_{2} \leq \min _{k=i, \ldots, v-2} s_{k}^{\prime}$ and $L_{v-1}^{\prime}+s_{v-2}^{\prime} \leq d_{v-1}$.

Let us compute the total cost of each policy:

$$
\begin{gathered}
\mathscr{C}\left(\pi^{\prime \prime}\right)=\mathscr{C}\left(\pi^{\prime}\right)-\epsilon_{1}\left(l_{v-1}-p_{i}-\sum_{k=i}^{v-2} h_{k}\right) \\
\mathscr{C}\left(\pi^{\prime \prime \prime}\right)=\mathscr{C}\left(\pi^{\prime}\right)+\epsilon_{2}\left(l_{v-1}-p_{i}-\sum_{k=i}^{v-2} h_{k}\right)-a_{i}\left(\left\lceil\frac{x_{i}^{\prime}}{V}\right\rceil-\left\lceil\frac{x_{i}^{\prime}-\epsilon_{2}}{V}\right\rceil\right)
\end{gathered}
$$

Since the policy $\pi^{\prime}$ is optimal, we must have $l_{v-1}-p_{i}-\sum_{k=i}^{v-2} h_{k} \leq 0$ and $l_{v-1}-p_{i}-\sum_{k=i}^{v-2} h_{k} \geq$ $\frac{a_{i}}{\epsilon_{2}}\left(\left\lceil\frac{x_{i}^{\prime}}{V}\right\rceil-\left\lceil\frac{x_{i}^{\prime}-\epsilon_{2}}{V}\right\rceil\right)$. 
Note that the value of $\left\lceil\frac{x_{i}^{\prime}}{V}\right\rceil-\left\lceil\frac{x_{i}^{\prime}-\epsilon_{2}}{V}\right\rceil$ is either 0 or 1 . If it is equal to 0 , then we get $l_{v-1}-$ $p_{i}-\sum_{k=i}^{v-2} h_{k} \geq 0$. As a consequence, it implies that $l_{v-1}-p_{i}-\sum_{k=i}^{v-2} h_{k}=0$, and therefore $\mathscr{C}\left(\pi^{\prime}\right)=\mathscr{C}\left(\pi^{\prime \prime}\right)=\mathscr{C}\left(\pi^{\prime \prime \prime}\right)$. Thus, both policies are also optimal, verifying Property 2.16 . If the value of $\left\lceil\frac{x_{i}^{\prime}}{V}\right\rceil-\left\lceil\frac{x_{i}^{\prime}-\epsilon_{2}}{V}\right\rceil$ is 1 , then we obtain $l_{v-1}-p_{i}-\sum_{k=i}^{v-2} h_{k}>0$. This is a contradiction to the optimality of $\pi^{\prime}$ and the policy $\pi^{\prime \prime}$ becomes optimal $\left(\mathscr{C}\left(\pi^{\prime \prime}\right)<\mathscr{C}\left(\pi^{\prime}\right)\right)$.

Hence, if there is a positive lost sales quantity, then all ordering periods are large. Now, we can directly use Property 2.12 and Corollary 2.1.

\section{How to compute $\mathscr{C}(u, v)$}

All these properties imply that the cost of the subplan $(u, v)$ for $1 \leq u<v \leq w+1$ in LSP$\mathrm{B}_{L S, F T L} \mathrm{R}_{1}$ with $w>1$ is the minimum between the cost $\mathscr{C}(u, v)$ in LSP-B $\mathrm{B}_{F T L} \mathrm{R}_{1}$ with $w>1$,

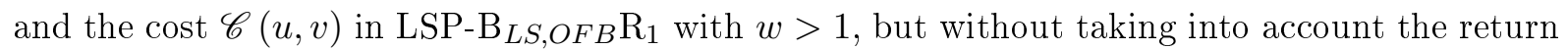
revenue since the retailer can order fractional batches. Hence, the running time to compute all the values of $\mathscr{C}(u, v)$ is bounded by $O\left(w^{4}\right)$. They are obtained as follows:

$$
\begin{aligned}
& \mathscr{C}(u, v)=\min \left\{\min _{1 \leq i \leq 6}\left\{\mathscr{C}_{i}(u, v)\right\} ;\right. \\
& \min _{u \leq t \leq v-1 \leq w}\left\{G_{t}^{u, v}+\min \left\{\mathbb{1}_{D_{t, v-1}-s_{t-1}^{u, v} \geq L_{v-1}^{u, v}} f_{t}+\left(p_{t}+\frac{a_{t}}{V}\right)\left\lfloor\frac{D_{t, v-1}-s_{t-1}^{u, v}}{V}\right\rfloor V\right.\right. \\
& +H_{t}^{u, v}+l_{v-1} L_{v-1}^{u, v} ; \\
& \left.\left.\left.\mathbb{1}_{t=v-1} \mathbb{1}_{d_{v-1}-s_{v-2}^{u, v} \geq L_{v-1}^{u, v}} l_{v-1}\left|d_{v-1}-s_{v-2}^{u, v}\right|\right\}\right\}\right\}
\end{aligned}
$$

Once all these values are predetermined, the value of $C_{w}$ can be obtained in $O\left(w^{4}\right)$ time by using (2.11). Hence, the LSP-B ${ }_{L S, F T L} \mathrm{R}_{1}$ with $w>1$ is solved in $O\left(T w^{3}\right)$ time.

\section{Numerical example for LSP-B L $_{L S T L} \mathbf{R}_{1}$ with $w>1$}

We consider the same example presented for the algorithm of LSP-B ${ }_{L S, O F B} \mathrm{R}_{1}$ with $w>1$ (see Section 2.4.2) by ordering in full and/or fractional batches. The optimal decomposition of subplans is $(1,4)$ and $(4,5)$. The optimal cost of $(1,4)$ is the cost of the cycle $\langle 1,4\rangle^{1,4}$ as in the example of Section 2.4.2. In $(4,5)$, there is no return and the period 4 is a large and fractional ordering period. Its optimal cost is the cost of the cycle $\mathscr{L}[4,5]^{4,5(1)}$ as in the example of Section 2.3.4.

\subsection{Computational experiments}

In this section, for each problem of LSP-BR 1 with $w>1$, we coded the MILP in Xpress Mosel version 3.10 with default parameters for cut strategy and presolving, and we implemented the dynamic programming algorithm (DP) in Java in order to compare the computational time of both resolution methods under different parameters. For the MILP, we used for all problems, aggregate formulations. All the tests were executed on an Intel Core $2.40 \mathrm{GHz}, 8 \mathrm{~GB}$ RAM. The resolution time of the two methods that appears in Table 2.7 for the problems with $w>1$ is the average of 10 instances generated in the corresponding intervals.

The execution time of MILP depends on all the problem parameters: length of the horizon, periodicity of the return periods, demand, batch size, and cost and revenue parameters. Hence, 
its computational time is unpredictable. In contrast, the complexity of DP is only dependent on the length of the horizon, thus its execution time can be easily predictable.

We consider six different types of instances. We performed the tests for $T=20, T=50$ and $T=100$. The periodicity of the return periods is fixed to $T$, which means that there is one return over the planning horizon. We chose two configurations for the demands which follow a Uniform distribution with a stationary batch size: $D 1=U(5,20)$ with $V 1=4$ and $D 2=U(30,100)$ with $V 2=10$.

Concerning the cost and revenue parameters, we consider the setup cost uniformly distributed in the interval $U(50,100)$ in each period. The unit procurement costs and the fixed costs per batch replenished are calculated as a function of the setup cost, $p_{t}=0.01 f_{t}$, and $a_{t}=0.1 f_{t}$. We have only one return revenue since we have considered $w=T$, it is computed as a function of the maximum of all the unit procurement costs, $p_{w}^{b}=0.4 \max _{t=1, \ldots, T}\left\{p_{t}\right\}$, in order to respect the assumption of the form $\mathrm{R}_{1}$. The inventory holding costs follow the Uniform distribution with the minimum value 0.05 and the maximum value 2 . For the $\mathrm{LSP}_{-} \mathrm{BR}_{1}$ with lost sales, we consider a stationary lost sales cost, $l_{t}=l=1.7 \max _{t=1, \ldots, T}\left\{p_{t}+\frac{a_{t}}{V}\right\}$ to satisfy the lost sales assumption.

We analyze the performance of the proposed methods in terms of resolution time. The test results are reported in Table 2.7 for the four cases of LSP-BR 1 with $w>1$ knowing that we limit the running time of Xpress to 300 seconds and we give its gap if we do not find the optimal solution after 300 seconds. Note that the gap provided by Xpress is computed according to the best integer solution and the best lower bound found until 300 seconds.

Table 2.7: Computational results for DP and MILP of $\mathrm{LSP}_{-\mathrm{BR}_{1}}$ with $w=T(w>1)$

\begin{tabular}{|c|c|c|c|c|c|c|c|}
\hline & & \multicolumn{2}{|c|}{$T=20$} & \multicolumn{2}{|c|}{$T=50$} & \multicolumn{2}{|c|}{$T=100$} \\
\hline & & $D 1$ & $D 2$ & $D 1$ & $D 2$ & $D 1$ & D2 \\
\hline \multirow{4}{*}{ DP time (seconds) } & $\mathrm{LSP} \mathrm{B}_{O F B} \mathrm{R}_{1}$ with $w>1$ & 0 & 0 & 0 & 0 & 0.01 & 0.01 \\
\hline & LSP-B $_{F T L} \mathrm{R}_{1}$ with $w>1$ & 0.01 & 0.07 & 2.58 & 2.13 & 208.74 & 208.08 \\
\hline & LSP-B $_{L S, O F B} \mathrm{R}_{1}$ with $w>1$ & 0.02 & 0.01 & 0.25 & 0.17 & 1.56 & 1.56 \\
\hline & LSP-B $_{L S, F T L} \mathrm{R}_{1}$ with $w>1$ & 0.12 & 0.09 & 2.36 & 2.04 & 243.20 & 251.61 \\
\hline \multirow{4}{*}{ MILP time (seconds) } & LSP-B ${ }_{O F B} \mathrm{R}_{1}$ with $w>1$ & 0.21 & 0.43 & 4.84 & 96.11 & $>300$ & $>300$ \\
\hline & LSP-B $_{F T L} \mathrm{R}_{1}$ with $w>1$ & 0.15 & 0.08 & 0.47 & 0.32 & 2.81 & 4.07 \\
\hline & LSP-B $_{L S, O F B} \mathrm{R}_{1}$ with $w>1$ & 0.10 & 0.07 & 0.16 & 0.30 & 0.62 & 1.16 \\
\hline & LSP-B $_{L S, F T L} \mathrm{R}_{1}$ with $w>1$ & 0.23 & 0.07 & 0.32 & 0.30 & 2.65 & 2.34 \\
\hline \multirow{4}{*}{$\begin{array}{l}\text { MILP gap after } 300 \\
\text { seconds (percent) }\end{array}$} & LSP-B ${ }_{O F B} \mathrm{R}_{1}$ with $w>1$ & 0 & 0 & 0 & 0 & $0.23 \%$ & $1.48 \%$ \\
\hline & LSP-B $F T L \mathrm{R}_{1}$ with $w>1$ & 0 & 0 & 0 & 0 & 0 & 0 \\
\hline & LSP-B $_{L S, O F B} \mathrm{R}_{1}$ with $w>1$ & 0 & 0 & 0 & 0 & 0 & 0 \\
\hline & LSP-B $_{L S, F T L} \mathrm{R}_{1}$ with $w>1$ & 0 & 0 & 0 & 0 & 0 & 0 \\
\hline
\end{tabular}

When the number of periods is less than 50, DP can be advantageous to use for the four problems, because it does not depend on problem parameters. For $T=100$, the execution times of LSP-B ${ }_{F T L} \mathrm{R}_{1}$ and $\mathrm{LSP}-\mathrm{B}_{L S, F T L} \mathrm{R}_{1}$ remain almost 4 minutes. Using MILP, the optimal solution in each problem, in most tested instances, is obtained quite instantaneously, except for the following instances: $T=50$ and $D 2, T=100$ and $D 1$, and $T=100$ and $D 2$ in LSP-B ${ }_{O F B} \mathrm{R}_{1}$. The behavior of MILP remains unpredictable and depends on many parameters because, for

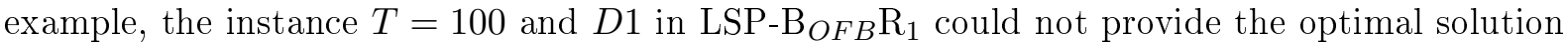

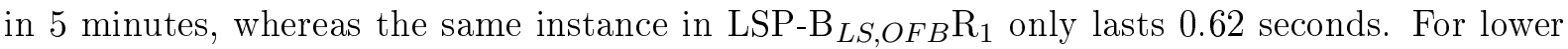
values of demands, most of the MILP time provides the optimal solution very quickly in all problems. 


\subsection{Conclusion}

In this chapter, we considered the single-item lot sizing problem with batch ordering under the buyback contract. In our model, we merged the buyback contract into the LSP by considering that the retailer returns the unused units to the supplier either at the end of each period $t$, denoted by $w=1$ or at the end of every $w$ periods $w>1$ with full return and partial refund. Another option we studied is the possibility to not satisfy all the demands, knowing that losing a batch costs more than purchasing it. Therefore, for both LSP-BR 1 with cyclic returns, without and with lost sales, we tackled four problems by assuming different hypotheses. In the first problem, we only consider full batches with $w=1$. The second problem is with OFB and $w>1$. To obtain the two problems that remain, we changed the OFB cost structure in the first two problems to the FTL cost structure.

We used some dominance properties existing in the literature, we introduced others, in order to propose exact resolution methods for different extensions of LSP-BR 1 with cyclic returns, and we performed computational tests by varying different parameters, to compare the performance of the resolution methods. We detected LSP under buyback contract with batch ordering and lost sales not studied in the literature and we proposed polynomial time algorithms to solve them. The buyback assumption of form $R_{1}$ does not make the problem difficult because the return periods are known. On the contrary, it allows for decomposing the problem into smaller subproblems. However, the lost sales assumption can increase the overall complexity of the problem by increasing the possible production plans. Table 2.8 shows different cases studied in this chapter with the respective computational complexities.

Table 2.8: Complexities of the algorithms for different cases studied in Chapter 2

\begin{tabular}{|c|c|c|c|c|c|c|c|c|}
\hline \multirow{3}{*}{ Hypotheses } & \multicolumn{4}{|c|}{ Without lost sales } & \multicolumn{4}{|c|}{ With lost sales } \\
\hline & \multicolumn{2}{|c|}{ OFB } & \multicolumn{2}{|c|}{ FTL } & \multicolumn{2}{|c|}{ OFB } & \multicolumn{2}{|c|}{ FTL } \\
\hline & $\begin{array}{l}w=1 \\
\text { with } V_{t}\end{array}$ & $\begin{array}{l}w>1 \\
\text { with } V\end{array}$ & $\begin{array}{l}w=1 \\
\text { with } V_{t}\end{array}$ & $\begin{array}{l}w>1 \\
\text { with } V\end{array}$ & $\begin{array}{l}w=1 \\
\text { with } V_{t}\end{array}$ & $\begin{array}{l}w>1 \\
\text { with } V\end{array}$ & $\begin{array}{l}w=1 \\
\text { with } V_{t}\end{array}$ & $\begin{array}{l}w>1 \\
\text { with } V\end{array}$ \\
\hline Complexity & $O(T)$ & $O(T w)$ & $O(T)$ & $O\left(T w^{3}\right)$ & $O(T)$ & $O\left(T w^{3}\right)$ & $O(T)$ & $O\left(T w^{3}\right)$ \\
\hline
\end{tabular}

In the next chapter of this thesis, we extend this problem by adding a percentage $\rho$ of return and the concept of disposal. Besides, we study other forms of the buyback contract: $R_{2}$ which imposes a time limit on returns and $R_{3}$ which fixes returns in procurement periods. For all the problems with $\rho=1$, we propose polynomial algorithms but for the case $\rho<1$, we develop a polynomial algorithm for the form $R_{1}$ and concerning the types $R_{2}$ and $R_{3}$ we prove that these problems could be solved using the algorithm proposed for the resource constrained shortest path problem with lower and upper limits (Beasley and Christofides, 1989). 


\section{Chapter 3}

\section{Full batch replenishment with buyback and disposal options}

In this chapter, we study several extensions of the single-item lot sizing problem with only full batch replenishment under the buyback contract. We consider three forms of the buyback contract in a system with one retailer and one supplier. In the first form, the return periods are fixed. In the second form, the retailer can only return units to the supplier in the first $j$ periods. Finally, in the third form, the retailer may return products only in replenishment periods. Each contract is characterized by the maximum return percentage which can be either $100 \%$ (full return) or less than $100 \%$ (partial return). We also consider the disposal concept to get rid of the units which cannot be stored nor returned to the supplier because of the trade-off between low return revenues and high holding costs.

For each extension of LSP-B with buyback and disposal options, we propose a mathematical model and dominance properties. We develop efficient polynomial time algorithms for some of the above problems, followed by experimental results, and show that the remaining ones can be solved by the algorithm based upon a Lagrangian relaxation, proposed for the resource constrained shortest path problem with double sided inequality constraints.

A part of this work is presented in ROADEF Lorient (2018), see Farhat et al. (2018c). 


\subsection{Introduction}

This work presents the single item uncapacitated LSP considering a buyback contract over a planning horizon of $T$ periods. The system considered is composed of a retailer replenishing one type of product from a supplier. At a given period, purchased units are delivered to the retailer by only full batches of a constant size from the supplier, in order to satisfy the deterministic demand without backlogging nor lost sales. At some periods, indicated by the buyback contract type, the retailer can return a certain amount of the unused items to the supplier with a revenue and can dispose of the remaining ones with no cost nor revenue. We denote the general problem by LSP-BD-R (B for batch ordering, D for disposal option and R for return).

We study three types of buyback contracts: one with fixed return periods $\left(\mathrm{R}_{1}\right)$, the other one with a time limit on the return periods $\left(\mathrm{R}_{2}\right)$ and the last one with returns allowed only in procurement periods $\left(\mathrm{R}_{3}\right)$. Refer to Chapter 1 , Subsection 1.4.1 in order to see the different forms of the buyback contract. Each type represents many features. The first feature depends on the nature of return: fixed, which means that there are known return periods, or variable, with unknown return periods. The second feature is the constraint of buyback contract which links cumulatively the purchased quantity $x$ and the returned quantity $q$. The third feature is the return percentage $\rho$. There is either a generous policy which allows for a full return $(\rho=1)$, or an avaricious policy which offers a partial return $(\rho<1)$. We allow the refund amount $p^{b}$ to be strictly less than the procurement cost $p$ (partial refund). The difference $p-p^{b}$ for the supplier can be interpreted as a restocking fee or a nonrefundable charge. The proposed algorithms in this study can also be applied to solve the problems under a contract with full buyback refund.

Another consideration of this study is the assumption of disposals. In a general setting, the disposal represents either a revenue or a cost for a retailer. In our work, we assume that the disposal has neither a revenue nor a cost because we consider that the retailer decides to give the unused and unreturned quantities to charities by asking these organizations to come and to get them, especially when the batch size is significantly large.

Therefore, the objective is to determine the optimal procurement planning strategy in order to satisfy the retailer demand while minimizing the total ordering and storage costs taking into account the return revenues, and respecting buyback constraints and disposals. For these different extensions of the LSP with only full batch ordering and disposals under different types of buyback contract, we establish efficient algorithms for certain extensions and prove the similarity between the other extensions and the resource constrained shortest path problem (RCSPP).

The structure of Chapter 3 is organized as follows. Section 3.2 provides a description of the general LSP-BD-R studied in this work. Section 3.3 is dedicated to the different cases of LSP-BD-R $\mathrm{R}_{1}$ with fixed return periods in which we formulate the general problem as a mixed integer linear program and we propose exact polynomial time algorithms for two extensions. Section 3.4 presents a mathematical formulation for the LSP-BD- $R_{2}$ with time limit on returns by constructing a dynamic programming algorithm for the case with $\rho=1$ and, by proving that the case with $\rho<1$ is similar to RCSPP. Section 3.5 develops dominance properties and a solution method for the LSP-BD-R $\mathrm{R}_{3}$ with return possibility allowed only in procurement periods. Section 3.6 presents the numerical results performed in this chapter. Section 3.7 draws a conclusion of the chapter. 


\subsection{Problem description}

The problem consists in planning a single-item procurement of a retailer having signed a buyback contract with a supplier, which can take several forms. The aim is to satisfy the deterministic demand $d_{t}$ using only full batch replenishment over a finite time horizon $1, \ldots, T$. The quantity $x_{t}$ ordered from the supplier is shipped in $A_{t}$ batches in period $t$, and incurs a fixed setup cost $f_{t}$ and a fixed cost per batch $a_{t}$, in addition to a unit procurement cost $p_{t}$. The binary setup variable $y_{t}$ is fixed to 1 when a positive quantity is ordered in $t$, then we have $x_{t} \leq\left\lceil\frac{\sum_{j=t}^{T} d_{j}}{V}\right\rceil V y_{t}$, for all $t \in\{1, \ldots, T\}$. We denote by $V$ the size of the replenished batches. Notice that in order to derive polynomial time algorithms, we consider the batch size to be stationary over time. Moreover, we assume $x_{t}=A_{t} V$ to purchase a multiple of the batch size $V$. No fractional batches are accepted.

The inventory level at the retailer from period $t$ to $t+1$ is denoted by $s_{t}$. Keeping one unit of item in stock incurs a holding cost of $h_{t}$. We assume, without loss of generality, a null stock level at the beginning of the planning horizon. Otherwise, this quantity can be retrieved from the demand of the first periods, till obtaining null entering inventory.

With the general buyback contract, we consider that the supplier can buy back, at the end of each period $t$, an unsold inventory quantity $q_{t}$ with a maximum return percentage of $\rho$ such that $0 \leq \rho \leq 1$, by paying the retailer a pay-back price of $p_{t}^{b}$ per unit. In order to avoid the speculation for the retailer, we assume that this revenue $p_{t}^{b}$ is less than all the unit procurement costs $p_{1}, p_{2}, \ldots, p_{T}$. We consider fixed or variable return periods denoted by $w_{i}$ for $i \in \mathbb{N}^{*}$ and $1 \leq i \leq T$ depending on the type of the contract considered.

This contract owns different structures used in real life to cope with the demand supply problem over a finite horizon of $T$ periods. We consider three types of the buyback contract in which we study the full and partial return policies.

(i) Type $\mathrm{R}_{1}$ : a buyback contract with fixed return periods between two members of the supply chain. They can be cyclic (with $w$ being the periodicity of return) or acyclic (with $w_{i}$ being the return periods).

(ii) Type $\mathrm{R}_{2}$ : a contract with a time limit on returns in which the supplier allows the retailer to return, just over the first $j$ periods, a maximum of $\rho \sum_{t=1}^{j} x_{t}$ units. In the remaining periods, no returns are accepted. The return periods are thus decision variables.

(iii) Type $\mathrm{R}_{3}$ : the retailer can return units only in a procurement period $t$. The return periods are thus decision variables.

For the two types $\mathrm{R}_{1}$ and $\mathrm{R}_{2}$, a return period may not coincide with an ordering period in which the supplier replenishes the retailer. Thus, we suppose that the transportation cost of the returned quantity $q_{t}$ from the retailer to the supplier at the end of period $t$ is deduced from the initial return revenue. We get a reduced return revenue that we assume to be positive in order to encourage the retailer to sign with the supplier a buyback contract, otherwise, it entails additional costs. For the last type $R_{3}$, we use the initial return revenue because the supplier takes charge of the transportation cost of the returned quantity in the same procurement period.

In this study, we also consider the disposal option. In certain cases where the supplier offers the retailer a full return but with very low buyback revenues compared to higher inventory costs, or when the supplier requires the retailer to return a limited number of the unsold items, we assume that the retailer can decide to get rid of the remaining items. This is due to the fact that 
the unsold and unreturned inventory can be quite costly. Thus, we introduce $e_{t}$ designating the discarded quantity in period $t$ with null disposal costs nor revenues.

In the next sections, we will study different extensions of this problem formulated as a mixed integer linear program (MILP) dealing with different buyback constraints according to three return policies. The objective is to propose an optimal procurement plan with only full batches which satisfies demand without backlogging nor lost sales, while minimizing the total procurement and inventory costs, taking into account total return revenues and the disposal option.

\section{Parameters}

$\boldsymbol{T}$ : number of periods in the planning horizon,

$f_{t}$ : setup cost in period $t$,

$p_{t}$ : unit procurement cost in period $t$,

$a_{t}$ : fixed cost per batch in period $t$,

$V$ : batch size,

$h_{t}$ : unit holding cost at the end of period $t$,

$\rho$ : maximum return percentage,

$p_{t}^{b}$ : unit return revenue in period $t$

$\boldsymbol{d}_{t}$ : demand in period $t$.

$\boldsymbol{w}_{i}$ : period of $i^{t h}$ return, and $\boldsymbol{N}$ : total number of returns over the horizon 1..T ( $w_{i}$ becomes a decision variable in LSP-BD-R 2 and LSP-BD-R 3 ).

\section{Other definitions}

$\boldsymbol{D}_{i, j}$ : cumulative demand from period $i$ to period $j$.

$(\boldsymbol{u}, \boldsymbol{v})$ : a subplan with $1 \leq u<v \leq T+1$, the periods $u$ and $v$ are consecutive periods having null entering stock levels $\left(s_{u-1}=s_{v-1}=0\right)$, and, in each period in the subplan, we have $s_{t}>0$ for $t=u, \ldots, v-2$.

$\boldsymbol{R}_{u, j}$ (Li et al. (2004)): ending inventory level of period $j$ in a subplan $(u, v)$ if we acquire the minimal number of batches $\left\lceil\frac{D_{u, j}}{V}\right\rceil$ satisfying the demands $d_{u}, d_{2}, \ldots, d_{j}$ without return nor disposal.

$$
R_{u, j}=\left\lceil\frac{D_{u, j}}{V}\right\rceil V-D_{u, j} \quad \forall j \in\{u, \ldots, v-1\} \text { with } R_{u, u-1}=0
$$

\section{Decision variables}

$x_{t}$ : procurement quantity in period $t$,

$\boldsymbol{y}_{\boldsymbol{t}}$ : binary setup variable in period $t$,

$\boldsymbol{A}_{\boldsymbol{t}}$ : number of batches replenished in period $t$,

$s_{t}$ : inventory level at the end of period $t$,

$\boldsymbol{q}_{t}$ : returned quantity of unsold items at the end of period $t$,

$\boldsymbol{e}_{t}$ : discarded quantity of unused items at the end of period $t$.

$\boldsymbol{q}_{t}^{u, v}$ : returned quantity at the end of period $t$ in a subplan $(u, v), \boldsymbol{e}_{t}^{u, v}$ : discarded quantity at the end of period $t$ in a subplan $(u, v)$. 


\subsection{LSP with batch ordering, disposal and fixed return periods $($ LSP-BD-R 1 )}

The structure of the buyback contract considered in this section mainly concerns the perishable products (pharmaceuticals, baked products) and products with short-term life (computer hardware and software). We consider that the unused products can be returned back to the supplier at the end of given periods $w_{i}$ with $1 \leq w_{i} \leq T, i=1, \ldots, N$, with $N$ being the total number of return periods in the planning horizon. We assume, without loss of generality, that $w_{N}=T$. The parameters $w_{i}$ and $N$ are determined by both the supplier and the retailer, within the contract in accordance with the lifetime of the products and the costs of carrying inventory in both sides. The return periods $w_{i}$ may or may not be cyclic. At the end of known return periods $w_{i}$, the retailer has always zero stock: the unused products are returned to the supplier and/or they are discarded. Hence, there are $N$ independent problems to optimize related to each subproblem $\llbracket w_{i-1}+1, w_{i} \rrbracket$ knowing that $i=1, \ldots, N$ and $w_{0}=0$ (See Figure 3.1). The total cost of the LSP-BD- $\mathrm{R}_{1}$ is computed as the sum of the costs of these independent subproblems.

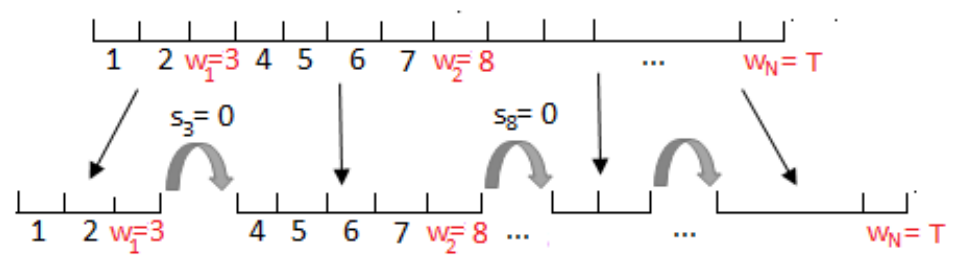

Figure 3.1: $N$ independent subproblems of the general LSP-BD-R

At the end of a given period $w_{i}$, the supplier allows the retailer to return the quantity $q_{w_{i}}$ which is limited by a percentage of the total quantity replenished within the interval $\llbracket w_{i-1}+1, w_{i} \rrbracket$, thus it is equivalent to $\rho \sum_{k=w_{i-1}+1}^{w_{i}} x_{k}$ units. For each returned unit, a unit return revenue $p_{w_{i}}^{b}$ is earned, for all $i=1, \ldots, N$. We consider $p_{t}^{b}=0, \forall t \in\left\{w_{i-1}+1, \ldots, w_{i}-1\right\}$ given that no returns are allowed between the periods $w_{i-1}+1$ and $w_{i}-1$. We also consider $p_{w_{i}}^{b}<p_{t}$, $\forall t \in\left\{w_{i-1}+1, \ldots, w_{i}\right\}, \forall i \in\{1, \ldots, N\}$ to protect the supplier from the speculation. In the case where $\rho=1$, the retailer has the possibility to return all the unsold units if it is profitable. Otherwise, in case of $(\rho<1)$, the latter can only return a limited quantity to the supplier depending on the value of $\rho$. In these two cases, the retailer can dispose of the quantity $e_{t}$ in each period $t$. Note that at the end of each period $w_{i}$, the stock level drops to 0 .

The objective is to minimize the total cost over the whole planning horizon while respecting all the given constraints:

$$
\min \sum_{t=1}^{T}\left(f_{t} y_{t}+p_{t} x_{t}+a_{t} A_{t}+h_{t} s_{t}-p_{t}^{b} q_{t}\right)
$$

Material balance equations:

$$
\begin{aligned}
& x_{t}+s_{t-1}=d_{t}+s_{t}+q_{t}+e_{t} \\
& s_{0}=0
\end{aligned} \quad \forall t=1, \ldots, T
$$


Conditions of the buyback contract type $\mathrm{R}_{1}$ :

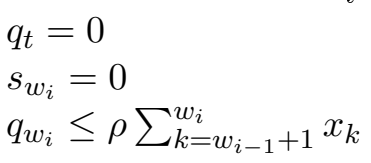

$$
\begin{aligned}
& \forall t=1, \ldots, T \text { such that } t \neq w_{i}, i=1, \ldots, N \\
& \forall i=1, \ldots, N \\
& 0 \leq \rho \leq 1, \forall i=1, \ldots, N
\end{aligned}
$$

Setup variable generation constraints:

$$
x_{t} \leq\left\lceil\frac{\sum_{j=t}^{T} d_{j}}{V_{t}}\right\rceil V y_{t} \quad \forall t=1, \ldots, T
$$

Only full batch replenishment constraints:

$x_{t}=A_{t} V$

$$
\forall t=1, \ldots, T
$$

Nonnegativity and binary constraints:

$$
\begin{array}{ll}
x_{t}, s_{t}, q_{t}, e_{t} \in \mathbb{R}_{+} & \forall t=1, \ldots, T \\
A_{t} \in \mathbb{N}, y_{t} \in\{0,1\} & \forall t=1, \ldots, T
\end{array}
$$

In the following, we solve each of the $N$ subproblems as $\llbracket 1, W_{i} \rrbracket$ for $i=1, \ldots, N$, with $W_{i}=w_{i}-w_{i-1}$. The total cost will be constituted of the individual cost of each subproblem. We study the two sub-cases, namely: LSP-BD-R $1, \rho=1$ with full return and LSP-BD-R $\mathrm{R}_{1, \rho<1}$ with partial return.

\subsubsection{LSP-BD- $\mathbf{R}_{1}$ with full return (LSP-BD-R $\mathbf{R}_{1, \rho=1}$ )}

The LSP-BD- $\mathrm{R}_{1, \rho=1}$ is composed of $N$ independent problems $\left(\llbracket 1, W_{i} \rrbracket\right.$ for $\left.i \in\{1, \ldots, N\}\right)$ to be separately solved. Throughout $\llbracket 1, W_{i} \rrbracket$, the retailer can get rid of the unreturned and unsold quantity in each period and the supplier allows only one return of all the left-over items $(\rho=1)$ at the end of period $W_{i}$, without lost sales. We first state optimality properties and algorithms for the general case with linear holding costs, and at the end we present the idea of complexity reduction for the special case with $p_{W_{i}}^{b} \geq \sum_{k=1}^{W_{i}-1} h_{k}$ since there is one return placed in the last period of $\llbracket 1, W_{i} \rrbracket$.

\section{LSP-BD-R $\mathbf{R}_{1, \rho=1}$ for the general case}

The main idea of the algorithm is to detect the periods and quantities of return and of disposal in each possible subplan $(u, v)$ in order to satisfy the demand in periods $u, \ldots, v-1$, for $1 \leq u<v \leq W_{i}+1$ at minimum cost, and thereafter, to compute the total optimal cost over 1..T using a shortest path algorithm. The use of a shortest path algorithm to compute the total cost is a quite common technique once the cost of individual subplan $(u, v)$ are all precomputed. The interesting part remains the efficient computation of the cost of each subplan.

\section{Some preliminaries and properties:}

Property 3.1. There is an optimal solution, in which, for any subplan $(u, v)$ with $1 \leq u<v \leq$ $W_{i}+1$, there exists at most one disposal period situated in the first period of $(u, v)$, i.e., period $u$. In other words, in an optimal policy, we have $s_{t-1} e_{t}=0, \forall t=1, \ldots, W_{i}$.

Proof. First, let us prove that there is at most one disposal period in a subplan. We suppose, that in a subplan $(u, v)$ such that $1 \leq u<v \leq W_{i}+1$, we have two disposal periods $i$ and $j$ $\left(e_{i}^{u, v}>0\right.$ and $\left.e_{j}^{u, v}>0\right)$ with $u \leq i<j \leq v-1$. Let $\alpha_{i, j}=\min \left\{e_{j}^{u, v}, \min _{k=i, \ldots, j-1} s_{k}\right\}>0$. 
Increasing $e_{i}^{u, v}$ by $\alpha_{i, j}$ and decreasing both $e_{j}^{u, v}$ and the inventory levels $s_{k}(k=i, \ldots, j-1)$ by $\alpha_{i, j}$, we obtain another feasible solution. With this modification, if $\alpha_{i, j}=e_{j}^{u, v}$, then the period $i$ becomes the only disposal period in $(u, v)$. If $\alpha_{i, j}=\min _{k=i, \ldots, j-1} s_{k}$, then, we obtain two subplans with each having one disposal period: in the first one, the disposal period corresponds to $i$ and in the second one to $j$. Moreover, it is easy to verify that the total cost of this new solution will be decreased by the amount of $\alpha_{i, j} \sum_{k=i}^{j-1} h_{k}$. Hence, we obtain an optimal solution satisfying the fact that there is at most one disposal period in a subplan.

Second, let us prove that, if it exists, the disposal period is the first period $u$ of the subplan $(u, v)$. We consider now an optimal policy $\pi^{\prime}$ in which there is a subplan $(u, v)$ whose disposal period is $j$ with $j>u$.

We will modify the policy $\pi^{\prime}$ in the same way as above (we change period $i$ by period $u$ knowing that in $\pi^{\prime}, e_{u}^{u, v}=0$ ). Thus, in the modified policy, we decide to get rid of $\alpha_{u, j}$ units at the period $u$, to store $s_{k}-\alpha_{u, j}$ units from $u$ to $j-1$ by leaving the inventory quantities on the other periods $(j, \ldots, v-1)$ unchanged, and to dispose of $e_{j}^{u, v}-\alpha_{u, j}$ units in period $j$. The total cost of this new policy is lower than the one of $\pi^{\prime}$.

If $\alpha_{u, j}=e_{j}^{u, v}$, then $(u, v)$ is a subplan in which $u$ is the only disposal period.

If $\alpha_{u, j}=\min _{k=u, \ldots, j-1} s_{k}=s_{b}$ with $u \leq b \leq j-1$, then the new stock in period $b$ drops to 0 and therefore $(u, v)$ is divided into at least 2 subplans: $(u, b+1)$ and $(b+1, v)$. The subplan $(u, b+1)$ has one disposal period situated in period $u$. However, in the subplan $(b+1, v)$, the disposal period is placed in $j$.

Now, we will prove that either $(b+1, v)$ is the unique subplan in which $b+1$ is the disposal period or $(b+1, v)$ is divided into several subplans such that the disposal period of each subplan can only be the first period. We apply iteratively the same procedure performed in the second part of this proof. We take into account the updated quantities of $(b+1, v)$ during the modification of the $(u, v)$ solution.

The more the disposal period $j$ is placed at the beginning of the optimal subplan, the lower will be the inventory costs.

Property 3.2. In an optimal policy, for a $\operatorname{subplan}(u, v)$ with $1 \leq u<v \leq W_{i}$, there is at most one disposal period and no return. Returns are only allowed for the subplans $\left(u, W_{i}+1\right)$ in period $W_{i}$. We can easily compute the disposal quantity $e_{u}^{u, v}$ with $e_{u}^{u, v}=\left\lceil\frac{D_{u, v-1}}{V}\right\rceil V-D_{u, v-1}$. Note that $e_{u}^{u, v}<V$.

Proof. If $1 \leq u<v \leq W_{i}$, then, the retailer cannot return between $u$ and $v-1$ and according to Property 3.1 , there is at most one disposal in $(u, v)$. The retailer has to buy only $\left\lceil\frac{D_{u, v-1}}{V}\right\rceil$ batches which totally satisfy the demand of the subplan $(u, v)$ because it is not profitable for him to procure extra batches just to throw away them with a zero disposal revenue. Hence, the remaining units $\left\lceil\frac{D_{u, v-1}}{V}\right\rceil V-D_{u, v-1}$ are disposed of in period $u$ to have a lower total cost.

Property 3.3. There exists an optimal policy such that, in a subplan $\left(u, W_{i}+1\right)$, there is at most either one disposal period being the period $u$, or one return period being the period $W_{i}$. Thus, we have $q_{W_{i}}^{u, W_{i}+1} e_{u}^{u, W_{i}+1}=0$. If such a disposal period exists, then, we can compute the quantity disposed by $e_{u}^{u, W_{i}+1}=\left\lceil\frac{D_{u, W_{i}}}{V}\right\rceil V-D_{u, W_{i}}$. Otherwise, the return quantity is computed as $q_{W_{i}}^{u, W_{i}+1}=\left\lceil\frac{D_{u, W_{i}}}{V}\right\rceil V-D_{u, W_{i}}$.

Proof. For the subplans $(u, v)$ with $1 \leq u<v=W_{i}+1$, suppose that there exists, in an optimal solution, a subplan $\left(u, W_{i}+1\right)$ with $e_{u}^{u, W_{i}+1}>0$ and $q_{W_{i}}^{u, W_{i}+1}>0$. In this subplan, the retailer 
procures a number of batches which only satisfies $d_{u}, d_{u+1}, \ldots, d_{W_{i}}$, on the one hand because he has a null disposal revenue, and on the other hand, he has a non-negative return revenue $p_{W_{i}}^{b}$ being less than all the unit procurement $\operatorname{costs} p_{t}$, for all $t=1, \ldots, W_{i}$. Without this relationship between the procurement cost and the non-negative return revenue, the retailer can buy an unlimited quantity in $\left(u, W_{i}+1\right)$ and resell it to the supplier to obtain an unbounded profit, which is an unfeasible case. Hence, we have $e_{u}^{u, W_{i}+1}+q_{W_{i}}^{u, W_{i}+1}=\left\lceil\frac{D_{u, W_{i}}}{V}\right\rceil V-D_{u, W_{i}}<V$.

First, we decrease $e_{u}^{u, W_{i}+1}$ by $e_{u}^{u, W_{i}+1}$ and we increase both the quantities remaining in stock $s_{k}$ $\left(u \leq k \leq W_{i}-1\right)$ and $q_{W_{i}}^{u, W_{i}+1}$ by $e_{u}^{u, W_{i}+1}$, we obtain a new feasible policy having a return only in the period $W_{i}$. The total cost of this latter will be modified by an amount of $e_{u}^{u, W_{i}+1}\left(\sum_{k=u}^{W_{i}-1} h_{k}-\right.$ $\left.p_{W_{i}}^{b}\right)$.

Second, we increase $e_{u}^{u, W_{i}+1}$ by $\epsilon$ with $\epsilon=\min \left\{q_{W_{i}}^{u, W_{i}+1}, \min _{k=u, \ldots, W_{i}-1} s_{k}\right\}<V$ and we decrease both the $s_{k}$ values $\left(k=u, \ldots, W_{i}-1\right)$ and $q_{W_{i}}^{u, W_{i}+1}$ by $\epsilon$. Hence, in this modified policy, we have either one subplan $\left(u, W_{i}+1\right)$ having a disposal, or a division of $\left(u, W_{i}+1\right)$ into at least two subplans knowing that in the first one we only have a disposal and in the last one there is only one return. Thus, the total cost will be modified by a quantity of $\epsilon\left(p_{W_{i}}^{b}-\sum_{k=u}^{W_{i}-1} h_{k}\right)$.

In the case where $p_{W_{i}}^{b}-\sum_{k=u}^{W_{i}-1} h_{k}=\sum_{k=u}^{W_{i}-1} h_{k}-p_{W_{i}}^{b}=0$, the solution remains optimal after the modifications of the current policy. In the case where $p_{W_{i}}^{b}-\sum_{k=u}^{W_{i}-1} h_{k} \neq 0$, the optimality is reached either by the first or the second modified policy which satisfies the property.

Define $\mathbb{1}_{\beta}$ as an indicator function, used in the next property:

$$
\mathbb{1}_{\beta}= \begin{cases}1 & \text { if } \beta \text { is true } \\ 0 & \text { otherwise }\end{cases}
$$

Property 3.4. Let $(u, v)$ be a subplan with $e_{u}^{u, v} \geq 0$ and let $j$ be a replenishment period in $(u, v)$. There exists an optimal policy such that, the ending inventory value of period $j-1$ is equal to $R_{u, j-1}-e_{u}^{u, v}+\mathbb{1}_{R_{u, j-1}<e_{u}^{u, v}} V, \forall j=u+1, \ldots, v$.

Proof. According to Property 3.1, there is an optimal solution such that the subplan $(u, v)$ has at most one disposal period placed at period $u$. In the case with $e_{u}^{u, v}>0$, if the retailer decides to order full batches in period $j$, then, the procurement of one batch at $j$ costs less than buying it in a previous period $t$ and storing it from $t$ to $j-1$. Thus, it is more expensive for the retailer to buy from period $u$ to $j-1$ a number of batches which satisfies both the demands of $u, \ldots, j-1$ with the disposed quantity $e_{u}^{u, v}$ and those after the periods $j$ (for example $\left\lceil\frac{D_{u, j}+e_{u}^{u, v}}{V}\right\rceil$ or $\left\lceil\frac{D_{u, j+1}+e_{u}^{u, v}}{V}\right\rceil$, etc.). Consequently, the quantity ordered in only full batches from period $u$ to period $j-1$ must be equal to $\left\lceil\frac{D_{u, j-1}+e_{u}^{u, v}}{V}\right\rceil$. Then, the number of batches replenished is either $\left\lceil\frac{D_{u, j-1}}{V}\right\rceil V$ or $\left(\left\lceil\frac{D_{u, j-1}}{V}\right\rceil+1\right) V$. Thus, what is left in period $j-1$ is either $R_{u, j-1}-e_{u}^{u, v}$ or $R_{u, j-1}-e_{u}^{u, v}+V$. It depends on the sign of $R_{u, j-1}-e_{u}^{u, v}$. In the case where $e_{u}^{u, v}=0$, it is easy to see that if $j$ is a replenishment period, then $s_{j-1}=R_{u, j-1}$.

Dynamic programming recursion to compute the optimal cost of $\llbracket 1, W_{i} \rrbracket$ :

To reduce the search space of the optimal solution of $\llbracket 1, W_{i} \rrbracket$, we use dominance properties in each subplan. Define $C_{v-1}$ as the optimal cost to satisfy the demand in periods $1,2, \ldots, v-1$, such that the entering inventory level of period $v$ is null $\left(s_{v-1}=0\right)$. Define $\mathscr{C}(u, v)$ as the optimal cost of each subplan $(u, v)$ satisfying the demand in periods $u, u+1, \ldots, v-1$, where 
$1 \leq u<v \leq W_{i}+1$. The following dynamic programming algorithm calculates the optimal total $\operatorname{cost} C_{W_{i}}$ and thus solves the subproblem $\llbracket 1, W_{i} \rrbracket$ :

Recurrence relation. For $v=2,3, \ldots, W_{i}+1$,

$$
C_{v-1}=\min _{1 \leq u<v}\left\{C_{u-1}+\mathscr{C}(u, v)\right\}
$$

Boundary condition. $C_{0}=0$

Objective. $C_{W_{i}}$

For the given subproblem $\llbracket 1, W_{i} \rrbracket$, the running time of the above dynamic programming algorithm is in $O\left(W_{i}^{2}\right)$ if all the $\mathscr{C}(u, v)$ values have been predetermined. We will show that finding all the $\mathscr{C}(u, v)$ values is bounded by time complexity $O\left(W_{i}^{4}\right)$. This implies that the subproblem $\llbracket 1, W_{i} \rrbracket$ is solvable in $O\left(W_{i}^{4}\right)$ time. The complexity of the general LSP-BD-R $\mathrm{R}_{1, \rho=1}$ is $O\left(\sum_{i=1}^{N}\left(W_{i}\right)^{4}\right)$ time. $f(x)=x^{4}$ is a super-additive function for non-negative real numbers, which means that we have $f(x)+f(y) \leq f(x+y)$. Therefore, we obtain $\sum_{i=1}^{N}\left(W_{i}\right)^{4} \leq\left(\sum_{i=1}^{N} W_{i}\right)^{4}=T^{4}$. Then, the general LSP-BD- $\mathrm{R}_{1, \rho=1}$ is solvable in $O\left(T^{4}\right)$ time. This efficiency can be improved through the use of Monge matrices proposed by Li et al. (2004) to be $O\left(W_{i}^{3} \log W_{i}\right)$ time for the subproblem $\llbracket 1, W_{i} \rrbracket$ and $O\left(T^{3} \log T\right)$ time for the whole problem.

Computation of $\mathscr{C}(u, v)$ :

To determine the cost $\mathscr{C}(u, v)$, we need to use Properties 3.1, 3.2, 3.3 and 3.4. In $(u, v)$ with $1 \leq u<v \leq W_{i}$, there exists an optimal solution such that there is at most one disposal and no return. The optimal cost of this subplan is denoted by $\mathscr{C}_{v}^{(1)}(u, v)$ explained below. However, in $\left(u, W_{i}+1\right)$ with $1 \leq u<W_{i}+1$, there is at most, either one disposal and no return with an optimal cost of $\mathscr{C}_{v}^{(1)}\left(u, W_{i}+1\right)$, or, one return and no disposal with an optimal cost denoted by $\mathscr{C}_{v}^{(2)}\left(u, W_{i}+1\right)$. In the above two cases, the idea of determining the cost of the subplan $(u, v)$ is to work on consecutive replenishment periods.

Let us define $\mathscr{C}_{v}^{(1)}(u, j+1)$ as the minimum cost to satisfy $d_{u}, d_{u+1}, \ldots, d_{j}$, such that in the subplan $(u, v)$ there is a disposal in period $u$ and period $j+1$ is a replenishment period with a known beginning inventory value (see Property 3.4), $1 \leq u \leq j \leq v-1 \leq W_{i}$. The dynamic programming formulation of $\mathscr{C}_{v}^{(1)}(u, j+1)$ is given as follows:

Recurrence relation. For $j=u, \ldots, v-1$,

$$
\begin{aligned}
\mathscr{C}_{v}^{(1)}(u, j+1)=\min _{u-1 \leq k<j}\{ & \mathscr{C}_{v}^{(1)}(u, k+1)+f_{k+1}+ \\
& \left(p_{k+1}+\frac{a_{k+1}}{V}\right)\left(\left(R_{u, j}-e_{u}^{u, v}+\mathbb{1}_{R_{u, j}<e_{u}^{u, v}} V\right)+D_{k+1, j}+\mathbb{1}_{k+1=u} e_{u}^{u, v}-\right. \\
& \left.\left.\mathbb{1}_{k+1>u}\left(R_{u, k}-e_{u}^{u, v}+\mathbb{1}_{R_{u, k}<e_{u}^{u, v}} V\right)\right)+H_{u, v}^{(1)}(k+1, j+1)\right\}
\end{aligned}
$$

Boundary condition. $\mathscr{C}_{v}^{(1)}(u, u)=0$

Objective. $\mathscr{C}_{v}^{(1)}(u, v)$

To get the $\mathscr{C}_{v}^{(1)}(u, j+1)$ value, we have to compute first all the minimum values of $\mathscr{C}_{v}^{(1)}(u, k+1)$ such that the periods $k+1$ and $j+1$ are two consecutive replenishment periods. Second, we compute the procurement cost at the beginning of the period $k+1$ by calculating the number of batches acquired for the periods $k+1, k+2, \ldots, j$ which is obtained by the known ending inventory 
levels of periods $k$ and $j$. And third, we compute the total holding cost $H_{u, v}^{(1)}(k+1, j+1)$ from the end of period $k+1$ up to the beginning of period $j+1$ knowing that $x_{k+2}=x_{k+3}=\cdots=x_{j}=0$, and $s_{j}=R_{u, j}-e_{u}^{u, v}+\mathbb{1}_{R_{u, j}<e_{u}^{u, v}} V$ for $j \leq v-1$.

All the values of $H_{u, v}^{(1)}(k+1, j+1)$ can be obtained recursively in $O\left(W_{i}^{4}\right)$ time as follows:

$$
\begin{cases}H_{u, v}^{(1)}(k+1, j+1)=\left(H_{u, v}^{(1)}(k+2, j+1)+\right. & \text { if } u-1 \leq k<j \leq v-1 \leq W_{i} \\ \left.h_{k+1}\left(D_{k+2, j}+\left(R_{u, j}-e_{u}^{u, v}+\mathbb{1}_{R_{u, j}<e_{u}^{u, v}} V\right)\right)\right) & \text { and } \mathbb{1}_{j \neq v-1 \text { or } k \neq v-2=1}=1 \leq W_{i} \\ H_{u, v}^{(1)}(j+1, j+1)=0 & \text { if } u \leq j \leq v-1 \leq W_{i}+1 \\ H_{u, v}^{(1)}(v-1, v)=0 & \text { if } 2 \leq v \leq W_{i}\end{cases}
$$

Define $\mathscr{C}_{v}^{(2)}(u, j+1)$ as the minimum cost to meet $d_{u}, d_{u+1}, \ldots, d_{j}$, such that in the subplan $(u, v)$ there is a return in period $W_{i}$ and period $j+1$ is a replenishment period with $1 \leq u \leq j \leq v-1=W_{i}$. The dynamic programming formulation of $\mathscr{C}_{v}^{(2)}(u, j+1)$ is given as follows:

Recurrence relation. For $j=u, \ldots, v-1$ and $v=W_{i}+1$,

$$
\begin{aligned}
\mathscr{C}_{v}^{(2)}(u, j+1)=\min _{u-1 \leq k<j}\{ & \mathscr{C}_{v}^{(2)}(u, k+1)+ \\
& f_{k+1}+\left(p_{k+1}+\frac{a_{k+1}}{V}\right)\left(R_{u, j}+D_{k+1, j}-R_{u, k}\right)+H_{u, v}^{(2)}(k+1, j+1)- \\
& \left.\mathbb{1}_{j=W_{i}} p_{W_{i}}^{b} R_{u, W_{i}}\right\}
\end{aligned}
$$

Boundary condition. $\mathscr{C}_{v}^{(2)}(u, u)=0$

Objective. $\mathscr{C}_{v}^{(2)}\left(u, W_{i}+1\right)$

To get the $\mathscr{C}_{v}^{(2)}(u, j+1)$ value, we follow the same procedure as calculating $\mathscr{C}_{v}^{(1)}(u, j+1)$ by modifying the disposal possibility by the return option, and thereafter, the ending inventory levels of periods $k$ and $j$ to $s_{k}^{u, v}=R_{u, k}, s_{j}^{u, v}=R_{u, j}$ for $j<W_{i}$, and for $j=W_{i}, s_{W_{i}}^{u, v}=0$ and $q_{W_{i}}^{u, W_{i}+1}=R_{u, W_{i}}$. All the values of $H_{u, v}^{(2)}(k+1, j+1)$ can be solved in $O\left(W_{i}^{4}\right)$ time knowing that they have the same equations of $H_{u, v}^{(1)}(k+1, j+1)$ with the only difference being $\left(R_{u, j}\right)$ instead of $\left(R_{u, j}-e_{u}^{u, v}+\mathbb{1}_{R_{u, j}<e_{u}^{u, v}} V\right)$.

Now, we can compute the possible values of $\mathscr{C}(u, v)$ in an $O\left(W_{i}^{4}\right)$ time according to the values of $(u, v)$ :

$$
\begin{cases}\mathscr{C}(u, v)=\mathscr{C}_{v}^{(1)}(u, v) & \text { if } 1 \leq u<v \leq W_{i} \\ \mathscr{C}\left(u, W_{i}+1\right)=\min \left\{\mathscr{C}_{v}^{(1)}\left(u, W_{i}+1\right), \mathscr{C}_{v}^{(2)}\left(u, W_{i}+1\right)\right\} & \text { if } 1 \leq u<v=W_{i}+1\end{cases}
$$

All the possible $\mathscr{C}(u, v)$ values are predetermined. Now, we can compute the optimal cost $C_{W_{i}}$ to satisfy the demands of the subproblem $\llbracket 1, W_{i} \rrbracket$ in only full batch with the disposal and return options in $O\left(W_{i}^{4}\right)$ time and the minimum total cost of LSP-BD-R $\mathrm{R}_{1, \rho=1}$ in $O\left(T^{4}\right)$ time.

LSP-BD-R $\mathbf{R}_{1, \rho=1}$ for the special case with $p_{W_{i}}^{b} \geq \sum_{k=1}^{W_{i}-1} h_{k}$

In this case, we suppose that the cumulative holding cost from period 1 to period $W_{i}-1$ is lower than the return revenue at period $W_{i}$. We show that the problem can be solved without decomposing it into subplans, which reduces its computational complexity. Hence, the key idea 
of this algorithm is to detect the ordering periods in order to compute the optimal total cost of $\llbracket 1, W_{i} \rrbracket$.

Property 3.5. For the subproblem $\llbracket 1, W_{i} \rrbracket$ with $p_{W_{i}}^{b} \geq \sum_{k=1}^{W_{i}-1} h_{k}$, there exists an optimal policy such that $e_{t}=0$ for each $t=1,2, \ldots, W_{i}$.

Proof. We consider a policy $\pi^{\prime}$ in which we have $e_{t}>0$ in $\llbracket 1, W_{i} \rrbracket$. We follow the same procedure of the first part of the proof of Property 3.3 by modifying $e_{u}^{u, W_{i}+1}$ by $e_{t}$. The policy $\pi^{\prime}$ cannot be optimal because of the hypothesis $p_{W_{i}}^{b}-\sum_{k=t}^{W_{i}-1} h_{k} \geq 0, \forall t \in\left\{1, \ldots, W_{i}\right\}$.

This property implies that there is no discarded quantities at the retailer level over $\llbracket 1, W_{i} \rrbracket$, thus, all the left-over items are sold to the supplier at the end of period $W_{i}$. This special problem is already studied in Chapter 2 (see Subsections 2.3.1 and 2.3.2). We have proposed an $O\left(W_{i}^{2}\right)$ time dynamic programming algorithm for the given interval $1, \ldots, W_{i}$. If all the subproblems have their holding cost structure such that $\sum_{k=1}^{W_{i}-1} h_{k} \leq p_{W_{i}}^{b}$ for all $i \in\{1, \ldots, N\}$, then, LSPBD- $\mathrm{R}_{1, \rho=1}$ can be solved in $O\left(T^{2}\right)$ time.

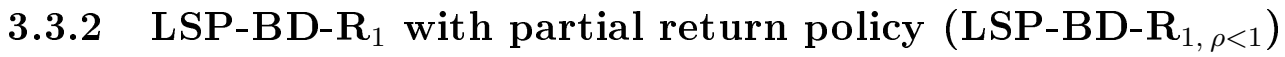

In this problem, the returned quantity in period $W_{i}$ is limited by $\rho \sum_{k=1}^{W_{i}} x_{k}$ with $\rho<1$. As previously, we solve each subproblem $\llbracket w_{i-1}+1, w_{i} \rrbracket$ for all $i=1, \ldots, N$ as $\llbracket 1, W_{i} \rrbracket$. Properties and algorithms are established for the general case with linear holding costs and for the special case with $p_{W_{i}}^{b} \geq \sum_{k=1}^{W_{i}-1} h_{k}$.

\section{LSP-BD-R $\mathbf{R}_{1, \rho<1}$ for the general case}

The only constraint changing between LSP-BD-R $\mathrm{R}_{1, \rho<1}$ and LSP-BD-R $\mathrm{R}_{1, \rho=1}$ is: $q_{W_{i}} \leq \rho \sum_{k=1}^{W_{i}} x_{k}$ with $\rho$ being less than 1 . The idea of the algorithm is the same as that of LSP-BD- $R_{1, \rho=1}$, but we add the fact that the retailer can increase his purchases to return as many products as possible. We present two examples for LSP-BD-R $\mathrm{R}_{1}$ in order to show the difference between the optimal plans of the two cases: $\rho=1$ and $\rho<1$.

Example 3.1. Consider a time horizon of 3 periods in which the demands $(1,1,1)$ have to be satisfied in full batches of size $V=2$ units. Unit procurement and holding costs are set to 3 and 6 per product and per unit of time, respectively. A fixed cost per batch of 1 is paid per period. Setup costs are null. The unsold items can be returned to the supplier only at the end of period 3 for a revenue of 2 .

Table 3.1 shows the optimal plans of LSP-BD-R ${ }_{1, \rho=1}$ and LSP-BD-R ${ }_{1, \rho=\frac{1}{6}}$ indicating that there is one independent subproblem $\llbracket 1,3 \rrbracket$ to optimize. It is interesting to note that, in the case where $\rho=\frac{1}{6}$, the retailer purchases one additional batch at period 2 compared to the case where $\rho=1$, in which $\left\lceil\frac{d_{1}+d_{2}}{V}\right\rceil$ batches are replenished for periods 1 and 2 . The purchased quantities are thus increased to return the maximum possible value for $\rho<1$.

Example 3.2. We consider the same data given in Example 3.1 by changing the value of the batch size by 4 units and we choose $\rho=\frac{1}{9}$ in order to see the difference between the cases $\rho=1$ and $\rho<1$.

We note that in the case where we have $\rho=\frac{1}{9}$, the retailer purchases one less batch at period 3 compared to the case where we have $\rho=1$ in which $\left\lceil\frac{d_{1}+d_{2}+d_{3}}{V}\right\rceil+V$ batches are purchased for 
Table 3.1: The optimal quantities of LSP-BD-R ${ }_{1, \rho=1}$ and LSP-BD-R ${ }_{1, \rho=\frac{1}{6}}$ for $V=2$

\begin{tabular}{c|c|cccc||cccc}
\multicolumn{1}{l}{} & \multicolumn{4}{c}{ LSP-BD-R $_{1, \rho=1}$} & \multicolumn{4}{c}{ LSP-BD-R $_{1, \rho=\frac{1}{6}}$} \\
\hline Period $t$ & $d_{t}$ & $x_{t}$ & $s_{t}$ & $q_{t}$ & $e_{t}$ & $x_{t}$ & $s_{t}$ & $q_{t}$ & $e_{t}$ \\
\hline 1 & 1 & 2 & 1 & 0 & 0 & 2 & 0 & 0 & 1 \\
2 & 1 & 0 & 0 & 0 & 0 & 2 & 0 & 0 & 1 \\
3 & 1 & 2 & 0 & 1 & 0 & 2 & 0 & 1 & 0 \\
\hline
\end{tabular}

Table 3.2: The optimal quantities of LSP-BD-R ${ }_{1, \rho=1}$ and LSP-BD-R $\mathrm{R}_{1, \rho=\frac{1}{9}}$ for $V=4$

\begin{tabular}{c|c|cccc||cccc}
\multicolumn{1}{l}{} & \multicolumn{4}{c}{ LSP-BD-R ${ }_{1, \rho=1}$} & \multicolumn{4}{c}{ LSP-BD-R $_{1, \rho=\frac{1}{9}}$} \\
\hline Period $t$ & $d_{t}$ & $x_{t}$ & $s_{t}$ & $q_{t}$ & $e_{t}$ & $x_{t}$ & $s_{t}$ & $q_{t}$ & $e_{t}$ \\
\hline 1 & 1 & 4 & 1 & 0 & 2 & 4 & 2 & 0 & 1 \\
2 & 1 & 0 & 0 & 0 & 0 & 0 & 1 & 0 & 0 \\
3 & 1 & 4 & 0 & 3 & 0 & 0 & 0 & 0 & 0 \\
\hline
\end{tabular}

periods 1,2 and 3 .

We observe that the purchased quantities throughout a subproblem in the case where $\rho<1$ can be more or less than the ones in the case where we have $\rho=1$. However, they must be equal to or greater than the number of batches satisfying all the demands $\left(\left\lceil\frac{d_{1}+\cdots+d_{W_{i}}}{V}\right\rceil V\right)$.

Some preliminaries and properties:

Properties 3.1 and 3.2 introduced in the previous section for the case with full return remain valid for LSP-BD-R $1, \rho<1$. Recall that, for any optimal subplan $(u, v)$ such that $1 \leq u<v \leq W_{i}$, there is at most one disposal period placed in period $u$ without return and we can easily compute its quantity.

Property 3.6. For the subplan $(u, v)$ such that $1 \leq u<v=W_{i}+1$, there exists, in an optimal planning strategy, at most either one disposal in period $u$ if returning is less profitable than disposing, or one disposal in $u$ and one return in period $W_{i}$ at the same time. Therefore, in the second possibility, we have $e_{u}^{u, W_{i}+1}+q_{W_{i}}^{u, W_{i}+1}=\left\lceil\frac{D_{u, W_{i}}}{V}\right\rceil V-D_{u, W_{i}}$, then we can have either one disposal, or one return, or both, or nothing.

Property 3.6 is a modification of Property 3.3, because in this problem the returned quantity depends on the buyback percentage $\rho$. The fact that there is at most one disposal period is proved by Property 3.1. Besides, in the subplan $\left(u, W_{i}+1\right)$, there is at most one return period being $W_{i}$.

In the calculation of the optimal cost of a subplan with only one disposal, we use Property 3.4 in which in any optimal solution, the starting inventory level of a replenishment period $j$ for $2 \leq j \leq W_{i}+1$ is $R_{u, j-1}-e_{u}^{u, v}+\mathbb{1}_{R_{u, j-1}<e_{u}^{u, v}} V$ by replacing the value of $e_{u}^{u, v}$ according to each subplan. For a subplan with one disposal and one return, we also apply Property 3.4 for $2 \leq j \leq W_{i}$, and, for $j=W_{i}+1$ we have $s_{W_{i}}=0$.

Property 3.7. The optimal number of batches replenished from period 1 to period $k$ with $s_{k}=0$ is equal to $\left\lceil\frac{D_{1, k}}{V}\right\rceil+\Delta$, with $\Delta$ taking its value from the set $\{0, \ldots, k-1\}, \forall k=1, \ldots, W_{i}$. 
Proof. It is easy to see that the minimum number of batches to replenish is $\left\lceil\frac{D_{1, k}}{V}\right\rceil$ in order to only satisfy the total demand $d_{1}+\cdots+d_{k}$ to be feasible. The maximum number of batches to replenish is $\sum_{l=1}^{k}\left\lceil\frac{d_{l}}{V}\right\rceil$ because the maximum number of subplans is $k:(1,2),(2,3), \ldots,(k, k+1)$. In each of those $k$ subplans, we have to meet the demands. In each period $l$, the retailer orders batches to satisfy only $d_{l}$ and disposes and/or returns what is left. It is easy to see that the value $\sum_{l=1}^{k}\left\lceil\frac{d_{l}}{V}\right\rceil$ can be at most $\left\lceil\frac{D_{1, k}}{V}\right\rceil+(k-1)$ (based on the fact that for $\left.a, b \in \mathbb{R}_{+},\lceil a\rceil+\lceil b\rceil \leq\lceil a+b\rceil+1\right)$.

Dynamic programming recursion to compute the optimal cost of $\llbracket 1, W_{i} \rrbracket$ :

The idea is first to decompose the subproblem $\llbracket 1, W_{i} \rrbracket$ as before into subplans $(u, v)$. Then, we compute all the optimal $\operatorname{costs} C^{\rho}(v, \Delta)$ for $v=2,3, \ldots, W_{i}+1$ and $\Delta=0, \ldots, v-2$ which corresponds to the total cost to satisfy the demand $D_{1, v-1}$ knowing that we have $s_{v-1}=0$ and $\Delta$ is the number of additional batch replenished from period 1 to period $v-1$. Once this cost is computed for all the values of $v$, we obtain the costs $C^{\rho}\left(W_{i}+1, \Delta\right)$ for all possible values of $\Delta$. At the end, we compute the optimal total cost of $\llbracket 1, W_{i} \rrbracket$ which is the minimum of $C^{\rho}\left(W_{i}+1, \Delta\right)$, among the $\Delta$ values.

To obtain these costs, the time horizon $1, \ldots, v-1$ with a total number of batches to buy being $\left(\left\lceil\frac{D_{1, v-1}}{V}\right\rceil+\Delta\right)$ is divided into the time horizon $1, \ldots, u-1$ with $s_{u-1}=0$ and the subplan $(u, v)$, (see Figure 3.2). Throughout the time horizon $1, \ldots, u-1$, we know the number of batches purchased, which is $\left\lceil\frac{D_{1, u-1}}{V}\right\rceil+\Delta^{\prime}$ with $\Delta^{\prime}$ being the extra batches replenished from period 1 to period $u-1$. Besides, in the subplan $(u, v)$, the number of batches purchased from period $u$ till $v-1$ is equal to $\left\lceil\frac{D_{u, v-1}}{V}\right\rceil$. Hence, we can easily compute the value of $\Delta^{\prime}$ : $\Delta^{\prime}=\Delta+\left\lceil\frac{D_{1, v-1}}{V}\right\rceil-\left\lceil\frac{D_{1, u-1}}{V}\right\rceil-\left\lceil\frac{D_{u, v-1}}{V}\right\rceil$. For a given value of $\Delta$, the value of $\Delta^{\prime}$ is thus known.

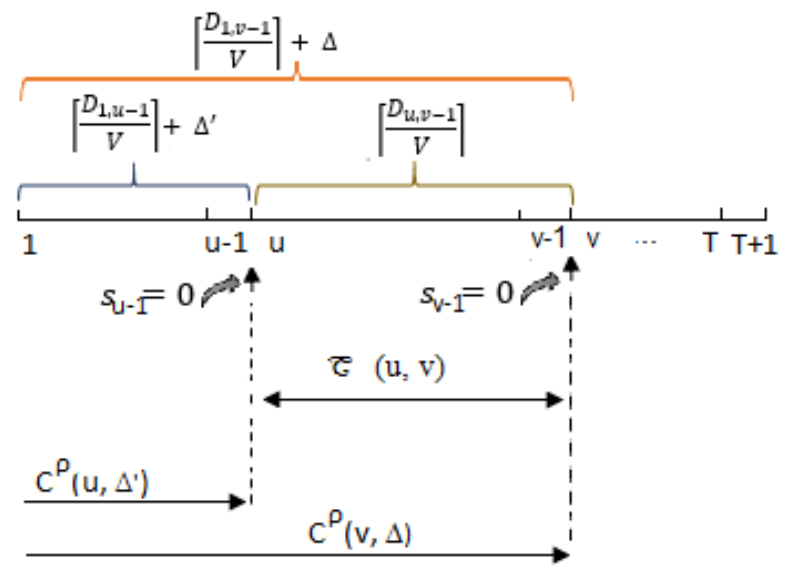

Figure 3.2: How to compute the optimal costs $C^{\rho}(v, \Delta)$

The costs $C^{\rho}(v, \Delta)$ are computed by the following algorithm:

Recurrence relation. For $v=2,3, \ldots, W_{i}+1$ and $\Delta=0, \ldots, v-2$,

$$
C^{\rho}(v, \Delta)=\min _{1 \leq u<v}\left\{C^{\rho}\left(u, \Delta^{\prime}\right)+\mathscr{C}(u, v)\right\}
$$

Boundary condition. $C^{\rho}(1,0)=0, C^{\rho}(v, \Delta)=+\infty$ with $\Delta \geq v-1$, or $\Delta<0$ 
Objective. $C^{\rho}\left(W_{i}+1, \Delta\right)$

The time complexity of this shortest path algorithm is $O\left(W_{i}^{4}\right)$ knowing that all the optimal subplan costs $\mathscr{C}(u, v)$ will be computed in time complexity $O\left(W_{i}^{4}\right)$. The total optimal cost of the subproblem $\llbracket 1, W_{i} \rrbracket$ with $\rho<1$ is denoted by $\operatorname{Cost}_{\min }\left(W_{i}+1\right)$ and is computed in $O\left(W_{i}^{5}\right)$ time as follows:

$$
\operatorname{Cost}_{\text {min }}\left(W_{i}+1\right)=\min _{0 \leq \Delta \leq W_{i}-1}\left\{C^{\rho}\left(W_{i}+1, \Delta\right)\right\}
$$

Therefore, the complexity of the whole LSP-BD- $\mathrm{R}_{1, \rho<1}$ in the general case is solvable in $O\left(T^{5}\right)$ time.

\section{Computation of $\mathscr{C}(u, v)$ :}

We have to calculate the optimal cost of all subplans. There are two possibilities in a given suplan $(u, v)$ : we have either a subplan with at most one disposal period, or, a subplan with at most one disposal period and one return period (the disposal and return quantities will be subsequently calculated in terms of $\rho$, in order to find out if there is a disposal, or a return, or both, or nothing). Thus, there are two possible values of $\mathscr{C}(u, v)$.

For $1 \leq u<v \leq W_{i}$, the optimal cost is computed as previously $\mathscr{C}(u, v)=\mathscr{C}_{v}^{(1)}(u, v)$.

For $1 \leq u<v=W_{i}+1$, the optimal cost of the last subplan $\left(u, W_{i}+1\right)$ is computed as follows:

$$
\left.\mathscr{C}\left(u, W_{i}+1\right)=\min \left\{\mathscr{C}_{W_{i}+1}^{(1)}\left(u, W_{i}+1\right), \mathscr{C}^{\Delta}\left(u, W_{i}+1\right)\right\}\right\}
$$

The first possible cost $\mathscr{C}_{v}^{(1)}(u, v)$ is addressed in the previous section and $\mathscr{C}^{\Delta}\left(u, W_{i}+1\right)$ is the minimum cost of the subplan $\left(u, W_{i}+1\right)$ having at most a disposal and a return such that $W_{i}+1$ is a replenishment period and $\Delta$ is the total additional batches replenished from period 1 to period $W_{i}$. To determine $\mathscr{C}^{\Delta}\left(u, W_{i}+1\right)$, we have to compute the returned quantity which depends on $\Delta$ (denoted by $\left.q_{W_{i}}^{u, W_{i}+1}(\Delta)\right)$ and the disposed quantity denoted by $e_{u}^{u, W_{i}+1}(\Delta)$. We know that the quantity purchased throughout the overall planning horizon is equal to $\left\lceil\frac{D_{1, W_{i}}}{V}\right\rceil V+\Delta V$. Hence, we obtain $q_{W_{i}}^{u, W_{i}+1}(\Delta) \leq B S\left(u, W_{i}, \Delta\right)$ with $B S\left(u, W_{i}, \Delta\right)$ corresponding to the upper limit of $q_{W_{i}}^{u, W_{i}+1}(\Delta), B S\left(u, W_{i}, \Delta\right)=\left\lfloor\rho\left(\left\lceil\frac{D_{1, W_{i}}}{V}\right\rceil V+\Delta V\right)\right\rfloor$. For each $u$ such that $u=1, \ldots, W_{i}$, in the subplan $\left(u, W_{i}+1\right)$, we compare between the remaining quantity $\left\lceil\frac{D_{u, W_{i}}}{V}\right\rceil V-D_{u, W_{i}}$ and the one of $B S\left(u, W_{i}, \Delta\right)$ :

- If $\left\lceil\frac{D_{u, W_{i}}}{V}\right\rceil V-D_{u, W_{i}} \leq B S\left(u, W_{i}, \Delta\right)$, then the retailer returns all the remaining quantity of $\left(u, W_{i}+1\right), q_{W_{i}}^{u, W_{i}+1}(\Delta)=\left\lceil\frac{D_{u, W_{i}}}{V}\right\rceil V-D_{u, W_{i}}$, and makes no disposal, i.e., $e_{u}^{u, W_{i}+1}(\Delta)=0$. In this case, the optimal cost of the subplan $\left(u, W_{i}+1\right)$ is equal to $\mathscr{C}_{W_{i}+1}^{(2)}\left(u, W_{i}+1\right)$.

- Otherwise, the retailer only returns the maximum amount, that can be returned, depending on the value of $\rho, q_{W_{i}}^{u, W_{i}+1}(\Delta)=B S\left(u, W_{i}, \Delta\right)$, and gets rid of what is left after returning, $e_{u}^{u, W_{i}+1}(\Delta)=\left\lceil\frac{D_{u, W_{i}}}{V}\right\rceil V-D_{u, W_{i}}-B S\left(u, W_{i}, \Delta\right)$. The dynamic programming formulation used to obtain $\mathscr{C}^{\Delta}\left(u, W_{i}+1\right)$ is the same as that of $\mathscr{C}_{W_{i}+1}^{(1)}\left(u, W_{i}+1\right)$, with the additional consideration of the return revenue at period $W_{i}$. 


\section{LSP-BD-R $\mathbf{R}_{1, \rho<1}$ for the special case with $p_{W_{i}}^{b} \geq \sum_{k=1}^{W_{i}-1} h_{k}$}

To solve the special case of LSP-BD- $\mathrm{R}_{1, \rho<1}$ with $p_{W_{i}}^{b} \geq \sum_{k=1}^{W_{i}-1} h_{k}$, we keep the same properties as LSP-BD-R $1, \rho<1$, except Property 3.7. Instead, we propose Property 3.8.

Property 3.8. In an optimal plan, the retailer acquires a minimum number of batches to satisfy the demand from period 1 to period $w$. Hence, the number of optimal batches replenished in this duration is equal to $\left\lceil\frac{D_{1, w}}{V}\right\rceil$, which means that, we always have $\Delta=0$.

Proof. We suppose that there is an optimal policy $\pi^{\prime}$ in which the retailer orders the quantity $\left\lceil\frac{D_{1, w}}{V}\right\rceil V+V$ for the periods $1, \ldots, w$. We can consider another policy $\pi^{*}$ which only orders $\left\lceil\frac{D_{1, w}}{V}\right\rceil$. Since $\pi^{\prime}$ is optimal, the total cost of $\pi^{\prime}$ is less than the one of $\pi^{*}\left(\mathscr{C}\left(\pi^{\prime}\right) \leq \mathscr{C}\left(\pi^{*}\right)\right)$.

In order to obtain the policy $\pi^{*}$, we have to modify the policy $\pi^{\prime}$ and calculate the difference between $\mathscr{C}\left(\pi^{\prime}\right)$ and $\mathscr{C}\left(\pi^{*}\right)$. We choose all the periods of $\pi^{\prime}$ in the planning horizon with $e_{t}^{\prime}>0$, let us take two periods: $t_{1}, t_{2}$ with $t_{1}<t_{2}$. We will reduce the quantity $\sum_{k=1}^{W_{i}} x_{k}^{\prime}=\left\lceil\frac{D_{1, W_{i}}}{V}\right\rceil V+V$ purchased in policy $\pi^{\prime}$ by a batch size $V$ by decreasing the disposed and returned quantities by $V$. Indeed, let $V=\alpha_{1}+\alpha_{2}+\beta$ such that $\alpha_{1} \leq e_{t_{1}}^{\prime}, \alpha_{2} \leq e_{t_{2}}^{\prime}, q_{W_{i}}^{\prime}-\rho\left\lceil\frac{D_{1, W_{i}}}{V}\right\rceil V \leq \beta \leq q_{W_{i}}^{\prime}$ and $\alpha_{1}, \alpha_{2}, \beta \in \mathbb{N}$.

We only modify the following variables by leaving the others unchanged.

$-e_{t_{1}}^{*}=e_{t_{1}}^{\prime}-\alpha_{1}$,

- $s_{c}^{*}=s_{c}^{\prime}+\alpha_{1}, \forall c \in\left\{t_{1}, \ldots, t_{2}-1\right\}$,

$-e_{t_{2}}^{*}=e_{t_{2}}^{\prime}-\alpha_{2}$

- $s_{c}^{*}=s_{c}^{\prime}+\alpha_{1}+\alpha_{2}, \forall c \in\left\{t_{2}, \ldots, t_{3}-1\right\}$, with $t_{3}$ being the last ordering period in $\pi^{\prime}$.

$-x_{t_{3}}^{*}=x_{t_{3}}^{\prime}-V$,

- $s_{c}^{*}=s_{c}^{\prime}-\beta, \forall c \in\left\{t_{3}, \ldots, W_{i}-1\right\}$, we have absolutely $\beta \leq q_{W_{i}}^{\prime} \leq \min _{c=t_{3}, \ldots, W_{i}-1} s_{c}^{\prime}$

- $q_{W_{i}}^{*}=q_{W_{i}}^{\prime}-\beta$.

We obtain the following relation between the costs $\mathscr{C}\left(\pi^{*}\right)$ and $\mathscr{C}\left(\pi^{\prime}\right)$ :

$$
\mathscr{C}\left(\pi^{*}\right)=\mathscr{C}\left(\pi^{\prime}\right)-\left(V\left(p_{t_{3}}+\frac{a_{t_{3}}}{V}\right)-\alpha_{1} \sum_{c=t_{1}}^{t_{2}-1} h_{c}-\left(\alpha_{1}+\alpha_{2}\right) \sum_{c=t_{2}}^{t_{3}-1} h_{c}-\beta p_{W_{i}}^{b}\right)-\beta \sum_{c=t_{3}}^{W_{i}-1} h_{c}
$$

Since the gross marginal procurement cost after returning the unused products $\left(p_{W_{i}}^{b}<p_{t_{3}}\right)$ is positive for each period, then, we obtain $(V-\beta) p_{t_{3}}<V p_{t_{3}}-\beta p_{W_{i}}^{b}$. Under the latter buyback contract assumption and the special case of this problem $\left(p_{W_{i}}^{b} \geq \sum_{k=1}^{W_{i}-1} h_{k}\right)$, we get $p_{t_{3}}>\sum_{c=t_{1}}^{t_{2}-1} h_{c}+\sum_{c=t_{2}}^{t_{3}-1} h_{c}$. Hence, we obtain $(V-\beta) p_{t_{3}}>\alpha_{1} \sum_{c=t_{1}}^{t_{2}-1} h_{c}+\left(\alpha_{1}+\alpha_{2}\right) \sum_{c=t_{2}}^{t_{3}-1} h_{c}$, because we have $V-\beta \geq \alpha_{1}$ and $V-\beta=\alpha_{1}+\alpha_{2}$. Therefore, we have $0<(V-\beta)\left(p_{t_{3}}+\frac{a_{3}}{V}\right)-$ $\alpha_{1} \sum_{c=t_{1}}^{t_{2}-1} h_{c}-\left(\alpha_{1}+\alpha_{2}\right) \sum_{c=t_{2}}^{t_{3}-1} h_{c}<V\left(p_{t_{3}}+\frac{a_{t_{3}}}{V}\right)-\alpha_{1} \sum_{c=t_{1}}^{t_{2}-1} h_{c}-\left(\alpha_{1}+\alpha_{2}\right) \sum_{c=t_{2}}^{t_{3}-1} h_{c}-\beta p_{W_{i}}^{b}$. It means that $\mathscr{C}\left(\pi^{\prime}\right)>\mathscr{C}\left(\pi^{*}\right)$, which contradicts the optimality of $\pi^{\prime}$.

According to Property 3.8, we do not take into account the extra number of batches $\Delta$ to replenish from period 1 to period $k$ with $s_{k}=0$. Thus, the complexity of the subproblem $\llbracket 1, W_{i} \rrbracket$ with $p_{W_{i}}^{b} \geq \sum_{k=1}^{W_{i}-1} h_{k}$ is reduced to $O\left(W_{i}^{4}\right)$ time. Thereafter, the running time of the overall problem reduces to $O\left(T^{4}\right)$ time. 
We note that the algorithm proposed for the LSP-BD-R ${ }_{1, \rho<1}$ can solve the LSP-BD- $\mathrm{R}_{1, \rho=1}$ but with a higher complexity.

\subsection{LSP-BD-R with time limit for returns (LSP-BD-R $\left.{ }_{2}\right)$}

In this section, return periods become decision variables. The supplier also imposes some restrictions on the returns. These include on the one hand the strict time limit on returns, and, on the other hand the returned quantity limit. This type of contract presents the way of controlling excessive return to the supplier. Such a policy is common in the industries of home-electronics, computers, etc. The return periods and the total number of return over the planning horizon are unknown (decision variables) and they will be optimized in the model.

Over the first $j$ periods (with $j$ a given period fixed in the contract, $j \leq T$ ), the supplier allows returns any time until $j$ (with $\mathrm{j}$ included). After the period $j$, the returns are forbidden. The total quantity to return over $1 . . j$ is limited to $\rho \sum_{i=1}^{j} x_{i}$ units $\left(\sum_{i=1}^{j} q_{i} \leq \rho \sum_{i=1}^{j} x_{i}\right)$. For one unit returned in period $i$ (with $i \leq j$ ), we have a return revenue $p_{i}^{b}$, and we assume $\max _{i=1, \ldots, j}\left\{p_{i}^{b}\right\}<$ $p_{t}, \forall t \in\{1, \ldots, j\}$ in order to avoid the speculation.

Below, we give an MILP formulation without inventory variables whose objective is to minimize all the costs over the planning horizon. Note that such a transformation is possible for the case of linear holding cost. In this section, we need to transform the aggregate formulation of this problem into the following one in order to facilitate the understanding of some dominance properties. The proof of the equivalence between the two formulations is explained in Appendix. Let $\tilde{p}_{t}$ be the modified unit procurement cost in period $t$ coupled with cumulative holding costs of periods $t, \ldots, T, \tilde{p_{t}}=p_{t}+\sum_{k=t}^{T} h_{k}$, let $\tilde{p_{t}^{b}}$ be the modified return revenue in period $t$ after integrating the cumulative holding cost, $\tilde{p}_{t}^{b}=p_{t}^{b}+\sum_{k=t}^{T} h_{k}$, and let $\tilde{p_{t}^{e}}$ be the modified disposal revenue or cost taking into account the inventory holding costs from period $t$ to period $T$, $\tilde{p}_{t}^{e}=p_{t}^{e}+\sum_{k=t}^{T} h_{k}$ with $p_{t}^{e}=0$.

$$
\min \sum_{t=1}^{T}\left(f_{t} y_{t}+\tilde{p_{t}} x_{t}+a_{t} A_{t}-\tilde{p_{t}^{b}} q_{t}-\tilde{p_{t}^{e}} e_{t}-h_{t} \sum_{i=1}^{t} d_{i}\right)
$$

Constraints without inventory variables:

$$
\sum_{k=1}^{t} x_{k} \geq \sum_{k=1}^{t}\left(d_{k}+q_{k}+e_{k}\right) \quad \forall t=1, \ldots, T
$$

Conditions of the buyback contract type $\mathrm{R}_{2}$ :

$$
\begin{aligned}
& \sum_{i=1}^{j} q_{i} \leq \rho \sum_{i=1}^{j} x_{i} \\
& \sum_{i=j+1}^{T} q_{i}=0
\end{aligned}
$$

Setup variable generation constraints:

$$
x_{t} \leq\left\lceil\frac{\sum_{j=t}^{T} d_{j}}{V_{t}}\right\rceil V y_{t}
$$$$
\forall t=1, \ldots, T
$$

Only full batch replenishment constraints: $x_{t}=A_{t} V$ $\forall t=1, \ldots, T$

Nonnegativity and binary constraints:

$$
x_{t}, q_{t}, e_{t} \in \mathbb{R}_{+}
$$$$
\forall t=1, \ldots, T
$$$$
A_{t} \in \mathbb{N}, y_{t} \in\{0,1\}
$$

$\forall t=1, \ldots, T$ 
We note that the last expression in the objective function is constant and thus, can be omitted. We study LSP-BD-R $R_{2}$ in the case of full and partial returns $(\rho=1$ and $\rho<1)$, respectively.

\subsubsection{LSP-BD-R $\mathbf{R}_{2}$ with full return policy (LSP-BD-R $\mathbf{R}_{2, \rho=1}$ )}

In this section, the total returned quantity over the first $j$ periods is limited by the total ordered quantity over the same periods. To solve our LSP-BD-R $2, \rho=1$, we proceed to decompose this problem into subplans $(u, v)$ as in Section 3.3. Note that, here we tackle one problem over the entire horizon 1..T. We will compute the minimum cost $\mathscr{C}(u, v)$ of each subplan $(u, v)$ with $1 \leq u<v \leq T+1$ according to the properties proposed in the following for LSP-BD- $\mathrm{R}_{2, \rho=1}$. By using Equation (3.1), we obtain the total optimal cost $C_{T}$ to satisfy the demands $d_{1}, d_{2}, \ldots, d_{T}$ in which we decide how much to order, to return and to dispose of over periods $1, \ldots, T$. We present an example to show the results of LSP-BD- $R_{2, \rho=1}$ for different values of $j$ and compare them with the ones of LSP-BD-R ${ }_{1, \rho=1}$.

Example 3.3. We use the same data given in Example 3.2 to compare the results of LSP-BD$\mathrm{R}_{2, \rho=1}$ for different return limits $j \in\{1,2,3\}$, with those of LSP-BD- $\mathrm{R}_{1, \rho=1}$ (see Table 3.3). We consider $p_{1}^{b}=p_{2}^{b}=1, p_{3}^{b}=2$. Notice that for LSP-BD-R L $, \rho=1_{1}, \forall j \in\{1,2,3\}$, there is at most one return in each subplan in which the buyback is allowed. We note that, if we increase the value of $j$, then the cost of LSP-BD- $\mathrm{R}_{2, \rho=1}$ decreases. Besides, we note that the cost of LSP-BD- $\mathrm{R}_{2, \rho=1}$ with $j=3$ is less than the one of LSP-BD-R ${ }_{1, \rho=1}$. In LSP-BD- $\mathrm{R}_{2, \rho=1}$ with $j=T=3$, there are no restrictions on the return throughout the planning horizon, but in LSP-BD- $\mathrm{R}_{1, \rho=1}$, the returns are forced to be in specific periods.

Table 3.3: The optimal quantities of LSP-BD-R ${ }_{2, \rho=1}$ for $j=1,2,3$ and LSP-BD-R ${ }_{1, \rho=1}: V=4$

\begin{tabular}{|c|c|c|c|c|c|c|c|c|c|c|c|c|c|c|c|c|}
\hline$V=4$ & \multicolumn{4}{|c|}{ LSP-BD-R $2, \rho=1 j=1$} & \multicolumn{4}{|c|}{ LSP-BD-R $2, \rho=1 j=2$} & \multicolumn{4}{|c|}{ LSP-BD-R $_{2, \rho=1} j=3$} & \multicolumn{4}{|c|}{ LSP-BD-R $1, \rho=1$} \\
\hline Period $t$ & $x_{t}$ & $s_{t}$ & $q_{t}$ & $e_{t}$ & $x_{t}$ & $s_{t}$ & $q_{t}$ & $e_{t}$ & $x_{t}$ & $s_{t}$ & $q_{t}$ & $e_{t}$ & $x_{t}$ & $s_{t}$ & $q_{t}$ & $e_{t}$ \\
\hline 1 & 4 & 0 & 3 & 0 & 4 & 0 & 3 & 0 & 4 & 1 & 2 & 0 & 4 & 1 & 0 & 2 \\
\hline 2 & 4 & 1 & 0 & 2 & 4 & 1 & 2 & 0 & 0 & 0 & 0 & 0 & 0 & 0 & 0 & 0 \\
\hline 3 & 0 & 0 & 0 & 0 & 0 & 0 & 0 & 0 & 4 & 0 & 3 & 0 & 4 & 0 & 3 & 0 \\
\hline Costs & \multicolumn{4}{|c|}{29} & \multicolumn{4}{|c|}{27} & \multicolumn{4}{|c|}{24} & \multicolumn{4}{|c|}{26} \\
\hline
\end{tabular}

In what follows, we give a polynomial time algorithm running in $O\left(T^{5}\right)$ time for LSP-BD$\mathrm{R}_{2, \rho=1}$. We first present several optimality properties.

Some preliminaries and properties:

Property 3.9. If there exists an optimal schedule in which the periods $t_{1}, t_{2}, \ldots, t_{n}$ are $n$ ordering periods such that $t_{1}<t_{2}<\cdots<t_{n}$, then, the total unit costs at these periods verify $\tilde{p}_{t_{1}}+\frac{a_{t_{1}}}{V} \geq \tilde{p}_{t_{2}}+\frac{a_{t_{2}}}{V} \geq \cdots \geq \tilde{p}_{t_{n}}+\frac{a_{t_{n}}}{V}$.

This property is used in the proof of Property 3.11.

Proof. We will show that in a policy with two periods $t_{1}$ and $t_{2}$ such that $t_{1}<t_{2}$ and $\tilde{p}_{t_{1}}+\frac{a_{t_{1}}}{V}<$ $\tilde{p}_{t_{2}}+\frac{a t_{2}}{V}$, periods $t_{1}$ and $t_{2}$ cannot be both ordering periods.

We consider an optimal policy $\pi^{\prime}$ in which $t_{1}$ and $t_{2}$ are two ordering periods $\left(x_{t_{1}}^{\prime}>0\right.$ and $\left.x_{t_{2}}^{\prime}>0\right)$ with $\tilde{p}_{t_{1}}+\frac{a_{t_{1}}}{V}<\tilde{p}_{t_{2}}+\frac{a_{t_{2}}}{V}$. We modify the policy $\pi^{\prime}$ to obtain a policy $\pi^{*}$ with a feasible solution as follows: $x_{t_{1}}^{*}=x_{t_{1}}^{\prime}+x_{t_{2}}^{\prime}, s_{k}^{*}=s_{k}^{\prime}+x_{t_{2}}^{\prime}, \forall k \in\left\{t_{1}, \ldots, t_{2}-1\right\}$ and $x_{t_{2}}^{*}=0$. Now, we compare the costs of these two policies: $\mathscr{C}\left(\pi^{*}\right)=\mathscr{C}\left(\pi^{\prime}\right)-x_{t_{2}}^{\prime}\left(\left(\tilde{p}_{t_{2}}+\frac{a_{t_{2}}}{V}\right)-\left(\tilde{p}_{t_{1}}+\frac{a_{t_{1}}}{V}\right)\right)-f_{t_{2}}$. The 
change of policy $\pi^{\prime}$ decreases the cost of policy $\pi^{*}$ in which period $t_{1}$ is an ordering period but period $t_{2}$ is a null ordering period. Thus, this contradicts the fact that $\pi^{\prime}$ is optimal.

Property 3.10. In an optimal plan, for a subplan $(u, v)$ in which the returns are allowed, there is at most one return period and no disposal period. If $t$ is the return period, then, the quantity returned is $q_{t}^{u, v}=\left\lceil\frac{D_{u, v-1}}{V}\right\rceil V-D_{u, v-1}$.

Proof. First, let us prove that there is at most one return period in a subplan in which the returns are allowed. We follow the same first part of the proof of Property 3.1 by changing the quantities $e_{i}^{u, v}$ and $e_{j}^{u, v}$ of disposal in periods $i$ and $j$ (see Section 3.3.1) by the quantities $q_{i}^{u, v}$ and $q_{j}^{u, v}$ of return in periods $i$ and $j^{\prime}$.

Second, let us prove that there is no disposal period in a subplan containing authorized returns. We suppose, in an optimal policy $\pi$, a positive disposed quantity at period $t\left(e_{t}^{u, v}>0\right)$. We decrease $e_{t}^{u, v}$ by $e_{t}^{u, v}$ and increase $q_{t}$ by $e_{t}^{u, v}$ by obtaining a new feasible solution. We notice that the total cost of this new solution will decrease by an amount of $\left(\tilde{p_{t}^{b}}-\tilde{p_{t}^{e}}\right) e_{t}^{u, v}$ because we have in every period $k, \tilde{p_{k}^{b}} \geq \tilde{p_{k}^{e}}$. This is a contradiction of the optimality of $\pi$.

Since there is at most one return and no disposal in $(u, v)$, then the retailer returns all the remaining quantity to the supplier.

There are two possibilities of a subplan $(u, v)$, in which the returns are allowed, according to the position of the return limit $j$ : it can be either with $1 \leq u<v \leq j+1$ or with $1 \leq u \leq j<$ $v \leq T+1$. Let $g$ be the last period permitted for returns in the subplan $(u, v)$ :

$$
g= \begin{cases}v-1 & \text { if } \quad 1 \leq u<v \leq j+1, \text { first possibility } \\ j & \text { if } \quad 1 \leq u \leq j<v \leq T+1, \text { second possibility }\end{cases}
$$

Property 3.11. For a subplan $(u, v)$ in which the returns are allowed $(u \leq g<v)$, the return period coincides with the period having $\max _{i=u, \ldots, g}\left\{\tilde{p}_{i}^{b}\right\}$. If the latter expression is verified for several periods, then the return will be made in the closest period to period $g$.

Proof. First, let us prove that the return period belongs to the set of periods having $\max _{i=u, \ldots, g}\left\{\tilde{p_{i}^{b}}\right\}$. Consider an optimal policy $\pi^{\prime}$ with a return period $t$ between $u$ and $g$ such that $q_{t}^{\prime}>0$ and $t \neq t_{\text {max }}$ in which $\tilde{p}_{t_{\text {max }}}=\max _{i=u, \ldots, g}\left\{\tilde{p}_{i}^{b}\right\}$ (see Figures 3.3 and 3.4 .

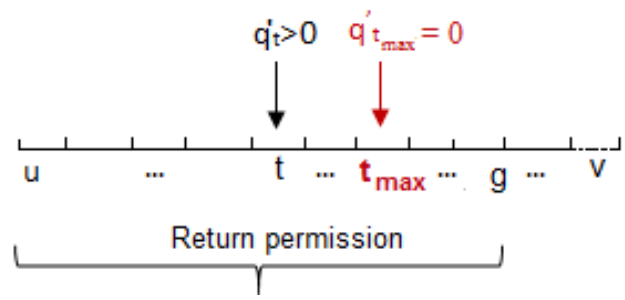

Figure 3.3: A subplan $(u, v)$ having one return Figure 3.4: A subplan $(u, v)$ having one return in period $t<t_{\max }$

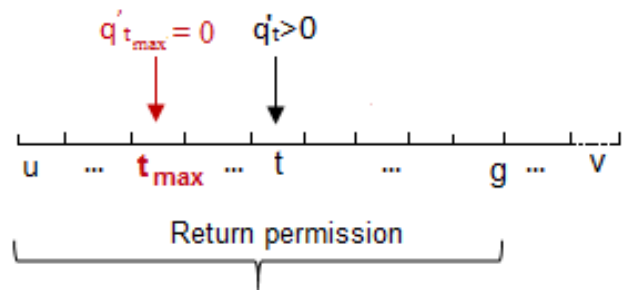

in period $t>t_{\max }$

The period $t_{\max }$ can be either before $t$ or after $t$. Two cases may arise: 
- If $t<t_{\max }$ (Figure 3.3, then we consider a feasible policy $\pi^{\prime \prime}$ which returns $q_{t}^{\prime}$ units less in period $t$, stores $q_{t}^{\prime}$ additional units from period $t$ to period $t_{\text {max }}-1$, and returns $q_{t}^{\prime}$ items more in period $t_{\max }$, that is, $q_{t}^{\prime \prime}=0, s_{k}^{\prime \prime}=s_{k}^{\prime}+q_{k}^{\prime}, \forall k \in\left\{t, \ldots, t_{\max }-1\right\}$ and $q_{t_{\max }}^{\prime \prime}=q_{t}^{\prime}$. The cost of this new policy is: $\mathscr{C}\left(\pi^{\prime \prime}\right)=\mathscr{C}\left(\pi^{\prime}\right)-q_{t}^{\prime}\left(\tilde{p}_{t_{\max }}-\tilde{p}_{t}^{b}\right)$. Since $\pi^{\prime}$ is an optimal policy, we must have $\tilde{p}_{t_{\text {max }}^{b}} \leq \tilde{p_{t}^{b}}$, but it is not the case because we have $\tilde{p}_{t_{\text {max }}}=\max _{i=u, \ldots, t, \ldots, g}\left\{\tilde{p}_{i}^{b}\right\}$ and $t \neq t_{\text {max }}$ - a contradiction of $\pi^{\prime}$.

- If $t>t_{\max }$ (Figure 3.4 , then we define $\lambda=\min \left\{q_{t}^{\prime}, \min _{k=t_{\max }, \ldots, t-1} s_{k}^{\prime}\right\}$ and we consider an alternative policy $\pi^{\prime \prime \prime}$ in which we have $q_{t_{\max }}^{\prime \prime \prime}=\lambda, s_{k}^{\prime \prime \prime}=s_{k}^{\prime}-\lambda, \forall k \in\left\{t_{\max }, \ldots, t-1\right\}$ and $q_{t}^{\prime \prime \prime}=q_{t}^{\prime}-\lambda$. The cost of a policy $\pi^{\prime \prime \prime}$ is computed as follows: $\mathscr{C}\left(\pi^{\prime \prime \prime}\right)=\mathscr{C}\left(\pi^{\prime}\right)-\lambda\left(\tilde{p}_{t_{\max }}-\tilde{p_{t}^{b}}\right)$. A policy $\pi^{\prime}$ is no longer optimal because $\mathscr{C}\left(\pi^{\prime \prime \prime}\right)<\mathscr{C}\left(\pi^{\prime}\right)$. We will prove that, in a policy $\pi^{\prime \prime \prime}$, the return period is chosen among the periods having $\max _{i=u, \ldots, g}\left\{\tilde{p_{i}^{b}}\right\}$ according to the value of $\lambda$. There are two values for $\lambda$ :

- If $\lambda=q_{t}$, then there is one subplan $(u, v)$ and the return is placed in period $t_{\max }$ optimality of $\pi^{\prime \prime \prime}$.

- If $\lambda=\min _{k=t_{\max }, \ldots, t-1} s_{k}^{\prime}=s_{i}^{\prime}$ with $i \in\left\{t_{\max }, \ldots, t-1\right\}$, then the subplan $(u, v)$ is divided into two subplans: $(u, i+1)$ and $(i+1, v)$. In $(u, i+1)$, there is one return in period $t_{\max }$ - the optimality is achieved. For the subplan $(i+1, v)$, we have a return in period $t$. We search for a period being between the periods $i+1$ and $g$ and having the maximum of $\tilde{p}_{i}^{b}$ with $i \in\{i+1, \ldots, g\}$. After that, we perform on $(i+1, v)$ the same procedure of the $(u, v)$ optimality proof till obtaining the $(i+1, v)$ optimality.

If there are several periods having the same maximum of revenue $\tilde{p_{i}^{b}}$ with $i \in\{u, \ldots, g\}$, then, we have to prove that the return period is the last one in the set of possible return periods. Suppose that, in an optimal policy $\pi^{(1)}$, we have in a subplan $(u, v)$ a return period $t_{\text {max }}$ $\left(q_{t_{\text {max }}}^{(1)}>0\right)$ having $\tilde{p}_{t_{\text {max }}}^{b}=\max _{i=u, \ldots, g}\left\{\tilde{p}_{i}^{b}\right\}$. Now, we consider that we have a period $t_{\text {max }_{2}}$ having ${\tilde{p^{b}}}_{t_{\max }}=\max _{i=u, \ldots, g}\left\{\tilde{p_{i}^{b}}\right\}$ with $t_{\max _{1}}<t_{\max _{2}}$. Clearly, there is a replenishment period $t_{1}$ before period $t_{\max _{1}}$, but between $t_{\max _{1}}$ and $t_{\max _{2}}$, we may or may not have some replenishment periods.

In the case where there are no ordering periods, returning units at period $t_{\max _{1}}$ or at period $t_{\max _{2}}$ incurs the same cost for the retailer because they have the same $\tilde{p^{b}}$. For the case where there are ordering periods, we choose one, for example, period $t_{2}$ such that $t_{1} \leq t_{\text {max }_{1}}<t_{2} \leq t_{\text {max }_{2}}$ (see Figure 3.5. According to Property 3.9, we have $\tilde{t_{1}}+\frac{a_{t_{1}}}{V} \geq \tilde{p_{t_{2}}}+\frac{a_{t_{2}}}{V}$ because we have $x_{t_{1}}^{(1)}>0$, $x_{t_{2}}^{(1)}>0$ and $t_{1}<t_{2}$.

Let $\delta_{1}=\min \left\{V, \min _{i=t_{1}, \ldots, t_{\max }-1} s_{i}^{(1)}\right\}$. We have two cases:

- If $\delta_{1}=V$, then we define $\delta_{2}=\min \left\{V-q_{t_{m_{1 x}}}^{(1)}, \min _{i=t_{\max _{1}}, \ldots, t_{2}-1} s_{i}^{(1)}\right\}$ and we obtain two sub-cases: 


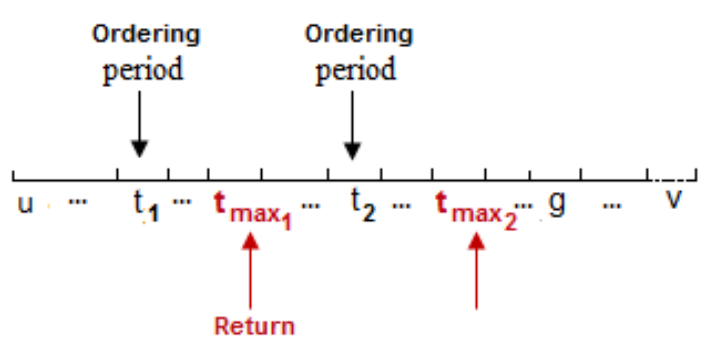

Figure 3.5: A subplan $(u, v)$ having one return in period $t_{\text {max }_{1}}$ in policy $\pi^{(1)}$

- If $\delta_{2}=V-q_{t_{\text {max }}}^{(1)}$, then, we propose a feasible policy $\pi^{(2)}$ in which we obtain the following quantities by changing the ones of policy $\pi^{(1)}: x_{t_{1}}^{(2)}=x_{t_{1}}^{(1)}-V, s_{i}^{(2)}=s_{i}^{(1)}-\delta_{1}$, $\forall i \in\left\{t_{1}, \ldots, t_{\text {max }_{1}}-1\right\}, q_{t_{\text {max }}}^{(2)}=0, s_{i}^{(2)}=s_{i}^{(1)}-\delta_{2}, \forall i \in\left\{t_{\max _{1}}, \ldots, t_{2}-1\right\}, x_{t_{2}}^{(2)}=$ $x_{t_{2}}^{(1)}+V, s_{i}^{(2)}=s_{i}^{(1)}+q_{t_{\max }}^{(1)}, \forall i \in\left\{t_{2}, \ldots, t_{\max _{2}}-1\right\}$ and $q_{t_{\max }}^{(2)}=q_{t_{\max }}^{(1)}$. The total cost $\mathscr{C}\left(\pi^{(2)}\right)$ is computed as a function of the total cost $\mathscr{C}\left(\pi^{(1)}\right)$ as follows: $\mathscr{C}\left(\pi^{(2)}\right)=$ $\mathscr{C}\left(\pi^{(1)}\right)-V\left(\left(\tilde{p_{t_{1}}}+\frac{a_{t_{1}}}{V}\right)-\left(\tilde{p_{t_{2}}}+\frac{a_{t_{2}}}{V}\right)\right)$. Hence, we observe that $\mathscr{C}\left(\pi^{(2)}\right) \leq \mathscr{C}\left(\pi^{(1)}\right)-$ this proves the non-optimality of policy $\pi^{(1)}$ if we have $\delta_{1}=V$ and $\delta_{2}=V-q_{t_{\text {max }}}^{(1)}$.

- If $\delta_{2}=\min _{i=t_{\max }, \ldots, t_{2}-1} s_{i}^{(1)}$, then the retailer has the possibility to return either in period $t_{\text {max }_{1}}$ or in period $t_{\text {max }_{2}}$, since the cost of these two policies are equal.

- If $\delta_{1}=\min _{i=t_{1}, \ldots, t_{\max }-1} s_{i}^{(1)}$, then the returning in period $t_{\text {max }}$ is optimal as the returning in period $t_{\text {max }_{1}}$ of policy $\pi^{(1)}$.

Property 3.12. In an optimal solution, for the subplan $(u, v)$ with $j<u<v \leq T+1$, there is at most one disposal period, situated in period $u$ with $e_{u}^{u, v}=\left\lceil\frac{D_{u, v-1}}{V}\right\rceil V-D_{u, v-1}$ and no return period.

After period $j$, the returns are not allowed, refer to the proof of Property 3.1 and 3.2.

An optimal subplan $(u, v)$ with $j<u<v \leq T+1$ verifies Property 3.4 in which we can identify the ending inventory value of each ordering period $k$.

Property 3.13. For a subplan $(u, v)$ in which the return is placed in period $t\left(q_{t}^{u, v} \geq 0\right)$, there exists an optimal schedule which verifies for each ordering period $k$ :

$\forall k \in\{u, \ldots, t\}, s_{k-1}=R_{u, k-1}$

$\forall k \in\{t+1, \ldots, v\}, s_{k-1}=R_{u, k-1}-q_{t}^{u, v}+\mathbb{1}_{R_{u, k-1}<q_{t}^{u, v}} V$.

The proof of Property 3.13 is the same as that of Property 3.4.

Computation of $\mathscr{C}(u, v)$ :

As it was noticed in the properties, there are two types of suplan $(u, v)$ whose optimal costs are computed as follows: 
In $(u, v)$ with $j<u<v \leq T+1$, by using Properties 3.4 and 3.12 , the optimal cost $\mathscr{C}(u, v)$ is equal to $\mathscr{C}_{v}^{(1)}(u, v)$ as explained in Section 3.3.1.

In $(u, v)$ with either $1 \leq u<v \leq j+1$ or $1 \leq u \leq j<v \leq T+1$, by using Properties 3.10, 3.11 and 3.13 , the optimal cost $\mathscr{C}(u, v)$ is equal to $\mathscr{C}_{v}^{(3)}(u, v)$ (see the following). The cost $\mathscr{C}_{v}^{(3)}(u, l+1)$ is the minimum cost of satisfaction of $d_{u}, d_{u+1}, \ldots, d_{l}$, knowing that the subplan $(u, v)$ contains one return in period $t$, and period $l+1$ is a replenishment period with $1 \leq u \leq l \leq v-1 \leq T$. The dynamic programming formulation of $\mathscr{C}_{v}^{(3)}(u, l+1)$ is as follows:

Recurrence relation. For $l=u, \ldots, v-1$,

$$
\begin{aligned}
\mathscr{C}_{v}^{(3)}(u, l+1)=\min _{u-1 \leq k<l}\{ & \mathscr{C}_{v}^{(3)}(u, k+1)+ \\
& f_{k+1}+\left(p_{k+1}+\frac{a_{k+1}}{V}\right)\left(s_{l}+D_{k+1, l}+\mathbb{1}_{k+1 \leq t<l+1} q_{t}^{u, v}-s_{k}\right)+ \\
& \left.H_{u, v}^{(3)}(k+1, l+1)-\mathbb{1}_{k+1 \leq t<l+1} p_{t}^{b} q_{t}^{u, v}\right\}
\end{aligned}
$$

Boundary condition. $\mathscr{C}_{v}^{(3)}(u, u)=0$

Objective. $\mathscr{C}_{v}^{(3)}(u, v)$

To compute the cost $\mathscr{C}_{v}^{(3)}(u, l+1)$, we have to ensure that all the periods $k+1$ are replenishment periods just before period $l+1$. The number of batches purchased in period $k+1$ satisfies the demands of periods $k+1, k+2, \ldots, l$ and also the returned quantity $q_{t}^{u, v}$ if $k+1 \leq t<l+1$ by taking into account the ending inventory levels of periods $k$ and $l$ given in Property 3.13 according to the position of period $t$. In addition to the ordering cost, we have the total holding cost $H_{u, v}^{(3)}(k+1, l+1)$ from the end of period $k+1$ up to the beginning of period $l+1$ knowing that there are no orders between $k+2$ and $l$, we can compute the value of $s_{l}$ and if $k+1<t<l+1$ we also have to store $q_{t}^{u, v}$. All the values of $H_{u, v}^{(3)}(k+1, l+1)$ can be calculated recursively in $O\left(T^{5}\right)$ time, knowing that the complexity for finding the maximum of $\tilde{p_{i}^{b}}$ in order to obtain the return period $t$ is $O(T)$,

$$
\begin{cases}H_{u, v}^{(3)}(k+1, l+1)=\left(H_{u, v}^{(3)}(k+2, l+1)+\right. & \text { if } u-1 \leq k<l \leq v-1 \leq T \\ \left.h_{k+1}\left(D_{k+2, l}+s_{l}+\mathbb{1}_{k+1<t<l+1} q_{t}^{u, v}\right)\right) & \text { and } \mathbb{1}_{l \neq v-1 \text { or } k \neq v-2}=1 \\ H_{u, v}^{(3)}(l+1, l+1)=0 & \text { if } u \leq l \leq v-1 \leq T \\ H_{u, v}^{(3)}(v-1, v)=0 & \text { if } 2 \leq v \leq T+1\end{cases}
$$

In the relation of $\mathscr{C}_{v}^{(3)}(u, l+1)$, we deduce all the costs mentioned above by the return revenues in period $t$ if $k+1 \leq t<l+1$. One can compute all the values of $\mathscr{C}_{v}^{(3)}(u, v)$ with a complexity of $O\left(T^{5}\right)$.

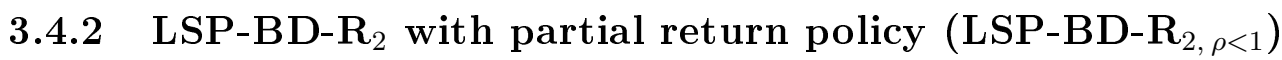

For this case with $\rho<1$, we assume that the quantities returned over the first $j$ periods are restricted by the quantity $\rho \sum_{i=1}^{j} x_{i}$. We will prove that a special case of our problem with $j=T$ and $\max _{i=1, \ldots, j}\left\{\tilde{p_{i}^{b}}\right\} \leq \tilde{p}_{t}, \forall t \in\{1, \ldots, j\}$ can be solved using Weight Constrained Shortest Path Problem (WCSPP). After that, we show that the general problem is like the Resource Constrained Shortest Path Problem (RCSPP). 
LSP-BD- $\mathbf{R}_{2, \rho<1}$ for the special case with $j=T$ and $\max _{i=1, \ldots, j}\left\{\tilde{p_{i}^{b}}\right\} \leq \tilde{p}_{t}, \forall t \in\{1, \ldots, j\}$

The assumption of $j=T$ means that the retailer can return units in every period of the planning horizon. We first identify some optimality properties of this case to calculate all the possible costs of the subplan $(u, v)$.

Some preliminaries and properties:

Property 3.14. There exists an optimal solution in which for any subplan $(u, v)$ such that $1 \leq u<v \leq T+1$, there is at most one disposal in $u$ and one return situated in the last period $t$ having $\max _{i=u, \ldots, v-1}\left\{\tilde{p}_{i}^{b}\right\}$. We have $e_{u}^{u, v}+q_{t}^{u, v}=\left\lceil\frac{D_{u, v-1}}{V}\right\rceil V-D_{u, v-1}$.

Proof. The fact that there is just one return period in a $(u, v)$ is proved in Property 3.10. However, we can have either only one disposal period, or, one disposal period and one return period, because, all the optimal subplans must verify the buyback constraint $\left(\sum_{i=1}^{T} q_{i} \leq \rho \sum_{i=1}^{T} x_{i}\right)$. All the quantities one may want to return can be limited by this latter upper bound. In a subplan with a null remaining quantity $\left(\left\lceil\frac{D_{u, v-1}}{V}\right\rceil V-D_{u, v-1}=0\right)$, there is neither return nor disposal. See the proof of Property 3.11 in order to find the optimal return period and the one of Property 3.1 in order to find the disposal period.

Property 3.15. For any subplan $(u, v)$ with $e_{u}^{u, v} \geq 0, q_{t}^{u, v} \geq 0$ and $t$ being the optimal return period, there exists an optimal schedule which verifies for each replenishment period $k$ :

$\forall k \in\{u+1, \ldots, t\}, s_{k-1}=R_{u, k-1}-e_{u}^{u, v}+\mathbb{1}_{R_{u, k-1}<e_{u}^{u, v} V}$

$\forall k \in\{t+1, \ldots, v\}, s_{k-1}=R_{u, k-1}-q_{t}^{u, v}-e_{u}^{u, v}+\mathbb{1}_{R_{u, k-1}<q_{t}^{u, v}+e_{u}^{u, v}} V$.

It is a consequence of Property 3.14.

Property 3.16. The optimal purchased quantity, throughout the planning horizon, is equal to $\left\lceil\frac{D_{1, T}}{V}\right\rceil V$.

Therefore, the retailer has to meet only the demands $d_{1}, d_{2}, \ldots, d_{T}$. By satisfying the buyback constraint on the total quantity returned, we obtain $\sum_{i=1}^{T} q_{i} \leq \rho\left\lceil\frac{D_{1, T}}{V}\right\rceil V$ and subsequently $\sum_{i=1}^{T} q_{i} \leq W$ with $W=\left\lfloor\rho\left\lceil\frac{D_{1, T}}{V}\right\rceil V\right\rfloor$.

Proof. The proof of this property is similar to that of Property 3.8 but with some modifications, because the returns are allowed in every period. We suppose an optimal policy $\pi^{\prime}$ with a total number of batches replenished being $\left\lceil\frac{D_{1, T}}{V}\right\rceil V+V$. To obtain an alternative policy $\pi^{*}$ in which the retailer only purchases $\left\lceil\frac{D_{1, T}}{V}\right\rceil V$ items, we decrease the disposal quantity and the quantity returned of the policy $\pi^{\prime}$ by the batch size $V$. We detect the periods where we have $e_{t}^{\prime}>0$, for example $t_{1}$ and $t_{2}$, and the ones with $q_{t}^{\prime}>0$, for example $t_{3}$ and $t_{4}$. We suppose first that we have $t_{1}<t_{2}<t_{3}<t_{4}$, knowing that our procedure works with any compositions of these periods, and, second that $t_{5}$ is an ordering period placed after $t_{1}, t_{2}, t_{3}$ and $t_{4}$. We choose four integers: $\alpha_{1}, \alpha_{2}, \beta_{3}$ and $\beta_{4}$ such that $\alpha_{1} \leq e_{t_{1}}^{\prime}, \alpha_{2} \leq e_{t_{2}}^{\prime}, \beta_{3} \leq q_{t_{3}}^{\prime}, \beta_{4} \leq q_{t_{4}}^{\prime}, V=\alpha_{1}+\alpha_{2}+\beta_{3}+\beta_{4}$, $\sum_{t=1}^{T} q_{t}^{\prime}-\rho\left\lceil\frac{D_{1, T}}{V}\right\rceil V \leq \beta_{3}+\beta_{4}$ and $\alpha_{1}, \alpha_{2}, \beta_{3}, \beta_{4} \in \mathbb{N}$.

The policy $\pi^{*}$ gets rid of $e_{t_{i}}^{\prime}-\alpha_{i}$ in period $t_{i}$, for $i=1,2$. Besides, it returns $q_{t_{k}}^{\prime}-\beta_{k}$ in period $t_{k}$, for $k=3$, 4. In addition, it stores $s_{c}^{\prime}+\alpha_{1}$ from period $t_{1}$ till $t_{2}-1, s_{c}^{\prime}+\alpha_{1}+\alpha_{2}$ from period $t_{2}$ till $t_{3}-1, s_{c}^{\prime}+\alpha_{1}+\alpha_{2}+\beta_{3}$ from period $t_{3}$ till $t_{4}-1$, and, $s_{c}^{\prime}+\alpha_{1}+\alpha_{2}+\beta_{3}+\beta_{4}$ from period $t_{4}$ till $t_{5}-1$. Finally, it orders $x_{t_{5}}^{\prime}-V$ in $t_{5}$. Then, we obtain:

$$
\mathscr{C}\left(\pi^{*}\right)=\mathscr{C}\left(\pi^{\prime}\right)-\left(V\left(\tilde{p_{t_{5}}}+\frac{a_{t_{5}}}{V}\right)-\alpha_{1} \tilde{p}_{t_{1}}^{\tilde{e}}-\alpha_{2} p_{t_{2}}^{\tilde{e}}-\beta_{3} \tilde{p}_{t_{3}}^{\tilde{b}}-\beta_{4} \tilde{p}_{t_{4}}^{\tilde{b}}\right)
$$


Since we have $\max _{i=1, \ldots, T}\left\{\tilde{p_{i}^{b}}\right\} \leq \tilde{p_{t}}, \tilde{p_{t}^{e}} \leq \tilde{p_{t}^{b}}, \forall t \in\{1, \ldots, T\}$, and, $V=\alpha_{1}+\alpha_{2}+\beta_{3}+\beta_{4}$, thus we note that the cost of policy $\pi^{*}$ is lower than the one of policy $\pi^{\prime}$ - contradiction of the optimality of the policy $\pi^{\prime}$.

The optimal cost of LSP-BD-R $\mathrm{R}_{2, \rho<1}$ for the special case with $j=T$ and $\max _{i=1, \ldots, j}\left\{\tilde{p_{i}^{b}}\right\} \leq \tilde{p}_{t}$, $\forall t \in\{1, \ldots, j\}$ will match with $\sum_{i=1}^{T} x_{i}=\left\lceil\frac{D_{1, T}}{V}\right\rceil V$.

Computation of the possible costs of the subplan $(u, v)$ :

According to Property 3.14, the value of $\rho$ influences the costs of all the possible subplans. We cannot independently compute the optimal cost $\mathscr{C}(u, v)$ for a subplan $(u, v)$ (see Example 3.4). The reason is that, even if in each subplan $(u, v)$, we know the value of the remaining units which will be divided into a returned quantity and a disposed amount, we cannot easily determine those latters because of the buyback constraint $\sum_{i=1}^{T} q_{i} \leq W$ linking all the subplans of a feasible plan. A decision already made on a return quantity in a previous subplan will heavily impact the return quantities in the next subplans. In the following, we will show that this problem is similar to WCSPP.

Example 3.4. We take Example 3.3 for $j=T=3$ and we change the unit procurement cost to be 13 in every period in order to verify $\max _{i=1, \ldots, 3}\left\{\tilde{p_{i}^{b}}\right\} \leq \tilde{p}_{t}, \forall t \in\{1, \ldots, 3\}$. In Table 3.4 we see the results of LSP-BD- $R_{2, \rho=\frac{1}{5}}$ and LSP-BD-R $2, \rho=\frac{1}{10}$. We note that for the case where we have $\rho=\frac{1}{5}$, the cost of the subplan $(1,4)$ in the optimal plan coincides with the optimal cost $\mathscr{C}_{4}^{(3)}(1,4)$. However, for the case where we have $\rho=\frac{1}{10}$, the cost of the subplan $(1,4)$ in the optimal plan will be higher because of the buyback constraint to respect.

Table 3.4: The optimal quantities of LSP-BD-R ${ }_{2, \rho=\frac{1}{5}}$ and LSP-BD- $\mathrm{R}_{2, \rho=\frac{1}{10}}$ for $j=3$

\begin{tabular}{|c|c|c|c|c|c|c|c|c|}
\hline$V=4$ & \multicolumn{4}{|c|}{ LSP-BD-R $_{2, \rho=\frac{1}{5}} j=3$} & \multicolumn{4}{|c|}{ LSP-BD-R $_{2, \rho=\frac{1}{10}} j=3$} \\
\hline Period $t$ & $x_{t}$ & $s_{t}$ & $q_{t}$ & $e_{t}$ & $x_{t}$ & $s_{t}$ & $q_{t}$ & $e_{t}$ \\
\hline 1 & 4 & 2 & 1 & 0 & 4 & 2 & 0 & 1 \\
\hline 2 & 0 & 1 & 0 & 0 & 0 & 1 & 0 & 0 \\
\hline 3 & 0 & 0 & 0 & 0 & 0 & 0 & 0 & 0 \\
\hline Costs & \multicolumn{4}{|c|}{70} & \multicolumn{4}{|c|}{71} \\
\hline
\end{tabular}

We have $R_{u, v-1}+1$ possible costs of a subplan $(u, v)$, denoted by $\mathscr{F}_{\phi}^{(v)}(u, v)$ for $\phi=$ $0,1, \ldots, R_{u, v-1}$. Note that the quantity $R_{u, v-1}$ represents the unused items in a subplan $(u, v)$ with $R_{u, v-1}=\left\lceil\frac{D_{u, v-1}}{V}\right\rceil V-D_{u, v-1}$. In order to find the cost $\mathscr{F}_{\phi}^{(v)}(u, v)$, we satisfy $D_{u, v-1}$, we get rid of $R_{u, v-1}-\phi$ units at period $u$ and we return $\phi$ units at the optimal return period $t$. For $\phi=0$, the first possible cost $\mathscr{F}_{0}^{(v)}(u, v)$ is equal to the cost $\mathscr{C}_{v}^{(1)}(u, v)$ in which there is only one disposal of $R_{u, v-1}$ units. For $1 \leq \phi \leq R_{u, v-1}-1$, there are $R_{u, v-1}-1$ possible costs with both one return and one disposal. In this case, the dynamic programming formulation of $\mathscr{F}_{\phi}^{(v)}(u, v)$ is the combination of the ones of $\mathscr{C}_{v}^{(1)}(u, v)$ and $\mathscr{C}_{v}^{(3)}(u, v)$. For $\phi=R_{u, v-1}$, the last possible cost coincides with the cost $\mathscr{C}_{v}^{(3)}(u, v)$ with one return of $R_{u, v-1}$ units and zero disposal. For each subplan $(u, v)$ with $1 \leq u<v \leq T+1$, we can compute at most $V$ possible costs because the remaining units are less than $V\left(R_{u, v-1} \leq V-1\right)$. 
Modeling LSP-BD-R $R_{2, \rho<1}$ for the special case with $j=T$ and $\max _{i=1, \ldots, j}\left\{\tilde{p}_{i}^{b}\right\} \leq \tilde{p}_{t}, \forall t \in$ $\{1, \ldots, j\}$ as $W C S P P$ :

We consider a network $G=(I, E)$ where $I$ represents the set of nodes $\{1, \ldots, T+1\}$ and $E$ the set of $\operatorname{arcs}\left\{(u, v)_{\phi} \mid u \in I, v \in I, \phi=0, \ldots, R_{u, v-1}, u<v\right\}$. For each $\operatorname{arc}(u, v)_{\phi} \in I$, we associate two non-negative weights, $\mathscr{F}_{\phi}^{(v)}(u, v)$ and $\phi$, being respectively, the cost and the return quantity incurred by traversing the arc. Our problem consists in finding a path in $G$ from node 1 to node $T+1$ that minimizes the total cost, subject to not exceeding the given upper bound $W \in \mathbb{N}$ representing the total returned quantity (see Property 3.16). In $G$, between two nodes $u$ and $v$, there are $R_{u, v-1}+1$ arcs.

Now, we will transform the graph $G$ into another one having only one arc between any two given nodes. First, we replace each node $u$ such that $u \in I \backslash\{T+1\}$ by a number of nodes denoted by $n b r_{u}$ in the new network $G^{\prime}$ with $n b r_{u}=\max _{v \in I, u<v}\left\{R_{u, v-1}+1\right\}$. The node $T+1$ is replaced with one node. Therefore, in $G^{\prime}$, we have $\sum_{t=1}^{T} n b r_{u}+1$ nodes. We consider the new set of nodes $I^{\prime}$ with $I^{\prime}=\left\{1, \ldots, \sum_{t=1}^{T} n b r_{u}+1\right\}$. In $G$, for $u \in I, v \in I$ with $u<v$, there exist several arcs between these two nodes. We suppose that the node $u$ is replaced with $k$ nodes in $G^{\prime}: u_{1}, u_{2}, \ldots, u_{k}$ and the node $v$ is replaced with $i$ nodes in $G^{\prime}: v_{1}, v_{2}, \ldots, v_{i}$ with $u_{1}<u_{2}<\cdots<u_{k}<v_{1}<v_{2}<\cdots<v_{i}$. We consider in $G^{\prime}$ the set of arcs $E^{\prime}$ with $E^{\prime}=\left\{\left(u^{\prime}, v^{\prime}\right) \mid u^{\prime} \in I^{\prime}, v^{\prime} \in I^{\prime}, u^{\prime}<v^{\prime}\right\}$. We will compute two non-negative weights for each link $\left(u^{\prime}, v^{\prime}\right): F\left(u^{\prime}, v^{\prime}\right)$ being its associated cost and $q^{u^{\prime}, v^{\prime}}$ being its associated return quantity:

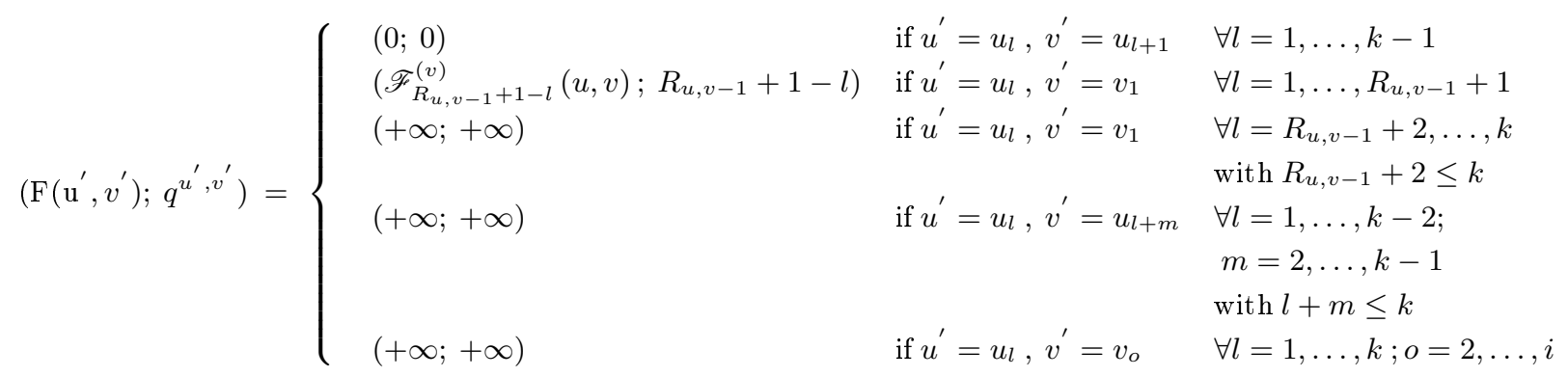

We give the graph $G$ for the Example 3.4, where we have 4 nodes: 1, 2, 3 and 4 representing the periods of the planning horizon $1 . .3$ and the dummy period 4 (see Figure 3.6). For instance, between node 1 and node 2 , there are 4 arcs: $(1,2)_{0},(1,2)_{1},(1,2)_{2}$ and $(1,2)_{3}$ because the remaining items $R_{1,1}$ of the subplan $(1,2)$ is equal to 3 . For each arc, we compute the values of the cost and the return quantity.

Now, we will draw the transformed graph $G^{\prime}$ stated in Appendix. The node 1 is replaced with 4 nodes in the network $G^{\prime}: 1^{\prime}, 2^{\prime}, 3^{\prime}, 4^{\prime}$, since $n b r_{1}=4$. The same transformation is made for nodes 2 and 3 . The last node 4 is replaced with one node being $13^{\prime}$. Therefore, in $G^{\prime}$, we have 13 nodes. To understand how to put the values of $F\left(u^{\prime}, v^{\prime}\right)$ and $q^{u^{\prime}, v^{\prime}}$ in each arc of the network $G^{\prime}$, we take, for example, node $u=1$ in $G$ being replaced in $G^{\prime}$ with $u_{1}=1^{\prime}, u_{2}=2^{\prime}, u_{3}=3^{\prime}$ and $u_{4}=4^{\prime}(k=4)$ and node $v=2$ being replaced in $G^{\prime}$ with $v_{1}=5^{\prime}, v_{2}=6^{\prime}, v_{3}=7^{\prime}$ and $v_{4}=8^{\prime}(i=4)$. By replacing $k$ with 4 in the first equation of $\left(F\left(u^{\prime}, v^{\prime}\right) ; q^{u^{\prime}, v^{\prime}}\right)$, we find null values of cost and return quantity for the following arcs: $\left(1^{\prime}, 2^{\prime}\right),\left(2^{\prime}, 3^{\prime}\right),\left(3^{\prime}, 4^{\prime}\right)$. For $l=1, \ldots, 4$, the $\operatorname{arc}\left(u_{l}, 5^{\prime}\right)$ has the cost $\mathscr{F}_{4-l}^{(2)}(1,2)$ and the quantity returned $4-l$. For $l=1,2$ and $m=2,3$ 
with $l+m \leq 4$, we obtain the $\operatorname{arcs}\left(u_{l}, u_{l+m}\right)$ having infinite costs and return quantities: $\left(1^{\prime}, 3^{\prime}\right)$, $\left(1^{\prime}, 4^{\prime}\right)$ and $\left(2^{\prime}, 4^{\prime}\right)$. For $l=1, \ldots, 4$ and $o=2, \ldots, 4$, we find infinite costs and return quantities in the $\operatorname{arcs}\left(u_{l}, v_{o}\right):\left(1^{\prime}, 6^{\prime}\right),\left(1^{\prime}, 7^{\prime}\right),\left(1^{\prime}, 8^{\prime}\right),\left(2^{\prime}, 6^{\prime}\right),\left(2^{\prime}, 7^{\prime}\right),\left(2^{\prime}, 8^{\prime}\right),\left(3^{\prime}, 6^{\prime}\right),\left(3^{\prime}, 7^{\prime}\right),\left(3^{\prime}, 8^{\prime}\right),\left(4^{\prime}, 6^{\prime}\right)$, $\left(4^{\prime}, 7^{\prime}\right),\left(4^{\prime}, 8^{\prime}\right)$. We did not draw the arcs having infinite costs to avoid having a congested graph. Thus, if there is no arc between two nodes in Figure 7.1. then its cost and its return quantity are infinite.

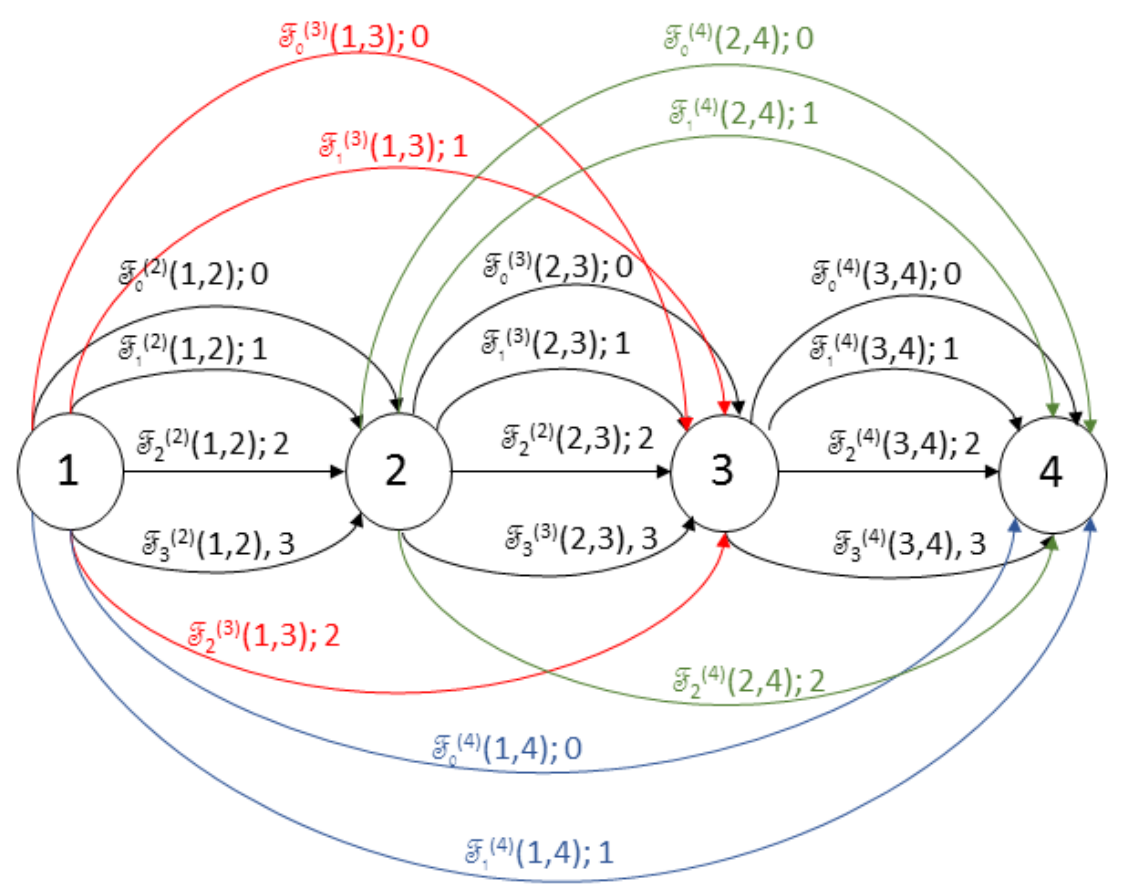

Figure 3.6: Graph $G$ of Example 3.4

After showing that the graph $G$ of our problem having at most $V$ arcs between two nodes can be represented by an equivalent network $G^{\prime}$ having one arc between two nodes, we present our problem as finding a minimum cost feasible path from node 1 to node $\sum_{t=1}^{T} n b r_{u}+1$ in $G^{\prime}$. A path is feasible if and only if the total cumulated quantity returned along the path is at most $W$. Our problem can be formulated as the following integer linear program:

$$
\begin{aligned}
& \min \sum_{\left(u^{\prime}, v^{\prime}\right) \in E^{\prime}} F\left(u^{\prime}, v^{\prime}\right) X_{u^{\prime}, v^{\prime}} \\
& \text { s.t. } \\
& \sum_{v^{\prime} \mid\left(u^{\prime}, v^{\prime}\right) \in E^{\prime}} X_{u^{\prime}, v^{\prime}}-\sum_{v^{\prime} \mid\left(v^{\prime}, u^{\prime}\right) \in E^{\prime}} X_{v^{\prime}, u^{\prime}}= \begin{cases}1 & u^{\prime}=1 \\
-1 & u^{\prime}=\sum_{t=1}^{T} n b r_{u}+1 \quad \forall u^{\prime} \in I^{\prime} \\
0 & \text { otherwise }\end{cases} \\
& \sum_{\left(u^{\prime}, v^{\prime}\right) \in E^{\prime}} q^{u^{\prime}, v^{\prime}} X_{u^{\prime}, v^{\prime}} \leq W \\
& X_{u^{\prime}, v^{\prime}} \in\{0,1\} \\
& \forall\left(v^{\prime}, u^{\prime}\right) \in E^{\prime}
\end{aligned}
$$


The above formulation shows that the LSP-BD-R $2, \rho<1$ with $j=T$ and $\max _{i=1, \ldots, T}\left\{\tilde{p_{i}^{b}}\right\} \leq \tilde{p}_{t}$, $\forall t \in\{1, \ldots, T\}$ is a Weight-Constrained Shortest Path Problem (WCSPP) which consists in searching the least cost path from a start node to a final node, such that the total weight of one resource is limited by a specified integer. This problem has been shown to be NP-hard even for acyclic networks (Garey and Johnson, 1979). If there is a directed graph with non-negative cost cycles (like the graph of our problem), then the WCSPP becomes NP-hard in the weak sense and it can be solved by pseudo-polynomial time algorithms. There are two categories of methods developed to solve the WCSPP. There are exact approaches based on $\mathrm{k}$ shortest paths (Eppstein, 1997), node labeling methods derived from dynamic programming equations (Zhu and Wilhelm, 2012), and Lagrangian relaxation (Carlyle et al., 2008). Besides, we also find approximation algorithms based on scaling and rounding of data (Lorenz and Raz, 2001).

\section{LSP-BD-R $\mathbf{R}_{2, \rho<1}$ for the general case}

In the general case, we cannot identify the optimal total quantity purchased $\sum_{i=1}^{j} x_{i}$ from period 1 to period $j$, but we can determine its lower and upper bounds by using the following property.

Property 3.17. In an optimal policy, the quantity ordered in periods $1,2, \ldots, j$ can be either $\left\lceil\frac{D_{1, j}}{V}\right\rceil V$, or $\left(\left\lceil\frac{D_{1, j}}{V}\right\rceil+1\right) V$, or $\ldots$, or $\left(\left\lceil\frac{D_{1, T}}{V}\right\rceil+j-1\right) V$, which means that we have $\left\lceil\frac{D_{1, j}}{V}\right\rceil V \leq$ $\sum_{i=1}^{j} x_{i} \leq\left(\left\lceil\frac{D_{1, T}}{V}\right\rceil+j-1\right) V$. For each possible value of $\sum_{i=1}^{j} x_{i}$, we can determine the upper limit of $\sum_{i=1}^{j} q_{i}$.

Proof. The lower bound is obtained by the minimum quantity satisfying only the demands from period 1 to period $j,\left\lceil\frac{D_{1, j}}{V}\right\rceil V$. However, to calculate the upper bound, we have to consider, on the one hand, the path from node 1 to node $j$ with the maximum number of subplans: $(1,2),(2,3),,(j-1, j)$, and on the other hand, the possible maximum procurement at period $j$ to cover the demands of periods $j, \ldots, T$. Hence, the upper limit is equal to $\left(\sum_{i=1}^{j-1}\left\lceil\frac{d_{i}}{V}\right\rceil+\left\lceil\frac{D_{j, T}}{V}\right\rceil\right) V$ which is less than $\left(\left\lceil\frac{D_{1, T}}{V}\right\rceil+j-1\right) V$.

Now, we will show that we can construct a graph for the general LSP-BD- $R_{2, \rho<1}$ which is the same graph $G$ of the LSP-BD-R $2, \rho<1$ with $j=T$ and $\max _{i=1, \ldots, T}\left\{\tilde{p_{i}^{b}}\right\} \leq \tilde{p_{t}}, \forall t \in\{1, \ldots, T\}$, by adding a third non-negative weight for each $\operatorname{arc}(u, v)_{\phi}$ in addition to the cost $\mathscr{F}_{\phi}^{(v)}(u, v)$ and the returned quantity $\phi$ which are easily recalculated for the general case according to Properties $3.11,3.12$ and 3.15. We recall that if we have $j<u<v \leq T+1$, then there is no return in the subplan $(u, v)$ and then $\phi$ receives a zero value. The third weight, denoted by $\psi_{\phi}(u, v)$, of the subplan $(u, v)$ with $\phi$ units of returned quantity, is the optimal total quantity purchased in periods in which returns are allowed. We can obtain this quantity after the calculation of the $\operatorname{cost} \mathscr{F}_{\phi}^{(v)}(u, v)$ by which we get the optimal planning of the subplan $(u, v)$ with $\phi$ units of return, then we get:

$$
\psi_{\phi}(u, v)= \begin{cases}\left(\sum_{i=u}^{v-1} x_{i}\right)_{(u, v)_{\phi}} & 1 \leq u<v \leq j+1 \\ \left(\sum_{i=u}^{j} x_{i}\right)_{(u, v)_{\phi}} & 1 \leq u \leq j<v \leq T+1 \\ 0 & j<u<v \leq T+1\end{cases}
$$

The transformation procedure of the graph of this general problem is the same one of the special case, explained in the previous section, by associating for each link $\left(u^{\prime}, v^{\prime}\right), \psi^{u^{\prime}, v^{\prime}}$ being its total quantity purchased during periods when returns are allowed in addition to the cost $F\left(u^{\prime}, v^{\prime}\right)$ 
and the return quantity $q^{u^{\prime}, v^{\prime}}$. The quantity $\psi^{u^{\prime}, v^{\prime}}$ can take the following values according to the positions of nodes $u^{\prime}$ and $v^{\prime}$ detailed in the equations of $\left(F\left(u^{\prime}, v^{\prime}\right), q^{u^{\prime}, v^{\prime}}\right)$ in the previous subsection: $0, \psi_{R_{u, v-1}+1-l}(u, v),+\infty,+\infty,+\infty$.

For a possible value of $\sum_{i=1}^{j} x_{i}$, denoted by $M$ for example, we present the problem as finding the minimum cost of a path from node 1 to the last node of the transformed network $G^{\prime}$ such that the total quantity purchased from period 1 to period $j$ is equal to $M$ and the total return amount along the path is at most $\rho M\left(=W^{\prime}\right)$. The LSP-BD- $\mathrm{R}_{2, \rho<1}$ with $M$ ordering amount throughout the periods $1,2, \ldots, j$ can be formulated as the following integer linear program:

$$
\begin{aligned}
& \min \sum_{\left(u^{\prime}, v^{\prime}\right) \in E^{\prime}} F\left(u^{\prime}, v^{\prime}\right) X_{u^{\prime}, v^{\prime}} \\
& \text { s.t. } \\
& \qquad \sum_{v^{\prime} \mid\left(u^{\prime}, v^{\prime}\right) \in E^{\prime}} X_{u^{\prime}, v^{\prime}}-\sum_{v^{\prime} \mid\left(v^{\prime}, u^{\prime}\right) \in E^{\prime}} X_{v^{\prime}, u^{\prime}}=\left\{\begin{array}{l}
1 \\
-1 \\
0
\end{array} \begin{array}{l}
u^{\prime}=1 \\
\text { otherwise }
\end{array} \sum_{t=1}^{T} n b r_{u}+1 \quad \forall u^{\prime} \in I^{\prime}\right. \\
& \sum_{\left(u^{\prime}, v^{\prime}\right) \in E^{\prime}} \psi^{u^{\prime}, v^{\prime}} X_{u^{\prime}, v^{\prime}}=M \\
& \sum_{\left(u^{\prime}, v^{\prime}\right) \in E^{\prime}} q^{u^{\prime}, v^{\prime}} X_{u^{\prime}, v^{\prime}} \leq W^{\prime} \\
& X_{u^{\prime}, v^{\prime}} \in\{0,1\}
\end{aligned}
$$

Hence, this problem is similar to a resource constrained shortest path problem (RCSPP) in which we have two resources: ordering quantity and return quantity. The RCSPP is a generalization of the WCSPP because there is a finite set of resources. The consumption of the resource 'ordering quantity' is expressed by an equality constraint, while the consumption of the resource 'return quantity' is expressed by an inequality constraint. This problem can also be modeled as an RCSPP with the following double sided inequality constraints $\left(M \leq \sum_{\left(u^{\prime}, v^{\prime}\right) \in E^{\prime}} \psi^{u^{\prime}, v^{\prime}} X_{u^{\prime}, v^{\prime}} \leq M\right.$, and $\left.0 \leq \sum_{\left(u^{\prime}, v^{\prime}\right) \in E^{\prime}} q^{u^{\prime}, v^{\prime}} X_{u^{\prime}, v^{\prime}} \leq W^{\prime}\right)$.

There are several variants of RCSPP: with upper bound resource limitations (RCSPPI), with equality resource limitations (RCSPPE), with a window for each of several types of resources at each node, with a window for one type of resource (e.g., time) at each node, a combination of these latter, etc. The RCSPPI with a fixed number of resources and an acyclic graph without negative costs is weakly NP-hard and it is widely studied in the literature. For detailed monographs on these problems, refer to Ziegelmann (2001), Zhu (2005) and Garcia (2009). In contrast, there are only few research papers on the RCSPPE (see for example Zhu and Wilhelm, 2007; Turner, 2011). Besides, the RCSPP with lower and upper resource limits was not addressed so much in the literature. It is studied by Ribeiro and Minoux (1985) for a single resource, and by Beasley and Christofides (1989) for multiple resources.

Hence, we can apply for the LSP-BD- $\mathrm{R}_{2, \rho<1}$ with a possible ordering amount throughout the periods $1,2, \ldots, j$ the method proposed by Beasley and Christofides (1989), which is based on 
Lagrangian relaxation. The authors use the subgradient optimization which approximately solves the Lagrangian dual to get lower and upper bounds for the optimal solution of this problem and resolve it using a tree search procedure. Finally, the optimal cost of the general LSP-BD- $R_{2, \rho<1}$ is the minimum cost over all the possible values of ordering amount throughout the periods $1,2, \ldots, j$.

\subsection{LSP-BD-R with return possibility only in procurement peri- ods (LSP-BD-R ${ }_{3}$ )}

This section focuses on solving the LSP-BD-R with another type of the buyback contract in which the return periods must coincide with procurement periods. This type of contract mainly concerns the products with a transportation cost from the retailer to the supplier being higher than the return revenue. Then, the retailer signs the contract $R_{3}$ in order to avoid taking over the freight of returned quantities.

In every period $t$ of the planning horizon $1 . . T$, the retailer can return a quantity $q_{t}$ lower than the ordered quantity $x_{t}$. If $x_{t}=0$, then the return in period $t$ is prohibited, otherwise, it is allowed. Besides, the total returned quantity across the horizon is restricted to $\rho \sum_{t=1}^{T} x_{t}$ units. The general assumption we made for this contract is $\max _{t=1, \ldots, T}\left\{p_{t}^{b}\right\}<p_{t}, \forall t \in\{1, \ldots, T\}$.

The mathematical formulations with or without inventory variables of LSP-BD- $R_{3}$ are the same ones as the LSP-BD-R $\mathrm{R}_{2}$ for $j=T$, but, with the additional constraint $q_{t} \leq x_{t}, \forall t \in$ $\{1, \ldots, T\}$. The question that we address is the following: Given that a retailer seeks to minimize his costs when ordering an item, when and how much to order, to return and to dispose throughout the horizon? We will study first the case with $\rho=1$ and second with $\rho<1$.

\subsubsection{LSP-BD-R , $_{3}$ with full return policy (LSP-BD- $\mathbf{R}_{3, \rho=1}$ )}

In the same way as before, we will decompose the LSP-BD- $R_{3, \rho=1}$ into subplans which start and end with zero stock. We will determine the minimum costs $\mathscr{C}(u, v)$ of all subplans $(u, v)$ for $1 \leq u<v \leq T+1$ in which we have to satisfy the demands $d_{u}, \ldots, d_{v-1}$, return in procurement periods and dispose the remaining quantity after the buyback. After that, we can compute the total optimal cost $C_{T}$ of the LSP-BD- $R_{3, \rho=1}$ by using the same shortest path algorithm (3.1) used in Section 3.3.1. Using Example 3.5, we compare the optimal schedules of LSP-BD- $R_{3, \rho=1}$ and LSP-BD-R $2, \rho=1$ for $j=T$.

Example 3.5. The same data of Example 3.4 is used, but modifying the return revenues as $p_{1}^{b}=2, p_{2}^{b}=10$ and $p_{3}^{b}=13$. Table 3.5 shows that the total cost of LSP-BD-R $\mathrm{R}_{2, \rho=1}$ for $j=3$ is less than the one of LSP-BD- $\mathrm{R}_{3, \rho=1}$, because the latter has more restrictions on return periods. In addition, we note that, in LSP-BD- $\mathrm{R}_{3, \rho=1}$, the optimal return period of the subplan $(1,4)$ does not coincide with period 2 having $\max _{i=1, \ldots, 3}\left\{\tilde{p}_{i}^{b}\right\}=22$, since period 2 is not a procurement period.

We will develop an algorithm of $O\left(T^{5}\right)$ time complexity for LSP-BD-R $\mathrm{R}_{3, \rho=1}$ by using the following optimality properties.

Some preliminaries and properties:

We use Property 3.10 which affirms that there is at most one return period and no disposal in a subplan $(u, v)$, and, the returned quantity is equal to its remaining units $\left(R_{u, v-1}\right)$. The proof of this property is composed of two parts. We use the first part to prove that there is at most one return in a subplan. In order to prove that there is no disposal, we can refer to the second part 
Table 3.5: The optimal quantities of LSP-BD-R $\mathrm{R}_{2, \rho=1}$ for $j=3$ and LSP-BD-R $\mathrm{R}_{3, \rho=1}, V=4$

\begin{tabular}{c|c|cccc||cccc}
$V=4$ & \multicolumn{1}{c}{ LSP-BD-R ${ }_{2, \rho=1} j=3$} & \multicolumn{4}{c}{ LSP-BD-R $_{3, \rho=1}$} \\
\hline Period $t$ & $p_{t}^{b}$ & $x_{t}$ & $s_{t}$ & $q_{t}$ & $e_{t}$ & $x_{t}$ & $s_{t}$ & $q_{t}$ & $e_{t}$ \\
\hline 1 & 20 & 4 & 3 & 0 & 0 & 4 & 2 & 1 & 0 \\
2 & 22 & 0 & 0 & 2 & 0 & 0 & 1 & 0 & 0 \\
3 & 19 & 4 & 0 & 3 & 0 & 0 & 0 & 0 & 0 \\
\hline Costs & & \multicolumn{4}{c|}{65} & & \multicolumn{5}{c}{69} &
\end{tabular}

of the proof if the period $t$ in which we have $e_{t}>0$ is an ordering period. Otherwise, we choose the ordering period $i$ just before period $t(i<t)$, decrease $e_{t}$ by $e_{t}, s_{k}$ by $e_{t}$ for $k=i, \ldots, t-1$ (we have $s_{i} \geq s_{i+1} \geq \cdots \geq s_{t-1} \geq e_{t}$ because $x_{i+1}=x_{i+2}=\cdots=x_{t}=0$ ), and increase $q_{i}$ by $e_{t}$. Thus, we obtain a new feasible solution with a lower cost, decreasing by an amount of $\left(\tilde{p_{i}^{b}}-\tilde{p_{t}^{e}}\right) e_{t}$. We have $\tilde{p_{i}^{b}} \geq \tilde{p_{i}^{e}}$ and $\tilde{p_{i}^{e}} \geq \tilde{p_{t}^{e}}$ because $i<t$.

Property 3.13 remains valid for this problem whereby we can compute the entering inventory level value in each ordering period.

Computation of $\mathscr{C}(u, v)$ :

For a given subplan $(u, v)$, we can compute the quantity returned but we do not know the position of the return period. We have to find the optimal return period. Hence, we suppose that the return period can be the period $t$ such that $t \in\{u, \ldots, v-1\}$. The optimal cost of $(u, v)$ is as follows:

$$
\mathscr{C}(u, v)=\min _{t \in\{u, \ldots, v-1\}}\left\{\mathscr{C}_{v, t}^{(4)}(u, v)\right\}
$$

knowing that the cost $\mathscr{C}_{v, t}^{(4)}(u, l+1)$ is the minimum cost to satisfy $D_{u, l}$ with period $t$ being both a period of return and period of ordering in $(u, v)$, and period $l+1$ being a replenishment period with $1 \leq u \leq l \leq v-1 \leq T$.

For $l=u, \ldots, t-1$, the dynamic programming formulation of $\mathscr{C}_{v, t}^{(4)}(u, l+1)$ is the same as $\mathscr{C}_{v}^{(3)}(u, l+1)$ and we obtain $\mathscr{C}_{v, t}^{(4)}(u, t)=\mathscr{C}_{v}^{(3)}(u, t)$. To ensure that period $t$ is an ordering period, we compute the minimum cost $\mathscr{C}_{v, t}^{(5)}(u, l+1)$ for $t \leq l \leq v-1$ in which we meet the demands of periods $u, \ldots, l$ and we make sure that the periods $t$ and $l+1$ are two consecutive ordering periods. Then, we have:

$$
\mathscr{C}_{v, t}^{(5)}(u, l+1)=\mathscr{C}_{v, t}^{(4)}(u, t)+f_{t}+\left(p_{t}+\frac{a_{t}}{V}\right)\left(s_{l}+D_{t, l}+q_{t}^{u, v}-s_{t-1}\right)+H_{u, v}^{(3)}(t, l+1)-p_{t}^{b} q_{t}^{u, v}
$$

The values of inventory levels $s_{l}$ and $s_{t-1}$ are determined by Property 3.13 according to the position of the return period $t$. Moreover, the values of inventory cost $H_{u, v}^{(3)}(t, l+1)$ are calculated in Section 3.4.1.

For $l=t+1, \ldots, v-1$, we formulate the $\operatorname{costs} \mathscr{C}_{v, t}^{(4)}(u, l+1)$ as an efficient dynamic program:

Recurrence relation. For $l=t+1, \ldots, v-1$,

$$
\begin{gathered}
\mathscr{C}_{v, t}^{(4)}(u, l+1)=\min \left\{\min _{t \leq k<l}\left\{\mathscr{C}_{v, t}^{(4)}(u, k+1)+f_{k+1}+\left(p_{k+1}+\frac{a_{k+1}}{V}\right)\left(s_{l}+D_{k+1, l}-s_{k}\right)+H_{u, v}^{(3)}(k+1, l+1)\right\} ;\right. \\
\left.\mathscr{C}_{v, t}^{(5)}(u, l+1)\right\}
\end{gathered}
$$


Boundary condition. $\mathscr{C}_{v, t}^{(4)}(u, t+1)=\mathscr{C}_{v, t}^{(5)}(u, t+1)$

Objective. $\mathscr{C}_{v, t}^{(4)}(u, v)$

In order to obtain the $\operatorname{cost} \mathscr{C}_{v, t}^{(4)}(u, l+1)$, for $l=t+1, \ldots, v-1$, we suppose that there is either an ordering period $k+1$ such that there is no acquisition taking place in periods $k+2, k+3, \ldots, l$, where $t<k+1<l+1$, or, there is an ordering period $t$ such that the periods $t$ and $l+1$ are two consecutive ordering periods. The values of these costs can be obtained recursively in $O\left(T^{4}\right)$ time. Therefore, the overall complexity of the computation of the cost $\mathscr{C}(u, v)$ is $O\left(T^{5}\right)$.

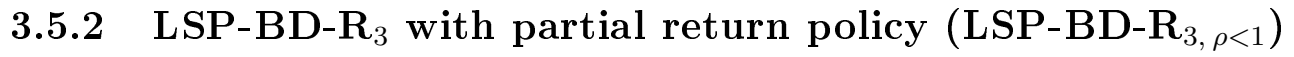

In this section, we add the fact that the total quantity returned over the planning horizon $(1 . . T)$ is limited by $\rho \sum_{t=1}^{T} x_{t}$ knowing that the returns are only allowed in procurement periods. We will prove that this problem is also similar to RCSPP. After that, we will add a comment in which we prove that the LSP-BD- $\mathrm{R}_{3, \rho<1}$ and the LSP-BD-R $\mathrm{R}_{2, \rho<1}$ with decreasing modified return revenues are equivalent.

The LSP-BD-R $3, \rho<1$ verifies Property 3.7 for $k=T$ which is equivalent to Property 3.17 for $j=T$ because we have a null stock level in period $T, s_{T}=0$. These two equivalent properties show that the quantity purchased throughout the planning horizon is bounded on both sides as follows: $\sum_{t=1}^{T} x_{t}=\left(\left\lceil\frac{D_{1, T}}{V}\right\rceil+\Delta\right) V$ with $0 \leq \Delta \leq T-1$.

The graph of the LSP-BD- $R_{3, \rho<1}$ is built in the same way as the graph of the general LSP$\mathrm{BD}-\mathrm{R}_{2, \rho<1}$. The only differences are the values of the optimal cost $\mathscr{F}_{\phi}^{(v)}(u, v)$ and the optimal total quantity purchased $\psi_{\phi}(u, v)=\sum_{i=u}^{v-1} x_{i(u, v)_{\phi}}=\left\lceil\frac{D_{u, v-1}}{V}\right\rceil V$ in each arc $(u, v)_{\phi}$, being the subplan $(u, v)$ with $\phi$ units of returned quantity. Now the question that we have to ask is: how to calculate the cost $\mathscr{F}_{\phi}^{(v)}(u, v)$ for the problem with the buyback contract $\mathrm{R}_{3, \rho<1}$ ? To answer this question, we proceed in the same way as the computation of the cost $\mathscr{C}(u, v)$ in Subsection 3.5.1 $(\rho=1)$. In the case with $\rho=1$, all the remaining quantity in the subplan $(u, v)$ is returned to the supplier. However, in the case with $\rho<1$, the optimal returned quantity of each subplan $(u, v)$ depends on the ones of other subplans. The optimal cost of the $\operatorname{arc}(u, v)_{\phi}$ is as follows:

$$
\mathscr{F}_{\phi}^{(v)}(u, v)=\min _{t \in\{u, \ldots, v-1\}}\left\{\mathscr{C}_{v, t}^{(4)}(u, v)_{\phi}\right\}
$$

The cost $\mathscr{C}_{v, t}^{(4)}(u, l+1)_{\phi}$ is the minimum cost to satisfy $D_{u, l}$ with period $t$ being a period of ordering and return in the subplan $(u, v)$ with $\phi$ units of return, and period $l+1$ being a replenishment period with $1 \leq u \leq l \leq v-1 \leq T$. For this cost, there are $\left\lceil\frac{D_{u, v-1}}{V}\right\rceil V-D_{u, v-1}-\phi$ units of disposal.

For $l=u, \ldots, t-1$, the dynamic programming formulation of $\mathscr{C}_{v, t}^{(4)}(u, l+1)_{\phi}$ is the same one of $\mathscr{C}_{v}^{(1)}(u, l+1)$ with $e_{u}^{u, v}=\left\lceil\frac{D_{u, v-1}}{V}\right\rceil V-D_{u, v-1}-\phi\left(\mathscr{C}_{v}^{(1)}(u, t)_{\phi}\right)$ and we obtain $\mathscr{C}_{v, t}^{(4)}(u, t)_{\phi}=$ $\mathscr{C}_{v}^{(1)}(u, t)_{\phi}$. For $l=t, \ldots, v-1$, refer to Subsection 3.5.1 to compute $\mathscr{C}_{v, t}^{(4)}(u, l+1)_{\phi}$ by changing the value of $q_{t}^{u, v}$ by $\phi$.

Now, we can transform the graph $G$ of LSP-BD-R $\mathrm{R}_{3, \rho<1}$ into the graph $G^{\prime}$ having only one arc between each pair of nodes. Then, for each possible value of $\sum_{i=1}^{T} x_{i}$, which means for $\Delta$, the LSP-BD-R ${ }_{3, \rho<1}$ can be formulated as a RCSPP whose objective is to find the minimum cost of a path from node 1 to the last node of the transformed network $G^{\prime}$ such that the total quantity purchased in this path is equal to $\left(\left\lceil\frac{D_{1, T}}{V}\right\rceil+\Delta\right) V$ and the total return amount along the path is at most $\rho\left(\left\lceil\frac{D_{1, T}}{V}\right\rceil+\Delta\right) V$. We apply the method of Beasley and Christofides (1989) to solve it as 
the LSP-BD- $\mathrm{R}_{2, \rho<1}$ with $M$ ordering amount throughout the periods $1,2, \ldots, j$. The optimal cost of the LSP-BD-R $R_{3, \rho<1}$ is the minimum cost of the LSP-BD-R $\mathrm{R}_{3, \rho<1}$ with $\left(\left\lceil\frac{D_{1, T}}{V}\right\rceil+\Delta\right) V$ total order units over all the possible values of $\Delta$.

\section{Comment}

We consider LSP-BD-R $\mathrm{R}_{3, \rho<1}$ with decreasing modified return revenues $\left(\tilde{p_{1}^{b}}<\tilde{p_{2}^{b}}<\cdots<\tilde{p}_{T}^{\tilde{b}}\right)$ which helps to easily identify the return period in each possible subplan $(u, v)$. We will show that this special case is equivalent to the LSP-BD- $\mathrm{R}_{2, \rho<1}$ for $j=T$.

Now, we prove the equivalence between the LSP-BD-R $\mathrm{R}_{3, \rho<1}$ and the LSP-BD-R $\mathrm{R}_{2, \rho<1}$ for $j=T$, by considering decreasing modified return revenues. We have to ensure that both problems have the same return and disposal periods in a subplan $(u, v)$. The LSP-BD- $\mathrm{R}_{2, \rho<1}$ with $j=T$ and decreasing modified return revenues verifies Property 3.14 in which for any subplan $(u, v)$, there is at most one disposal in $u$ and one return in $u$ because $\tilde{p_{u}^{b}}=\max _{i=u, \ldots, v-1}\left\{\tilde{p_{i}^{b}}\right\}$ and period $u$ is the only period having the maximum of modified return revenues. Therefore, we obtain $e_{u}^{u, v}+q_{u}^{u, v}=\left\lceil\frac{D_{u, v-1}}{V}\right\rceil V-D_{u, v-1}$.

The LSP-BD-R $\mathrm{R}_{3, \rho<1}$ with decreasing modified return revenues also verifies Property 3.14 . In order to prove the existence of at most one disposal and one return in a given subplan $(u, v)$, we follow the same proof of Property 3.14. In addition, for this special case, if there is a disposal, then it must be in period $u$, which is proved in the second part of the proof of Property 3.1. Furthermore, the optimal return period is placed in period $u$ by also applying the second part of the proof of Property 3.1 in which we make the following modification: we consider an optimal policy $\pi^{\prime}$ in which there is a subplan $(u, v)$ with $q_{j}>0$ and $j>u$. Thus, the quantity $\alpha_{i, j}$ depends on the quantity returned and not on the quantity disposed of, so $\alpha_{i, j}^{\prime}=\min \left\{q_{j}, \min _{k=i, \ldots, j-1} s_{k}\right\}>0$. We carry out the same modification performed in the proof of Property 3.1 to obtain a new policy $\pi^{*}$ having either one subplan $(u, v)$ with $u$ being the return period, or, a division of $(u, v)$ into several subplans whose return periods are placed in the beginning. The total cost of $\pi^{*}$ is computed as a function of the total cost of $\pi^{\prime}$, as follows:

$$
\mathscr{C}\left(\pi^{*}\right)=\mathscr{C}\left(\pi^{\prime}\right)-\alpha_{i, j}^{\prime}\left(\tilde{p_{u}^{b}}-\tilde{p_{j}^{b}}\right)
$$

Since the modified return revenues are decreasing, then we obtain $\mathscr{C}\left(\pi^{*}\right)<\mathscr{C}\left(\pi^{\prime}\right)$ which means that the solution of the policy $\pi^{\prime}$ cannot be optimal - a contradiction.

We also note that the special case of this section verifies Property 3.15 in which we can determine the values of the entering inventory levels in each ordering period $k$ of $\operatorname{subplan}(u, v)$ having one disposal in $u$ and one return in $u$.

In a given subplan, the only difference between the LSP-BD- $R_{2}$ and LSP-BD- $\mathrm{R}_{3}$ is the position and the nature of the return period. By assuming the constraint of decreasing modified return revenues in the two problems, we get the same return periods in each possible subplan. Hence, these two problems are equivalent.

\subsection{Computational experiments}

In this section, for each problem solved by a polynomial time dynamic programming algorithm (DP), we compare the resolution time of its MILP and its DP under different parameters. The tests are performed with the same procedure as those of $\mathrm{LSP}_{-\mathrm{BR}_{1}}$ with $w \geq 1$ (see Section 2.6): execution on an Intel Core $2.40 \mathrm{GHz}, 8 \mathrm{~GB}$ RAM, implementation of the MILP in Xpress Mosel 
version 3.10, and the DP in Java, the use of aggregate formulations, and the generation of 10 instances to obtain the average for each configuration.

We proposed a DP with polynomial time complexity for each of the four following problems: LSP-BD-R $_{1, \rho=1}$, LSP-BD-R $1, \rho<1$, LSP-BD-R $2, \rho=1$, and LSP-BD-R ${ }_{3, \rho=1}$. The common parameters that affect the resolution time of their MILP are: length of the horizon, demand, batch size, and cost and revenue parameters for which we consider the same configurations as in Chapter 2 : - Length of the horizon: $T=20, T=50$ and $T=100$,

- Demand and batch sizes: $D 1=U(5,20)$ with $V 1=4$ and $D 2=U(30,100)$ with $V 2=10$,

- Setup costs: $f_{t}=U(50,100)$,

- Unit procurement costs: $p_{t}=0.01 f_{t}$,

- Fixed costs per batch replenished: $a_{t}=0.1 f_{t}$,

- Inventory holding costs: $h_{t}=U(0.05,2)$,

- One unit return revenue: $p_{w}^{b}=0.4 \max _{t=1, \ldots, T}\left\{p_{t}\right\}$ for LSP-BD-R $\mathrm{R}_{1, \rho=1}$ and LSP-BD-R $1, \rho<1$, and unit return revenues: $p_{t}^{b}=0.4 p_{t}$ for LSP-BD- $\mathrm{R}_{2, \rho=1}$ and LSP-BD-R $\mathrm{L}_{3, \rho=1}$.

In the MILP of LSP-BD- $R_{1, \rho=1}$, we have one supplementary parameter which is the periodicity of the return periods which is considered equal to $T$. For LSP-BD-R L $_{1, \rho<1}$, we have two additional parameters: the periodicity of the return periods, $w_{1}=T$, and the maximum return percentage that we consider $\rho=0.02$. For LSP-BD-R $\mathrm{R}_{2, \rho=1}$, we have one more parameter: a time limit on returns with $j=15$.

In Table 3.6, we provide the performance of the proposed methods for the four problems. The optimal solutions of LSP-BD-R ${ }_{1, \rho=1}$, LSP-BD-R $1, \rho<1$, LSP-BD- $_{2, \rho=1}$ and LSP-BD-R L $_{3=1}$, in all tested instances $T=20$ and $T=50$, are obtained quite instantaneously whether by MILP or by DP. For the instances with $T=100$, the execution time of DP is greater than the one of MILP. We note for example, that the time of DP proposed for LSP-BD-R R $_{3=1}$ is about 1 minute, whereas the one of MILP is about 3 seconds.

Table 3.6: Computational results for DP and MILP of the problems with disposal concept

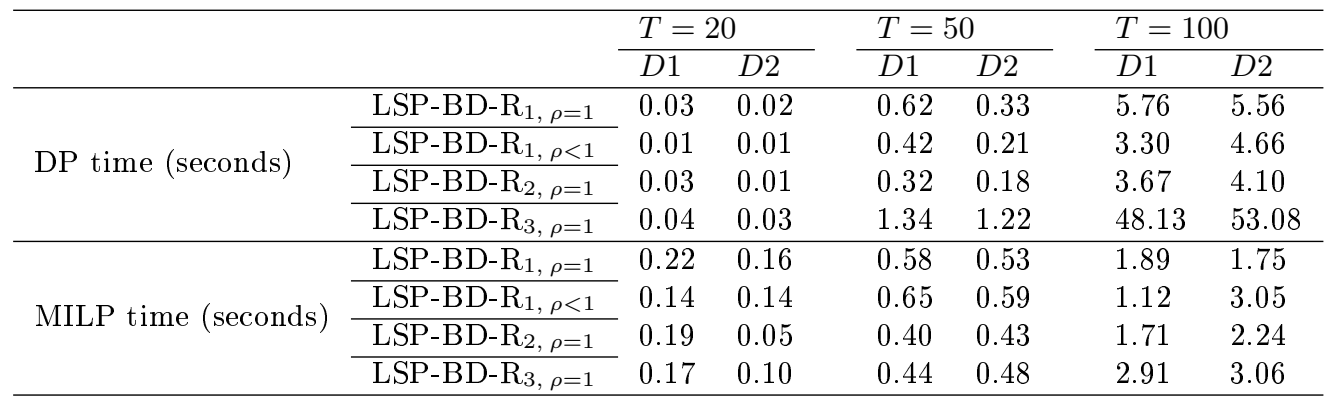

\subsection{Conclusion}

In this chapter, we studied the single-item uncapacitated LSP under a buyback contract with batch ordering and disposal concept (LSP-BD-R). We considered three types of buyback contract: the type $R_{1}$ with fixed return periods, the type $R_{2}$ with a time limit for returns and the type $R_{3}$ with returns only in procurement periods. These problems are addressed either with full return $(\rho=1)$, or, with partial return $(\rho<1)$. We present in Figure 3.7 the different cases studied in this chapter with the complexity results. 


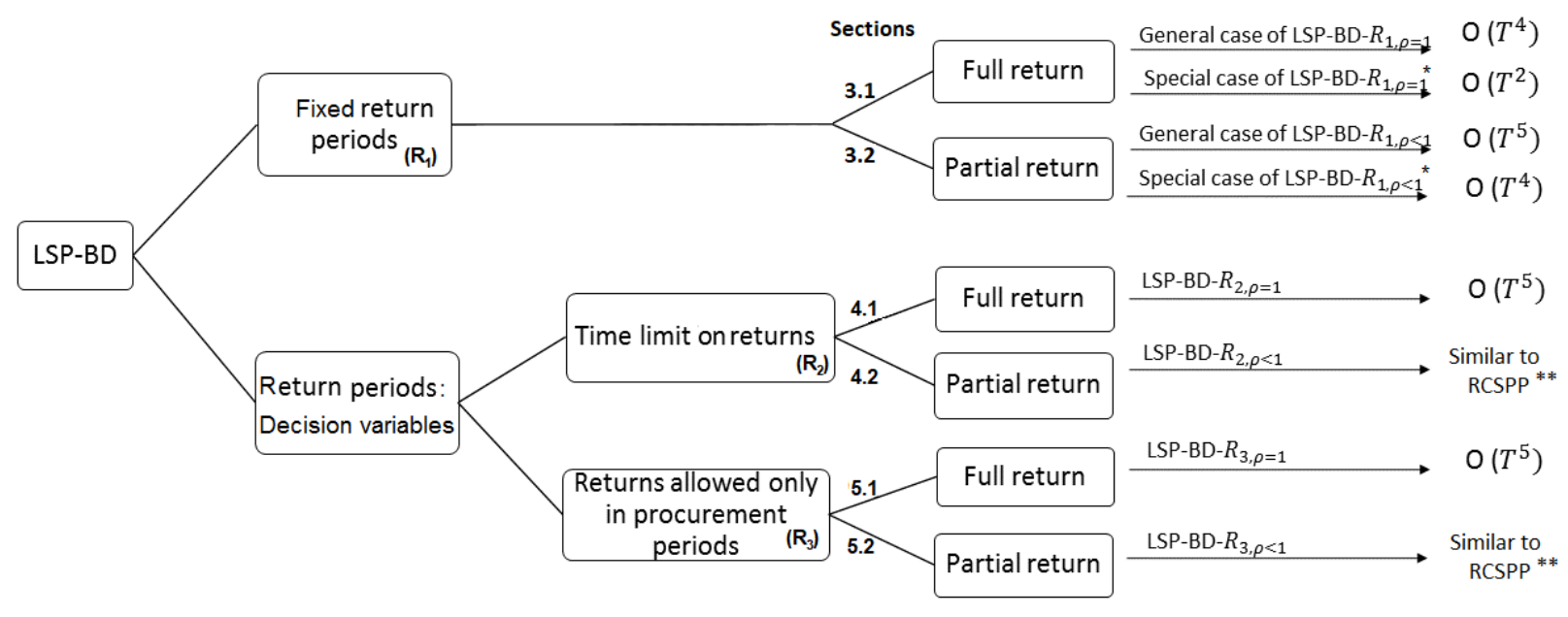

Figure 3.7: Different cases studied in Chapter 3

(*: additional assumption on the trade-off between return revenue and holding cost)

(**: Resource Constrained Shortest Path Problem)

In our problems, we assume null disposal costs. However, the LSP-BD-R with non-negative and non-positive disposal costs are not difficult to solve. We have just to make some modifications on the proposed algorithms. In these problems we have to ensure this following assumption: $\max _{i=1, \ldots, T}\left\{p_{i}^{e}\right\}<p_{t}, \forall t \in\{1, \ldots, T\}$ in order to avoid the speculation. The resolution method for each buyback form $\mathrm{R}_{i}, \forall i=1,2,3$ looks like the ones proposed for our problem. We have to consider in each subplan $(u, v)$ the values of $\tilde{p}_{t}^{e}=p_{t}^{e}+\sum_{k=t}^{T} h_{k}$ with $p_{t}^{e} \in \mathbb{R}, \forall t=u, \ldots, v-1$ and to compare $\max _{i=u, \ldots, v-1}\left\{\tilde{p}_{i}^{b}\right\}$ and $\max _{i=u, \ldots, v-1}\left\{\tilde{p}_{i}^{e}\right\}$, for $1 \leq u<v \leq T+1$ in order to decide if the return or the disposal is more profitable for the retailer. Therefore, we can say that the buyback contracts considered in this study can represent disposal concepts with non-negative disposal revenues and disposal constraints depending on the value of the maximum return $\rho$.

In the next Chapter, we model first the LSP-B with capacity reservation contract. Second, we define and give the mathematical formulations of the different types of CRC integrated with the single-item LSP. 
CHAPTER 3. FULL BATCH REPLENISHMENT WITH BUYBACK AND DISPOSAL 


\section{Chapter 4}

\section{Modeling lot sizing problems with batch ordering (LSP-B) under different capacity reservation contracts (CRC)}

We consider in this chapter that there is a capacity reservation contract signed between two members of a supply chain who need to plan the batch procurement activity. In this LSP with batch ordering (LSP-B), the products are ordered in batches with FTL cost structure. We first model this LSP under the general capacity reservation contract (LSP-B-CRC) and we address different scenarios that the company can have: the reserved capacity being either fixed or decision variable (stationary or time-dependent).

There are other contractual forms for representing the capacity reservation: deductible reservation, take-or-pay, pay-to-delay, quantity flexibility, backup agreement, minimum commitment, and revenue-sharing contracts. Second, we integrate all these contracts into the multi-period lot sizing problem with batch ordering by proposing mixed integer linear programs. 


\subsection{Introduction}

In the capacity reservation contract (CRC) considered in this chapter, the supplier and the retailer decide, in each period $t$, a certain level of capacity $R_{t}$ to reserve at the supplier over $T$ periods, where $R_{t}$ is the number of batches reserved at a cost $r_{t}$. In return, the supplier provides the retailer a number of batches being less than $R_{t}$ for a lower fixed cost $a_{t}$ (regular cost) per batch replenished. The supplier also allows the retailer to procure more batches exceeding the initial capacity $R_{t}$, but at a higher cost $b_{t}$ (particular cost). The supplier ensures that, in each period $t$, the costs of reservation and regular procurement of one batch is less than the particular procurement cost, $r_{t}+a_{t} \leq b_{t}$. In addition to the costs related to batches, the CRC may incur other costs: a setup cost $f_{t}$ paid for any positive amount ordered per period, and a unit procurement cost $p_{t}$ for each unit ordered per period. This contract provides a risk-sharing mechanism and offers the buyer the flexibility to deal with uncertain demand and guarantee a capacity before receiving orders; and provides a security to the supplier.

The CRC has several variations existing in the literature. In this work, we consider, on the one hand, different assumptions on the reserved capacity (time-dependent decision variable, or constant decision variable, or fixed) and on the other hand, numerous forms being equivalent to CRC. Park and Kim (2014) state that capacity reservation contracts proposed in previous studies can be divided into two groups: general contracts that are used in retail and manufacturing industries, and specialized contracts which are frequently used in capital-intensive industries. General contracts include: quantity flexibility, backup agreement, minimum commitment, revenue-sharing and buyback contracts. The latter is addressed in Chapters 2 and 3. And specialized contracts include: deductible reservation, take-or-pay and pay-to-delay contracts. In the literature, the main focus of published studies dealing with these different contracts, as with the newsvendor problem, has been on single-period models in the stochastic case taking into account only the unit price. This chapter deals with mathematical models for those contracts integrated into the LSP with batch ordering, so we add the fixed cost per batch.

In Section 4.2, we elaborate a general model of LSP-B-CRC with appropriate algorithms for different assumptions on capacity. The goal is to determine the capacity to reserve in batches if it is a decision variable and the optimal quantities to procure by minimizing the total cost. In Section 4.3, we discuss mathematical models that solve LSP under specialized contracts and thereafter under general contracts, for the deterministic, multi-period, single-item, and singlecontract case taking into account batch ordering. Section 4.4 summarizes this chapter.

\subsection{Modeling the LSP-B-CRC}

We consider that a retailer signs a capacity reservation contract over a horizon of $T$ periods with a supplier to reserve for each period $t$ a capacity $R_{t}$ expressed in number of batches at a reservation price $r_{t}$. At the beginning of each period $t$, the retailer orders a quantity $x_{t}$ at a unit price $p_{t}$ in order to satisfy its deterministic demand $d_{t}$. If the desired quantity is ordered in period $t$, then a binary setup variable $y_{t}$ receives the value of 1 and a setup cost $f_{t}$ is incurred, otherwise it is zero.

The supplier delivers the purchased quantity in batches of size $V_{t}$. When the number of ordered batches is smaller than $R_{t}$, denoted by $A_{t}$, then the retailer pays a cost $a_{t}$ per batch (which does not contain the unit cost $p_{t}$ ). If he requires more than this capacity $R_{t}$ to meet its additional demand, then he procures the excess batches, denoted by $B_{t}$ at a price $b_{t}$ with $r_{t}+a_{t} \leq b_{t}$. Otherwise $\left(r_{t}+a_{t}>b_{t}\right)$, the retailer does not need to sign such a contract with the 
supplier, because it is not profitable for him, then he will resort for example to the spot market.

At the end of period $t$, a quantity $s_{t}$ is stored incurring a unit inventory cost $h_{t}$. We assume, without loss of generality, that the stock at the beginning of the planning horizon is null.

There are two different instants of decisions. In the first instant, the retailer and the supplier determine the optimal values of capacity to reserve in each period over the horizon, and in the second instant, they determine the optimal values of quantity to procure in batches from supplier. Traditionally, in several companies, capacity reservation, procurement and inventory decisions are decided separately in a hierarchical process. However, Bradley and Arntzen (1999) affirm that simultaneous consideration of capacity, procurement, and inventory decisions maximizes the financial results, because, it leads to balance the trade-off between having insufficient capacity in some periods and excess capacity in others. Thus, we give a general model of the LSP-B-CRC in which the reserved capacity is a decision variable in addition to the procurement and inventory decision variables. In the second instant of decision, the reserved capacity becomes fixed in the model.

In Table 4.1, we find all the notations that are used in the general model of LSP-B-CRC.

Table 4.1: Notations for the general model of the LSP-B-CRC

\begin{tabular}{|c|c|}
\hline \multicolumn{2}{|c|}{ Parameters } \\
\hline$T$ & length of the horizon \\
\hline$p_{t}$ & unit procurement cost in period $t$ \\
\hline$f_{t}$ & setup cost in period $t$ \\
\hline$r_{t}$ & reservation cost per batch in period $t$ \\
\hline$a_{t}$ & $\begin{array}{l}\text { fixed cost per batch replenished under the reserved capacity in } \\
\text { period } t\end{array}$ \\
\hline$b_{t}$ & $\begin{array}{l}\text { fixed cost per batch replenished exceeding the reserved capacity } \\
\text { in period } t\end{array}$ \\
\hline$h_{t}$ & inventory holding cost per unit at the end of period $t$ \\
\hline$d_{t}$ & demand in period $t$ \\
\hline$V_{t}$ & batch size in period $t$ \\
\hline$R_{t}$ & number of batches reserved for period $t$ ( 2 nd decision instant) \\
\hline \multicolumn{2}{|c|}{ Decision variables } \\
\hline$x_{t}$ & amount of procurement in period $t$ \\
\hline & $\int 1$ if a procurement takes place in period $t$ \\
\hline $\mathrm{y}_{t}$ & 0 otherwise \\
\hline$R_{t}$ & number of batches reserved for period $t$ (1st decision instant) \\
\hline$A_{t}$ & number of batches ordered with the cost $a_{t}$ in period $t$ \\
\hline$B_{t}$ & number of batches ordered with the cost $b_{t}$ in period $t$ \\
\hline$s_{t}$ & stock level at the end of period $t$ \\
\hline
\end{tabular}

The aim of the general model of LSP-B-CRC is to appropriately decide the number of batches $R_{t}$ to reserve in each period $t$ at the first decision instant, and $A_{t}$ and $B_{t}$ to replenish in each period $t$ at the second decision instant, respecting the capacity limits and batch ordering, meeting the demands without backlogging, and minimizing the total cost. The two decision levels of CRC are illustrated in Figure 4.4 .

We present the aggregate formulation of LSP-B-CRC as MILP as following: 

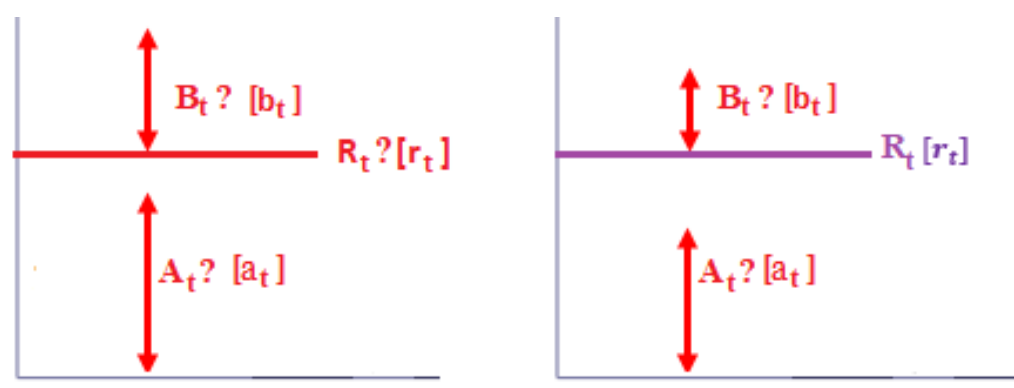

Figure 4.1: Illustration of the principle of $\mathrm{CRC}$ at a given period $t$ at the 1 st and 2 nd decision levels

$$
\begin{array}{lll}
\min & \sum_{t=1}^{T}\left(r_{t} R_{t}+f_{t} y_{t}+p_{t} x_{t}+a_{t} A_{t}+b_{t} B_{t}+h_{t} s_{t}\right) & \\
\text { s.t. } & x_{t}+s_{t-1}=d_{t}+s_{t} & \forall t=1, \ldots, T \\
& x_{t} \leq\left\lceil\frac{\sum_{j=t}^{T} d_{j}}{V_{t}}\right\rceil V_{t} y_{t} & \forall t=1, \ldots, T \\
& x_{t} \leq\left(A_{t}+B_{t}\right) V_{t} & \forall t=1, \ldots, T \\
& 0 \leq A_{t} \leq R_{t} & \forall t=1, \ldots, T \\
& s_{0}=0 & \\
& x_{t}, s_{t} \in \mathbb{R}_{+} & \forall t=1, \ldots, T \\
& A_{t}, B_{t}, R_{t}, \in \mathbb{N} & \forall t=1, \ldots, T \\
& y_{t} \in\{0,1\} & \forall t=1, \ldots, T
\end{array}
$$

The objective function (4.1) is to minimize the costs of capacity reservation, setup, procurement (unit and batch) and inventory over the planning periods. Constraints (4.2) represent the satisfaction of the demand at period $t$ by the procurement of period $t$ and/or from the stock of period $t-1$. The remainder is put in the stock at the end of period $t$. Constraints (4.3) link the quantity to be ordered with the binary variable of setup. Constraints (4.4) ensure that the number of batches replenished $\left(A_{t}+B_{t}\right)$ is sufficient for the quantity ordered $x_{t}$ at each period $t$. Constraints (4.5) ensure that the number of batches replenished at a cost $a_{t}$ must be less than the number of reserved batches. Constraint (4.6) sets the stock at the beginning of the planning horizon to zero. Constraints (4.7) to (4.9) are sign constraints. In instant $1, R_{t}$ is a decision variable, but no more in instant 2 . The same model is used for both decision instants.

In the first instant of decision in LSP-B-CRC, the reserved capacity $R_{t}$ is a decision variable which is computed by using the first demand forecasts. Therefore, this problem is at tactical level. In the second instant of decision in LSP-B-CRC, the reserved capacity $R_{t}$ is fixed and the retailer places orders by using the revised demand forecasts. This problem is at operational level. Now, we discuss the different cases of LSP-B-CRC in this sequence: fixed (studied in literature), stationary decision variable, time-dependent decision variable reserved capacities. We show that the resolution method proposed for the operational LSP-B-CRC (fixed $R_{t}$ ) can be adapted to solve the tactical LSP-B-CRC (decision variable $R_{t}$ ). 


\subsubsection{LSP-B-CRC with fixed reserved capacity}

In this problem, we assume that $R_{t}$ is known (decision instant 2). Thus, $\sum_{t=1}^{T} r_{t} R_{t}$ becomes a constant, this one does not impact the optimization, then we will delete it from the objective function (4.1). In Figure 4.2 (see periods 1 and 7), we show that, in CRC with fixed reserved capacity, the retailer may procure extra batches $B_{t}$ only if the regular batches $A_{t}$ are already at full reserved capacity $R_{t}$. In period 3 , the retailer purchases all the reserved batches $R_{3}$ with a unit cost $a_{3}$. We can also have periods in which the value of $A_{t}$ is less than the reserved capacity $R_{t}$ (see period 5).

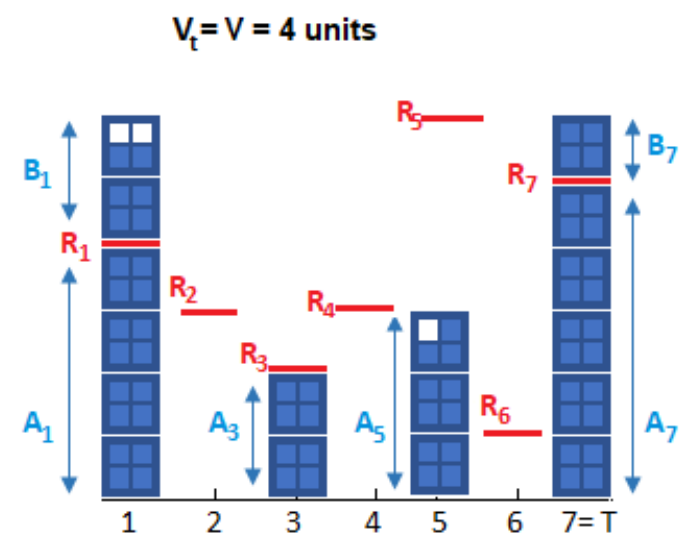

Figure 4.2: Possible values of $A_{t}$ and $B_{t}$ in LSP-B-CRC with fixed reserved capacity, a stationary batch size $V$ and $T=7$

The LSP-B-CRC with fixed reserved capacity is studied by Akbalik et al. (2017). The authors affirm that this problem is NP-hard in the ordinary sense. They adapt the pseudo-polynomial time dynamic programming algorithm proposed by Florian et al. (1980) to solve the LSP-B-CRC with fixed $R_{t}$. The complexity of the recursive formulation is $O\left(T\left(D_{1, T}\right)^{2}\right)$ time with $D_{1, T}$ being the cumulative demand from period 1 to period $T$. They also classify different NP-hard cases and propose polynomial time algorithms to solve several special cases of this problem.

\subsubsection{LSP-B-CRC with stationary reserved capacity being a decision variable}

In this problem, $R_{t}=R$ is a stationary decision variable (decision instant 1 ). The LSP-B-CRC with stationary reserved capacity being a decision variable is NP-hard due to the time-varying batch sizes and cost parameters considered. In Akbalik and Rapine (2013), the authors show that if any one of the cost parameters is allowed to be time-dependent in the uncapacitated LSP-B with time-dependent batch sizes, the problem becomes NP-hard. To solve this problem, we either use the MILP, or, we compile for each possible value of $R$ the pseudo-polynomial time dynamic programming algorithm proposed by Akbalik et al. (2017) and then we find the value $R$ minimizing the total cost. First, we use the formulation without inventory variables to model the LSP-B-CRC with variable $R$, by replacing in the first formulation, the inventory variable $s_{t}$ with $\sum_{i=1}^{t}\left(x_{i}-d_{i}\right), \forall t=1, \ldots, T$. We obtain the following alternative formulation where we have a new unit procurement cost $\tilde{p_{t}}$ which accounts for the unit procurement cost $p_{t}$ and holding costs $\sum_{i=t}^{T} h_{i}, \tilde{p}_{t}=p_{t}+\sum_{i=t}^{T} h_{i}, \forall t=1, \ldots, T$. 


$$
\begin{array}{lll}
\min & \sum_{t=1}^{T}\left(r_{t} R+f_{t} y_{t}+\tilde{p}_{t} x_{t}+a_{t} A_{t}+b_{t} B_{t}-h_{t} \sum_{i=1}^{t} d_{i}\right) & \\
\text { s.t. } & \sum_{i=1}^{t} x_{i} \geq \sum_{i=1}^{t} d_{i} & \forall t=1, \ldots, T
\end{array}
$$

We obtain a new objective function in which $\sum_{t=1}^{T} h_{t} \sum_{i=1}^{t} d_{i}$ is a constant. Thus, we can eliminate the latter from the objective function (4.10). Constraints (4.2) and (4.7) of $s_{t}$ domain $\left(s_{t} \geq 0, \forall t=1, \ldots, T\right)$ are replaced with Constraints (4.11). Now, we will describe the idea of the dynamic programming formulation of this problem, noting that $D_{k, j}$ is the cumulative demand from period $k$ to period $j$ and $\mathbb{1}_{\beta}$ is an indicator function, defined as follows:

$$
\mathbb{1}_{\beta}= \begin{cases}1 & \text { if } \beta \text { is true } \\ 0 & \text { otherwise }\end{cases}
$$

\section{Stages}

t: period.

\section{State variables}

$i_{t}$ : Inventory level at the beginning of period $t$, where $i_{t} \in\left[0, D_{t, T}\right] \cap \mathbb{N}$. It is easy to see that the maximum quantity that can be stored at the beginning of $t$ can not exceed $\sum_{k=t}^{T} d_{k}$.

Decision variables

$x_{t}$ : Quantity to procure during period $t$, where $x_{t} \in\left[\max \left\{0, d_{t}-i_{t}\right\}, D_{t, T}-i_{t}\right] \cap \mathbb{N}$. It is clear that the lower bound of $x_{t}$ for a given state $i_{t}$ is $d_{t}-i_{t}$ if it is positive, otherwise, it is null. The upper bound of $x_{t}$ for a given state $i_{t}$ is $D_{t, T}-i_{t}$ to satisfy the demands from period $t$ to the last period $T$.

\section{Objective function}

$\phi_{t}\left(i_{t}, R\right)$ : optimal cost of satisfying the demand over periods $t, \ldots, T$ if the inventory level at the beginning of period $t$ is $i_{t}$ and $R$ is the reserved capacity, with $R \in\left[0, \max _{t=1, \ldots, T}\left\{\left\lceil\frac{D_{t, T}}{V_{t}}\right\rceil\right\}\right] \cap \mathbb{N}$. The maximum value of $R$ is equivalent to the greatest number of batches to replenish periodically.

\section{The backward recursive formulation would be:}

$$
\left\{\begin{array}{l}
\text { For } R=0, \ldots, \max _{t=1, \ldots, T}\left\{\left\lceil\frac{D_{t, T}}{V_{t}}\right\rceil\right\} \\
\text { For } i_{t}=0, \ldots, D_{t, T} \\
\phi_{t}\left(i_{t}, R\right)=\min _{x_{t}}\left\{r_{t} R+f_{t} \mathbb{1}_{x_{t}>0}+\tilde{p_{t}} x_{t}+a_{t}\left\lceil\frac{x_{t}}{V_{t}}\right\rceil \mathbb{1}_{\left\lceil\frac{x_{t}}{V_{t}}\right\rceil \leq R}+a_{t} R \mathbb{1}_{\left\lceil\frac{x_{t}}{V_{t}}\right\rceil>R}+\right. \\
\left.\qquad b_{t}\left(\left\lceil\frac{x_{t}}{V_{t}}\right\rceil-R\right) \mathbb{1}_{\left\lceil\frac{x_{t}}{V_{t}}\right\rceil>R}-h_{t} \sum_{i=1}^{t} d_{i}+\phi_{t+1}\left(i_{t}+x_{t}-d_{t}, R\right)\right\} \\
\text { Boundary conditions: } \phi_{T+1}\left(i_{T+1}, R\right)=0, \text { where } i_{T+1}=0 \\
\text { Objective: } \min _{R=0, \ldots, \max _{t=1, \ldots, T}\left\{\left\lceil\frac{D_{t, T}}{V_{t}}\right\rceil\right\}}\left\{\phi_{1}(0, R)\right\}
\end{array}\right.
$$

The total minimum cost of the LSP-B-CRC with variable $R$ over the entire planning horizon is $\min _{R=0, \ldots, \max _{t=1, \ldots, T}\left\{\left\lceil\frac{D_{t, T}}{V_{t}}\right\rceil\right\}}\left\{\phi_{1}(0, R)\right\}$ considering that, at the beginning of period 1 , the stock level is $i_{1}=0$. The complexity of this algorithm is in $O\left(T\left(D_{1, T}\right)^{3}\right)$ time. 


\subsubsection{LSP-B-CRC with time-dependent reserved capacity being decision vari- able}

In this problem, $R_{t}$ is a time-dependent decision variable (decision instant 1). In practical applications, this model is undesirable to use especially by the supplier, because, in some periods he can have big numbers of capacity to reserve, and in some others the retailer may not reserve any capacity, then the supplier, in this case, cannot manage the production of variable capacities. This model is desirable by the retailer because it specifies the reserved capacity he needs each period at a lower cost. We have two dominance properties.

Property 4.1. There exists an optimal solution such that, for any period $t$, the number of extra batches is null in the first instant of decision, $B_{t}=0, \forall t=1, \ldots, T$.

Having $r_{t}+a_{t} \leq b_{t}, \forall t=1, \ldots, T$, the retailer does not use batches ordered at a cost $b_{t}$ to determine the reserved capacity.

Property 4.2. There exists an optimal solution such that, for any period $t$, the reserved capacity is equal to the number of regular batches in the first instant of decision, $R_{t}=A_{t}, \forall t=1, \ldots, T$. The fact that we have in some periods positive reservation costs, $r_{t}>0$, requires not to reserve more than the number of batches replenished at a cost $a_{t}$.

We note that the values of $A_{t}$ and $B_{t}$ in the second instant of decision will be updated by respecting the values of $R_{t}$ decided in this stage and using the resolution method of LSP-B-CRC with fixed reserved capacity. These two properties are illustrated in Figure 4.3.

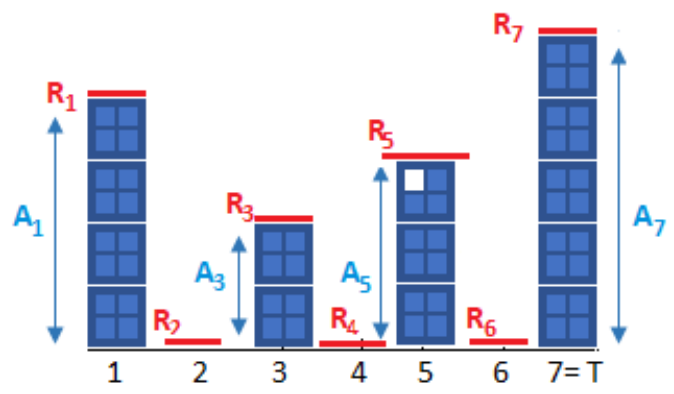

Figure 4.3: Possible values of $A_{t}$ and $R_{t}$ in LSP-B-CRC with time-dependent reserved capacity being a decision variable, $V=4$ and $T=7$

Thus, the model of LSP-B-CRC with time-dependent reserved capacity being a decision variable is simplified as follows:

$$
\begin{array}{lll}
\min & \sum_{t=1}^{T}\left(f_{t} y_{t}+p_{t} x_{t}+\left(a_{t}+r_{t}\right) R_{t}+h_{t} s_{t}\right) & \\
\text { s.t. } & \\
& x_{t} \leq R_{t} V_{t} & \forall t=1, \ldots, T
\end{array}
$$

This problem becomes the uncapacitated LSP with FTL cost structure. For time-dependent batch sizes, the problem is NP-hard and we can use the pseudo-polynomial algorithm of the previous problem without taking into account $R$ neither $b_{t}$. For a constant batch size, efficient algorithms from Li et al. (2004) and Akbalik and Rapine (2018) can be used to solve it (refer to Section 1.6.1). 


\subsection{LSP-B with several forms of CRC}

The specialized and general contracts of CRC have been proposed and developed in the literature, to assist supply chains in achieving better coordination and performance by aligning the objectives of supply chain members. In this section, these contracts will be integrated into the LSP with batch ordering. We identify the common parameters and decision variables of all types of CRC, and then we specify for each type a specific notation. Therefore, we consider the case where a retailer signs such a type of capacity reservation contract with a supplier to meet its deterministic demand $d_{t}$ of period $t$, over a planning horizon of $T$ periods. Therefore, the retailer orders, in batches of size $V_{t}$, a quantity $x_{t}$ at a unit purchase cost $p_{t}$. Recall that $y_{t}$ is the binary fixed ordering variable associated with the setup cost $f_{t}$, and $s_{t}$ is the stock level at the end of period $t$ with a holding cost $h_{t}$. We assume that the initial stock of the horizon is zero.

\subsubsection{LSP-B with deductible reservation contract}

The deductible reservation contract (DRC) is particularly attractive in high-tech industries such as semiconductors, electronics, and telecommunication equipments where the demand is volatile and the capacity is capital intensive (Jin and Wu, 2007; Erkoc and Wu, 2005).

In a DRC, the retailer reserves, for each period $t$, a future capacity $R_{t}$ (in terms of the total number of batches), with an upfront fee $r_{t}$ that will be deducted from the regular purchasing $\operatorname{cost} a_{t}$ for each batch replenished when the retailer places an order with $a_{t}>r_{t}$. Thus, before or at the beginning of the planning horizon (first instant of decision), the retailer pays in advance the amount of $\sum_{t=1}^{T} r_{t} R_{t}$. During the horizon (second instant of decision), in each period $t$, he orders $A_{t}$ batches being less than the capacity $R_{t}$ at a cost $a_{t}-r_{t}$ (unique feature of the DRC) and if the number of batches replenished exceeds this capacity, then he procures $B_{t}$ excess batches at a cost $a_{t}$. When the retailer purchases all the reserved batches, the reservation fee is deducted from the regular order cost. However, if the reserved batches are not fully purchased, the reservation fee associated with unused capacity is not refundable. The batch quantities of reservation and ordering in the DRC are illustrated in Figure 4.4.

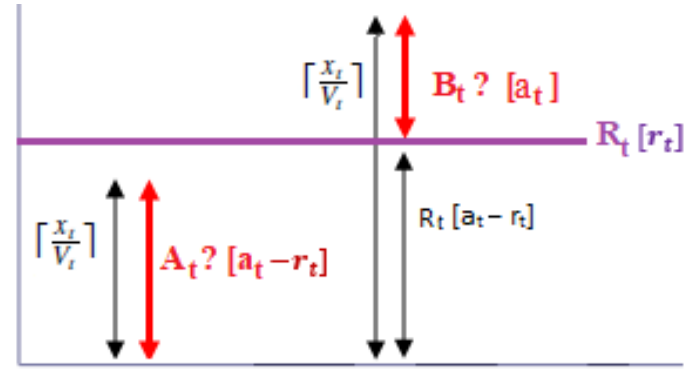

Figure 4.4: Illustration of the principle of DRC at a given period $t$ at the 2 nd decision level

The ordering cost $q_{t}\left(x_{t}\right)$ that the retailer pays in period $t$ for $x_{t}$ units replenished is considered as follows (see Figure 4.4 to compute the values of $A_{t}$ and $B_{t}$ ):

$$
q_{t}\left(x_{t}\right)=\left\{\begin{array}{lll}
f_{t}+p_{t} x_{t}+\left(a_{t}-r_{t}\right)\left\lceil\frac{x_{t}}{V_{t}}\right\rceil+r_{t} R_{t} & \text { if } 0<\left\lceil\frac{x_{t}}{V_{t}}\right\rceil \leq R_{t} & A_{t}=\left\lceil\frac{x_{t}}{V_{t}}\right\rceil \\
f_{t}+p_{t} x_{t}+a_{t}\left\lceil\frac{x_{t}}{V_{t}}\right\rceil & \text { if } R_{t} \leq\left\lceil\frac{x_{t}}{V_{t}}\right\rceil & B_{t}=\left\lceil\frac{x_{t}}{V_{t}}\right\rceil-R_{t}
\end{array}\right.
$$

First, the retailer solves the LSP-B under deductible reservation contract (LSP-B-DRC) at the tactical level in which he determines the optimal numbers of $R_{t}$ over the horizon of $T$ periods 
using the first demand forecasts. Second, he solves the LSP-B-DRC at the operational level in which he determines the optimal numbers of $A_{t}$ and $B_{t}$ by using the revised (or second) forecasts of demand.

We model the LSP-B-DRC at the tactical level because it considers the capacity reservation decision in addition to the procurement and inventory decisions. At operational level, the reserved capacity becomes a known parameter. The general formulation of LSP-B-DRC as an MILP is the same one proposed for LSP-B-CRC (4.1)-(4.9) by replacing $a_{t}$ with $a_{t}-r_{t}$ and $b_{t}$ with $a_{t}$, $\forall t=1, \ldots, T$. Therefore, the objective function of LSP-B-DRC is $\min \sum_{t=1}^{T}\left(r_{t} R_{t}+f_{t} y_{t}+p_{t} x_{t}+\right.$ $\left.\left(a_{t}-r_{t}\right) A_{t}+a_{t} B_{t}+h_{t} s_{t}\right)$.

The tactical LSP-B-DRC under time-varying or stationary reserved capacity being decision variable aims to minimize $\sum_{t=1}^{T}\left(f_{t} y_{t}+p_{t} x_{t}+a_{t} R_{t}+h_{t} s_{t}\right)$. We note that instead of explicitly using the reservation fee $r_{t}$ in the objective function, we use the regular procurement cost $a_{t}$. The reason is because we have $a_{t}>r_{t}$, which means that we have $a_{t}=r_{t}+\lambda_{t}$ with $\lambda_{t}>0$. Hence, procurement decisions are intimately related to capacity reservation decisions, because, if we only use the reservation fee $r_{t}$, we can reserve, in period $t$, a large capacity $R_{t}$ which is rendered idle because the procurement cost $a_{t}$ is very expensive. Thus, the cost of capacity reservation must be balanced with the costs of procurements and inventories. This problem is equivalent to the tactical LSP-B-CRC presented in Sections 4.2.2 and 4.2.3. The complexity and the resolution method for the operational LSP-B-DRC are mentioned in Section 4.2.1.

\subsubsection{LSP-B with take-or-pay contract}

The Take-or-pay contract (TOPC) is prevalent in natural gas industries (Brooke, 1992; Marple and Roland, 1989), electric utility coal market (Joskow, 1987), companies producing aluminum from petroleum coke (Goldberg and Erickson, 1987) and municipalities and waste incineration facilities (Brooke, 1992).

In a TOPC, the retailer reserves, for each period $t$, a capacity $R_{t}$ relying on the first demand forecasts, but, makes no payment to the supplier upfront. In the next step, when its demand is revised, the supplier requires the retailer to either purchase a number of batches more than the capacity threshold, $R_{t}^{\prime}=\left\lceil\left(1-\psi_{t}\right) R_{t}\right\rceil$ batches at a cost $a_{t}$ ('take'), or pay a penalty cost $p_{t}^{n}$ for each unused threshold batch if the number of batches replenished falls below $R_{t}^{\prime}$ ('pay'). This contract specifies in each period $t$ both the penalty cost being lower than the regular procurement $\operatorname{cost} a_{t}\left(p_{t}^{n}<a_{t}\right)$ and the flexibility rate $\psi_{t}$ with $0 \leq \psi_{t} \leq 1$, in other words, $1-\psi_{t}$ is the threshold rate. Let $A_{t}$ be the number of batches ordered in period $t$ below the threshold $R_{t}^{\prime}$, and $B_{t}$ be the number of additional batches ordered above the same threshold (see Figure 4.5). Therefore, there is no obligation to purchase a minimum number of batches.

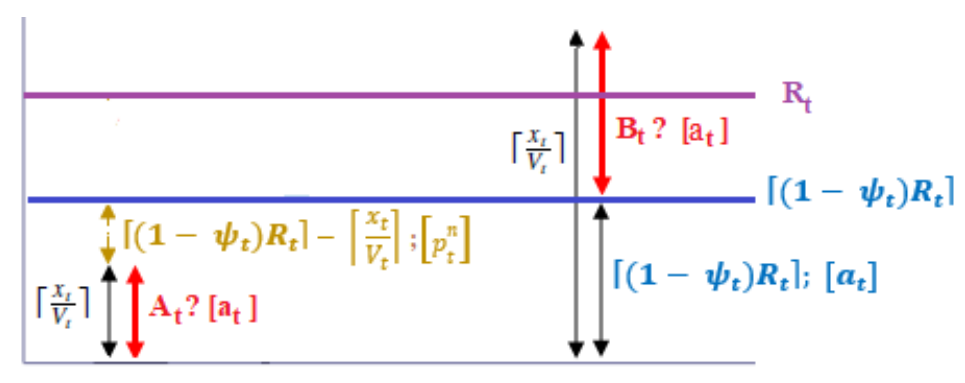

Figure 4.5: Illustration of the principle of TOPC at a given period $t$ at the 2 nd decision level

The retailer orders, in period $t$, a positive quantity of $x_{t}$ units in $A_{t}+B_{t}$ batches and pays 
the following cost $q_{t}\left(x_{t}\right)$ (refer to Figure 4.5):

$$
q_{t}\left(x_{t}\right)=\left\{\begin{array}{lll}
f_{t}+p_{t} x_{t}+a_{t}\left\lceil\frac{x_{t}}{V_{t}}\right\rceil+p_{t}^{n}\left(R_{t}^{\prime}-\left\lceil\frac{x_{t}}{V_{t}}\right\rceil\right) & \text { if } 0<\left\lceil\frac{x_{t}}{V_{t}}\right\rceil \leq R_{t}^{\prime} & A_{t}=\left\lceil\frac{x_{t}}{V_{t}}\right. \\
f_{t}+p_{t} x_{t}+a_{t}\left\lceil\frac{x_{t}}{V_{t}}\right\rceil & \text { if } R_{t}^{\prime} \leq\left\lceil\frac{x_{t}}{V_{t}}\right\rceil & B_{t}=\left\lceil\frac{x_{t}}{V_{t}}\right\rceil-R_{t}^{\prime}
\end{array}\right.
$$

The TOPC with $\psi_{t}=0$ can be implemented as a DRC. We formulate the LSP-B-TOPC as an MILP knowing that it can be used at either tactical level or operational level with fixed $R_{t}^{\prime}$ :

$$
\begin{array}{lll}
\min & \sum_{t=1}^{T}\left(p_{t}^{n} R_{t}^{\prime}+f_{t} y_{t}+p_{t} x_{t}+\left(a_{t}-p_{t}^{n}\right) A_{t}+a_{t} B_{t}+h_{t} s_{t}\right) & \\
\text { s.t. } & & \\
& \left(1-\psi_{t}\right) R_{t} \leq R_{t}^{\prime} & \forall t=1, \ldots, T \\
& A_{t} \leq R_{t}^{\prime} & \forall t=1, \ldots, T \\
& \forall t=1, \ldots, T
\end{array}
$$

The objective function (4.14) is obtained by minimizing the procurement, penalty and inventory costs as follows: $\sum_{t=1}^{T}\left(f_{t} y_{t}+p_{t} x_{t}+a_{t} A_{t}+p_{t}^{n}\left(R_{t}^{\prime}-A_{t}\right)+a_{t} B_{t}+h_{t} s_{t}\right)$. Constraints (4.15) determine the values of reserved capacity $R_{t}$ after finding the optimal values of the capacity threshold $R_{t}^{\prime}$. Constraints (4.16) limit the number of batches $A_{t}$ by the threshold. Constraints (4.17) are the feasibility domain of the decision variable $R_{t}^{\prime}$.

We note that the LSP-B-TOPC is a LSP-B-CRC with a reservation fee $p_{t}^{n}$, a reservation capacity $R_{t}^{\prime}$, a regular procurement cost $a_{t}-p_{t}^{n}$ and a particular procurement cost of an excess batch $a_{t}$.

\subsubsection{LSP-B with pay-to-delay contract}

A pay-to-delay contract (PTDC) is used in the semiconductor industries (Brown and Lee, 1997). It has been offered by the Taiwanese Semiconductor Manufacturing Company, a semiconductor fabrication foundry company.

Under a PTDC, the retailer makes two procurement decisions. Before the selling season begins, the retailer should decide, in each period $t$, to purchase the minimum commitment $K_{t}$ at an upfront cost $\kappa_{t}$ per batch, and reserve additional batches representing the option capacity $R_{t}=Z_{t}-K_{t}$ with an upfront cost $r_{t}$ per batch knowing that $Z_{t}$ is the capacity of commitment and options. Later, $A_{t}$ additional batches (number of options exercised) up to the option capacity $R_{t}$ may be purchased at an extra cost $a_{t}$ with $a_{t}+r_{t}>\kappa_{t}$. The retailer can also purchase $B_{t}$ additional unreserved batches at a cost $b_{t}$ with $b_{t}>a_{t}+r_{t}$ (see Figure 4.6). Then, he makes his final decision to compute, in each period $t$, the number of batches ordered at a cost $a_{t}$ and $b_{t}$.

The retailer pays the cost $q_{t}\left(x_{t}\right)$ in a PTDC with $x_{t}$ being the positive purchased units (see Figure 4.6):

$$
q_{t}\left(x_{t}\right)=\left\{\begin{array}{lll}
r_{t} R_{t}+f_{t}+p_{t} x_{t}+\kappa_{t} K_{t}+a_{t}\left(\left\lceil\frac{x_{t}}{V_{t}}\right\rceil-K_{t}\right) & \text { if } K_{t} \leq\left\lceil\frac{x_{t}}{V_{t}}\right\rceil \leq Z_{t} & A_{t}=\left\lceil\frac{x_{t}}{V_{t}}\right\rceil-K_{t} \\
r_{t} R_{t}+f_{t}+p_{t} x_{t}+\kappa_{t} K_{t}+a_{t} R_{t}+b_{t}\left(\left\lceil\frac{x_{t}}{V_{t}}\right\rceil-Z_{t}\right) & \text { if } Z_{t} \leq\left\lceil\frac{x_{t}}{V_{t}}\right\rceil & B_{t}=\left\lceil\frac{x_{t}}{V_{t}}\right\rceil-Z_{t}
\end{array}\right.
$$

The formulation of the LSP-B-PTDC at tactical level is stated below knowing that it can be used for the operational problem with fixed $R_{t}$ and $K_{t}$ values:

$$
\begin{array}{lll}
\min _{\text {s.t. }} & \sum_{t=1}^{T}\left(r_{t} R_{t}+f_{t} y_{t}+p_{t} x_{t}+\kappa_{t} K_{t}+a_{t} A_{t}+b_{t} B_{t}+h_{t} s_{t}\right) & \\
& K_{t} V_{t} \leq x_{t} \leq\left(K_{t}+A_{t}+B_{t}\right) V_{t} & \forall t=1, \ldots, T \\
& K_{t} \in \mathbb{N} & \forall t=1, \ldots, T
\end{array}
$$




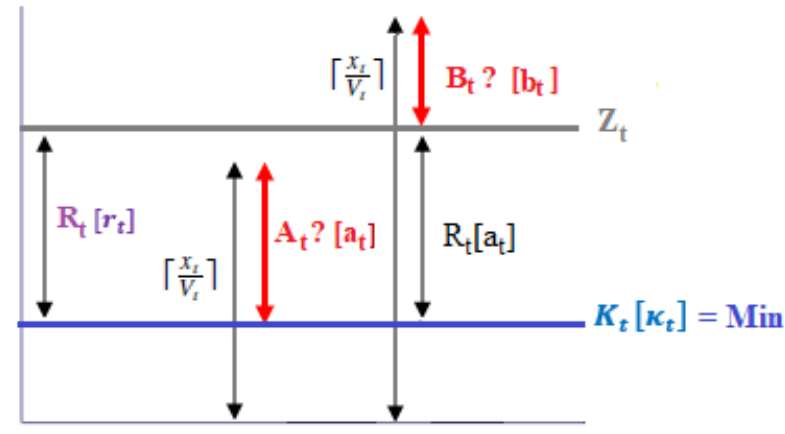

Figure 4.6: Illustration of the principle of PTDC at a given period $t$ at the 2 nd decision level

The objective function (4.18) aims to minimize the procurement of commitments with different costs, reservation of options and inventory costs. Constraints (4.19) precise the lower bound of quantities purchased in batches. Constraints (4.20) are the feasibility domain of the decision variable $K_{t}$. The LSP-B-PTDC is a LSP-B-CRC with minimum commitments. LSP-B-PTDC with $K_{t}=0, \forall t=1, \ldots, T$ is equivalent to LSP-B-CRC.

\subsubsection{LSP-B with quantity flexibility contract}

The quantity flexibility contract (QFC) has appeared in various industries where demand uncertainties and price fluctuations are frequent. It was adopted by IBM, Sun Microsystems, Solectron, Hewlett Packard, Toyota, Nippon Otis (Tsay, 1999; Tsay and Lovejoy, 1999), and Wistron Corporation, one of world's largest personal computer contract manufacturers (Chung et al., 2010).

In the QFC, the retailer decides to order at two distinct time instants, one when he uses demand forecasts, and another when he revises its demand forecasts. At the first instant, the retailer orders, for each period $t$ from the supplier, $R_{t}$ initial commitment batches at a price $a_{t}$. Thus, the first decision concerns the variables $R_{t}, \forall t=1, \ldots, T$ in addition to the variables cited in Section 4.3. The first problem is at the tactical level.

In the second instant, after observing the demand for a period $t$, the retailer commits to purchase a number of batches no less than a certain percentage $\beta_{t}$ below the initial commitment batches $R_{t}$, namely $\left\lceil\left(1-\beta_{t}\right) R_{t}\right\rceil$ batches, and the supplier guarantees to deliver a number of batches up to a certain percentage $\alpha_{t}$ above the initial commitment $R_{t}$, thus $\left\lceil\left(1+\alpha_{t}\right) R_{t}\right\rceil$ batches. When the number of batches ordered in period $t$ is less than $R_{t}$, the retailer pays a cost of $a_{t}$ per batch. Otherwise, a cost $b_{t}$ is paid for each excess batch with $b_{t}>a_{t}$. Therefore, in the second decision, the retailer determines the number of batches to replenish from the minimum commitment batch level, $\left\lceil\left(1-\beta_{t}\right) R_{t}\right\rceil$, until $R_{t}$, denoted by $A_{t}$, and the number of batches $B_{t}$ between $R_{t}$ and the maximum commitment batch level $\left\lceil\left(1+\alpha_{t}\right) R_{t}\right\rceil$. The second problem arises at the operational level (see Figure 4.7).

The ordering cost $q_{t}\left(x_{t}\right)$ of LSP-B-QFC, in period $t$, for $x_{t}$ units replenished in $\left\lceil\left(1-\beta_{t}\right) R_{t}\right\rceil+$ $A_{t}+B_{t}$ batches is considered as follows (see Figure 4.8):

$q_{t}\left(x_{t}\right)=\left\{\begin{array}{lll}f_{t}+p_{t} x_{t}+a_{t}\left\lceil\frac{x_{t}}{V_{t}}\right\rceil & \text { if }\left\lceil\left(1-\beta_{t}\right) R_{t}\right\rceil \leq\left\lceil\frac{x_{t}}{V_{t}}\right\rceil \leq R_{t} & A_{t}=\left\lceil\frac{x_{t}}{V_{t}}\right\rceil-\left\lceil\left(1-\beta_{t}\right) R_{t}\right\rceil \\ f_{t}+p_{t} x_{t}+a_{t} R_{t}+b_{t}\left(\left\lceil\frac{x_{t}}{V_{t}}\right\rceil-R_{t}\right) & \text { if } R_{t} \leq\left\lceil\frac{x_{t}}{V_{t}}\right\rceil \leq\left\lceil\left(1+\alpha_{t}\right) R_{t}\right\rceil & B_{t}=\left\lceil\frac{x_{t}}{V_{t}}\right\rceil-R_{t}\end{array}\right.$

The QFC cost structure in the second decision (see Figure 4.8) is similar to the one of the 


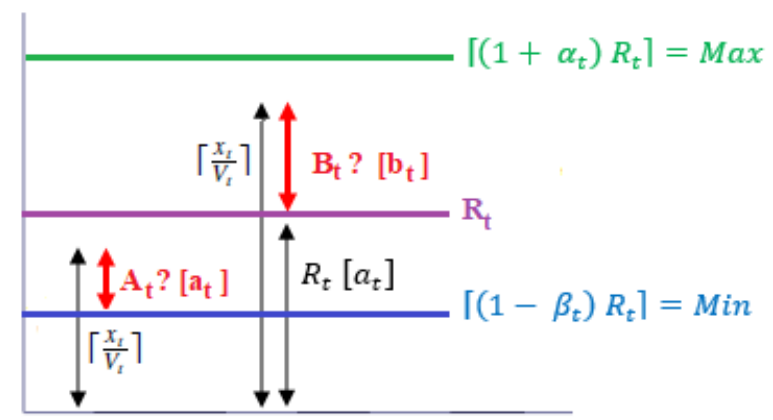

Figure 4.7: Illustration of the principle of QFC at a given period $t$ at the 2 nd decision level

capacity reservation contract with fixed reserved capacity (see Figure 1.8 depicted in Chapter 1 ) but with minimum and maximum commitments of batches replenished.

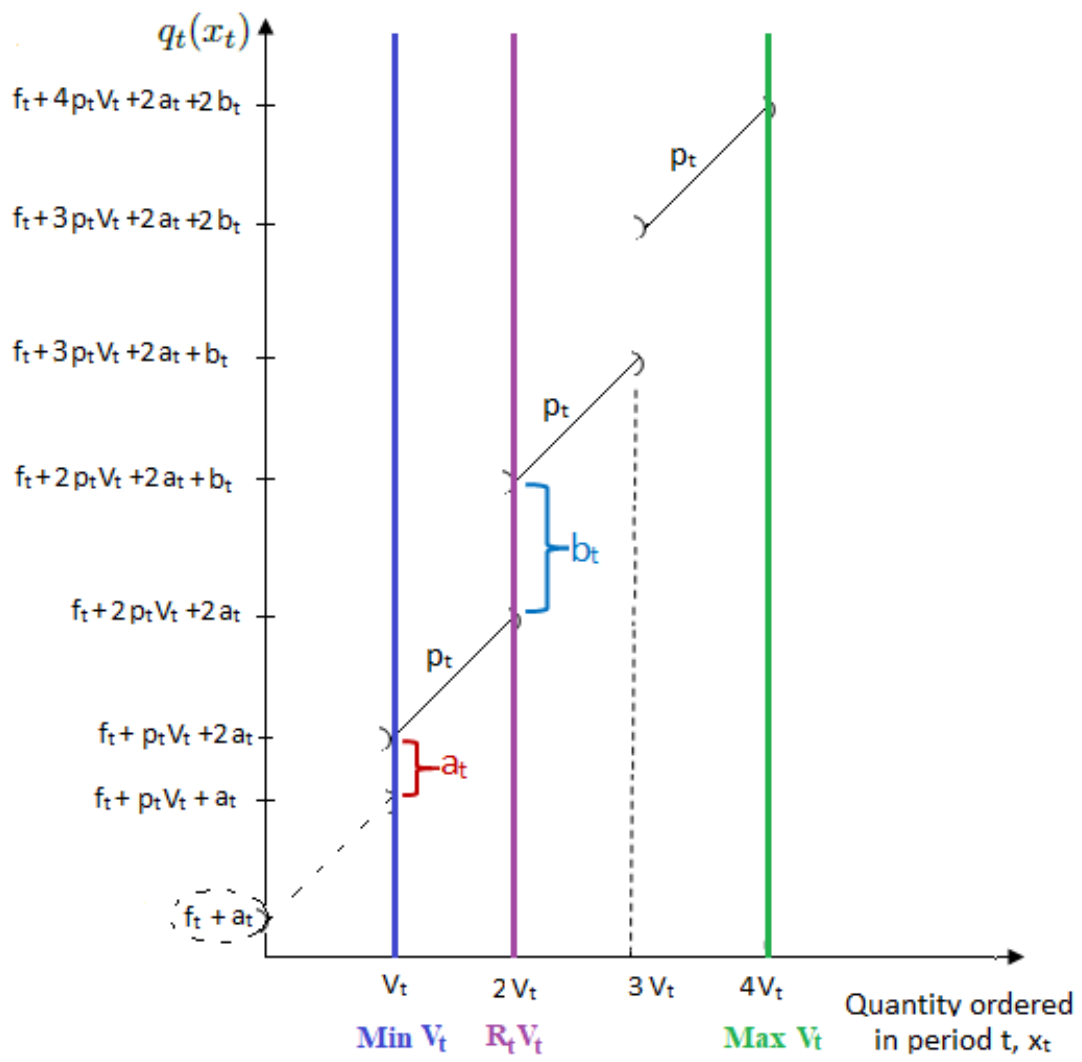

Figure 4.8: QFC cost structure at the 2nd decision level

A MILP formulation of the LSP-B-QFC is stated as follows:

$$
\begin{array}{lll}
\min & \sum_{t=1}^{T}\left(f_{t} y_{t}+p_{t} x_{t}+a_{t} R_{t}^{\prime}+a_{t} A_{t}+b_{t} B_{t}+h_{t} s_{t}\right) & \\
\text { s.t. } & & (4.2)-(4.3) \\
& \left(1-\beta_{t}\right) R_{t} \leq R_{t}^{\prime} & \forall t=1, \ldots, T \\
& R_{t}^{\prime} V_{t} \leq x_{t} \leq\left(R_{t}^{\prime}+A_{t}+B_{t}\right) V_{t} & \forall t=1, \ldots, T \\
& A_{t} \leq R_{t}-R_{t}^{\prime} & \forall t=1, \ldots, T
\end{array}
$$




$$
\begin{array}{ll}
\left(1+\alpha_{t}\right) R_{t} \leq R_{t}^{\prime \prime} & \forall t=1, \ldots, T \\
B_{t} \leq R_{t}^{\prime \prime}-R_{t} & \forall t=1, \ldots, T \\
R_{t}^{\prime}, R_{t}^{\prime \prime} \in \mathbb{N} & \forall t=1, \ldots, T
\end{array}
$$

Constraints (4.22) determine the number of batches of minimum commitment. Constraints (4.23) respect the minimum commitment and specify the batch replenishment of both costs. Constraints (4.24) are for limiting the number of batches $A_{t}$. Constraints (4.25) determine the number of batches of maximum commitment. Constraints (4.26) are for limiting the number of batches $B_{t}$.

We note that the LSP-B-QFC with $\beta_{t}=1$ and $\alpha_{t}=+\infty, \forall t=1, \ldots, T$, coincides with the LSP-B-CRC with null reservation costs.

\subsubsection{LSP-B with backup agreement}

The backup agreements (BA) are common in the apparel catalog industries. Eppen and Iyer (1997) reported that the catalog company Catco had adopted such contracts with its manufacturers named Anne Klein, Finity, DKNY, and Liz Claiborne to deal with demand uncertainty by providing flexibility to Catco during the season of replenishment.

In a model of a backup agreement, the retailer makes two decisions in two different times. In the first decision (for example before or at the beginning of the ordering season), he makes a commitment to purchase $R_{t}$ batches in every period $t$ at a cost $a_{t}, \forall t \in\{1, \ldots, T\}$. The supplier initially delivers for period $t$ a number of batches containing a certain percentage $\omega_{t}$ below $R_{t}$ $\left(\left\lceil\left(1-\omega_{t}\right) R_{t}\right\rceil\right.$ batches$)$, and agrees to reserve $R_{t}-\left\lceil\left(1-\omega_{t}\right) R_{t}\right\rceil$ batches. The LSP-B with BA, denoted by LSP-B-BA, in the first instant of decision is the LSP-B-CRC at the same level of decision.

In the second instant of decision (during the ordering season), the retailer may purchase additional batches up to the number of reserved batches $R_{t}-\left\lceil\left(1-\omega_{t}\right) R_{t}\right\rceil$ at the same price $a_{t}$. Let $A_{t}$ be the number of excess batches ordered from the minimum commitment $\left\lceil\left(1-\omega_{t}\right) R_{t}\right\rceil$ until $R_{t}$, in period $t$. If the retailer decides to purchase $A_{t}$ batches in period $t$, where $A_{t}<$ $R_{t}-\left\lceil\left(1-\omega_{t}\right) R_{t}\right\rceil$, then he pays a penalty, $p_{t}^{n}$ for any reserved batch not purchased. The principle of $\mathrm{BA}$ in the second decision instant at a given period $t$ is shown in Figure 4.9 .

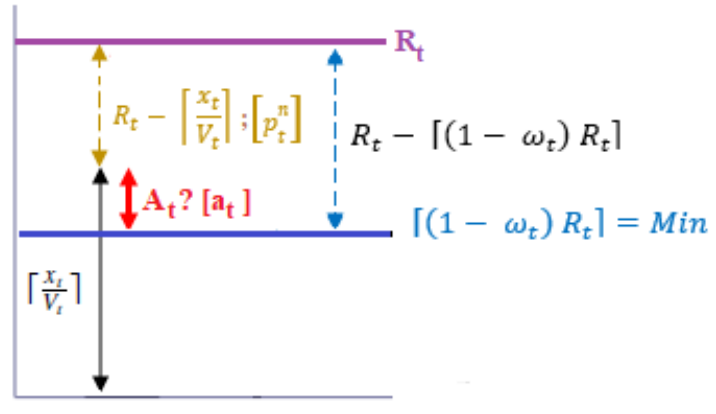

Figure 4.9: Illustration of the principle of BA at a given period $t$ at the 2 nd decision level

Replenishing a positive amount $x_{t}$, in LSP-B-BA, incurs the following ordering cost $q_{t}\left(x_{t}\right)$ in period $t$ :

$$
q_{t}\left(x_{t}\right)=f_{t}+p_{t} x_{t}+a_{t}\left\lceil\frac{x_{t}}{V_{t}}\right\rceil+p_{t}^{n}\left(R_{t}-\left\lceil\frac{x_{t}}{V_{t}}\right\rceil\right) \quad \text { if }\left\lceil\left(1-\omega_{t}\right) R_{t}\right\rceil \leq\left\lceil\frac{x_{t}}{V_{t}}\right\rceil \leq R_{t}, \quad A_{t}=\left\lceil\frac{x_{t}}{V_{t}}\right\rceil-\left\lceil\left(1-\omega_{t}\right) R_{t}\right\rceil
$$

In period $t$, if we have $a_{t} \geq p_{t}^{n}$, then the $\mathrm{BA}$ procurement cost structure is presented in Figure 4.10 and, it is equivalent to the one of the CRC with fixed reserved capacity, but with 
minimum commitments of batches replenished and $b_{t}=+\infty$. If we have $a_{t}<p_{t}^{n}$, then the BA procurement cost structure is depicted in Figure 4.11

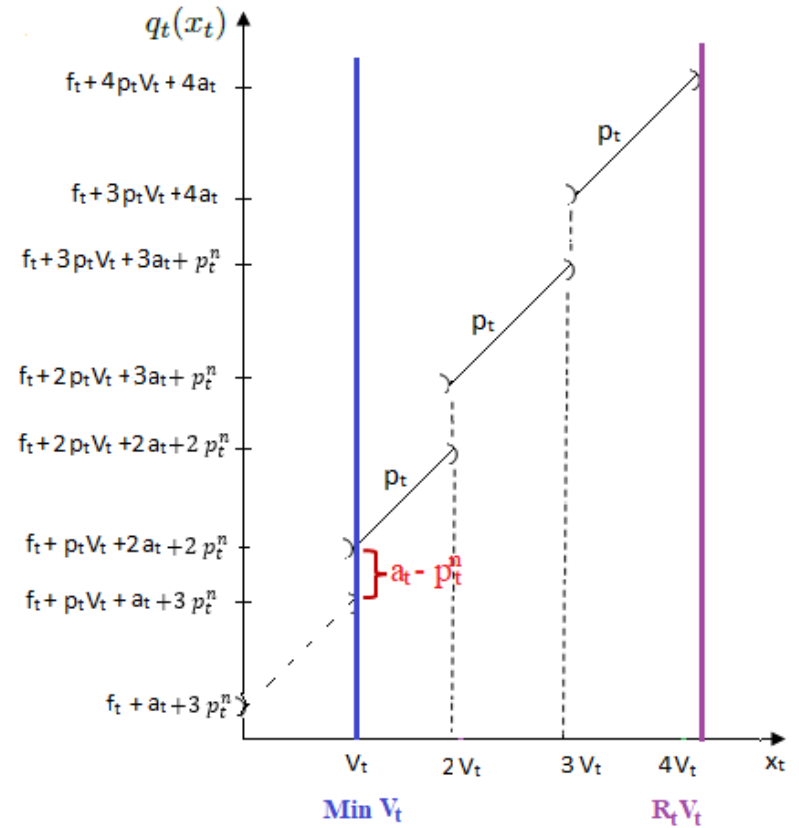

Figure 4.10: BA cost structure if $a_{t} \geq p_{t}^{n}$

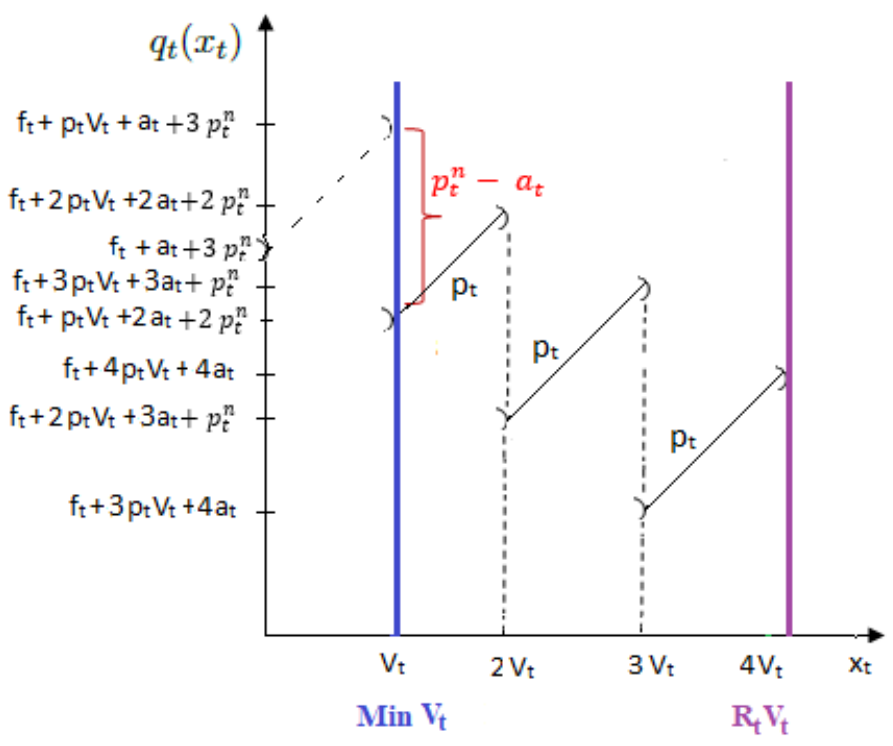

Figure 4.11: BA cost structure if $a_{t}<p_{t}^{n}$

The MILP formulation of the LSP-B-BA is stated below. Its objective function aims at minimizing the procurement and inventory costs of units and batches, $\sum_{t=1}^{T}\left(f_{t} y_{t}+p_{t} x_{t}+a_{t} R_{t}^{\prime}+\right.$ $\left.a_{t} A_{t}+p_{t}^{n}\left(R_{t}-R_{t}^{\prime}-A_{t}\right)+h_{t} s_{t}\right)$ with $R_{t}^{\prime}=\left\lceil\left(1-\omega_{t}\right) R_{t}\right\rceil$ to obtain the new one (4.28).

$$
\begin{array}{lll}
\min _{\text {s.t. }} & \sum_{t=1}^{T}\left(p_{t}^{n} R_{t}+f_{t} y_{t}+p_{t} x_{t}+\left(a_{t}-p_{t}^{n}\right) R_{t}^{\prime}+\left(a_{t}-p_{t}^{n}\right) A_{t}+h_{t} s_{t}\right) & \\
& R_{t}^{\prime} V_{t} \leq x_{t} \leq\left(R_{t}^{\prime}+A_{t}\right) V_{t} & \forall t=1, \ldots, T
\end{array}
$$

Constraints (4.29) are similar to Constraints (4.23) with $B_{t}=0$. The LSP-B-BA with $a_{t} \geq p_{t}^{n}, \forall t=1, \ldots, T$, is the LSP-B-CRC with minimum commitment and $b_{t}=+\infty$.

\subsubsection{LSP-B with minimum commitment contract}

The minimum commitment contracts (MCC) are a common practice in the electronics industry in which the demands are considered to be random. There exist several works analyzing the MCC, see for example, Anupindi and Akella (1993), Katz et al. (1994), and Bassok and Anupindi (1997).

In the MCC, at the beginning of the horizon (first decision instant), the retailer specifies to the supplier the minimum number of batches $K_{t}$ to be purchased for each period $t$, for $t=1, \ldots, T$. 
The supplier provides, in each period $t$, a menu of $n$ pairs of discount percentage $\epsilon_{i}$ applied on the regular batch cost $a_{t}$, and minimum commitment batches $k_{i},\left(\epsilon_{i}, k_{i}\right) \forall i=1, \ldots, N$, from which the retailer chooses the optimal one. In each period $t$, we associate for each pair $i$ a binary decision variable $z_{t, i}$ as follows:

$$
z_{t, i}= \begin{cases}1 & \text { if the } i^{t h} \text { pair }\left(\epsilon_{i}, k_{i}\right) \text { is chosen in period } t \\ 0 & \text { otherwise }\end{cases}
$$

Thus, the retailer must determine the number of batches $A_{t, i}$ to purchase in each period $t$ with the $i^{t h}$ pair $\left(\epsilon_{i}, k_{i}\right)$ in order to satisfy the first demand forecast and to specify the values of $K_{t}$ which will be used as given parameters in the second decision instant. The batch procurement cost in period $t$ has an all-units discount structure, as shown on Figure 4.12 in which as the minimum commitment $k_{i}$ increases, the reduction percentage $\epsilon_{i}$ would rise.

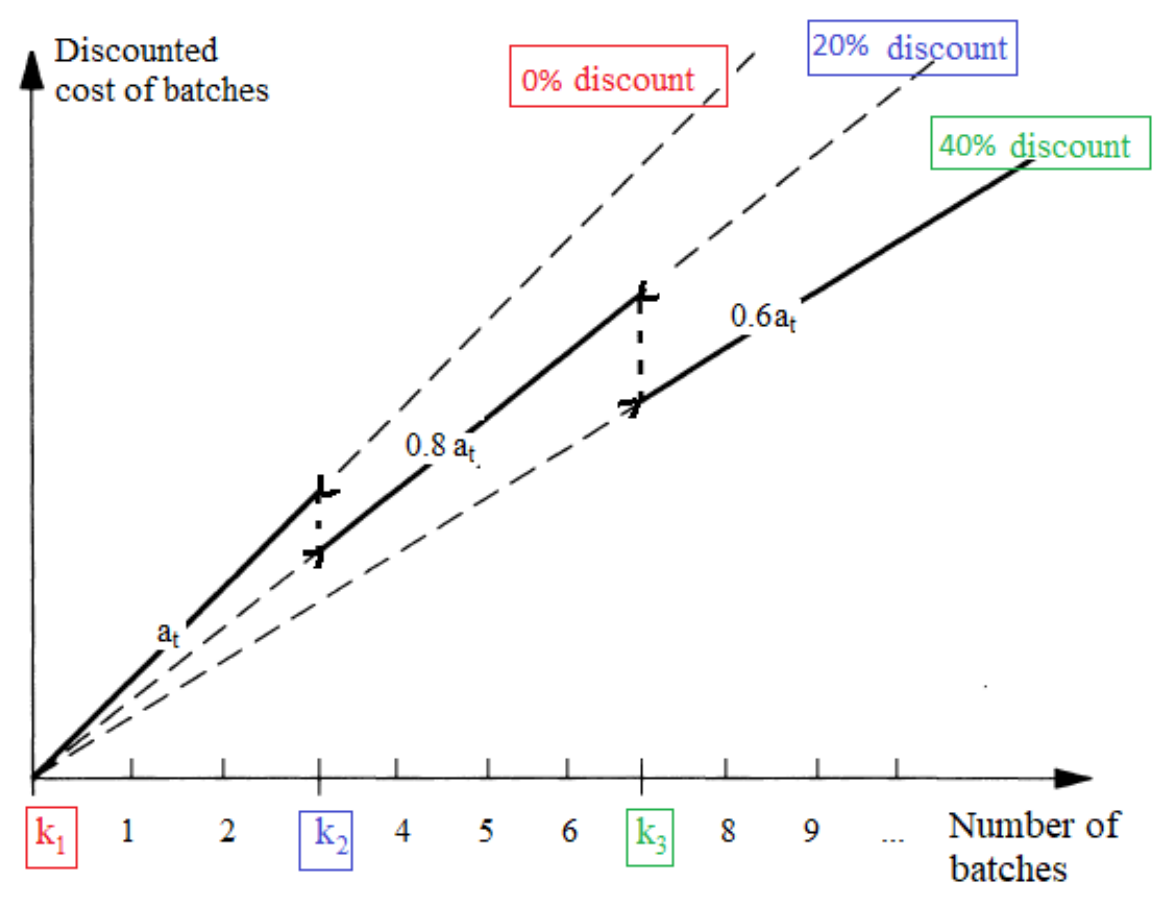

Figure 4.12: Cost of batches at a given period $t$

In the first instant of decision of LSP-B-MCC, if the retailer decides to order a positive amount $x_{t}$, then he must pay the following ordering cost $q_{t}\left(x_{t}\right)$ in period $t$ :

$$
\begin{aligned}
& q_{t}\left(x_{t}\right)=f_{t}+p_{t} x_{t}+\pi_{t}\left(x_{t}\right) \text { with }
\end{aligned}
$$

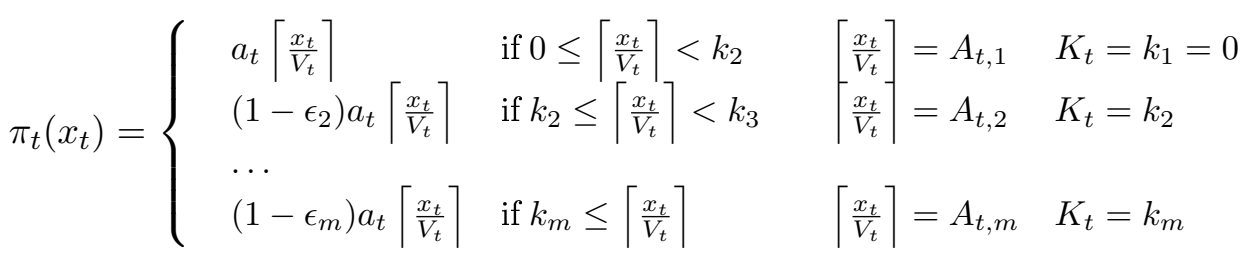

The MCC cost structure in the first decision is presented in Figure 4.13 in which $((1-$ $\left.\left.\epsilon_{2}\right)-k_{2} \epsilon_{2}\right) a_{t}=\pi_{t}\left(\left(k_{2}+1\right) V_{t}\right)-k_{2} a_{t}$, which means that this last value is equal to the difference between the procurement cost of $k_{2}+1$ batches with the cost $\pi_{t}$ and the procurement cost of $k_{2}$ 
batches with the cost $a_{t}$. This value can be either non-negative or non-positive. We consider, in Figure 4.13 , that this latter is positive. In the same way, $\left(\left(1-\epsilon_{3}\right)-k_{3}\left(\epsilon_{3}-\epsilon_{2}\right)\right) a_{t}$ is equal to $\pi_{t}\left(\left(k_{3}+1\right) V_{t}\right)-k_{3}\left(1-\epsilon_{2}\right) a_{t}$, and is considered to be negative.

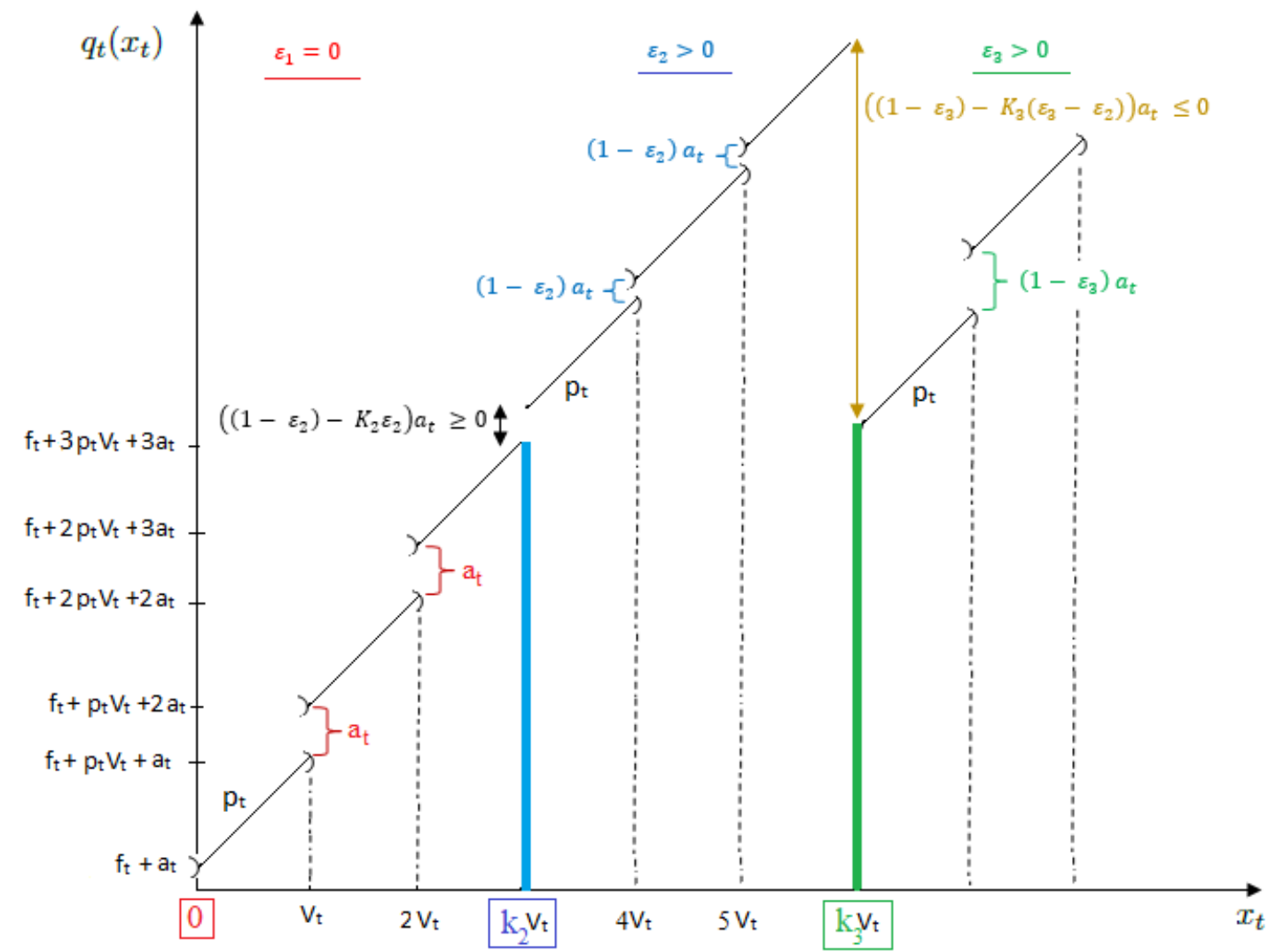

Figure 4.13: MCC cost structure at the 1st decision level

The LSP-B-MCC in the first instant of decision is formulated as the following MILP:

$$
\begin{array}{ll}
\min & \sum_{t=1}^{T}\left(f_{t} y_{t}+p_{t} x_{t}+\left(\sum_{i=1}^{n}\left(1-\epsilon_{i}\right) a_{t} A_{t, i}\right)+h_{t} s_{t}\right) \\
\text { s.t. } & k_{i} z_{t, i} \leq A_{t, i} \leq\left(k_{i+1}-1\right) z_{t, i} \\
& \sum_{i=1}^{n} z_{t, i}=1 \\
& x_{t} \leq\left(\sum_{i=1}^{n} A_{t, i}\right) V_{t} \\
& K_{t}=\sum_{i=1}^{n} k_{i} z_{t, i} \\
& s_{0}=0 \\
& A_{t, i}, K_{t} \in \mathbb{N} \\
& z_{t, i} \in\{0,1\}
\end{array}
$$

Constraints (4.31) mean that if $z_{t, i}=0$, then $A_{t, i}=0$, and if $z_{t, i}=1$, then the number of batches replenished in period $t$ with the $i^{t h}$ pair $\left(\epsilon_{i}, k_{i}\right)$ is positive and it does not reach the minimum commitment of the next pair $k_{i+1}$. Constraints (4.32) affirm that there is exactly one pair chosen in period $t$. Constraints (4.33) ensure the replenishment of $x_{t}$ in batches. Constraints (4.34) identify the value of the optimal minimum commitment in each period $t$.

In the second instant of decision, the retailer has to place in each period $t$ an order of $A_{t}$ batches at a cost $a_{t}^{\prime}$ such that the number of batches $A_{t}$ is greater than or equal to the minimum 
purchase agreement $K_{t}$, and $a_{t}^{\prime}=\sum_{i=1}^{n}\left(1-\epsilon_{i}\right) z_{t, i} a_{t}$. The values of $K_{t}$ and $z_{t, i}, \forall t=1, \ldots, T$ and $\forall i=1, \ldots, n$ are obtained from the first decision of LSP-B-MCC. The LSP-B-MCC at the operational level becomes an LSP-B with lower limits on batches to replenish.

We note that the whole LSP-B-MCC with $i=1$ is equivalent to the LSP-B-CRC with null reservation fees, $r_{t}=0, \forall t=1, \ldots, T$ and $b_{t}=a_{t}, \forall t=1, \ldots, T$.

\subsubsection{LSP-B with revenue-sharing contract}

The revenue-sharing contract (RSC) is prevalent in the videocassette rental industry as shown by Cachon and Lariviere (2005).

In a RSC, the retailer orders, in each period $t$, units in $A_{t}$ full batches at a low cost $a_{t}$. In return, the supplier shares a fraction $(1-\phi)$ of the retailer's total revenue with $0 \leq \phi \leq 1$. The retailer sells the demand $d_{t}$ with a unit selling price $v_{t}\left(v_{t} \geq p_{t}+\frac{a_{t}}{V_{t}}\right)$ and earns a unit revenue $p_{t}^{b}$ for the salvaged units $q_{t}$ by assuming that the leftover inventory in each period $t$ is salvaged. Hence, the total revenue of the retailer before sharing it, in each period $t$, is equal to $v_{t} d_{t}+p_{t}^{b} q_{t}$. Therefore, in addition to the total procurement cost of units and batches $\left(f_{t}, x_{t}\right.$, and $\left.a_{t}\right)$, the supplier receives the cost $(1-\phi) v_{t} d_{t}+(1-\phi) p_{t}^{b} q_{t}$. The retailer keeps $\phi v_{t} d_{t}+\phi p_{t}^{b} q_{t}$ as his new revenue.

The retailer maximizes his total profit in order to find the optimal plan over the horizon. The profit is obtained by subtracting the total costs of procurement, inventory holding and revenue sharing from the total realized revenue. The resulting mixed integer program of LSP-B-RSC is given below, in which we provide a minimization of - retailer profit:

$$
\begin{array}{lll}
\min & \sum_{t=1}^{T}\left(f_{t} y_{t}+p_{t} x_{t}+a_{t} A_{t}+h_{t} s_{t}-\phi v_{t} d_{t}-\phi p_{t}^{b} q_{t}\right) & \\
s . t . & x_{t}+s_{t-1}=d_{t}+s_{t}+q_{t} & \forall t=1, \ldots, T \\
& x_{t}=A_{t} V_{t} & \forall t=1, \ldots, T \\
& q_{t} \in \mathbb{R}_{+} & \forall t=1, \ldots, T
\end{array}
$$

The term $-\sum_{t=1}^{T} \phi v_{t} d_{t}$ is a data-dependent constant and can be dropped from the objective function (4.37). Constraints (4.38) provide balance for inventory flow in which we introduce the salvage quantity $q_{t}$. Constraints (4.39) ensures replenishment of product units in only full batches.

We note that if we have $\max _{i=1, \ldots, T}\left\{\phi p_{i}^{b}\right\} \leq p_{t}, \forall t \in\{1, \ldots, T\}$, then the LSP-B-RSC is equivalent to the LSP-BD-R 2 (see Chapter 3, Section 3.4) with time dependent batch size $V_{t}$, $e_{t}=0$ (null disposed quantities), $\rho=1$ (maximum return percentage) and $j=T$ (time limit of returns).

\subsection{Conclusion}

We briefly studied the lot sizing problem under different types of capacity reservation contract (CRC) by considering the single-item case where the procurement cost has a full truckload cost structure. We started to address the general contract in different cases of capacity. This one being NP-hard in the general case, we proposed pseudo-polynomial time dynamic programming algorithms by adapting algorithms developed in the literature. After that, we incorporated different formats of CRC (the specialized and general contracts) into the lot sizing problem knowing that we gave the general form of each one. Some of them (LSP-B with deductible 
reservation, and take-or-pay contract) are shown to be equivalent to the LSP-B-CRC, which allows us to use the same methods already proposed for this latter. The others (LSP-B with payto-delay, quantity flexibility, and backup agreement contracts) have several additional restrictions compared with the LSP-B-CRC. We show that the LSP-B with revenue-sharing contract can be considered as LSP-B with buyback contract, but with some additional assumptions. 


\section{Conclusion and perspectives}

In this thesis, we have studied several lot sizing problems with batch ordering (LSP-B) under different types of capacity reservation contract: buyback, deductible reservation, take-or-pay, pay-to-delay, quantity flexibility, backup agreement, minimum commitment, and revenue-sharing contracts. Such problems draw significant attention in inventory management because they optimize the procurement activity over $T$ periods with a flexible contract between a supplier and a retailer. We have used several operational research tools: mixed integer linear program (MILP), dynamic programming algorithms, and computational experiments performed in Java and Xpress Mosel.

The first particularity of this dissertation is that we have considered the buyback contract by integrating it in the lot sizing problems under different return forms and policies. We considered three forms: $\mathrm{R}_{1}$ with $N$ fixed return periods, $w_{1}, w_{2}, \ldots, w_{N}, \mathrm{R}_{2}$ with time limit on returns, and $R_{3}$ with return possibility permitted only in procurement periods. For each form, we have two return policies of the maximum return percentage $\rho: \rho=1$ (full return) and $\rho<1$ (partial return). Concerning the form $\mathrm{R}_{1}$, we have considered three return policies of fixed return periods: acyclic $w_{i}$ with $i=1, \ldots, N\left(w_{1}<w_{2}<\cdots<w_{N} \leq T\right)$ and cyclic $w_{i}$ such that $w_{i}=i w$ with either $w=1$ or $w>1$.

The second particularity is to address the lot sizing problem with both batch ordering (FTL or OFB cost structure) and lost sales concept (non-increasing or general lost sales costs). The third particularity is to study the only full batch ordering with the disposal concept. In Table 5.1 we recapitulate the resolution methods proposed for each problem studied under the buyback contract, with the associated assumptions, as well as the computational complexity of the related algorithms. For all problems, all costs are time-dependent.

For $L S P-B R_{1}$ with $\rho=1$, we have developed four polynomial time algorithms of complexity $O(T)$ for the policy $w=1$ in order to solve the single period procurement planning with timedependent batch sizes. The replenishment is done depending on the nature of the cost structure (OFB or FTL) and on the lost sales consideration (without or with lost sales). The key idea of these algorithms is to divide the problem into $T$ independent subproblems.

Second, when the return policy is $w>1$, we have proposed four algorithms that solve the problem with constant batch size using different replenishment strategies. Two algorithms are developed for the case without lost sales, one for OFB which runs in $O(T w)$ time, and another for FTL cost structure running in $O\left(T w^{3}\right)$ time. Two other algorithms are proposed for the case with non-increasing lost sales costs whose complexities are $O\left(T w^{3}\right)$ time. The general idea of these algorithms is to divide the entire problem into $\frac{T}{w}$ independent subproblems, to solve efficiently each one and to sum up their individual costs to obtain the total replenishment cost. The algorithm for the $L S P-B_{O F B} R_{1}$ with $\rho=1$ and $w>1$ is close to the one proposed by Li et al. (2004). For the $L S P-B_{F T L} R_{1}$ with $\rho=1$ and $w>1$, we have adapted the algorithm given in Akbalik and Rapine (2018) to each subproblem. For the problems with lost sales, the idea is to use a decomposition scheme of subproblems into subplans. It will be interesting to study, in 
Table 5.1: Summary table of proposed methods for each problem under buyback contract

[1]: Lagrangian heuristic for RCSPP of Beasley and Christofides (1989); NI: Non-increasing; NN: Non-negative

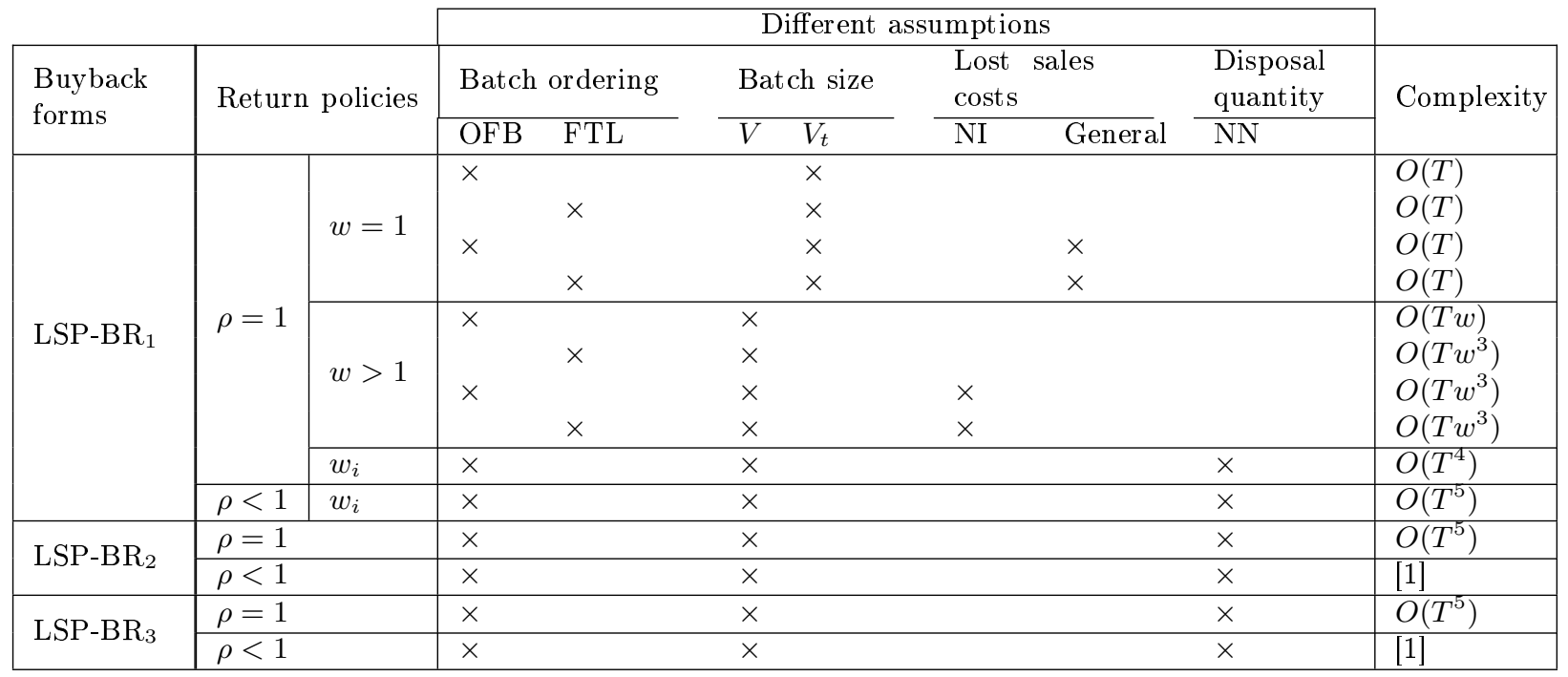

the future, $L S P-B R_{1}$ with $\rho=1$ and $w>1$ under general lost sales costs.

Third, concerning the return policy with acyclic $w_{i}$, we have studied the $L S P-B R_{1}$ with $\rho=1$ under OFB cost structure, constant batch size and disposal with null costs. The algorithm that solves this problem runs in $O\left(T^{4}\right)$ time for the general case and in $O\left(T^{2}\right)$ time for the special case of holding costs. The procedure of the latter is equivalent to the one of the $O(T w)$ time algorithm (see the 5 th line in Table 5.1).

For the $L S P-B R_{1}$ with $\rho<1$ and acyclic $w_{i}$, we have developed an algorithm in time complexity $O\left(T^{5}\right)$ lowered to $O\left(T^{4}\right)$ if a specific assumption on the return revenue and holding costs is satisfied.

For the $L S P-B R_{2}$ and $L S P-B R_{3}$ with $\rho=1$ and acyclic $w_{i}$, we have proposed two different algorithms whose running times are $O\left(T^{5}\right)$. However, with the return policy $\rho<1$, we have proved that $L S P-B R_{2}$ and $L S P-B R_{3}$ are similar to the resource constrained shortest path problem with equality and inequality resource constraints (with lower and upper resource limits) and they can be solved by the heuristic method proposed by Beasley and Christofides (1989).

An extension of these problems with non-negative and non-positive disposal costs can be easily solved by making some modifications on the solution methods proposed, in Chapter 3, to the problems with null disposal costs. An interesting extension of buyback contract to explore as perspective, is the LSP-B with another form of buyback contract, in which there are periodic limitations of the returned quantity, being a maximum return percentage of the quantity purchased: $q_{t} \leq \rho x_{t}, \forall t=1, \ldots, T$.

Each polynomial time dynamic programming (DP) algorithm proposed in this thesis has been implemented to validate its optimality and to compare its resolution time to the one of the MILP. It is fast for small and medium instances. Concerning MILP, we used the aggregate formulation and we note that the execution time is fast but it remains unpredictable because MILP depends on all the problem parameters. In order to enhance the results of MILP in the future, it will be interesting to add valid inequalities to the aggregate formulation to develop strong formulations 
with the original decision variables and to provide a polyhedral description of the convex hull of the problem.

Other interesting perspectives for the buyback contract integrated into the lot sizing problem may be the multi-item and/or the multi-level cases. For instance, one can consider a system with multiple items arriving at a retailer in batches with the possibility of returns to the supplier in every period of the planning horizon. In each period, the total returned quantity of all the items may be limited by an amount specified by the supplier. For this problem, we can refer to two research axes: multi-item LSP with batch ordering and big-bucket LSP with several items sharing a common capacity in a given period. Given the increasing complexity of the related problems and the difficulty to propose efficient algorithms, one can try to develop a dynamic programming algorithm which is polynomial for a fixed number of items (Anily and Tzur, 2005), or use polyhedral approaches by proposing valid inequalities (see Doostmohammadi and Akartunali, 2018), relaxation or decomposition techniques (Fragkos et al., 2016), or heuristics (Absi et al., 2013) for a large dimension of items. Concerning the multi-level extension, one can imagine a system with serial suppliers replenishing an item to the upstream levels in some batches, with different levels of this chain having various buyback options. In this system, one can also consider intermediate demands occurring in each echelon (direct shipment to local customers), in addition to the demand of upstream levels. We can extend the work of Zhang et al. (2012) studying the multi-echelon LSP in series with intermediate demands, by adding the concepts of batch replenishment and/or return policy. One can try to see if the valid inequalities proposed by Zhang et al. (2012) can be adapted to our problem under some restrictive assumptions.

Under the general capacity reservation contract (CRC), we have addressed the procurement LSP-B with time-varying batch sizes. The fourth particularity is that we have studied this problem in three classes of capacity: fixed, variable and constant, and, variable and time-dependent. All these problems are NP-hard. The LSP-B-CRC with fixed reserved capacity is studied by Akbalik et al. (2017) who propose a pseudo-polynomial algorithm of $O\left(T\left(D_{1, T}\right)^{2}\right)$ time complexity. For the LSP-B-CRC with variable and constant reserved capacity, we have developed a pseudopolynomial algorithm of $O\left(T\left(D_{1, T}\right)^{3}\right)$ time complexity. We have proved that the LSP-B-CRC with variable and time-dependent reserved capacity is equivalent to the LSP-B with FTL cost structure. An extension for the LSP-B-CRC is to make hypotheses on the batch size, cost and capacity parameters, for example constant batch size, non-decreasing capacities and non-increasing costs, in order to find polynomial time algorithms. Lee and Li (2013) solved the problem with the previous example but with unitary batch size $(V=1)$. Another possible extension is to generalize these current models to the multi-item case as considered in van Norden and van de Velde (2005).

The fifth particularity is that we have modeled the specialized and general contracts of CRC integrated into LSP-B by MILP. We have shown that the LSP-B with deductible reservation contract, and LSP-B with take-or-pay contract are equivalent to the LSP-B-CRC. The LSP-B with pay-to-delay, or quantity flexibility, or backup agreement contract are more complicated than the LSP-B-CRC. These problems remain open. For the LSP-B with revenue-sharing contract, we have found that the latter under cost hypothesis can be viewed as LSP-B with buyback contract. Therefore, a few research directions remain to be investigated for this problem in the general case. 


\title{
French abstract of the dissertation
}

\author{
Titre de la thèse
}

Planification d'approvisionnement par batch sous contrat de réservation de capacité

\section{Introduction et description du problème}

Dans un marché caractérisé par une forte concurrence, les entreprises cherchent à mettre en place des stratégies pour améliorer continuellement leur productivité et leur gestion des coûts en répondant à des changements de plus en plus rapides de la demande. L'utilisation de contrats flexibles d'approvisionnement est une stratégie récente et puissante pour atteindre ces objectifs. Depuis 1990, le recours aux contrats de réservation de capacité (CRC) a connu une croissance rapide. Dans ces contrats, le fournisseur permet à l'acheteur, au moment de la signature du contrat, de réserver une certaine capacité à l'avance. Au moment de la commande, l'acheteur décide du nombre de produits à se faire livrer jusqu'à la capacité réservée à un coût avantageux. Cependant, s'il a besoin d'une quantité supérieure à la capacité réservée, il peut l'obtenir à un coût plus élevé. Ainsi, cela garantit une flexibilité et un partage efficace du risque entre les membres de la chaîne logistique. Il existe différents types d'accords contractuels avec réservation de capacité : les contrats buyback, de réservation déductible, take-or-pay, pay-to-delay, de flexibilité de quantité, backup, minimum commitment et de partage des revenus. Dans cette thèse, nous abordons les contrats de réservation de capacité, et en particulier le contrat buyback sous plusieurs formes et politiques de retour, associé à la gestion des stocks dans l'approvisionnement par batch.

La gestion des stocks est une stratégie importante utilisée dans différents secteurs de l'entreprise pour améliorer la disponibilité des produits et la rapidité des livraisons, ainsi que pour aboutir à des réductions significatives de coûts, qui sont des éléments indispensables à la compétitivité. Depuis des décennies, les industries cherchent à planifier simultanément ou indépendamment les activités d'approvisionnement, de production et de transport sur un horizon de planification, en équilibrant les coûts résultant du stockage des produits avec ceux de ces différentes activités. Selon le US Census Bureau (2013), la valeur des stocks représente une somme importante, soit environ 1,6 billion de dollars aux États-Unis. Par conséquent, la gestion des stocks offre un énorme potentiel aux entreprises et à l'ensemble de l'économie. L'optimisation du dimensionnement de lots est un modèle qui permet d'éviter une accumulation excessive de stocks dans les entreprises et qui consiste à déterminer les quantités optimales à commander auprès des fournisseurs (et/ou à produire et/ou à transporter au client) et à stocker par type de produit et par période afin de satisfaire la demande au moindre coût. Dans cette étude, nous nous intéressons à l'optimisation de l'approvisionnement d'un type de produit chez un détaillant sur un horizon fini. Traditionnellement, les politiques de commandes considérées dans la gestion des stocks se 
concentrent principalement sur une taille de commande continue. Toutefois, en pratique, les produits achetés sont livrés au détaillant dans des batches, palettes, conteneurs, etc. Une autre caractéristique de notre problème de dimensionnement de lots est la considération des structures de coûts d'approvisionnement plus générales : la structure Full-Truck-Load (FTL) dans laquelle les produits sont approvisionnés dans des batches complets et fractionnaires et la structure Only-Full-Batch (OFB) dans laquelle le fournisseur exige une livraison de batches uniquement complets.

Cette thèse comprend quatre chapitres. Dans le chapitre 1, nous présentons la littérature sur les contrats d'approvisionnement et les problèmes de dimensionnement de lots avec un état de l'art détaillé en positionnant notre étude au sein des travaux précédents. Dans les chapitres 2 et 3, différentes formes de contrat buyback sont étudiées avec des différents concepts.

Dans le chapitre 2, nous étudions le problème de dimensionnement de lots avec approvisionnement par batch, sous le contrat buyback périodique dans lequel toutes les unités invendues sont retournées au fournisseur à la fin de chaque $w$ périodes avec un revenu de retour par unité. $w, 2 w, \ldots, N w$ sont les différentes périodes de retour avec $N$ étant le nombre de retours. Les paramètres $w$ et $N$ sont connus dans ce type de contrat. Nous considérons deux politiques de retour : $w=1$ en supposant des tailles de batch dépendantes du temps et $w>1$ en supposant une taille de batch constante sur l'horizon de planification. Ce problème est étudié avec les coûts d'approvisionnement FTL et OFB, en plus des coûts classiques de dimensionnement de lots. En outre, le concept de ventes perdues (lost sales) peut être pris en compte dans certains cas, en considérant un coût pour chaque unité de demande non satisfaite. L'objectif général est de déterminer les décisions d'achat, de stockage et de retour pour chaque extension de ce problème. Nous avons proposé un programme linéaire mixte en nombres entiers (PLNE) pour modéliser le problème avec les hypothèses les plus générales et également des algorithmes en temps polynomiaux pour les huit extensions considérées dans ce chapitre.

Le chapitre 3 traite le problème de la planification des approvisionnements dans un cas particulier de contrats buyback, dans lequel la quantité à acheter est limitée à un multiple d'une taille de batch constante (OFB). Dans ce chapitre, nous étudions trois formes spécifiques de contrat buyback. La première forme est celle examinée au chapitre 2, mais avec des politiques de retour plus générales qui prennent en compte différentes périodicités de retour $\left(w_{1}, w_{2}, \ldots, w_{N}\right.$ sont les différentes périodes de retour et $N$ est le nombre de retours). La seconde forme permet uniquement des retours dans les $j$ premières périodes. Dans la troisième forme, les périodes de retour doivent coïncider avec les périodes de commande. Pour chaque forme de buyback, nous associons un pourcentage de retour maximum $\rho$. Dans le cas $\rho=1$, toutes les unités invendues peuvent être retournées. Dans le cas $\rho<1$, seul un nombre limité d'unités invendues peut être retourné au fournisseur. Les valeurs de $w_{i} \forall i=1, \ldots, N$ (forme 1), $\rho$ (toutes les formes) et $j$ (forme 2) sont des paramètres de ces contrats. Tous ces problèmes de dimensionnement de lots sont étudiés avec le concept de mise au rebut (disposal). En effet, dans le cas $\rho<1$, le détaillant peut être obligé de jeter les unités qui ne peuvent pas être retournées. De plus, il peut avoir des coûts d'inventaire très élevés, et c'est plus profitable pour lui de jeter des unités que de les stocker et puis les retourner. Trois PLNE ont été proposés pour chaque forme de buyback. Pour les trois formes avec $\rho=1$, et la première avec $\rho<1$, quatre algorithmes en temps polynomiaux ont été développés. Pour la deuxième et la troisième formes avec $\rho<1$, nous avons montré que nous pouvons appliquer le seul algorithme proposé pour le problème du plus court chemin avec des contraintes de ressources d'inégalité à double face (limites inférieure et supérieure).

Dans le chapitre 4, nous avons modélisé les autres types de contrat de réservation de capacité intégrés au problème de dimensionnement de lots avec l'approvisionnement par batch et proposé des algorithmes pseudo-polynomiaux pour certains problèmes, en laissant les autres comme des 
cas ouverts. Enfin, nous finalisons ce manuscrit par une conclusion et des perspectives.

\section{Etat de l'art}

Le problème étudié dans cette thèse concerne la planification de l'approvisionnement par batch sous contrat de réservation de capacité dans un système composé d'un détaillant et d'un fournisseur. Il existe plusieurs types de contrat de réservation de capacité, mais nous étudions particulièrement le contrat buyback, intégré au problème de dimensionnement de lots sous les hypothèses variées : fonction de coût linéaire par morceaux, perte de ventes, mise au rebut, périodes de retour qui peuvent être fixes ou des variables de decision, etc. Nous avons donc positionné notre étude selon deux axes de recherche : les contrats de la chaîne logistique et le problème de dimensionnement de lots (PDL).

Il existe une littérature abondante traitant des différents problèmes d'approvisionnement sous des contrats de réservation de capacité, mais nous pouvons les classer en trois types de problèmes. Premièrement, plusieurs articles se concentrent sur la détermination des paramètres optimaux de ce contrat afin de minimiser le coût total de la chaîne logistique, voir par exemple Silver et Jain (1994), Brown et Lee (1997), Jain et Sliver (1995), Wu et al. (2005). Deuxièmement, d'autres travaux s'intéressent à la conception des contrats de réservation de capacité pour assurer la coordination des canaux en augmentant les profits des deux parties. Le lecteur peut se référer à Tsay et Lovejoy (1999), Erkoc et Wu (2005), Jin et Wu (2007). Le dernier type de problème concerne la recherche sur l'optimisation de la planification d'approvisionnement sur plusieurs périodes avec la réservation de capacité qui s'est développée au cours des dernières années. Inderfurth et Kelle (2013) et Park et Kim (2014) ont étudié ce problème sous différents paramètres et ont proposé des approches permettant d'étudier les relations acheteur-fournisseur et de trouver la structure optimale de la politique d'achat.

Le travail de cette thèse concerne notamment un type particulier de contrat de réservation de capacité : le contrat buyback dans lequel l'acheteur a la possibilité de retourner un certain pourcentage des unités non utilisées au fournisseur pour un revenu inférieur ou égal au coût d'achat. Ce contrat est utilisé dans de nombreux contextes industriels, et en particulier tout produit dont la durée de vie est limitée par la dégradation physique (produits laitiers, produits de boulangerie, produits pharmaceutiques et cosmétiques, etc.), les produits présentant un risque d'obsolescence (vêtements de mode, logiciels, magazines, journaux, etc.) et des produits dont les coûts de transport sont élevés ou des produits avec des demandes rapidement saturées (livres, disques, etc.), voir Pasternack (1985) et Hou et al. (2010). Dans la littérature, le contrat buyback est principalement utilisé dans le cadre de planification sur une seule période. Selon Hou et al. (2010), il y a trois facteurs qui conduisent à utiliser ce type de contrat. Le premier représente les propriétés du produit : périssabilité (Pasternack, 1985), mono ou multi-produit (Brown et al., 2008), et attitude de risque sur les membres de la chaîne logistique (Choi et al., 2008). Le deuxième facteur est relatif aux caractéristiques de la demande : incertitude (Marvel et Peck, 1995), et dépendance des prix (Yao et al., 2008). Le troisième facteur concerne la structure de la chaîne logistique : mono ou multi-niveaux (Song et al., 2008), et type de canal (Yao et al., 2005).

Dans un grand nombre d'articles, le contrat buyback a été étudié pour sa conception et sa mise en œuvre dans différentes configurations de la chaîne logistique. À notre connaissance, aucune recherche n'a étudié le problème de la planification d'approvisionnement avec le contrat buyback.

Dans ce travail, nous intégrerons le contrat buyback avec différentes formes et politiques de 
retour entre un détaillant et un fournisseur dans un PDL à multi-périodes avec approvisionnement par batch.

Le PDL avec approvisionnement par batch a été introduit par Lippman (1969) pour un seul produit. L'auteur a proposé un algorithme exact de programmation dynamique pour des coûts nuls de setup, une taille de batch constante et des coûts d'achat, de transport et de stockage dépendants du temps. Li et al. (2004) ont ensuite proposé une amélioration de ces résultats avec des hypothèses plus générales (des coûts positifs de setup et demandes avec backlogging). Akbalik et Rapine (2018) ont également étudié ce PDL sans backlogging mais avec multi-sources d'approvisionnement (plusieurs fournisseurs).

Quelques articles traitent le PDL avec contrat de réservation de capacité : Atamturk et Hochbaum (2001), Lee et Li (2013), Zhang (2015), Phouratsamay (2017). Toutes ces études supposent des coûts unitaires dans le contrat de réservation de capacité au lieu de coûts fixes par batch. Quelques articles étudient ce dernier problème : Van Norden et van de Velde (2005) ont proposé une heuristique Lagrangienne pour le cas multi-produits, sans coûts unitaires d'achat et avec une capacité constante de réservation, et Akbalik et al. (2017) ont developpé un algorithme de programmation dynamique en temps pseudo-polynomial pour le cas à mono-produit.

Dans certains PDL étudiés dans cette thèse, on prend en compte le concept de ventes perdues (lost sales). Quelques travaux sur les PDL avec pertes de ventes sont publiés dans la littérature. Aksen et al. (2003) ont étudié ce problème dans le cas mono-produit, sans capacité de production et ont proposé un algorithme de programmation dynamique en temps $O\left(T^{2}\right)$ pour le cas avec des coûts variables dans le temps. Ce problème a été également étudié par Absi et al. (2013) pour le cas multi-produits. Les auteurs proposent une heuristique Lagrangienne pour trouver des solutions réalisables et une métaheuristique basée sur la recherche de voisinage pour améliorer ces solutions.

Nous avons également considéré le concept de mise au rebut (disposal). Dans la littérature, il existe deux grandes catégories de PDL dans lesquels l'option de mise au rebut est intégrée. La première catégorie comprend les PDL avec planification de la production et remanufacturing, voir Richter et Weber (2001), Golany et al. (2001), etc. La deuxième catégorie comprend les PDL avec des discounts sur la quantité d'achat dans lesquels le détaillant peut se trouver dans une situation où il est plus économique d'acheter une quantité importante dépassant la demande, puis de jeter tous les stocks restant avec des coût de mise au rebut positifs ou négatifs. Ce problème a reçu très peu d'attention dans la littérature. Voir Sethi (1984) pour le cas avec deux taux de remise, une demande constante, et des coûts positifs de mise au rebut, Mirmohammadi et Eshghi (2012) pour le cas avec des coûts positifs et/ou negatifs de mise au rebut, et Li et al. (2012) pour un cas plus général avec plusieurs taux de remise.

\section{Positionnement et contributions par rapport à la littérature}

Notre première contribution consiste à résoudre un PDL déterministe sous un contrat buyback qui n'a pas encore été exploré dans la littérature. Cependant, il existe quelques articles sur le PDL sous un contrat de réservation de capacité (Atamturk et Hochbaum, 2001; van Norden et van de Velde, 2005; Lee et Li, 2013; Akbalik et al., 2017; Phouratsamay, 2017).

Nous considérons les structures FTL et OFB des coûts d'approvisionnement. Ces structures sont étudiées par de nombreux auteurs dans la littérature (Li et al., 2004; Akbalik et Rapine, 2013, 2018), mais sans considérer le contrat buyback ni ventes perdues.

En outre, nous étudions trois formes de contrat buyback utilisées dans des différentes industries. La première consiste à retourner sur des périodes de retour fixes $\left(\mathrm{R}_{1}\right)$, la seconde est 
avec une limite de temps sur les retours $\left(\mathrm{R}_{2}\right)$, et dans la troisième forme, le retour est autorisé uniquement pendant la période d'approvisionnement $\left(\mathrm{R}_{3}\right)$. Pour chaque type de buyback, nous étudions deux politiques de retour en fonction de la valeur du pourcentage de retour $\rho$ : une politique de retour complet $(\rho=1)$ et une politique de retour partiel $(\rho<1)$. En ce qui concerne $\mathrm{R}_{1}$, nous considérons également trois politiques de retour : retour cyclique avec soit $w=1$, soit $w>1$ et retour acyclique avec différentes périodes de retour autorisées.

La seconde contribution consiste à ajouter le concept de mise au rebut pour éliminer les unités qui ne peuvent être ni stockées ni retournées au fournisseur. Notre problème est différent des PDL avec remanufacturing car il n'y a pas des produits retournés du client au détaillant. Nous nous sommes intéressés au contrat buyback dans lequel le détaillant renvoie les produits non vendus au fournisseur et élimine les produits restants. Ainsi, au niveau du détaillant, dans une période $t$, la quantité retournée est un flux sortant et non pas un flux entrant comme dans les PDL inversés avec remanufacturing. L'option de mise au rebut est intégrée car les produits sont commandés par batches. Notre travail ressemble au PDL avec discount sur les quantités d'achat, et reventes de Li et al. (2012), mais nous ne pouvons pas appliquer leur algorithme dont le temps d'exécution dans notre cas tend vers l'infinie dû au nombre illimité de batch à acheter.

La troisième contribution permet de résoudre le PDL à la fois avec approvisionnement par batch et avec ventes perdues. A notre connaissance, ce problème n'a jamais été abordé dans la littérature. Nos contributions dans la littérature pour le contrat buyback sont illustrées dans la figure 6.1 en couleur jaune.

- Van Norden et van de Velde (2005)

- Molina et al. (2009)

- Akbalik et al. (2017)

Notre étude

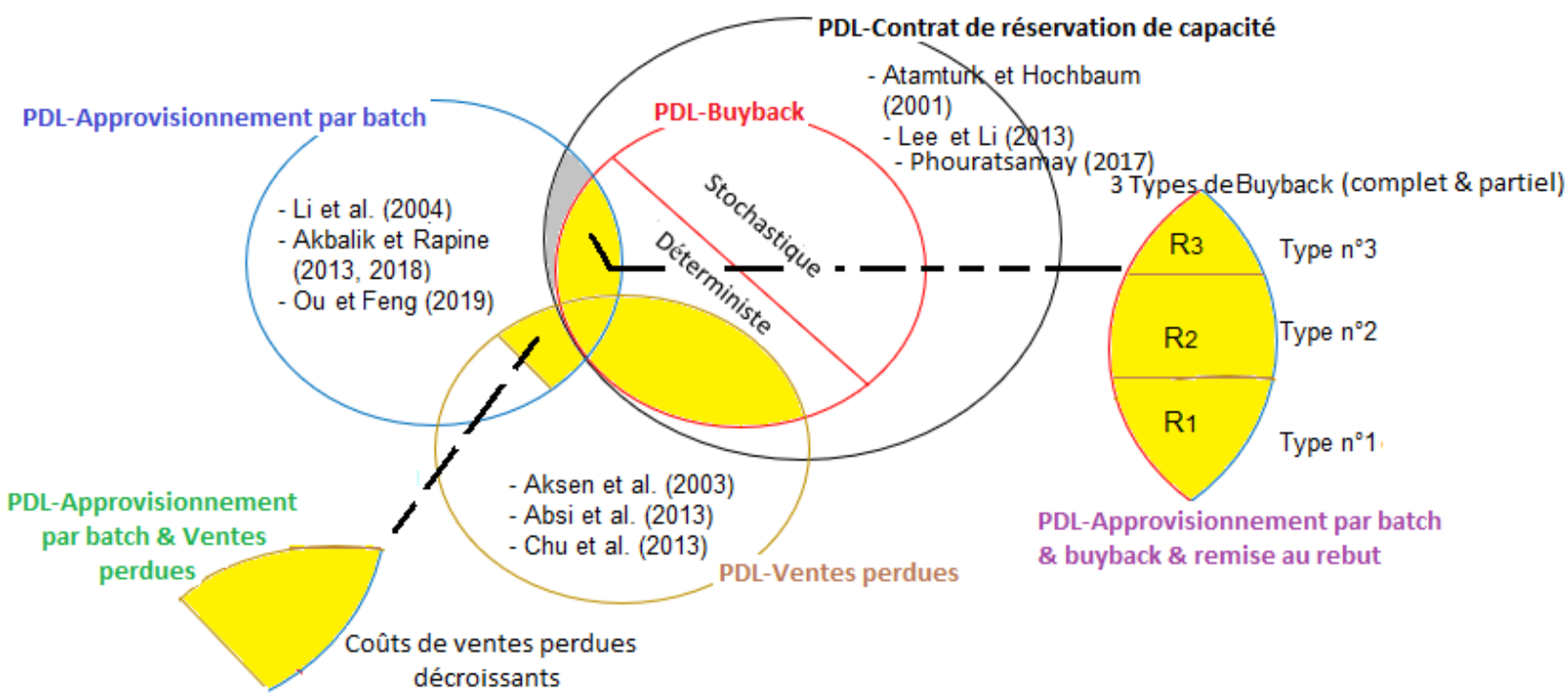

Figure 6.1: Nos contributions pour le contrat buyback

Nous étudions aussi le PDL sous un contrat de réservation de capacité. La quatrième contribution est donc d'étudier ce problème avec des tailles de batch dépendantes du temps en considérant trois classes de capacité réservée : fixe (dépendante du temps) ou variable de decision (constante ou dépendante du temps). Nous proposons des algorithmes pseudo-polynomiaux pour certains d'entre eux.

La dernière contribution consiste à modéliser les contrats spéciaux et généraux de CRC 
incorporés dans des PDL. Nous montrons que le PDL-B (B pour Batch) avec le contrat de réservation déductible, et le PDL-B avec le contrat take-or-pay sont équivalents au PDL-B avec CRC (PDL-B-CRC).

\section{Résumé des principaux résultats}

Nous donnons le tableau 6.1 qui récapitule tous les problèmes étudiés sous les différentes formes de contrat buyback, avec les hypothèses supposées, et les résultats de complexité.

Table 6.1: Tableau récapitulatif des méthodes proposées pour chaque problème étudié sous contrat buyback

[1]: Heuristique Lagrangienne de Beasley et Christofides (1989); D : Décroissants; G : Générals; P : Positive

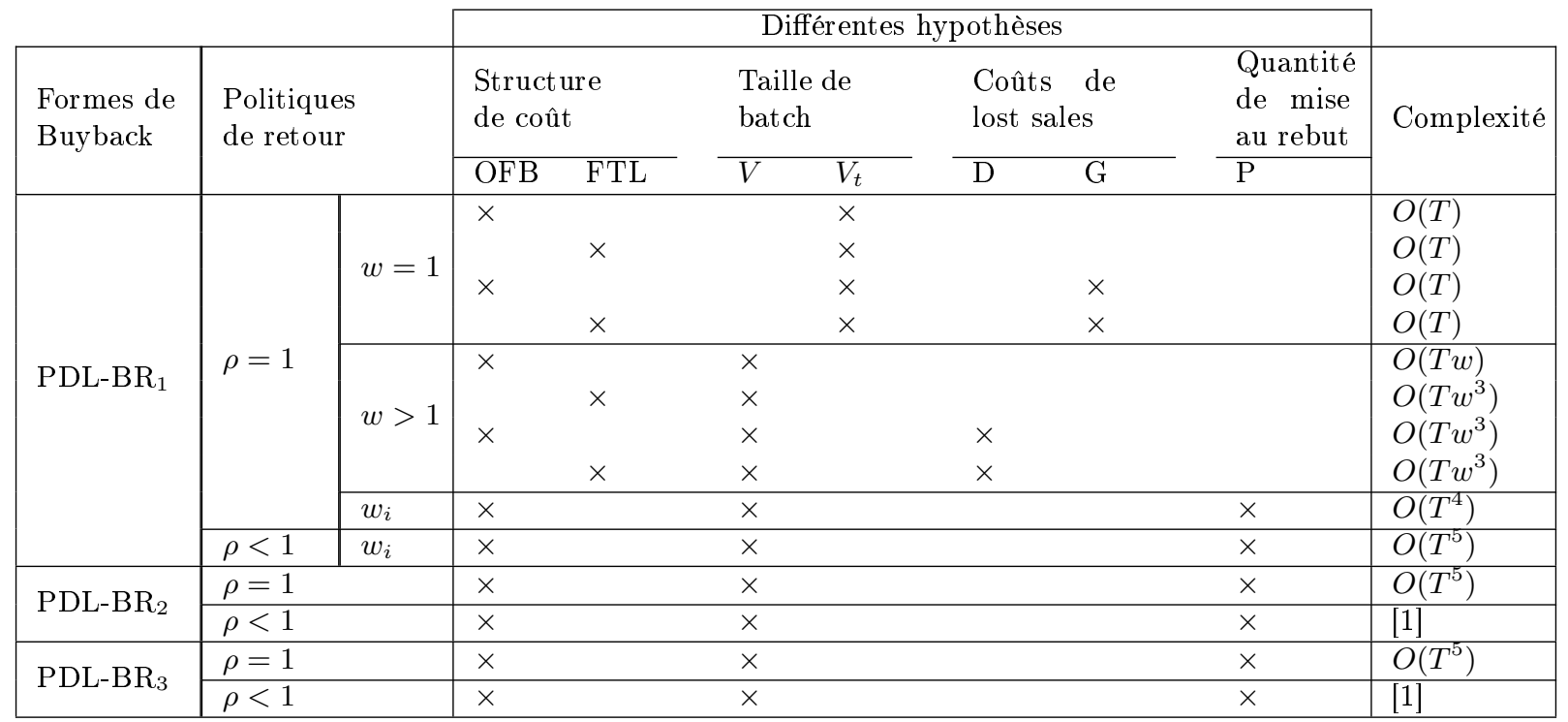

Pour résoudre le PDL-B-CRC où une capacité de réservation constante est une variable de décision, nous avons proposé un algorithme de programmation dynamique de complexité $O\left(T\left(D_{1, T}\right)^{3}\right)$ avec $D_{1, T}$ la demande cumulative de la première période 1 jusqu'à la dernière période $T$.

\section{Perspectives}

En perspectives, nous visons à étudier le $\mathrm{PDL}^{-\mathrm{BR}_{1}}$ avec $\rho=1$ et $w>1$ en considérant des coûts de ventes perdues généraux. En plus, il sera intéressant d'examiner le PDL-B avec une autre forme de contrat buyback qui impose des limitations périodiques de la quantité à retourner. D'autres perspectives intéressantes pour le contrat buyback peuvent être les problèmes avec multi-produits et/ou multi-niveaux. Par exemple, on peut envisager un système avec plusieurs produits qui arrivent dans des batches chez un détaillant avec la possibilité de retours au fournisseur à chaque période de l'horizon de planification. La quantité totale retournée à la période $t$ de tous les produits est limitée par une quantité spécifiée par le fournisseur, $\forall t=1, \ldots, T$. En ce qui concerne l'extension à multi-niveaux, on peut imaginer un système avec plusieurs fournisseurs 
en série qui réapprovisionnent un type de produit dans des batches des niveaux en amont. Ces différents niveaux disposent de divers contrats buyback.

Les PDL-B avec les contrats pay-to-delay, de flexibilité de quantité, backup, minimum commitment et de partage des revenus sont des problèmes plus complexes que PDL-B-CRC. Ces problèmes restent ouverts. 
FRENCH ABSTRACT OF THE DISSERTATION 


\section{Appendix}

\section{An aggregated formulation of the LSP-BD- $\mathrm{R}_{2}$}

To obtain the formulation without inventory variables, first, we sum up the material balance equations for the first $t$ periods for each $t \in\{1, \ldots, T\}$ and we get $s_{t}=\sum_{k=1}^{t} x_{k}-\sum_{k=1}^{t} d_{k}-$ $\sum_{k=1}^{t} q_{k}-\sum_{k=1}^{t} e_{k}$ by using $s_{0}=0$. Second, we use the constraints $s_{t} \geq 0, \forall t=1, \ldots, T$ in order to obtain the constraints without inventory variables $\sum_{k=1}^{t} x_{k} \geq \sum_{k=1}^{t}\left(d_{k}+q_{k}+e_{k}\right)$. Third, we replace $s_{t}=\sum_{k=1}^{t} x_{k}-\sum_{k=1}^{t} d_{k}-\sum_{k=1}^{t} q_{k}-\sum_{k=1}^{t} e_{k}$ in the aggregated objective function to obtain the modified objective function $\sum_{t=1}^{T}\left(f_{t} y_{t}+\tilde{p_{t}} x_{t}+a_{t} A_{t}-\tilde{p_{t}^{b}} q_{t}-\tilde{p}_{t}^{e} e_{t}-h_{t} \sum_{i=1}^{t} d_{i}\right)$.

$\min \sum_{t=1}^{T}\left(f_{t} y_{t}+p_{t} x_{t}+a_{t} A_{t}+h_{t} s_{t}-p_{t}^{b} q_{t}+0 e_{t}\right)$

Material balance equations:

$x_{t}+s_{t-1}=d_{t}+s_{t}+q_{t}+e_{t}$

$\forall t=1, \ldots, T$

$s_{0}=0$

Nonnegativity constraints of inventory variables:
$s_{t} \in \mathbb{R}_{+}$
$\forall t=1, \ldots, T$

We did not mention the other constraints because they are the same in both formulations. 


\section{Transformation of Graph $G$}

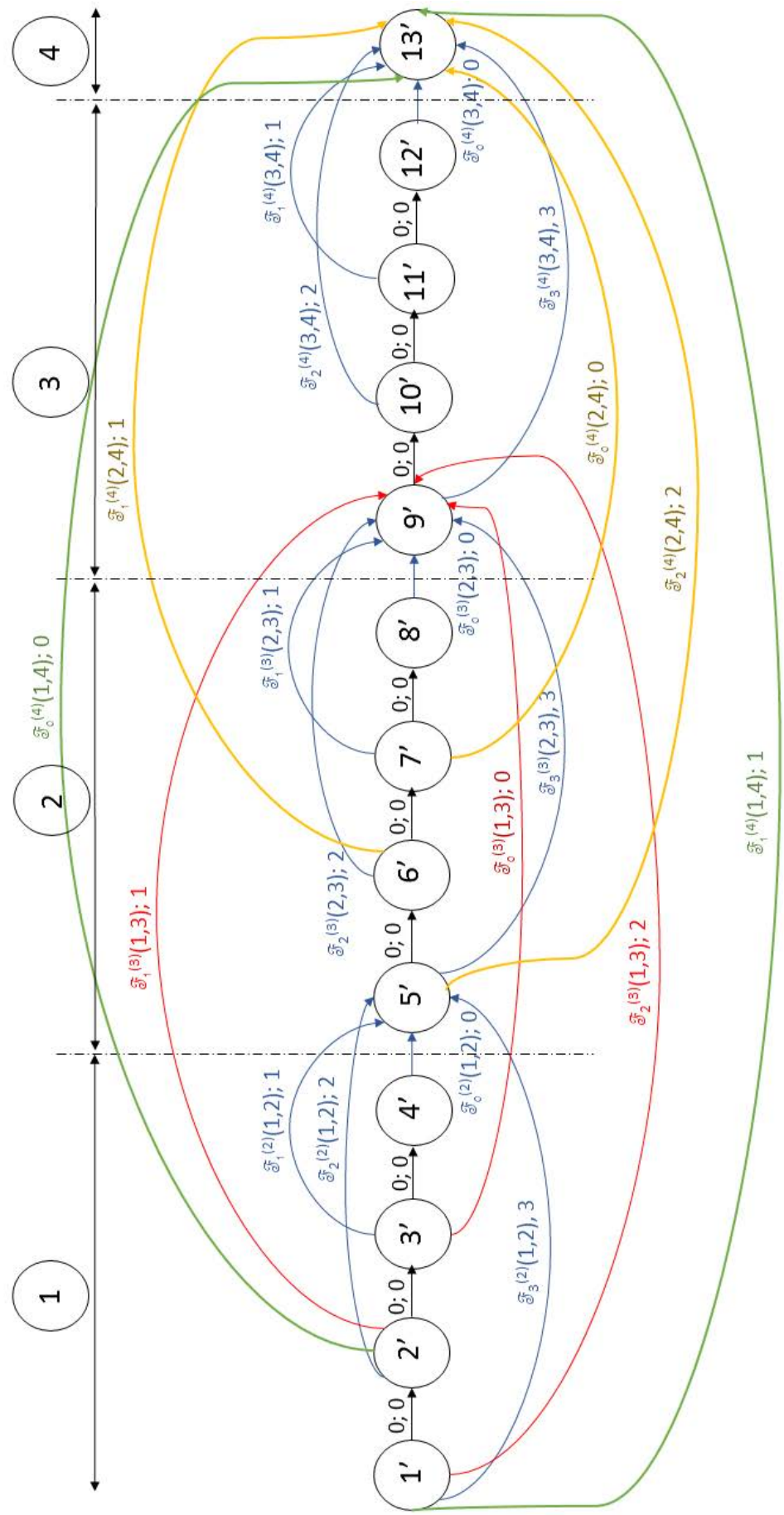

Figure 7.1: Graph $G^{\prime}$ of Example 3.4 
APPENDIX 


\section{Bibliography}

Absi, N., S. Kedad-Sidhoum, S. Dauzère-Pérès, Uncapacitated lot-sizing problem with production time windows, early productions, backlogs and lost sales, International Journal of Production Research 49(9) (2011) 2551-2566.

Absi, N., B. Detienne, S. Dauzère-Pérès, Heuristics for the multi-item capacitated lot-sizing problem with lost sales, Computers $\& 3$ Operations Research 40 (2013) 264-272.

Aggarwal, A., J.K. Park, Improved algorithms for economic lot-size problem, Operations Research 41(3) (1993) 549-571.

Aissaoui, N., M. Haouari, E. Hassini, Supplier selection and order lot sizing modeling : A review, Computers $\mathcal{G}$ Operations Research 34(25) (2007) 3516-3540.

Akbalik, A., Y. Pochet, Valid inequalities for the single-item capacitated lot sizing problem with stepwise costs, European Journal of Operational Research 198(2) (2009) 412-434.

Akbalik, A., B. Penz, Comparison of just-in-time and time window delivery policies for a single-item capacitated lot sizing problem, International Journal of Production Research 49(9) (2011) 2567-2585.

Akbalik, A., C. Rapine, Polynomial time algorithms for the constant capacitated single-item lot sizing problem with stepwise production cost, Operations Research Letters 40(5) (2012) 390397.

Akbalik, A., C. Rapine, The single item uncapacitated lot-sizing problem with time-dependent batch sizes: NP-hard and polynomial cases, European Journal of Operational Research 229(2) (2013) 353-363.

Akbalik, A., B. Penz, C. Rapine, Capacitated lot sizing problems with inventory bounds, Annals of Operations Research 229 (1) (2015) 1-18.

Akbalik, A., A. B. Hadj-Alouane, N. Sauer, H. Ghribi, NP-hard and polynomial cases for the single-item lot sizing problem with batch ordering under capacity reservation contract, European Journal of Operational Research 257(2) (2017) 483-493.

Akbalik, A., C. Rapine, Lot sizing problem with multi-mode replenishment and batch delivery, Omega 81 (2018) 123-133.

Aksen, D., K. Altinkemer, S. Chand, The single-item lot-sizing problem with immediate lost sales, European Journal of Operational Research 147(3) (2003) 558-566.

Aksen, D.,Loss of customer goodwill in the uncapacitated lot-sizing problem, Computers 86 Operations Research 34(9) (2007) 2805-2823.

Amrani-Zouggar, A., Impact des contrats d'approvisionnement sur la performance de la chaîne logistique : Modélisation et simulation, PhD thesis, Université Bordeaux (2009).

Anily, S., M. Tzur, Algorithms for the multi-item multi-vehicles dynamic lot sizing problem, Naval Research Logistics 53(2) (2006) 157-169.

Anupindi, R., R. Akella, Diversification under supply uncertainty, Management science 39(8) (1993) 915-1038. 
Anupindi, R., Y. Bassok, Supply Contracts with Quantity Commitments and Stochastic Demand, S. Tayur, R. Ganeshan and M. Magazine, eds, Quantitative Models for Supply Chain Management, Kluwer Academic Publisher, Boston (1999).

Appleinsider, Apple chip suppliers gearing up capacity for 'iPhone 7' production - report, from https://forums. appleinsider.com/discussion/191903 (2017).

Arcelus, F.J., S. Kumar, G. Srinivasan, Evaluating manufacturer's buyback policies in a single-period two-echelon framework under price-dependent stochastic demand, Omega: International Journal of Management Sciences 36 (5) (2008) 808-824.

Archetti, C., L. Bertazzi, M.G. Speranza, Polynomial Cases of the Economic Lot Sizing Problem with Cost Discounts, European Journal of Operational Research 237(2) (2014) 519-527.

Atamturk, A., D.S. Hochbaum, Capacity acquisition, subcontracting and lot-sizing, Management Science 47(8) (2001) 1081-1100.

Bassok, Y., R. Anupindi, Analysis of supply contracts with total minimum commitment, IIE Transactions 29 (1997) 373-381.

Beasley, J.E., N. Christofides, An algorithm for the resource constrained shortest path problem, Networks, 19 (1989) 379-394.

Bellman, R., Dynamic Programming, Princeton University Press, Princeton, NJ, 1957.

Biel, K., C.H. Glock, Systematic literature review of decision support models for energyefficient production planning, Computers and Industrial Engineering 101 (2016) 243-259.

Bijvank, M., I.F.A. Vis, Lost-sales inventory theory: A review, European Journal of Operational Research 215 (2011) 1-13.

Bitran, G.R., H.H. Yanasse, Computational complexity of the capacitated lot size problem, Management Science 28(10) (1982) 1174-1186.

Bose I., P. Anand, On returns policies with exogenous price, European Journal of Operational Research 178 (2007) 782-788.

Brahimi, N., Production planning: New lot-sizing models and algorithms, PhD Thesis, Université de Nantes (2004).

Brahimi, N., S. Dauzère-Pérès, N.M. Najid, A. Nordli, Single item lot sizing problems, European Journal of Operational Research 168(1) (2006) 1-16.

Brahimi, N., N. Absi, S. Dauzère-Pérès, A. Nordli, Single-item dynamic lot-sizing problems: An updated survey, European Journal of Operational Research 263(3) (2017) 838-863.

Brooke, A.F., Great expectations: Assessing the contract damages of the take-or-pay producer, Texas Law Review 70 (1992) 1469-1487.

Brown, A.O., H.L. Lee, Optimal Pay-to-Delay Capacity Reservation with Application to the Semiconductor Industry, Working Paper, Stanford University (1997).

Brown, A., M.C. Chou, C.S. Tang, The implications of pooled returns policies, International Journal of Production Economics 111 (1) (2008) 129-146.

Burnetas, A., P. Ritchken, Option contracts in supply chains, Technical report, Department of Operations, Weatherhead School of Management, Case Western Reserve Universtiy, (2002).

Buschkühl, L., F. Sahling, S. Helber, H. Tempelmeier, Dynamic capacitated lot-sizing problems:a classification and review of solution approaches, OR Spectrum 32(2) (2010) 231-261.

Cachon, G.P., Supply chain coordination with contracts, In S. Graves \& T. de Kok, eds, Handbooks in operations research and management science: Supply chain management (2002).

Cachon, G.P., M.A. Lariviere, Supply Chain Coordination with Revenue-Sharing Contracts: Strengths and Limitations, Management Science 51(1) (2005) 30-44.

Cachon, G.P, C. Terwiesch, Matching Supply with Demand: An Introduction to Operations Management. New York, McGraw-Hill International Edition (2006). 
Carlyle, W.M., J.O Royset, R.K Wood, Lagrangian relaxation and enumeration for solving constrained shortest-path problems, Networks 52(4) (2008) 256-270.

Chen, Z., and R. Zhang, Capital flow constrained lot sizing problem with loss of goodwill and loan, arXiv preprint arXiv:1708.08098 (2017).

Choi, T., D. Li, H. Yan, Mean-variance analysis of a single supplier and retailer supply chain under a returns policy, European Journal of Operational Research 184 (1) (2008) 356-376.

Chu, F., C. Chu, Polynomial algorithms for single item lot sizing models with bounded inventory and backlogging or outsourcing, IEEE Transactions on Automation Science and Engineering $4(2)$ (2007) 233-251.

Chu, C., F. Chu, J. Zhong, S. Yang, A polynomial algorithm for a lot-sizing problem with backlogging, outsourcing and limited inventory, IEEE Transactions on Automation Science and Engineering 64(1) (2013) 200-210.

Chung, C.S, C.H.M. Lin, An $O\left(T^{2}\right)$ algorithm for the NI/G/NI/ND capacitated lot sizing problem, Management Sciences 34 (1988) 420-426.

Chung, C.S., J. Flynn, C.H.M. Lin, An effective algorithm for the capacitated single item lot size problem, European Journal of Operational Research 75 (1994) 427-440.

Chung, W., S. Talluri, R. Narasimhan, Flexibility or Cost Saving? Sourcing Decisions with Two Suppliers, Decision Sciences Journal, 41 (3) (2010) 623-650.

Copil, K., M. Wörbelauer, H. Meyr, H. Tempelmeier, Simultaneous lot sizing and scheduling problems: a classification and review of models, OR Spectrum 39(1) (2017) 1-64. (2017)

Corbett, C.J., X.D Croote, A supplier's optimal quantity discount policy under asymmetric information, Management Science 46(7) (2000) 444-450.

Costa, D., E.A. Silver, Exact and approximate algorithms for the multi-period procurement problem where dedicated supplier capacity can be reserved, OR Spectrum, 18(4) (1996) 197-207.

Ding, D., J. Chen, Coordinating a three level supply chain with flexible return policies, Omega 36 (5) (2008) 865-876.

Dobos, I., A. Wimmer, Buyback and return policies for a book publishing firm, Working Paper, Vállalatgazdaságtan Intézet, Budapest, (2010).

Drexl, A., A. Kimms, Lot sizing and scheduling survey and extensions, European Journal of Operational Research 99 (1997) 221-235.

Emmons, H., S.M. Gilbert, The role of returns policies in pricing and inventory decisions for catalogue goods, Management Science 44 (1998) 276-283.

Eppen, G.D., A.V. Iyer, Backup agreements in fashion buying - the value of upstream flexibility, Management Science 43(11) (1997) 1469-1484.

Eppstein, D., Finding the k shortest paths, SIAM Journal on Computing 28 (1997) 652-673.

Erkoc, M., S.D. Wu, Managing high-tech capacity expansion via reservation contracts, Production and Operations Management 14(2) (2005) 232-251.

Farhat, M., A. Akbalik, N. Sauer, A.B. Hadj-Alouane, Problème de dimensionnement de lots sous le contrat buyback et production par batch, ROADEF Metz (2017a).

Farhat, M., A. Akbalik, N. Sauer, A.B. Hadj-Alouane, Procurement planning with batch ordering under periodic buyback contract, Congress IFAC 50(1) (2017b) 13982-13986.

Farhat, M., A. Akbalik, N. Sauer, A.B. Hadj-Alouane, Inventory replenishment planning with a full truckload cost and a full return policy, Congress INCOM 51(11) (2018a) 259-263.

Farhat, M., A. Akbalik, A.B. Hadj-Alouane, N. Sauer, Lot sizing problem with batch ordering under periodic buyback contract and lost sales, International Journal of Production Economics (2018b) (2018) 208 (2019) 500-511.

Farhat, M., A. Akbalik, N. Sauer, A.B. Hadj-Alouane, Planification d'approvisionnement sous un contrat buyback périodique avec possibilité de mise au rebut, ROADEF Lorient (2018c). 
Fatemi Ghomi, S.M.T., S.S. Hashemin, An analytical method for single level-constrained resources production problem with constant set-up cost, Journal Iranian Journal of Science and Technology - Material \& industrial engineering 26 (2002) 69-82.

Federgruen, A., M.A. Tzur, A simple forward algorithm to solve general dynamic lot sizing models with n periods in $O(n)$ or $O(n)$ time, Management Science 37(8) (1991) 909-925.

Florian, M, .K. Lenstra, A.H.G. Rinnooy Kan, Deterministic production planning: Algorithms and complexity, Management Science 26(7) (1980) 669-679.

Garcia, R., Resource constrained shortest paths and extensions, PhD thesis, Georgia Institute of Technology (2009).

Garey, M.R, D.S Johnson, Computers and intractability: a guide to the theory of NPcompleteness, New York: W.H.Freeman, (1979).

Glock, C.H, E.H. Grosse,J .M. Ries, The lot sizing problem: A tertiary study, International Journal Production Economics 155 (2014) 39-51.

Golany, B., J. Yang, G. Yu, Economic lot-sizing with remanufacturing options, IIE Transactions 33 (2001) 995-1003.

Goldberg, V.P., J.R. Erickson, Quantity and price adjustment in long-term contracts: A case study of petroleum coke, Journal of Law and Economics 30(30) (1987) 369-398.

Guan, Y., S. Ahmed, G.L. Nemhauser, A.J. Miller, A branch-and-cut algorithm for the stochastic uncapacitated lot-sizing problem, Mathematical Programming 105 (2006) 55-84.

Haase, K., Lot sizing and Scheduling for Production Planning, Springer-Verlag, (1994) New York.

Hanssmann, F., Operations Research in Production and Inventory Control, John Wiley, (1962).

Harris, F.W., How many parts to make at once, The Magazine of Management 10 (1913) $135-136$.

Hindi, K.S., K. Fleszar, C. Charalambous, An effective heuristic for the CLSP with setup times, Research Paper, Department of Systems Engineering, Brunel University, UB8 3PH, England, (2001).

van Hoesel, S., R. Kuik, M. Salomon, L.N. Van Wassenhove, The single item discrete lot-sizing and scheduling problem: Optimization by linear and dynamic programming, Discrete Applied Mathematics 48(3) (1994) 289-303.

van Hoesel, C.P.M., A.P.M. Wagelmans, $O\left(T^{3}\right)$ algorithm for the economic lot-sizing problem with constant capacities, Management Science 42(1) (1996) 142.

van Hoesel, C.P.M., A.P.M. Wagelmans, Fully polynomial approximation schemes for singleitem capacitated economic lot-sizing problems, Mathematics of Operations Research 26(2) (2001) 339-357.

Hou, J., A.Z. Zeng, L. Zhao, Coordination with a backup supplier through buy-back contract under supply disruption, Transportation Research Part E: Logistics and Transportation Review 46(6) (2010) 881-895.

Hsu, V.N, Dynamic economic lot size model with perishable inventory, Management Science 46(8) (2000) 1159-1169.

Huang, D., Z.K. Lin, W. Wei, Optimal production planning with capacity reservation and convex capacity costs, Advances in Production Engineering \&3 Management 13(1) (2018) 31-43.

Hwang, H.C., Dynamic lot-sizing model with production time windows, Naval Research Logistics 54(6) (2007) 692-701.

Hwang, H.C., Economic Lot-Sizing for Integrated Production and Transportation, Operations Research 58(2) (2010) 428-444. 
Hwang, H.C., W. van den Heuvel, A.P. Wagelmans, The economic lot-sizing problem with lost sales and bounded inventory, IIE Transactions 45 (8) (2013) 912-924.

Hwang, H.C., J. Kang, Two-phase algorithm for the lot-sizing problem with backlogging for stepwise transportation cost without speculative motives, Omega 59 (2016) 238-250.

Inderfurth, K., P. Kelle, R. Kleber, Dual sourcing using capacity reservation and spot market: Optimal procurement policy and heuristic parameter determination, European Journal of Operational Research 225 (2) (2013) 298-309.

Jain, K., E.A. Silver, The single period procurement problem where dedicated supplier capacity can be reserved, Naval Research Logistics, 42(6) (1995) 915-934.

Jans, R., Z. Degraeve, Modeling industrial lot sizing problems: a review, International Journal of Production Research 46 (6) (2008) 1619-1643.

Jaruphongsa, W., S. Çetinkaya, C.Y. Lee, Outbound shipment mode considerations for integrated inventory and delivery lot-sizing decisions, Operations Research Letters 35(6) (2007) 813-822.

Jin, M., S.D. Wu, Modeling capacity reservation in high-tech manufacturing, Working paper, Lehigh University, Pennsylvania, (2001).

Jin, M, S.D. Wu, Capacity reservation contracts for high-tech industry, European Journal of Operational Research 176 (2007) 1659-1677.

Joskow, P.L., Contract duration and relationship specific investments: Empirical evidence from coal markets, American Economic Review 77(3) (1987) 168-185.

Karimi, B., S.M.T. Fatemi Ghomi, J.M. Wilson, The capacitated lot sizing problem: a review of models and algorithms, Omega 31(5) (2003) 365-378.

Kaiser, M.J., S. Tumma, Take-or-pay contract valuation under price and private uncertainty, Applied Mathematical Modelling, 28 (2004) 653-676.

Katz, P., A. Sadrian, P. Tendick, Telephone companies analyze price quotations with Bellcore POSS software, Interfaces 24 (1994) 50-63.

Kleindorfer P.R., D.J. Wu, Integrating long and short term contracting via business-tobusiness exchanges for capital intensive industries, Management Science 49(19) (2003) 15971615.

Kleinknecht, J., R. Akella, Supply Games with Options in E-Business, INFORMS, Annual Meeting, San Jose CA, November 17-20, (2002).

Koca, E., H. Yaman, M.S. Akturk, Lot Sizing with Piecewise Concave Production Costs, INFORMS Journal on Computing 26(4) (2014) 767-779.

KRAVETZ, S., Mattel Revamps Retail Strategy To Boost Sales, Find New Avenues, Wall Street Journal, (1999).

Krishnan, H., R. Kapuscinski, D.A. Butz, Coordinating contracts for decentralized supply chains with retailer promotional effort, Management Science 50(1) (2004) 48-63.

Lariviere, M., Supply Chain Contracting and Coordination with Stochastic Demand, S. Tayur, R. Ganeshan and M. Magazine, eds, Quantitative Models for Supply Chain Management, Kluwer Academic Publisher, Boston (1999).

Lee, C.Y, A solution to the multiple set-up problem with dynamic demand, IIE Transactions 21 (1989) 266-270.

Lee, H.L., V. Padmanabhan, S. Whang, Information distortion in a supply chain : The bullwhip effect, Management Science 43(13) (1997) 546-558.

Lee, C.H., Coordinated stocking, clearance sale, and return policies for a supply chain, European Journal of Operational Research 131 (3) (2001) 491-513.

Lee, C. Y., S. Çetinkaya, W. Jaruphongsa, A dynamic model for inventory lot sizing and outbound shipment scheduling at a third-party warehouse, Operations Research 51 (5) (2003) 
$735-747$.

Lee, C.Y., X. Li, Economic lot sizing: the capacity reservation model, Operations Research Letters 41 (2013) 670-674.

Leung, J.M., T.L. Magnanti, R. Vachani, Facets and algorithms for capacitated lot sizing, Mathematical Programming 45 (1989) 331-359.

Li, C.L., V.N. Hsu, W.Q. Xiao, Dynamic Lot Sizing with Batch Ordering and Truckload Discounts, Operations Research 52(4) (2004) 639-654.

Li, C.L., J. Ou, V.N. Hsu, Dynamic lot sizing with all-units discount and resales, Naval Research Logistics 59(3-4) (2012) 230-243.

Lippman, S.A., Optimal inventory policy with multiple set-up costs, Management Science 16(1) (1969) 118-138.

Lopez, M.A., B.G. Kingsmans, The economic lot scheduling problem: Theory and practice, International Journal of Production Economics 23 (1991) 147-164.

Lorenz, D.H., D. Raz, A simple efficient approximation scheme for the restricted shortest path problem, Operations Research Letters 28 (2001), 213-219.

Luo, C., X. Tian, X. Mao, Q. Cai, Coordinating Supply Chain with Buy-Back Contracts in the Presence of Risk Aversion, Asia-Pacific Journal of Operational Research 35(2) (2018).

Manne, A.S., Programming of economic lot sizes, Management Science 4(2) (1958) 115-135.

Marple, C.J., A. Roland, How state commissions are treating take-or-pay settlement costs, Public Utilities Fortnightly 124(9) (1989) 18-22.

Marvel, H., J. Peck, Demand uncertainty and returns policies, International Economic Review 36(3) (1995) 691-714.

Mirmohammadi, H.S., K. Eshghi, A hybrid polynomial time algorithm for the dynamic quantity discount lot size model with resale, Computer Operation Research 39 (2012) 1771-1778.

Molina, F., M.O. Santos, F.M.B. Toledo, S.A. Araujo, An approach using Lagrangean/surrogate relaxation for lot-sizing with transportation costs, Pesquisa Operacional 28(2) (2009) 269-288.

Molina, F., R. Morabito R, S.A. Araujo, MIP models for production lot sizing problems with distribution costs and cargo arrangement, Journal of the Operational Research Society 67(11) (2016) 1395-1407.

Moussay, J., Les contrats-cadres d'achat $80 \%$ de nos besoins, Forum Rhodia, Juin 2002, Mesures 746.

Van Norden, L., S. Van de Velde, Multi-product lot-sizing with a transportation capacity reservation contract, European Journal of Operational Research 165 (2005) 127-138.

Okhrin, I., K. Richter, The linear dynamic lot size problem with minimum order quantity, International Journal of Production Economics 133 (2) (2011) 688-693.

Ou, J., J. Feng, Production lot-sizing with dynamic capacity adjustment, European Journal of Operational Research 272(1) (2019) 261-269.

Özdamar, L., M.A. Bozyel, The capacitated lot sizing problem with overtime decisions and setup times, IIE Transactions 32 (2000) 1043-1057.

Padmanabhan, V., I.P.L Png, Returns Policies: Make Money by Making Good, Sloan Management Review/Fall (1995) 65-72.

Padmanabhan, V., I.P.L Png, Manufacturer's Returns Policies and Retail Competition, Marketing Science 16(1) (1997) 81-94.

Pahl, J., S. Voß, Integrating deterioration and lifetime constraints in production and supply chain planning: A survey, European Journal of Operational Research 238(3) (2014) 654-674.

Papachristos, S., I. Ganas, Optimal policy and stability regions for the single product periodic review inventory problem, Journal of the Operational Research Society 49(2) (1998) 165-75. 
Park, S.I., J.S. Kim, A mathematical model for a capacity reservation contract, Applied Mathematical Modelling 38(5-6) (2014) 1866-1880.

Pasternack, B.A., Optimal pricing and returns policies for perishable commodities, Marketing Science 4(2) (1985) 166-176.

Phouratsamay, S.L, Coordination des décisions de planification dans une chaîne logistique à deux niveaux, $\mathrm{PhD}$ thesis, Université pierre et marie-curie (2017).

Piñeyro, P., O. Viera, Inventory policies for the economic lot-sizing problem with remanufacturing and final disposal options, Journal of Industrial and Management Optimization 5(2) (2009) 217-238.

Piñeyro, P., O. Viera, The economic lot-sizing problem with remanufacturing and one-way substitution, International Journal of Production Economics 124 (2010) 482-488.

Pochet, Y., L.A. Wolsey, Lot-sizing with constant batches: Formulation and valid inequalities, Mathematics of Operations Research 18(4) (1993) 767-785.

Ribeiro, C. C., M. Minoux, A heuristic approach to hard constrained shortest path problems, Discrete Applied Mathematics 10 (1985) 125-137.

Richter, K., J. Weber, The reverse Wagner/Whitin model with variable manufacturing and remanufacturing cost, International Journal of Production Economics 71 (2001) 447-456.

Sandbothe, R.A., G.L. Thompson, A forward algorithm for the capacitated lot size model with stockouts, Operations Research 38 (3) (1990) 474-486.

Schrader, C., Speeding Build and Buy Processes Across a Collaborative Manufacturing Network, ASCET Project (2001) 82-88.

Serel, D.A., M. Dada, H. Moskowitz, Sourcing decisions with capacity reservation contracts, European Journal of Operational Research 131 (2001) 635-648.

Serel, D.A., Capacity reservation under supply uncertainty, Computers 8 Operations Research 34(4) (2007) 1192-1220.

Sethi, S.P, A quantity discount lot size model with disposals, International Journal of Production Research 22(1) (1984) 31-39.

Shi, K., T Xiao, Coordination of a supply chain with a loss-averse retailer under two types of contracts, International Journal Information and Decision Sciences 1(1) (2008) 5-25.

Silver, E.A., k. Jain, Some ideas regarding reserving supplier capacity and selecting replenishment quantities in a project context, International Journal of Production Economics 35 (1994) 177-182.

Sohn, K.I, H. Hwang, A dynamic quantity discount lot size model with resales, European Journal Operational Research 28 (1987) 293-297.

Song, Y., S. Ray, S. Li, Structural properties of buyback contracts for price setting newsvendors, Manufacturing and Service Operations Management 10 (1) (2008) 1-18.

Stadtler, H., Improved rolling schedules for the dynamic single-level lot-sizing problem, Management Science 46 (2) (2000) 318-326.

Stanley E.D., D.P. Honig, L. Gainen, Linear programming in bid evaluation, Naval Research Logistics Quarterly (1954) 48-54.

Tarim, S.A, B.G. Kingsman, The stochastic dynamic production/inventory lot-sizing problem with service-level constraints, International Journal of Production Economics 88(1) (2004) 105119.

Toy, A.Ö., E. Berk, Dynamic lot sizing problem for a warm/cold process, IIE Transactions 38 (11) (2006) 1027-1044.

Tsay, A.A., W. Lovejoy, Quantity fexibility contracts and supply chain performance, Manufacturing and Service Operations Management 1(23) (1999) 89-111. 
Tsay, A.A., The quantity flexibility contract and supplier-customer incentives, Management Science 45(10) (1999) 1339-1358.

Turner, L., Variants of the Shortest Path Problem, Algorithmic Operations Research 6 (2011) 91-104.

Van Vyve, M., Algorithms for single-item lot-sizing problems with constant batch size, Mathematics of Operations Research 32 (3) (2007) 594-613.

Wagelmans, A., S. Van Hoesel, A. Kolen, Economic lot sizing: An $\mathrm{O}(\mathrm{nlog}(\mathrm{n}))$ algorithm that runs in linear time in the wagner whitin case, Operations Research 40(1) (1992) 145-156.

Wagner, H.M., T.M. Whitin, Dynamic version of the economic lot size model, Management Science 5(1) (1958) 89-96.

Wang, Y., P. Zipkin, Agents'incentives under buy-back contracts in a two-stage supply chain, International Journal Production Economics 120 (2009) 525-539.

Wolsey, L. A., Uncapacitated lot-sizing problems with start-up costs, Operations Research 37 (5) (1989) 686-848.

Wu, S.D., M. Erkoc, S. Karabuk, Managing capacity in the high-tech industry: a review of literature, The Engineering Economist 50 (2005) 125-158.

$\mathrm{Wu}, \mathrm{D}$., Coordination of competing supply chains with news-vendor and buyback contract, International Journal of Production Economics 144(1) (2013) 1-13.

Xiong, H., C. Bintong, J. Xie, A composite contract based on buy back and quantity flexibility contracts, European Journal of Operational Research 210 (2011) 559-67.

Yao, M.J., S.E. Elmaghraby, The economic lot scheduling problem under power-of-two policy, Computers and Mathematics with Applications 41 (2001) 1379-1393.

Yao, D., X. Yue, X. Wang, J.J. Liu, The impact of information sharing on a returns policy with the addition of a direct channel, International Journal of Production Economics 97 (2) (2005) 196-209.

Yao, Z., S.C.H. Leung, K.K. Lai, Analysis of the impact of price-sensitivity factors on the returns policy in coordinating supply chain, European Journal of Operational Research 187 (1) (2008) 275-282.

Yazlali, O., F. Erhun, Managing demand uncertainty with dual supply contracts on capacity and inventory: A heuristic approach, Stanford University working paper, (2006).

Zangwill, W. I., A backlogging model and a multi-echelon model of a dynamic economic lot size production systema network approach, Management Science 15 (9) (1969) 506.

Zhang, M., S., Kucukyavuz, H. Yaman, A polyhedral study of multi-echelon lot sizing with intermediate demands, Operations Research 60(4) (2012) 918-935.

Zhang, M., Capacitated lot-sizing problem with outsourcing, Operations Research Letters 43(5) (2015) 479-483.

Zhu, X., The dynamic resource-constrained shortest path problem on an acyclic graph with application in column generation and a literature review on sequence-dependent scheduling, $\mathrm{PhD}$ thesis, Texas A\&M University (2005).

Zhu, X., W.E. Wilhelm, Three-stage approaches for optimizing some variations of the resource constrained shortest-path sub-problem in a column generation context, European Journal of Operational Research 183 (2007) 564-577.

Zhu, X., W.E. Wilhelm, A three-stage approach for the resource-constrained shortest path as a sub-problem in column generation, Computers 83 Operations Research 39 (2012) 164-178.

Ziegelmann, M., Constrained shortest paths and related problems, PhD thesis, Saarland University (2001). 
BIBLIOGRAPHY 


\title{
Résumé
}

Nous nous intéressons au Problème de Dimensionnement de Lots mono-produit (PDL) dans une chaîne logistique composée d'un détaillant et d'un fournisseur en y intégrant le contrat buyback et l'approvisionnement par batch. L'objectif est de déterminer un plan d'approvisionnement pour le détaillant pour satisfaire ses demandes déterministes sur un horizon fini, tout en minimisant ses coûts d'approvisionnement et de stockage. Concernant le coût d'approvisionnement, nous supposons deux structures différentes : FTL (Full Truck Load) et OFB (Only Full Batch). Trois types de contrat buyback sont étudiés : avec des périodes de retour fixes, avec une limite de temps sur les retours, et avec des retours uniquement dans les périodes d'approvisionnement. Chaque contrat est caractérisé par un pourcentage de retour maximal qui peut être égal à 100\% (retour total) ou inférieur à 100\% (retour partiel). Pour le PDL sous le contrat buyback avec des périodes de retour fixes, nous supposons le cas de ventes perdues (lost sales). En outre, un autre concept ajouté dans les PDL sous les trois types de contrat buyback réside dans le fait que le détaillant peut jeter la quantité invendue et non retournée au fournisseur, appelé mise au rebut (disposal). Nous avons modélisé ces différentes extensions du PDL par des Programmes Linéaires en Nombres Entiers (PLNE). Nous avons ensuite développé des algorithmes exacts polynomiaux de programmation dynamique pour certaines extensions, et montré la NP-difficulté pour d'autres. Pour chaque problème résolu en temps polynomial, nous avons comparé l'efficacité et les limites de l'algorithme proposé avec celles des quatre formulations en PLNE. Nous avons également proposé des modèles mathématiques pour les PDL sous d'autres types de contrats de réservation de capacité dans le cas déterministe à multi-périodes.

Mots-clés: Problème de dimensionnement de lot, approvisionnement en batch, contrat buyback, ventes perdues, mise au rebut, PLNE, algorithme polynomial, complexité

\begin{abstract}
We study the single-item Lot Sizing Problem (LSP) in a supply chain composed of a retailer and a supplier by integrating the buyback contract and the batch ordering. The purpose is to determine a replenishment planning for the retailer to satisfy his deterministic demands over a finite horizon, while minimizing the procurement and inventory costs. Regarding the procurement cost, we assume two different structures: FTL (Full Truck Load) and OFB (Only Full Batch). We consider three types of buyback contract: with fixed return periods, with a time limit on returns, and with returns permitted only in procurement periods. Each contract is characterized by the maximum return percentage being either equal to $100 \%$ (full return) or less than $100 \%$ (partial return). For the LSP under the buyback contract with fixed return periods, we assume the concept of lost sales. Another concept considered in the LSP's under the three types of buyback contract is the disposal of the unsold and unreturned quantities. We model these different LSP extensions as a Mixed Integer Linear Program (MILP). Thereafter, we develop exact polynomial time dynamic programming algorithms for some extensions and show the NP-hardness of others. For each problem solved in polynomial time, we compare the efficiency and the limits of the proposed algorithm with those of four MILP formulations by performing different tests. Finally, we propose mathematical models for the LSP's under other types of the capacity reservation contract in the deterministic and multi-period case.
\end{abstract}

Keywords: Lot sizing problem, batch ordering, buyback contract, lost sales, disposal, MILP, polynomial time algorithm, complexity 
